RALF SCHNELL (HRSG.)

\title{
Wahrnehmung Kognition Ästhetik
}

Neurobiologie und

Medienwissenschaften 
Ralf Schnell (Hrsg.)

Wahrnehmung - Kognition - Ästhetik 
Die Reihe »Medienumbrüche« wird herausgegeben von Ralf Schnell. 
Ralf Schnell (Hrsg.)

Wahrnehmung - Kognition - Ästhetik

Neurobiologie und Medienwissenschaften

[transcript $] \quad$ Medienumbrüche I Band 12 
Diese Arbeit ist im Kulturwissenschaftlichen Forschungskolleg 615 der Universität Siegen entstanden und wurde auf seine Veranlassung unter Verwendung der von der Deutschen Forschungsgemeinschaft zur Verfügung gestellten Mittel gedruckt.

\section{Bibliografische Information der Deutschen Bibliothek}

Die Deutsche Bibliothek verzeichnet diese Publikation in der Deutschen Nationalbibliografie; detaillierte bibliografische Daten sind im Internet unter http://dnb.ddb.de abrufbar.

\section{(C) 2005 transcript Verlag, Bielefeld}

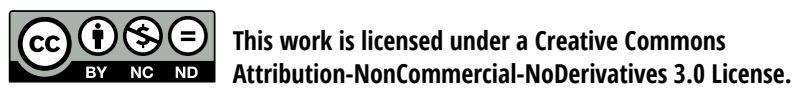

Umschlaggestaltung: Susanne Pütz, Siegen;

Kordula Röckenhaus, Bielefeld

Druck: Majuskel Medienproduktion GmbH, Wetzlar

ISBN 3-89942-347-X

Gedruckt auf alterungsbeständigem Papier mit chlorfrei gebleichtem Zellstoff.

Besuchen Sie uns im Internet: http://www.transcript-verlag.de

Bitte fordern Sie unser Gesamtverzeichnis und andere Broschüren an unter:info@transcript-verlag.de 


\section{INHAL T}

Ralf Schnell

Medienwissenschaft und Neurobiologie. Zur Einführung in diesen Band .... 7

Gerhard Roth

Wahrnehmung: Abbildung oder Konstruktion?......................................... 15

Eckart Voland

Das ,Handicap-Prinzip“ und die biologische Evolution

der ästhetischen Urteilskraft.

Manfred Fahle

Ästhetik als Teilaspekt bei der Synthese menschlicher Wahrnehmung. 61

Siegfried Frey

Das Bild vom Andern:

Funktionsprinzipien der visuellen Eindrucksbildung.

Eckart Altenmüller

Musik - die Sprache der Gefühle? Neurobiologische Grundlagen

emotionaler Musikwahrnehmung

Karl Clausberg

„Ein Mikroskop für die Zeit“. Was Benjamin und Klages,

Einstein und das Kino den fernen Sternen verdanken

K. Ludwig Pfeiffer

Ich lese, also bin ich (nicht)

Peter M. Hejl

Medienwissenschaften und Wahrnehmungsbiologie.

Zum Problem einer Nicht-Beziehung

Autorenverzeichnis 

RALF SCHNELL

\title{
MEDIENWISSENSCHAFT UND NEUROBIOLOGIE.
}

\section{ZUR EINFÜHRUNG IN DIESEN BAND}

\begin{abstract}
Was haben die Schädelnerven der Wirbeltiere mit Dichtung zu tun? Was sucht die vergleichende Anatomie im Monolog des dramatischen Helden? Welcher Weg führt von der Kiemenhöhle der Fische zur menschlichen Komödie, von rhythmisierter Prosa zur Ausstellung des Gehirns in den Gesichtsnerv?
\end{abstract}

So fragt im Jahr 1995 ein junger Lyriker namens Durs Grünbein anlässlich seiner Danksagung zur Verleihung des renommiertesten deutschen Literaturpreises. ${ }^{1}$ Seine Fragen nennt er selber „seltsame Fragen“, denn, so Grünbein unter ausdrücklichem Hinweis auf den Namenspatron jenes Preises: ,sie allein zeigen an, wohin es führen muß, wenn Literatur sich auf das Reale einläßt, wenn den Stil das Naturstudium prägte, der zoologische Fakt und das ärztliche Gutachten Einzug hielten in Novelle und Drama... bis das Genre gesprengt lag, Fragmente die Folge, fieberhafte Notate, somatische Poesie“.2.

Derjenige, dem Grünbein zutraute, jene ,seltsamen Fragen“ beantworten zu können, war freilich „,tot, jung gestorben an Typhus, infiziert, wie man annimmt, beim Aufschneiden von Fischpräparaten ${ }^{\text {‘3 }}$ : Georg Büchner nämlich, Autor der Dramen Dantons Tod und Woyzeck, des Lustspiels Leonce und Lena, Verfasser der Erzählung Lenz und Herausgeber des revolutionären Hessischen Landboten. In seiner naturwissenschaftlichen Dissertation mit dem Titel Sur le système nerveux de barbeaux hatte Büchner jene „vollständige Transformation“ vorbereitet, die

1 Grünbein, Durs: Den Körper zerbrechen. Rede zur Verleihung des GeorgBüchner-Preises 1995, gehalten am 21. Oktober 1995 im Staatsheater Darmstadt; Frankfurt a.M. 1995, S. 7.

2 Grünbein (wie Anm. 1).

3 Grünbein (wie Anm. 1), S. 7f. 
Grünbein „Physiologie aufgegangen in Dichtung “4 ${ }^{4}$ nennt. Nach Annahme seiner Arbeit als Dissertationsleistung, 1836, mit 23 Jahren, ein halbes Jahr vor seinem Tod, hält der Dichter und Revolutionär in Zürich seine Probevorlesung mit dem Titel Über Schädelnerven. In dieser Vorlesung unterscheidet Büchner den „teleologischen“ Standpunkt in den Naturwissenschaften, der alle physiologischen Entwicklungen unter den Aspekten Funktionalität und Zweckmäßigkeit wahrnimmt, vom ,philosophischen“ Standpunkt, für den die Natur ,in allen ihren Äußerungen sich unmittelbar selbst genug“ ist: „Alles, was ist, ist um seiner selbst willen da. “5 Doch ist es, nach Überzeugung Büchners, weder der philosophischen noch der teleologischen „Methode“, weder „der Anschauung des Mystikers“ noch „dem Dogmatismus der Vernunftphilosophen“ gelungen, eine „Brücke“ zu schlagen zum „Naturleben, das wir unmittelbar wahrnehmen".6 Deshalb setzt sich der junge Wissenschaftler nichts Geringeres zum Ziel, als - in kenntnisreicher Auseinandersetzung mit den fortgeschrittensten naturhistorischen Studien seiner Zeit (Oken, Carus, Arnold) - seinerseits „dem Naturstudium eine andere Gestalt zu geben“". ${ }^{7}$ Er geht, um über Genese und Funktion der Schädelnerven tragfähige Aussagen machen zu können, zurück auf die ,einfachsten Formen“ der Evolutionsgeschichte, in der Gewißheit: „Die einfachsten Formen leiten immer am sichersten, [weil in] ihnen sich nur das Ursprüngliche, absolut Notwendige zeigt. “8 ${ }^{8}$ Diese Gewissheit regt seinen Scharfblick wie seinen Scharfsinn an, stimuliert seine Beobachtungsgabe, führt zu überlegten Vergleichen und ermöglicht prägnante Urteile - eine anregende, wenn nicht aufregende Lektüre.

Georg Büchners Zürcher Antrittsvorlesung ist in jeder Hinsicht lehrreich. Doch nicht, dass alle Einsichten Büchners gegenwärtig, im Licht fortgeschrittener naturwissenschaftlicher Erkenntnis, Bestand hätten, macht ihren Wert aus, sondern dass von seiner Methodik des Forschens sich immer noch lernen lässt. Georg Büchner arbeitet, so würden wir heute sagen, ,transdisziplinär'. Ihn interessiert das Philosophische in der Anatomie, das materiell Physiologische in der Geschichte, das Verhältnis von Körperlichkeit und Erkenntnisvermögen, von Physis und Aisthesis. Büchner wirft mithin in seiner Antrittsvorlesung höchst aktuelle Fragen auf: Fragen nach dem Zusammenspiel von Gehirn und

4 Grünbein (wie Anm. 1), S. 8f.

5 Büchner, Georg: „Über Schädelnerven“, in: Werke und Briefe. Gesamtausgabe, hrsg. v. Fritz Bergemann, Wiesbaden 1958, S. 350.

6 Büchner (wie Anm. 5), S. 351.

7 Büchner (wie Anm. 5).

8 Büchner (wie Anm. 5), S. 355. 
Geist, nach den physiologischen Bedingungen unseres Wahrnehmungsapparates und den reziproken Wirkungen unserer produktiven Verarbeitungsformen von Wahrnehmungs- und Imaginationsimpulsen auf unsere neurobiologische Ausstattung. Es sind Fragen, die gerade Dichter immer wieder fasziniert haben und auf die sie bisweilen faszinierende, nicht selten rätselhafte, häufig unausdeutbare Antworten gefunden haben. So, beispielsweise, Gottfried Benn in jener Prosaskizze mit dem Titel Gehirne (1915) und ihrem Thema Schizophrenie, die man, wie manch anderen der naturwissenschaftlich und medizinisch imprägnierten poetischen Texte Benns auch, als eine Art Relaisstation zu Durs Grünbein bezeichnen könnte, zu seinem Gedicht „Zerebralis“ beispielsweise, das sich im Gedichtband Schädelbasislektion (1991) findet:

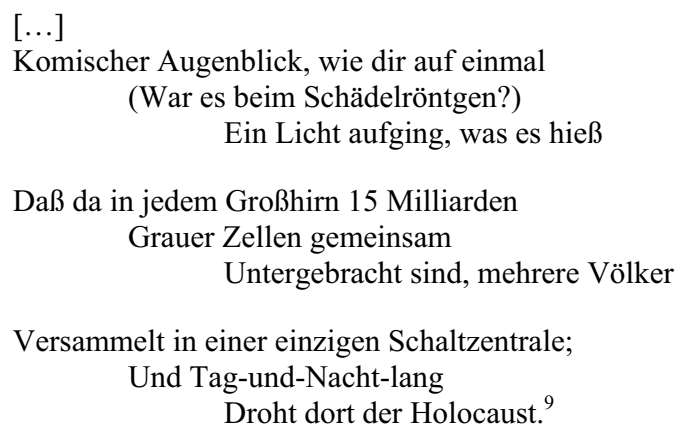

Es muss angesichts dieser - hier nur eben angedeuteten poetischen Traditionslinie - verwundern, dass man dem Zusammenspiel von „Wahrnehmung - Kognition - Ästhetik“ unter dem gemeinsamen Begriffsregister einer ,Aisthetik ${ }^{\text {- }}$ im Sinn einer Wissenschaft der physiologischen wie der ästhetisch produktiven Vermögen des Menschen - bislang noch kaum nachgegangen ist. Verwundern auch deshalb, weil zu den Axiomen der Medientheorie und Medienästhetik seit Jahrzehnten Walter Benjamins These zählt, innerhalb „großer geschichtlicher Zeiträume“ verändere sich „mit der gesamten Daseinsweise der menschlichen Kollektiva auch die Art und Weise ihrer Sinneswahrnehmung“. ${ }^{10}$ Wenn die Art und Weise, in der die menschliche Sinneswahrnehmung sich organisiert, „nicht nur natürlich, sondern auch geschichtlich bedingt“ ist - wie Ben-

9 Grünbein, Durs: Schädelbasislektion. Gedichte, Frankfurt a.M. 1991, S. 135.

10 Benjamin, Walter: „Das Kunstwerk im Zeitalter seiner technischen Reproduzierbarkeit" (Zweite Fassung), in: Gesammelte Schriften, hrsg. v. Rolf Tiedemann/Hermann Schweppenhäuser, Bd. I (2), Frankfurt a.M. 1991, S. 478 . 
jamin in seinem kanonischen Aufsatz „Das Kunstwerk im Zeitalter seiner technischen Reproduzierbarkeit" schreibt -, dann stellen sich die Fragen nach dem Zusammenhang von geschichtlicher und natürlicher Wahrnehmungsveränderung desto nachdrücklicher.

Wie also lernen wir sehen? Und wie verändern sich die erlernten Sehweisen mit den wechselnden Einflüssen, denen Menschen im Verlauf ihres Lebensprozesses ausgesetzt sind? Wie verarbeiten wir Wahrnehmungsprozesse? Was bedeuten Veränderungen im historischen Maßstab der menschlichen Wahrnehmungsgeschichte? Und welche Wirkungen sind dabei der Geschichte der Medien zuzuschreiben? In welchem Verhältnis stehen der menschliche Wahrnehmungsapparat und die Umbrüche in der Medienentwicklung zueinander? Und welche Rolle spielen in diesem Verhältnis die künstlerischen Avantgarden, insbesondere die der Mediengeschichte?

Auf solche Fragen gibt es noch kaum zureichende Antworten. Die Wissenschaften, die mit diesem Problemzusammenhang sachlich und fachlich befasst sein müssten, nämlich Medienwissenschaft und Neurobiologie, sind bislang miteinander nicht ernsthaft ins Gespräch gekommen. Diese Tatsache erscheint auch deshalb überraschend, weil sich die Neurobiologie seit einigen Jahren, ebenso unverhofft wie unübersehbar, eines großen öffentlichen Interesses erfreut. Es hat ihr in jüngster Zeit Resonanz selbst im gehobenen Feuilleton gesichert, ihr Zustimmung gebracht - mehr freilich noch Kritik eingetragen, weil sich die Debatte allzu rasch in den Begriff der „Willensfreiheit“ verbissen hatte ${ }^{11}-$, und allemal hätte diese Konjunktur die Medienwissenschaften anregen können, sich der neurobiologischen Grundlagen ihrer eigenen Theoreme allererst zu vergewissern. Umgekehrt scheint auch der Gedanke nicht abwegig, dass sich das mittlerweile hoch elaborierte Instrumentarium der Neurowissenschaften auf Phänomene der Medienentwicklung - auf Wahrnehmungsprozesse ebenso wie auf historische Zäsuren der Mediengeschichte, auf die visuelle Aufbereitung von Datenmengen generell wie auf medienästhetisch induzierte Rezeptionsprozesse im besonderen - mit der Aussicht auf einen Zugewinn an Erkenntnis applizieren ließe.

Es trifft sich deshalb gut, dass zumindest ein Gesprächsangebot der Neurowissenschaften inzwischen vorliegt. Anlass zur Ausarbeitung dieser selbstbewusst „Das Manifest“ genannten Gesprächsplattform ${ }^{12}$ bildet

11 Vgl. hierzu Geyer, Christian (Hrsg.): Hirnforschung und Willensfreiheit. Zur Deutung der neuesten Experimente, Frankfurt a.M. 2004.

12 „Das Manifest. Elf führende Neurowissenschaftler über Gegenwart und Zukunft der Hirnforschung“, in: Gehirn\&Geist, 6 (2004), S. 30-37. 
der rapide Erkenntnisfortschritt, der sich in den vergangenen beiden Jahrzehnten innerhalb dieses Forschungsfeldes vollzogen hat, vorangetrieben insbesondere durch die Entwicklungen der modernen Informationstechnologien: die digitalen Bildgebungsverfahren, die durch Hochleistungsrechner ermöglichten detailreichen Modellierungen physikochemischer Vorgänge, die Codierung, Speicherung und Selektierung hunderttausender von Neuronenschaltkreisen, deren Auswertung, so die Autoren des „Manifests“, die „,vielleicht wichtigste Erkenntnis der modernen Neurowissenschaften“" ermöglicht hat:

Geist und Bewußtsein - wie einzigartig sie auch von uns empfunden werden - fügen sich [...] in das Naturgeschehen ein und übersteigen es nicht. Und: Geist und Bewußtsein sind nicht vom Himmel gefallen, sondern haben sich in der Evolution der Nervensysteme allmählich herausgebildet. ${ }^{13}$

Die produktiven Potentiale dieser Erkenntnis übersetzt das „Manifest“ in einen programmatischen Aufruf zu einem kooperativen Austausch zwischen Disziplinen, die einander notorisch fremd gegenüber stehen: „Geisteswissenschaften und Neurowissenschaften werden in einen intensiven Dialog treten müssen, um ein gemeinsames Menschenbild zu entwerfen." ${ }^{\text {"14 }}$

Der vorliegende Band dokumentiert die konkrete Aufnahme eines solchen Dialogs. Dieser wurde - in Form einer Jahrestagung des von der Deutschen Forschungsgemeinschaft (DFG) geförderten Kulturwissenschaftlichen Forschungskollegs „Medienumbrüche“ an der Universität Siegen - bereits im November 2003, ein Jahr vor Erscheinen jenes „Manifests“, geplant und realisiert. Die für die Tagung gewählte Thematik „Wahrnehmung - Kognition - Ästhetik“ ging von einer spezifischen Voraussetzung aus, wie sie auch das „Manifest“ nahelegt: der nämlich, dass menschliche Natur und Kultur in einem koevolutiven Verhältnis zueinander stehen. Die Fragestellung impliziert mithin, dass Kulturprodukte und die Kriterien dafür, dass wir etwas ,interessant“ oder gar „schön“ finden, eine neurobiologische Grundlage besitzen, die Veränderungsimpulsen unterliegt. Jedes Lebewesen wählt aus dem, was prinzipiell wahrnehmbar ist, Objekte aus, die sich durch Relevanz auszeichnen. Die Ausbildung von Relevanzkriterien dient insoweit zunächst der Erfüllung basaler biologischer Funktionen.

13 (wie Anm. 12), S. 33.

14 (wie Anm. 12), S. 37. 
Ob diese stabil sind oder ob sie kulturell überformt oder gar transformiert werden können, bildete die zentrale medienanthropologische Fragestellung der Tagung. Wahrnehmung, Kognition und Ästhetik lassen sich in diesem Kontext als differente Verarbeitungs- und Ausdrucksformen einer biologischen Basisausstattung des Menschen verstehen, auf die sie ihrerseits - wie auch immer vermittelt - Einfluss nehmen. Zu prüfen ist, welcher Art die wechselseitigen Impulse sind, die zu kulturellen und biologischen Veränderungen führen können, welche Wirkung sie besitzen und welche Dauer ihnen zukommt. Diese Fragen richten sich nicht allein auf Prozesse wahrnehmungstechnischer, kognitiver oder ästhetischer Habitualisierungen, sondern thematisieren zugleich deren physiologische Voraussetzungen.

Das Kulturwissenschaftliche Forschungskolleg „Medienumbrüche“ hat versucht, mit dieser Tagung wissenschaftliches Neuland im Grenzgebiet der Natur- und Kulturwissenschaften zu erschließen. Die Beteiligung ausgewiesener Forscher aus den Bereichen Biologie, Medizin und Hirnforschung bot die Möglichkeit, die Ergiebigkeit der Thematik in einer Weise zu prüfen, die bewusst - durchaus orientiert am Vorbild des Dichters und Naturwissenschaftlers Georg Büchner - über die Grenzen und Schranken der Fachdisziplinen hinausführt.

Das Spektrum der Beiträge in dem hier vorliegenden Band reicht aus der Sicht der Neurowissenschaften von erkenntnistheoretischen Erwägungen, wie sie Gerhard Roth an den Anfang seines Beitrags „Wahrnehmung: Abbildung oder Konstruktion?"“ stellt, über evolutionsbiologische Voraussetzungen des ästhetischen Urteilsvermögens (Eckart Voland) und die Synthetisierungsfunktion der Ästhetik im Wahrnehmungsprozess (Manfred Fahle) bis zu den Ressourcen der visuellen Eindrucksbildung (Siegfried Frey), den neurobiologischen und anthropologischen Grundlagen der Musikwahrnehmung (Eckart Altenmüller) und dem komplexen, widerspruchsvollen Zusammenspiel von technischem Fortschritt und ästhetischer Phantasie (Karl Clausberg). Bei diesem Gespräch mit den Neurowissenschaften haben sich die Medienwissenschaftler dies sei sogleich eingeräumt - einstweilen vor allem mit der Rolle von Fragestellern beschieden. Die Beiträge von K. Ludwig Pfeiffer und Peter M. Hejl gehen gleichwohl auf Bedingungen und Voraussetzungen von Wahrnehmungen und kognitiven Prozessen ein und damit auf den neurowissenschaftlichen Rahmen ästhetischer Produktivität und Rezeptivität wie auf die epistemologischen Veränderungsimpulse, die von den Anregungen für einen langfristigen Austausch zwischen den Neurowissenschaften und den Medienwissenschaften ausgehen könnten. 
Mit der Publikation der Beiträge zu dieser Tagung verbindet sich die Hoffnung, langfristig zu einem solchen Austausch beizutragen, zumindest aber - in einem ganz pragmatischen Sinn - das wechselseitige Interesse an den jeweiligen fachspezifischen Fragestellungen in einer Weise zu wecken, die eine Fortsetzung des Austauschs für beide Seiten ertragreich erscheinen lässt.

Gerhard Roth hat bei der Vorbereitung der Tagung mit fachlichem Rat und persönlichen Empfehlungen geholfen. Dafür schuldet ihm das Kulturwissenschaftliche Forschungskolleg „Medienumbrüche“ großen Dank, ebenso unserem Kollegen Peter M. Hejl, der zu der Tagung angeregt und ihre Durchführung mitverantwortet hat. 



\section{GERHARD ROTH}

\section{WAHRNEHMUNG：ABBILDUNG ODER KONSTRUKTION? ${ }^{1}$}

Wie hängen die Inhalte unserer Wahrnehmungen mit den tatsächlichen Geschehnissen in der Welt zusammen? Welchen Wahrheits- und Realitätsgehalt haben also unsere Wahrnehmungen? Über diese erkenntnistheoretische Fragen wird nachgedacht, seit es Philosophie gibt. Die Antworten sind - wie könnte es anders sein - sehr verschieden. Die einen Erkenntnistheoretiker nennt man Realisten, weil sie davon ausgehen, dass die Inhalte unserer Wahrnehmung die Welt mehr oder weniger den „realen“ Tatsachen entsprechend wiedergeben. Andere nennt man Idealisten, weil für sie die Wahrnehmungsinhalte im Wesentlichen „ideale“ Erfindungen unseres Geistes sind und keinen ursächlichen Bezug zur Realität haben. Die Wahrheitsfrage stellt sich für einen Idealisten demnach überhaupt nicht.

Es gibt natürlich alle erdenklichen Zwischenstufen, die man kritischen Realismus, hypothetischen Realismus, radikalen oder realistischen Konstruktivismus nennt, je nachdem wie sehr deren Vertreter an Wahrnehmungsinhalte als „objektive Tatsachen“ glauben, bei der unsere Geistestätigkeit nur eine geringe Rolle spielt, oder wie sehr sie davon überzeugt sind, dass unsere Vorerfahrungen, Vorstellungen und Erwartungen und Gedanken einen erheblichen Einfluss auf unsere Wahrnehmungen haben. Schließlich gibt es noch die Skeptiker, die meinen, die Frage, wie Welt und Wahrnehmung zusammenhängen, sei unsinnig, denn die darauf gegebenen Antworten könnten gar nicht objektiv überprüft werden.

Nehmen wir unsere Alltagserfahrung, dann sind solche gedanklichen Bemühungen um den Realitätsgehalt unserer Wahrnehmungen nicht sofort einsichtig. Die Dinge und Geschehnisse meiner Welt liegen ja unmittelbar vor mir, ich sehe, höre, rieche, schmecke und ertaste sie; sie werden von mir so wahrgenommen, wie sie sind. Die Kaffeetasse mit

1 Dieser Beitrag ist eine überarbeitete Fassung eines Kapitels aus Roth, Gerhard: Aus Sicht des Gehirns, Frankfurt a.M. 2003. 
dem Zwiebelmuster vor mir ist eine Kaffeetasse mit Zwiebelmuster, was soll sie sonst sein?

\section{Farbwahrnehmung - ein komplizierter Vorgang}

Mit dem interessanten und komplizierten Problem der Farben haben sich Wahrnehmungsforscher und Erkenntnistheoretiker seit langem beschäftigt. Bei Tageslicht sieht unsere Welt bunt aus, während diese Farbenpracht umso mehr verschwindet, je dunkler es wird. Über diese Tatsache denken wir normalerweise nicht nach, obwohl wir nicht selten mit der Schwierigkeit konfrontiert werden, bei Dunkelheit Farben zu erkennen. Niemand wird aber annehmen, dass bei Dunkelheit die Dinge tatsächlich ihre Farben verlieren, vielmehr gehen wir davon aus, dass der Verlust der Farbigkeit der Welt bei Dunkelheit mit Eigenschaften unseres Sehapparats zusammenhängt.

Die Erklärung hierfür besteht darin, dass es in der Netzhaut unseres Auges zwei unterschiedliche Typen von lichtempfindlichen Sinneszellen (Photorezeptoren) gibt, nämlich Zapfen, die farbempfindlich sind, und Stäbchen, die nur Graustufen vermitteln. Die Zapfen arbeiten nur bei Tageslicht und Dämmerlicht etwa bis zu einer Helligkeit einer Vollmondnacht, während die Stäbchen bei Tageslicht ebenso wie bei Dämmerlicht aktiv sind bis hinunter zu einer Helligkeit, die weit unter der einer sternklaren Nacht liegt. Allerdings müssen sie dabei lange Gelegenheit gehabt haben, sich an die Dunkelheit anzupassen.

Das sichtbare Licht umfasst elektromagnetische Wellen mit Wellenlängen zwischen rund 400 und 700 Nanometern (d.h. Millionstel eines Millimeters). Licht nahe der unteren Grenze nennt man entsprechend „kurzwelliges Licht“, und Licht nahe der oberen Grenze „langwelliges Licht". Ersteres erscheint uns blauviolett, letzteres rot. An das kurzwellige Licht schließt sich das ultraviolette Licht an, das für unser menschliches Auge unsichtbar ist, von dem wir aber einen Sonnenbrand kriegen, und an das langwellige Licht die infrarote Strahlung, die wir als Wärme empfinden. Unsere Netzhaut besitzt drei Zapfentypen, nämlich einen für kurzwelliges Licht, einen für mittlere Wellenlängen und einen für langwelliges Licht. Allerdings liegt der Empfindlichkeitsbereich der beiden letzteren Rezeptoren beim Menschen sehr eng beieinander.

Man könnte nun meinen, Farbensehen beruhe darauf, dass bei blauem Licht der erste Zapfentypen erregt wird, bei grünem Licht der zweite und bei rotem Licht der dritte. Man spricht schließlich von Blau-, Grün- und Rotrezeptoren. Dass die Sache nicht so einfach ist, kann man 
daran sehen, dass man mit einem einzigen Zapfentyp von Photorezeptoren gar keine Farben wahrnehmen kann. Hätten wir nur den kurzwelligen Zapfentyp, so würden wir die Welt nicht etwa ganz blau, sondern ,unbunt" bzw. grau in grau sehen, so als ob wir nur Stäbchen hätten. Es müssen mindestens zwei Zapfentypen vorhanden sein, denn es kommt beim Wahrnehmen einer bestimmten Farbe nicht bloß auf die absolute Erregung der Rezeptoren an, sondern auf das jeweilige Verhältnis der Erregung verschiedener Zapfentypen an. Das menschliche Auge verfügt, wie gesagt, über drei Zapfentypen. Diese überlappen in ihrem Empfindlichkeitsbereich beträchtlich. Licht einer bestimmten Wellenlänge erregt entsprechend meist alle drei Zapfentypen, wenngleich einen Typ mehr als die anderen, und es ist das entstehende Mischungsverhältnis der Erregungen von mindestens zwei Zapfentypen, das die wahrgenommene Farbe festlegt, und nicht die absolute Erregung eines Zapfentyps.

Die Sache ist aber noch komplizierter. Unsere Welt sieht für uns vom Tagesanbruch bis zur Dämmerung hinsichtlich der Farbe der Gegenstände relativ gleich aus: Bilder, Äpfel und Autos ändern sich nicht merklich in ihrer Farbe. Eigentlich sollten sie das aber, denn wenn wir physikalische Messungen des Tageslichts machen, so stellen wir fest, dass sich der Anteil lang-, mittel- und kurzwelligen Lichtes, seine spektrale Zusammensetzung, im Tagesverlauf stark ändert. Morgens und abends dominiert in aller Regel das langwellige Licht, weil der mittelund kurzwellige Anteil des Lichtes der tiefstehenden Sonne durch die Luftschichten stärker gestreut wird als mittags. Deshalb müssten auch die Farben der Gegenstände, die das Sonnenlicht reflektieren, sich im Tagesverlauf stark ändern, was sie aber nicht tun.

Wie dieses Phänomen der Farbkonstanz zustande kommt, ist nicht ganz geklärt. Ein plausibler Erklärungsversuch geht davon aus, dass wir beim Sehen zum einen direkt das Sonnenlicht in seiner aktuellen spektralen Zusammensetzung wahrnehmen, die sich im Laufe des Tages ändert, und zum anderen das von Oberflächen reflektierte Licht, bei dem einen Teil der Wellenlängen in Abhängigkeit von der physikalisch-chemischen Beschaffenheit der Oberfläche verschluckt wird. Dieses reflektierte Licht verändert sich natürlich in dem Maße, in dem sich das Tageslichtspektrum ändert, das auf die Oberfläche fällt. Diese Veränderung kann nun unser Farbwahrnehmungssystem „herausrechnen“, da es das jeweilige Spektrum des Sonnenlichts kennt. So scheinen die Oberflächen von Objekten immer dasselbe Wellenlängenspektrum zu reflektieren und farblich gleich zu bleiben, was sie überhaupt nicht sind. Der Vorgang ähnelt dem, was die Arbeitsmarktstatistiker eine „Saisonbereinigung“ der 
Arbeitslosenzahlen nennen, denn hier werden die jahreszeitlich bedingten Schwankungen der Arbeitslosenzahl ebenfalls herausgerechnet.

Es gibt also gar keinen direkten Zusammenhang zwischen der Wellenlänge des Lichtes, das von Gegenständen reflektiert wird und auf unsere Netzhaut fällt, und einer bestimmten Farbempfindung. Dennoch sind die Beziehung zwischen Welt und Wahrnehmung keineswegs zufällig, sondern zumindest bei einfacheren Sinneswahrnehmungen gesetzmäßig, wenngleich kompliziert. Schwierig wird die Sache aber dadurch, dass wir gelegentlich Farben auch dann wahrnehmen, wenn es sie physikalisch gar nicht geben dürfte. Wenn wir etwa eine Fläche mit einer bestimmten intensiven Farbe, z.B. blau, für ca. 1 Minute anstarren und dann unseren Blick auf eine weiße Fläche lenken, so sehen wir für einige Zeit dort die Umrisse der Fläche abgebildet, das so genannte Nachbild, das allerdings in der "Gegenfarbe“ erscheint, in unserem Beispiel gelb. Bei einem grünen Gegenstand erscheint entsprechend ein rotes Nachbild, bei einer roten Fläche ein grünes Nachbild und bei einer gelben Fläche ein blaues Nachbild. Ist die Fläche dunkel, so erscheint auf einem dunklen Hintergrund ein helles Nachbild, und umgekehrt.

Dieses erstaunliche Phänomen hängt mit der Tatsache zusammen, dass bei unserer Farbwahrnehmung zwei Prinzipien miteinander verbunden sind, nämlich zum einen die Existenz von drei Zapfentypen für die geschilderten drei Wellenlängenbereiche, und zum anderen das Gegenfarbenprinzip, das darauf beruht, dass sich jeweils zwei Farbwahrnehmungsbereiche, nämlich gelb und blau sowie grün und rot, und darüber hinaus die beiden Graustufen hell und dunkel, gegenseitig „bekämpfen“, sich antagonistisch zueinander verhalten. Entsprechend spricht man auch vom Farbantagonismus.

Eine mögliche Erklärung für das Gegenfarbenprinzip lautet, dass das für blau zuständige Wahrnehmungssystem durch das längere Starren auf eine blaue Fläche „ermüdet" und dass beim anschließenden Schauen auf eine weiße Fläche das für die Gegenfarbe, nämlich gelb, zuständige System, das beim Anblick von blau unterdrückt war, eine Zeitlang die Oberhand gewinnt. Übrigens kann man im Bereich der Bewegungswahrnehmung etwas ganz Ähnliches beobachten. Starren wir einige Zeit lang ein Muster an, das sich immer in eine Richtung bewegt (z.B. ein Band mit Querstreifen), und schauen wir dann auf eine weiße Fläche, dann scheint sich dort etwas Streifenartiges in Gegenrichtung zu bewegen. Auch dies wird mit der Ermüdung von „antagonistischen“ Wahrnehmungsprinzipien, hier für die Bewegungsrichtung, erklärt. 
Unser visuelles System konstruiert in beiden Fällen etwas, das gar nicht vorhanden ist. Deshalb können Farb- und Bewegungswahrnehmung wie viele andere Wahrnehmungsinhalte keine Abbilder realer Gegebenheiten sein. Immerhin - so wird der erkenntnistheoretische Realist sagen - gibt es gesetzmäßige Beziehungen zwischen dem externen Phänomen Licht und seinen Wellenlängen und der subjektiven Wahrnehmung, auch wenn diese kompliziert sind. Aufgrund dieser gesetzmäßigen Beziehungen ist es uns möglich, den Effekt der Gegenfarb- und Gegenbewegungstäuschung vorherzusagen. Die Gegenfarben und Gegenbewegungen gibt es ja nur, weil zuvor das Auge mit einer bestimmten Farbe oder Bewegung gereizt wurde. Gäbe es keine externe Welt mit bestimmten Eigenschaften - so sagt uns der Realist -, dann könnte es auch keine solchen Täuschungen geben. Dasselbe gilt natürlich auch für die vielen optischen Täuschen, z.B. die Pfeiltäuschung (Müller-Lyer-Täuschung genannt) oder die Schienentäuschung (Ponzo-Täuschung genannt). Interessanterweise verschwindet der Täuschungseffekt hier nicht, auch wenn wir den visuellen Eindruck als Täuschung erkannt haben. Wir können die Täuschungsfigur sogar selbst malen und unterliegen ihr. Bei der Pfeiltäuschung sind die parallelen Linien objektiv gleichlang, aber irgendetwas in unseren Sinnesorganen oder unserem Gehirn behauptet, sie seien dies nicht, und da nützt auch alle Einsprache unseres Verstandes nichts.

\section{Wahrnehmung als aktiver Prozess}

Man könnte viele weitere Beispiele nennen, aus denen klar wird, dass Wahrnehmung ein aktiver Prozess ist und keine bloße Widerspiegelung der Dinge. Das dürfte auch der Realist zugeben, wenn er ein kritischer und kein naiver Realist ist, denn es geht den Tieren und uns Menschen bei der Wahrnehmung ja nicht darum, die Welt so zu erfassen, wie sie tatsächlich ist. Das wäre erstens völlig unmöglich, denn nur ein kleiner Teil dessen, was in der Welt passiert, kann überhaupt unsere Sinnesorgane erregen, und zweitens wäre es auch völlig unnütz, denn nur weniges in der Welt ist für uns von Bedeutung. Die Sinnesorgane beschränken unsere Wahrnehmung schon durch ihre Bau- und Funktionsweise auf einen sehr kleinen Ausschnitt des Gesamtgeschehens in der Welt. Dieser ist allerdings meist derjenige, der von besonderer Bedeutung für unser Überleben ist und gleichzeitig der Bereich, in dem die Sinnesorgane am besten arbeiten. Das sollte uns nicht überraschen, denn die Strukturen der Welt, der Arbeitsbereich der Sinnesorgane und der Bereich der für unser Überleben wichtigen Dinge haben sich im Laufe der Evolution einander 
angepasst - zumindest so gut, wie es eben ging. Hierauf hat die so genannte Evolutionäre Erkenntnistheorie immer völlig zu Recht hingewiesen.

Dies erklärt, warum Lebewesen in unterschiedlichen Umwelten zum Teil ganz unterschiedliche Sinnesorgane oder zumindest Sinnesorgane mit ganz unterschiedlichen Arbeitsbereichen entwickelt haben. Man denke nur an die Ultraschallortung der Fledermäuse und Delfine, die Infrarotortung der Grubenottern, Wärmerezeptoren bei Brandkäfern, den Geruchssinn der Hunde, den Magnetsinn der Vögel und so weiter. Auch nach Anschauung eines kritischen Realisten arbeiten die Wahrnehmungssysteme eindeutig selektiv, d.h. die unwichtigen Dinge werden weggefiltert und die wichtigen verstärkt, aber es bleibt ein ,realistischer“ Kernbestand in unserer Wahrnehmung, ohne den die sensorischen Anpassungsleistungen gar nicht erklärlich wären.

Wahrnehmung beruht also nicht auf einer einfachen Abbildung der Welt, einer bloßen Kopie, aber doch auf einer systematischen, wenngleich ausschnitthaften, hervorgehobenen und abgeschwächten Repräsentation der Welt im Gehirn, die mit der spezifischen Überlebenssituation des Organismus eng zusammenhängt. Wie könnte der Organismus auch überleben, wenn er nicht das Wesentliche seiner Umwelt erfasste?

Es ist nicht verwunderlich, dass unter Philosophien ebenso wie unter Wissenschaftlern der kritische Realismus der am weitesten verbreitete erkenntnistheoretische Standpunkt ist. Allerdings stellt die Aussage des kritischen Realisten, der Organismus erfasse in seiner Wahrnehmung vornehmlich dasjenige, was für sein Überleben notwendig ist, einen logischen Zirkelschluss dar. Wir stellen fest, dass die heute lebenden Organismen im Großen und Ganzen gut überleben. Daraus schließen wir, dass ihre Wahrnehmung dasjenige erfasst, was diesem Überleben dient. Dies drehen wir nun um und konstatieren, dass der Organismus nur deshalb gut überlebt, weil sein Wahrnehmungs- und Erkenntnisapparat das für das Überleben Wesentliche erfasst. Dies ist die Grundbehauptung der bereits genannten Evolutionären Erkenntnistheorie, die eine kritisch-realistische Erkenntnistheorie ist.

Diesen Zirkelschluss könnten wir am saubersten auflösen, wenn wir in der Lage wären, die Objekte und Ereignisse der Welt - bildlich gesprochen - in der einen Hand zu halten und unsere Wahrnehmungsleistungen in der anderen und beide dann zu vergleichen. Dann würden wir sehen, in welcher Beziehung sie zueinander stehen, d.h. ob unsere Wahrnehmung tatsächlich die Welt im Wesentlichen richtig wiedergibt (wenngleich ausschnittweise und über- bzw. unterbetont), oder ob es gar 
keine direkte Beziehung zwischen ihnen gibt, wie die erkenntnistheoretischen Idealisten behaupten. Letzteres würde allerdings die Rolle, welche die Wahrnehmung bei der Sicherstellung des Überlebens spielt, ziemlich rätselhaft erscheinen lassen.

Ein solcher direkter Vergleich ist aber nicht möglich, denn er verlangte die paradoxe Fähigkeit, die Welt unabhängig von unserer Wahrnehmung wahrzunehmen. Unsere Wahrnehmungswelt ist die einzige sinnliche Welt, die wir haben; die von unserer Wahrnehmung vermutete unabhängige Welt ist nicht „dahinter“, sie existiert erlebnismäßig überhaupt nicht, auch wenn wir mit gutem Grund annehmen, dass sie irgendwie vorhanden ist. Dies nennt man den erkenntnistheoretischen Zirkel; er verhindert, dass wir die Beziehung zwischen Welt und Wahrnehmung in ihrer tatsächlichen Beschaffenheit, ihrem Wahrheitsgehalt, überhaupt feststellen können. Ein solcher Zirkel entsteht immer dann, wenn Leistungen, die jemand vollbringt, nicht von irgendeinem Außenstehenden beurteilt werden, sondern von demjenigen, der sie vollbringt. Dies ist der Fall, wenn man den Schüler seine eigenen Schulleistungen beurteilen oder einen Beamten selbst darüber entscheiden ließe, ob er aufgrund seiner Leistungen befördert werden soll. Wir tun gut daran, so etwas zu unterbinden, denn eine solche Eigenbeurteilung geht immer schief. Das aber ist genau das Dilemma der Erkenntnistheorie: Mithilfe unserer Wahrnehmung und unseres Denkens sollen wir den Wahrheits- und Realitätsgehalt unserer Wahrnehmung und unseres Denkens überprüfen.

Es gibt jedoch einen gewissen Ausweg aus diesem Dilemma, besser gesagt einen Umweg, den wir bereits zu Beginn beschritten haben, nämlich im Zusammenhang mit der Farbwahrnehmung. Wir können uns nämlich mithilfe sinnesphysiologischer Methoden in begrenztem Umfang von den Fesseln unserer unmittelbaren Wahrnehmung und deren Täuschbarkeit befreien, indem wir feststellen, dass die von uns wahrgenommenen Farben gar nicht in der physikalischen Welt existieren, sondern dass es Unterschiede im Wellenlängenspektrum des sichtbaren Lichtes sind, die auf komplizierte Weise in unserem Gehirn unsere Farbwahrnehmungen bedingen. In ähnlicher Weise können wir feststellen, dass es objektiv keine Töne und Geräusche gibt, sondern unterschiedliche Schwingungen von Luftmolekülen, die wir als Schalldruckwellen bezeichnen und die auf ebenso komplizierte Weise Töne, Geräusche, Melodien und Worte in unserem Gehirn entstehen lassen.

Natürlich ist uns dabei klar, dass die Forschungsresultate der Sinnesphysiologie, so beeindruckend und exakt sie sein mögen, nicht die objektive Wahrheit darstellen, denn die Messungen, die wir als Sinnes- 
physiologen machen, finden wiederum in unserer Wahrnehmungswelt und damit unter ihren Bedingungen statt. Wir können diesen Bedingungen nicht gänzlich entfliehen, denn schließlich müssen wir Zeiger ablesen, Zahlenkolonnen durchgehen und Grafiken interpretieren, und all dies geschieht in unserer Sinneswelt. Was wir aber tun können, ist nichts anderes als eine zweite Wahrnehmungswelt zu schaffen, die genauer und standardisierter ist als die erste und zumindest anders aufgebaut, und die wir „naturwissenschaftlich“ nennen. Diese beruht auf Messmethoden und Methoden der Hypothesen- und Theoriebildung und ihrer Überprüfung.

Wir sind entsprechend in der Lage, diese beiden Welten, die der unmittelbaren Wahrnehmungsinhalte und die der mithilfe naturwissenschaftlicher Messungen erfassten Ereignisse, miteinander zu vergleichen und festzustellen, inwieweit sie zusammenhängen. Wir wissen bereits, dass dieser Zusammenhang meist lose und manchmal gar nicht direkt vorhanden ist, wenn es nämlich physikalische oder chemische Ereignisse gibt, auf die unsere Instrumente reagieren, unsere Sinnesorgane aber nicht, oder wenn unsere Sinnesorgane und die nachgeschalteten Systeme im Gehirn Wahrnehmungen hervorbringen, denen gar keine physikalischen oder chemischen Reize entsprechen, wie dies bei den Farb- und Bewegungsnachbildern der Fall ist.

\section{Wie verlässlich arbeiten unsere Sinnessysteme?}

Mithilfe geeigneter Verfahren können wir die Eigenschaften des Wahrnehmungsapparates genauer studieren, der zwischen der (als existent angenommenen) Welt und unseren Wahrnehmungen liegt. Nach herkömmlicher Anschauung wird dieser Wahrnehmungsapparat von physikalischen und chemischen Ereignissen der Welt erregt, und diese Erregungen rufen dann unsere Wahrnehmungserlebnisse hervor. Sollte die Anschauung des kritischen Realisten zutreffen, dass unsere Wahrnehmungen deshalb überlebensfördernd sind, weil sie die Ereignisse der Welt - zumindest die überlebensrelevanten - mehr oder weniger zutreffend wiedergeben, so müsste dies in einer mehr oder weniger verlässlichen Arbeit des Wahrnehmungsapparates erkennbar sein, ohne dass wir von einem strikten Abbildcharakter der Wahrnehmung ausgehen müssen.

Unsere Sinnessysteme sind eine Art Berichterstatter über die Welt. Wie verlässlich arbeiten aber unsere Sinnessysteme? Nehmen wir hierzu als Beispiel das visuelle System, das am besten von allen Sinnessystemen untersucht ist. Um seine Verlässlichkeit zu beurteilen, müssen wir uns vergegenwärtigen, aus welchen Merkmalen unsere visuellen Wahrneh- 
mungen überhaupt bestehen. Die grundlegendste Eigenschaft ist natürlich, dass es sich um einen Seheindruck handelt und nicht um Hören, Tasten, Riechen und Schmecken. Dies nennt man die spezifische Sinnesmodalität. Innerhalb dieser Sinnesmodalität des Sehens gibt es nun Helligkeiten, Farben und Bewegungen bzw. Bewegungsrichtungen, die man primäre visuelle Qualitäten nennen kann. Darüber hinaus gibt es sekundäre Qualitäten, die sich aus den primären Qualitäten durch Kombination und Vergleich zusammensetzen wie Helligkeits- und Farbkontraste, Bewegungsmuster und Bewegungsgeschwindigkeiten und deren Abänderungen sowie den Ort dieser Ereignisse. Hieraus wiederum ergeben sich tertiäre Qualitäten wie Konturen, Gestalten und räumliche Tiefe sowie komplexe dreidimensionale Anordnungen von ruhenden und bewegten farbigen oder unbunten Gestalten, also ganze Szenen. Diese Szenen sind dann die eigentlichen Inhalte unserer visuellen Wahrnehmung.

Sollte der kritische Realist recht haben, so müsste es für all diese Inhalte hinreichend verlässliche Entsprechungen zwischen drei Instanzen und nicht nur zwei geben, nämlich der Welt, so wie wir sie mit unseren Messinstrumenten erfassen, dem visuellen Wahrnehmungssystem und unseren subjektiven Seheindrücken, denn diese Seheindrücke entstehen ja nicht direkt aus den Einwirkungen der Welt auf die Sinnesorgane, sondern aus den Erregungszuständen der Sinnessysteme, die ihre Botschaften über die sensorischen Nerven ins Gehirn senden.

Am ehesten stellen wir eine systematische Entsprechung auf der Ebene der primären visuellen Qualitäten fest. Studieren wir die Aktivität der Photorezeptoren in unserer Netzhaut und der nachgeschalteten visuellen Neurone, so stellen wir fest, dass zumindest einige von ihnen relativ verlässlich auf Veränderungen der physikalischen Helligkeit reagieren, zumindest in einem weiten Bereich, der von einer sternklaren Nacht bis zur Mittagssonne im Sommer reicht. Die Erregung durch eine selbststrahlende oder reflektierende Lichtquelle nimmt in einer annähernden logarithmischen Funktion in dem Maße zu, wie die Zahl der von den Photorezeptoren pro Zeiteinheit aufgenommenen Lichtquanten zunimmt. Dies empfinden wir dann als Zunahme der Helligkeit, und zwar so, dass im Bereich geringer Lichtverhältnisse Unterschiede stärker wahrgenommen werden als im Bereich grellen Lichts. Diese Tatsache ist im so genannten Weber-Fechner-Gesetz ausgedrückt.

Unter natürlichen Bedingungen ist allerdings die Helligkeitswahrnehmung komplizierter und hängt nicht von der absoluten, sondern von der relativen Intensität des Lichtes ab, das von Oberflächen reflektiert wird. Um dies zu überprüfen, nehmen wir ein helles und ein dunkles 
Stück Karton in die Hand und messen in heller Sonne die Intensität des reflektierten Lichtes. Bei unseren Messungen stellen wir natürlich fest, dass der helle Karton sehr viel mehr Licht reflektiert als der dunkle. Wenn wir nun die Messung in der Dämmerung wiederholen, dann messen wir, dass der helle Karton nach wie vor eindeutig heller aussieht als der dunkle, aber jetzt viel weniger Licht reflektiert als der dunkle Karton im Tageslicht. Wenn die subjektive Helligkeit eindeutig von der absoluten Menge reflektierten Lichtes abhinge, dann müsste die Fläche jetzt dunkel aussehen, was sie aber nicht tut. Das helle bzw. dunkle Aussehen kann also gar nicht direkt mit der absoluten Menge des reflektierten Lichtes zusammenhängen.

Des Rätsels Lösung besteht darin, dass bei Dämmerung der dunkle Karton noch viel weniger Licht abstrahlt als der helle. Das Verhältnis der Menge des von einer Oberfläche reflektierten Lichtes unterschiedlicher Oberflächen bewirkt also, ob sie hell oder dunkel erscheinen, und zwar jeweils „korrigiert“ in Bezug auf die Umgebungshelligkeit. Es erscheinen diejenigen Oberflächen am hellsten, die bei einer gegebenen Gesamthelligkeit das meiste Licht reflektieren, und diejenigen am dunkelsten, die dies am wenigsten tun.

Über den Zusammenhang zwischen der zweiten Grundqualität des Lichtes, seiner Wellenlänge, und der Farbwahrnehmung haben wir bereits viel gehört. Wenn wir in einem visuellen Experiment die spektrale Zusammensetzung eines Lichtreizes von kurzwellig nach langwellig ändern, dann erleben wir einen Übergang von Blauviolett über Grün, Gelb und Orange nach Rot. Wir müssen aber auch berücksichtigen, dass wir jede Farbwahrnehmung durch eine fast beliebige Farbaddition und -subtraktion hervorrufen können. Dabei gibt es - wie jeder Farbenpraktiker weiß - zu Hauf unvorhersehbare Effekte. Wir können also von einer subjektiven Farbwahrnehmung nicht verlässlich auf das Wellenlängenspektrum schließen, das von der Oberfläche eines Gegenstandes reflektiert wird.

Mit der Wahrnehmung der primären Qualitäten Lichtintensität und Wellenlänge, denen subjektiv Helligkeit und Farbe entsprechen, hat es sich aber auch schon, denn nur auf die Intensität (bzw. deren Änderung) und die Wellenlänge des Lichtes können die einzelnen Photorezeptoren unserer Netzhaut reagieren. Bei der dritten primären visuellen Qualität, der Bewegung, kommen wir bereits in Schwierigkeiten, denn ein einzelner Photorezeptor kann gar keine Bewegung wahrnehmen (genauso wenig wie ein einziger Photorezeptortyp Farbe wahrnehmen kann), das können nur mehrere Photorezeptoren, nämlich mindestens zwei, die auf 
eine bestimmte Weise mit einer nachgeschalteten Nervenzelle verbunden sind. Diese Verschaltung, „Bewegungsdetektor“ genannt, sorgt dafür, dass die nachgeschaltete Nervenzelle (vornehmlich eine Retinaganglienzelle) dann erregt wird, wenn die ihr vorgeschalteten Photorezeptoren nacheinander durch Lichtpunkte gereizt werden. Verbindet man eine kleine Fläche von Photorezeptoren mit einer Retinaganglienzelle und verschaltet sie in besonderer Weise, dann kann man neben der Bewegung als solcher auch die Geschwindigkeit und die Richtung bzw. die Bahn „errechnen“. Wir sehen also, dass so einfache visuelle Merkmale wie Bewegung, Bewegungsrichtung und Geschwindigkeit gar nicht primär gegeben sind, sondern von Netzwerken „errechnet“ bzw. konstruiert werden. Es handelt sich allerdings um ziemlich einfache Konstruktionen.

Kontraste zwischen unterschiedlichen Helligkeiten und Farben sind eine wichtige Grundlage der Objektwahrnehmung: Wo es keine Kontraste gibt, nehmen wir auch keine Gegenstände und Gestalten wahr. Die neuronalen Verschaltungen, die der Helligkeits- und Farbkontrastwahrnehmung zugrunde liegen, sind ebenfalls nicht besonders kompliziert; sie benötigen die bereits genannten ,antagonistisch“ arbeitenden Nervenzellen in unserer Netzhaut und im nachgeschalteten visuellen System des Gehirns. Kontraste allein ergeben aber noch keine Gestalten bzw. Objekte, sondern erst dann, wenn sie sich in einer ganz bestimmten Weise zusammenfügen, zum Beispiel wenn Linien oder Farben eine Fläche begrenzen. Dies geschieht in unserem Sehsystem meist völlig automatisiert, ja geradezu zwanghaft, gelegentlich sehen wir Kontraste auch dort, wo sie physikalisch gar nicht vorhanden sind, wie dies bei der bekannten Kanizsa-Täuschung mit ihren Scheinumrissen der Fall ist. Die Netzwerke, die mit Gestaltwahrnehmung befasst sind, konstruieren sie automatisch hinzu. Sehr schön sehen wir dies, wenn wir in die Wolken schauen und überall Gestalten und Gesichter entdecken.

Am deutlichsten sehen wir Objekte, wenn sich Konturen vor einem ruhenden Hintergrund bewegen. Ruhende Objekte haben die Tendenz, mit ihrer Umgebung zu verschmelzen und unsichtbar zu sein (dies ist der Grund dafür, dass sich Tiere und Menschen, die nicht gesehen werden wollen, ganz ruhig verhalten). Bewegung vor einem Hintergrund lässt eine Gestalt geradezu hervorspringen. So starren die von mir ausführlich untersuchten Salamander oft lange eine Grille an, an die sie sich auf Schnappdistanz angenähert haben und die so „schlau“ ist, sich nicht mehr zu bewegen. Sie schnappen aber blitzschnell zu, sobald sich die Grille wieder regt (oder auch nur ihre Fühler sich bewegen). 
Objekterkennung über Bewegung geschieht im Sehsystem meist völlig automatisiert, aber dies ist beim Salamander wie bei uns bereits eine ziemlich komplizierte Angelegenheit. Hierbei müssen nicht nur bestimmte Ansammlungen von Bildpunkten als Gestalt interpretiert werden, was meist Erfahrung voraussetzt, sondern bei Bewegungen verformt sich diese Gestalt häufig stark, besonders wenn es sich um belebte Objekte wie eine Grille oder eine Person handelt. Wenn mein Salamander die von ihm verfolgte Grille einmal von der Seite, dann wieder von vorn oder von hinten sieht, einmal auf die laufenden Beine, ein andermal auf die sich bewegenden langen Fühler schaut, dann muss sein visuelles System ziemlich komplizierte Transformationsberechnungen anstellen, um die Identität des Objekts festzustellen. Dasselbe geschieht bei uns, wenn wir eine bestimmte Person durch ein Dickicht oder in einer Menschenmenge verfolgen. Ist es noch dieselbe Person, die da wieder auftaucht, oder eine andere?

Ein anderer grundlegender visueller Wahrnehmungsinhalt, nämlich räumliche Tiefe, beruht ebenfalls auf komplizierten Berechungen unseres visuellen Systems mithilfe ganz unterschiedlicher Hilfsmittel (retinale Disparität, Bewegungsparallaxe, Linsenakkomodation, Texturgradienten und Helligkeit), ohne dass wir hiervon irgendetwas merken, und ebenso wenig merken wir etwas von dem großen Aufwand, den unser Gehirn treiben muss, um eine stabile visuelle Umwelt zu konstruieren. Wir merken deshalb nichts davon, weil die daran beteiligten visuellen Netzwerke - nach allem, was wir wissen - dies aufgrund von Schaltungen leisten, die entweder genetisch determiniert sind oder sich in einem sehr frühen Entwicklungsstadium verfestigen. Dennoch handelt es sich um Konstrukte, die keinerlei Abbilder der Welt sind.

Dies heißt, dass Bewegungen, Farben, Formen und der uns umgebende Raum nicht direkt von den Bewegungen, Wellenlängenunterschieden, Kontrasten und räumlichen Anordnungen in der Welt abgeleitet, sondern das Produkt von Berechnungen in neuronalen Netzwerken sind. Da uns diese Wahrnehmungen verlässlich gegeben sind, halten wir sie fälschlich für Zustände der bewusstseinsunabhängigen Welt. Auch unsere sinnesphysiologischen und physikalischen Messungen sagen uns letztlich nicht, welche objektiven Vorgänge unseren Wahrnehmungen zugrunde liegen, sie zeigen uns nur, dass zwischen dem gemessenen physikalischen (oder chemischen) Reiz und unseren Wahrnehmungsinhalten keine irgendwie geartete Ähnlichkeit herrscht. Wenn man den Physiker fragt, was Bewegung, Raum, Zeit oder Ursache wirklich ist, dann wird er - sofern er philosophisch vorgebildet ist - dies als keine 
sinnvolle Frage ansehen, sondern auf Gleichungen deuten, die helfen, Phänomene in systematischer und logisch konsistenter Weise zu interpretieren.

\section{Die Unspezifität neuronaler Aktivität und der Ortscode}

Die Sinnesmodalität, also der erlebte Unterschied zwischen Sehen, Hören, Tasten, Riechen und Schmecken, ist sicher das wichtigste Merkmal der Sinnesreize. Wie kommt die Reizmodalität zustande? Eigentlich sollte dies kein großes Problem sein, denn alles, was vom Auge über den Sehnerven ins Gehirn gelangt, ist Sehen, und entsprechendes sollte für das Ohr und Hören, die Haut und Tasten, die Riechschleimhaut und Riechen, die Zunge und Schmecken gelten. An einfachsten wäre es für das Gehirn, wenn die verschiedenen Sinnesmodalitäten sich durch ganz unterschiedliche Erregungszustände etwa nach Art eines Farbcodes zu erkennen gäben.

So etwas ist lange Zeit tatsächlich angenommen worden, aber diese Annahme ist falsch. Registrieren wir beim Sehen die neuronale Aktivität in der visuellen Rinde und vergleichen sie mit derjenigen in der Hörrinde beim Hören und tun entsprechendes beim Tasten, Riechen und Schmecken, dann können wir in der neuronalen Aktivität keinerlei Unterschiede feststellen. Die Aktionspotentiale und die graduierten Potentiale der aktivierten Nervenzellen sind dieselben in den verschiedenen Sinnessystemen, und dasselbe gilt für die neurochemischen Vorgänge an den Synapsen. Es gibt überhaupt keine neuronale Aktivität, die von ihrer Beschaffenheit für Sehen, Hören, Tasten, Riechen, Schmecken spezifisch wäre, für die einzelnen Farben, für Melodien, Druck und Schmerz, und dasselbe gilt auch für solche neuronalen Aktivitäten, die mit Denken, Vorstellen und Erinnern zu tun haben.

Dies ist die „Unspezifität“ oder „Neutralität“ neuronaler Erregungen gegenüber ihren Inhalten. Sie hat ihren Entdeckern im 19. Jahrhundert großes Kopfzerbrechen bereitet, aber der größte unter ihnen, der Physiker und Physiologe Hermann von Helmholtz, fand die Lösung des Rätsels. Sie lautet, dass Modalitäten und Qualitäten von Sinnesreizen durch den Ort der Verarbeitung im Gehirn festgelegt werden und nicht durch die Beschaffenheit der damit verbundenen neuronalen Aktivität. Dies kann man dadurch beweisen, dass man mit derselben (unschädlichen und schmerzfreien) elektrischen Erregung mithilfe einer Reizelektrode im visuellen Cortex visuelle Halluzinationen, im auditorischen 
Cortex auditorische und im somatosensorischen Cortex somatosensorische Empfindungen hervorruft - meist allerdings nur ziemlich einfache. Dieses Prinzip gilt übrigens auch für subcorticale Zentren: Elektrische Stimulation von Teilen des Hypothalamus ruft Wut hervor, Erregung von Teilen der Amygdala Furcht, von Teilen des mesolimbischen Systems Lustgefühle usw. Der Ort der Erregung legt den Inhalt fest, und zwar unabhängig davon, woher die Erregung stammt.

Die Gültigkeit dieses Ortsprinzips oder Ortscodes wird dadurch gewährleistet, dass das Gehirn sich in seinem Wachstum in einer ganz bestimmten Weise verknüpft, die - von Fehlentwicklungen abgesehen dafür sorgt, dass die von der Netzhaut stammende Erregung über den Thalamus zum Hinterhauptslappen (Okzipitalcortex) gelangt, die vom Innenohr stammende Erregung über das Mittelhirndach und den Thalamus zum Schläfenlappen (Temporalcortex) und die von der Haut und den Muskeln stammende Erregung wiederum über den Thalamus zum vorderen Scheitellappen (Parietalcortex). Verändert man in einem sehr frühen Entwicklungsstadium diese Verbindungsbahnen und lässt die Erregungen vom Auge im Temporalcortex und die vom Ohr im Okzipitalcortex enden (solche Experimente lassen sich nur bei ganz bestimmten Versuchstieren machen), dann sieht das Tier mit dem für das Hören vorgesehenen Cortex und umgekehrt (zumindest verhält es sich so, als ob es etwas sähe bzw. hörte). Etwas Ähnliches findet im Übrigen bei Personen statt, die sehr früh erblindet sind. Hier dehnt sich der somatosensorische Cortex des Scheitellappens in den Bereich des „,nutzlos“ gewordenen visuellen Cortex des Hinterhauptslappens hinein aus, und es kommt zu einer Uminterpretation der corticalen Aktivität. Insgesamt zeigen uns diese Befunde, dass im Gehirn der Kontext, in dem eine Erregung stattfindet, wichtig für ihre Bedeutung ist.

Eine Konstruktionsebene ganz neuer Art betreten wir, wenn wir die große Erfahrungsabhängigkeit unserer Wahrnehmung berücksichtigen. Dass unsere Wahrnehmungsleistungen zum Teil in dramatischer Weise von unserer Erfahrung abhängen, merken wir, wenn wir uns in neue Umgebungen begeben und dann längere Zeit benötigen, um uns wahrnehmungsmäßig darin zurechtzufinden. Anfangs sind wir wie blind und haben Mühe, all die Dinge und ihre spezifischen Anordnungen zu erkennen. Später, wenn wir mit dieser Umgebung vertraut sind, sehen wir mit einem Blick, dass alles an seinem Platz ist. Untersuchen wir diesen Vorgang genauer, so erkennen wir, dass wir vertraute Umgebungen wie unser Arbeits- oder Wohnzimmer gar nicht mehr Punkt für Punkt wahrnehmen, sondern dass unserem visuellen System wenige Anhaltspunkte 
genügen, um ein vollständiges Bild der Umgebung zu konstruieren, und zwar aus dem Gedächtnis heraus. Wir merken davon meist nichts, und dies hat die zuweilen verhängnisvolle Konsequenz, dass wir Abweichungen vom Gewohnten völlig übersehen.

Viele erfahrungs- und gedächtnisgeleiteten Prozesse finden allerdings in früher Jugend statt, zum Teil unmittelbar nach der Geburt. Unser Gehirn lernt dabei, wie die visuelle Welt aufgebaut ist, d.h. wie Objekte sich hinsichtlich ihrer Helligkeit, Farbe, Form und Bewegung unterscheiden, dass sie nicht wirklich verschwinden, wenn sie nicht mehr sichtbar sind, dass sie dieselben bleiben, auch wenn sie sich ändern usw. Das Gehirn lernt Gesichter und die Körper und ihre Bewegungen unterscheiden und so fort. Dieses Lernen geschieht auf eine sehr schnelle und exemplarische Weise, weil hierfür jeweils spezifische, genetisch vorgegebene Netzwerke existieren, die nur darauf warten, „informiert“ zu werden. Sie verfestigen sich mehr und mehr und können später nur mit großem Aufwand verändert werden, so dass ihre Leistungen wie angeboren aussehen. Wachsen Tiere und Menschen jedoch in Umgebungen auf, in denen bestimmte Mindestinformationen über Farben, Gestalten und Bewegungen nicht vorhanden sind, dann zeigen sich schwere Defizite der Wahrnehmung, denn es fehlte den Sinnessystemen das nötige Reizangebot zur richtigen Entwicklung.

\section{Neurobiologischer und radikaler Konstruktivismus}

Wir können deshalb sagen, dass bei komplexen Wahrnehmungen unser Gedächtnis das wichtigste Wahrnehmungsorgan ist. Aufbauend auf genetisch vorgegebenen oder früh verfestigten primären Interpretationshilfen wie den oben genannten ist jeder Wahrnehmungsprozess eine Hypothesenbildung über Gestalten, Zusammenhänge und Bedeutungen der Welt. Anders ausgedrückt: Die Art und Weise, wie im Prozess der Wahrnehmung unsere Umgebung in bedeutungsvolle Gestalten und Geschehnisse gegliedert wird, ist eine Folge von Versuch und Irrtumsbeseitigung, von Konstruktions- und Interpretationsversuchen, von Bestätigung und Korrektur.

Diese Auffassung entspricht derjenigen des erkenntnistheoretischen Konstruktivismus. Dieser betont, dass es keinen direkten Zusammenhang zwischen den Vorgängen in der Welt und den Inhalten unserer Wahrnehmung gibt. Die Vorgänge in der Welt bilden sich nicht direkt im Gehirn ab, sondern bewirken Erregungen in den Sinnesorganen, die zur Grundlage von Konstruktionsprozessen unterschiedlicher Komplexität 
und Beeinflussung durch Lernprozesse werden, an deren Ende oft, aber keineswegs zwangsläufig, unsere bewussten Wahrnehmungsinhalte stehen. Aus den Wahrnehmungsinhalten selbst lässt sich umgekehrt nicht die Beschaffenheit der bewusstseinsunabhängigen Welt erschließen, weil das, was „von draußen“ kommt, sich von dem, was das konstruktive Gehirn „hinzu tut“, nicht verlässlich unterschieden werden kann - beides ist ja nur innerhalb des Gehirns vorhanden! Diese Anschauung wird durch die sinnes- und neurophysiologische Forschung voll bestätigt und ist praktisch neurobiologisches Gemeingut geworden.

Von diesem neurobiologisch-psychologisch inspirierten Konstruktivismus muss man den so genannten radikalen Konstruktivismus unterscheiden. Dieser radikale Konstruktivismus erweckt den Eindruck, als gebe es im Gehirn eine Instanz, die sich bewusst Modelle über die „Welt da draußen" macht, sie ausprobiert und sich gleichzeitig fragt, ob es diese Welt überhaupt gibt. Manche radikalen Konstruktivisten halten selbst diese Frage für sinnlos, weil sie objektiv nicht zu beantworten sei. Jede Aussage über die Existenz einer bewusstseinsunabhängigen Welt sei eine Aussage in der Bewusstseinswelt. Dieser Standpunkt wird in einem Zitat des Hauptvertreters des radikalen Konstruktivismus, Ernst von Glasersfeld, deutlich, das lautet: „Der radikale Konstruktivismus beruht auf der Annahme, dass alles Wissen, wie immer man es auch definieren mag, nur in den Köpfen von Menschen existiert und dass das denkende Subjekt sein Wissen nur auf der Grundlage eigener Erfahrung konstruieren kann. Was wir aus unserer Erfahrung machen, das allein bildet die Welt, in der wir bewusst leben" (von Glasersfeld, 1996).

Anders ausgedrückt: Was für einen Beobachter wie die Wahrnehmung externer Geschehnisse aussieht, ist in Wirklichkeit ein Prozess der internen Hypothesenbildung über die möglichen Bedeutungen der intern erfahrenen Veränderungen. Das System versucht dabei, bestimmte interne Mängel-, Bedürfnis- oder Ungleichgewichtszustände auszugleichen. Die Umwelt existiert aber für das System nicht real, sondern ebenfalls nur als Konstrukt

Richtig an diesem Standpunkt ist, dass das Nervensystem bzw. Gehirn keine Information im Sinne von Bedeutung und Wissen aufnehmen kann. Bedeutung entsteht, indem Umweltreize Erregungen in den Sinnesorganen hervorrufen, die mithilfe unterschiedlichster Mechanismen und auf den unterschiedlichsten Ebenen des Nervensystems und Gehirns miteinander verglichen und verrechnet und zunehmend mit Gedächtnisinhalten versetzt werden. Dennoch ist es falsch, diesen Konstruktionsprozess als eine Art bewusster Hypothesenbildung zu sehen, wie er in der 
Wissenschaft abläuft, wo man Daten hin und her wälzt, bis sie sich möglichst widerspruchsfrei und mit dem höchsten Erklärungsgrad zusammenfügen. Wir müssen über unsere Wahrnehmungskonstrukte nicht nachdenken, und sie unterliegen auch nicht unserem Willen. So etwas würde uns zu Konstrukten verleiten, die gegebenenfalls „lebensgefährlich“" wären. Vielmehr läuft unsere Wahrnehmung, auch die erfahrungsgeleitete, hochautomatisiert ab.

Wenn unsere Wahrnehmungen also Konstrukte sind (wenngleich keine bewusst-willkürlichen) und keine Abbilder, wieso sind sie trotzdem in aller Regel verlässlich? Dies können wir am ehesten verstehen, wenn wir uns klarmachen, dass nicht unsere Wahrnehmungen ,richtig“ sein müssen, sondern unsere Verhaltensweisen. Man kann zeigen, dass zwischen den Gegebenheiten in der Umwelt und den Verhaltensweisen eine Passung vorhanden sein muss in dem Sinne, dass das Leben und Überleben in unserer natürlichen und sozialen Umwelt nach externen Kriterien der physischen Fortdauer und internen Kriterien des biologischen und psychischen Wohlbefindens sichert. Ein Salamander muss nicht die Welt korrekt erkennen, um eine Fliege zu fangen, und wir müssen den physikalischen Raum nicht so abbilden, wie er tatsächlich ist, um uns in ihm zurechtzufinden. Es genügen - aus der Sicht des Beobachters - Annäherungsmodelle, die, wenn es darauf ankommt, verfeinert werden, so dass eine präzisere Verhaltenssteuerung möglich ist.

All dies ist in unseren Sinnessystemen und in unserem Gehirn vor vielen Millionen von Jahren geschehen, und deshalb sind diese Konstrukte so verlässlich. Andere Konstrukte erhalten ihre Verlässlichkeit über die sich verfestigenden Lernprozesse während der Frühstadien unserer Entwicklung, die zudem von stammesgeschichtlich bewährten Regeln geleitet werden. Anderes schließlich unterliegt dem Spiel der aktuellen Hypothesenbildung und Konstruktion, wenn wir mit neuen Gesichtern, Szenen, Sätzen und Sachverhalten konfrontiert werden und deren Bedeutung erfassen müssen. Aber auch dies geschieht in aller Regel unbewusst und automatisiert und immer unter Zuhilfenahme bewährten Gedächtnis-Materials. Das macht diese Konstrukte ebenfalls vergleichsweise verlässlich. Wir haben also in der Wahrnehmung einen Stufenbau von Konstruktionsebenen vorliegen, die teilweise erfahrungsbedingt sind, aber meistenteils nicht von unserem Bewusstsein und unserem Willen abhängen und deshalb so verlässlich arbeiten. Sie müssen deshalb nicht im metaphysischen Sinne ,wahr“ sein.

Interessant ist es zu fragen, ob und inwieweit der hier vorgetragene neurobiologische Konstruktivismus sich von einem kritischen Realismus 
oder gar einem hypothetischen Realismus, wie ihn Gerhard Vollmer vertritt, sich überhaupt unterscheidet. In vielen Grundaussagen, insbesondere dem Hinweis auf die evolutionäre Bedingtheit unseres „Erkenntnisapparats“, auf die „Passung“ zwischen diesem Apparat und den speziellen Lebensbedingungen und der hohen Plausibilität der Annahme der Existenz einer bewusstseinsunabhängigen Welt gibt es keine oder nur geringe Unterschiede. Die wirklich gravierenden Unterschiede liegen nicht im ontologischen, sondern im erkenntnistheoretischen Status der „realen“, bewusstseinsunabhängigen Außenwelt. Beide Positionen, der neurobiologische Konstruktivismus und der kritische bzw. hypothetische Realismus gehen von der Existenz einer bewusstseinsunabhängigen Welt aus. Der kritische bzw. hypothetische Realismus glaubt bei aller Anerkennung des hypothetischen Charakters dieser Annahme von der Korrektiv-Funktion der Realität aus, insbesondere wenn - wie Vollmer betont - Hypothesen scheitern. Idealismus oder Konstruktivismus könnten - so Vollmer - zwar das Bestätigen von Hypothesen, nicht aber ihr Scheitern erklären, und dieses Scheitern sei ebenso wichtig für den Erkenntnisfortschritt wie das Bestätigen. Während letzteres zweifellos richtig ist, trifft ersteres nicht zu. Um das Scheitern einer Hypothese festzustellen, benötigt man -zumindest in der modernen Forschung - ebenso umfangreiche methodische und begriffliche Vorannahmen wie für ihre Bestätigung. Viele Hypothesen der neuzeitlichen Wissenschaft sind nur scheinbar gescheitert und haben sich - meist nach einigen Modifikationen oder Verbesserungen in den Prüfverfahren - als richtig erwiesen. In der berühmten Konkurrenz des ptolemäischen und des kopernikanischen Bildes unseres Sonnensystems war hinsichtlich der Exaktheit der Vorhersage der Planetenstellungen das komplizierte ptolemäische mit seinen Zykeln und Epizykeln dem kopernikanischen weit überlegen und hätte demnach jede empirische Überprüfung gewonnen. Die ursprüngliche Theorie von John Eccles, dass die Nervensynapse ausschließlich elektrischer Natur ist, wurde nicht durch direkte Experimente entschieden, sondern durch eine umfassende Gewichtung und Interpretation einer Vielzahl widerstreitender experimenteller Befunde. Übrigens hat der Philosoph Karl Popper (der „Erfinder“ des Falsifikationismus) bei der „Konversion" von Eccles zur chemischen Natur der Synapse eine große Rolle gespielt. Ironischerweise hatte Eccles doch zum Teil recht, denn heute weiß man, dass es überall im Gehirn neben den chemischen Synapsen auch elektrische gibt - vielleicht sogar viel mehr als die ersteren.

Mögen wir also noch so fest an die Existenz einer bewussteinsunabhängigen Welt glauben - und dieser Glaube ist für einen Biologen 
wohl unabdingbar -, so hilft dies uns bei der Frage nach der Verlässlichkeit unserer Sinnesempfindungen und Wahrnehmungen nicht weiter. Was bleibt, ist der systematische Vergleich von Wahrnehmungen untereinander und die Prüfung unserer Aussagen auf Widersprüchlichkeit. Eine bewusstseinsunabhängige Welt wäre eben nicht bewusstseinsunabhängig, wenn sie - und sei es auch nur partiell - erkennbar wäre. Dies verbietet uns selbstverständlich nicht, dass wir uns möglichst vernünftige und plausible Annahmen über den Aufbau und die Gesetze dieser Welt machen.

\section{Weiterführende Literatur}

Dudel, J./Menzel, R./Schmidt, R.F. (Hrsg.): Neurowissenschaften. Vom Molekül zur Kognition, Berlin/Heidelberg/New York 2001.

Kandel, E. R./Schwartz, J. H./Jessell, T. M.: Neurowissenschaften, Heidelberg 1996.

Kutschera, F. von: Grundfragen der Erkenntnistheorie, Berlin 1982.

Roth, G.: Das Gehirn und seine Wirklichkeit, Frankfurt a.M. 2000.

Roth, G.: Fühlen, Denken, Handeln, Frankfurt a.M. 2003.

Vollmer, G.: Evolutionäre Erkenntnistheorie, Stuttgart 1975. 

ECKART VOLAND

\section{DAS, HANDICAP-PRINZIP' UND DIE BIOLOGISCHE EVOLUTION DER ÄSTHETISCHEN URTEILSKRAFT}

\section{Einführung}

Wie immer man die ästhetische Urteilskraft im Einzelnen definieren mag, auf der psychologischen Ebene gründet sie ganz wesentlich auf der Einschätzung von Dingen und Szenarien als ,anziehend“ bzw. „,abstoßend“. Das Schöne zieht an, das Hässliche stößt ab, womit sich das ästhetische Urteil als Ausdruck einer psychischen Präferenz darstellt. Präferenzen stehen aber im Dienst evolvierter psychischer Mechanismen, deren adaptive Funktion in der Entscheidungsfindung angesichts von Lebens- und Überlebensproblemen besteht. Indem sie relevante Information verarbeiten, helfen Präferenzen adaptive Probleme als solche zu erkennen und jeweils vorteilhafte Lösungen zu motivieren. ${ }^{1}$ Die evolutionäre Funktionslogik einiger Präferenzen ist recht gut verstanden. Die Tendenz zur Bevorzugung frischer, aromatischer Speisen, sicherer und ökologisch produktiver Orte, angenehmer Klimate und sexuell attraktiver Partner beispielsweise lässt sich gut als evolutionär kumuliertes Ergebnis fitnessförderlicher Entscheidungen im Zuge von Nahrungs-, Habitat-, bzw. Partnerwahl nachvollziehen. Ästhetisches Urteilsvermögen ist in diesen Fällen als Überlebens- und Reproduktionshilfe funktional: Was uns anzieht, bietet Chancen der Fitnesssteigerung, was uns abstößt, birgt Fitnessrisiken. ${ }^{2}$ Die Evolution dieser Präferenzen lässt sich problemlos darwinisch verstehen: Diejenigen unter unseren Vorfahren, die über ein differenzierteres ästhetisches Urteilsvermögen verfügten und kompetenter damit umgehen konnten, haben mit größerer Wahrscheinlichkeit erfolg-

1 Buss, D. M.: Evolutionary Psychology - The New Science of the Mind, Boston 1999.

2 Thornhill, R.: „Darwinian aesthetics informs traditional aesthetics“, in: Voland, E./Grammer, K. (Hrsg.): Evolutionary Aesthetics, Heidelberg 2003, S. 9-35. 
reich reproduziert und damit ihre Anlagen für ästhetische Kompetenz dem genetischen Profil unserer Art beigefügt.

Ästhetik würde sicherlich nicht dieses nach wie vor weitgehend theorieresistente Riesenproblem der Evolutionsbiologie bilden, wenn sie auf die genannten Bereiche beschränkt bliebe, denn hier, beim Verständnis von Nahrungs-, Habitat- und Partnerwahl gibt es Einsichten von der Art, wie Evolutionsbiologen sie suchen. Wer wollte in diesen Zusammenhängen die biologische Funktionalität ästhetischer Präferenzen bestreiten? Allerdings - und daraus erwächst das eigentliche Problem sind es nicht nur die ,spontanen“ Merkmale der vorfindlichen Lebenswelt, die uns anziehen oder abstoßen, sondern auch intentionierte Merkmale einer artifiziellen Kunstwelt. Überall auf der Welt engagieren sich Menschen mit dem, was Dissanayake ${ }^{3}$ als making special bezeichnet hat. Sie verbrauchen Zeit, Energie und Ressourcen, nur um die Dinge um sich herum anders als gewöhnlich erscheinen zu lassen. Die Ergebnisse des making special werden mit den Attributen von schön oder hässlich belegt, weil sie uns anziehen oder abstoßen. Aber welchen biologischen Sinn macht dieses Urteil? Auf den ersten Blick scheint es wenig plausibel, dass auch in artifiziellen Kontexten ästhetische Präferenzen als Orientierungshilfe in fitnessrelevanten Lebensentscheidungen dienen könnten. Und wenn aber doch, in welchen? Oder hat sich stattdessen die Ästhetik der Kunstwelt von der „Tyrannei der Funktionalität ${ }^{\text {“4 }}$ befreit - und damit zugleich kreative Freiräume für die Tyrannei eigenwilliger Meme geschaffen?

\section{2. Ästhetisches Urteilsvermögen in der Welt der Artefakte: Evolutionäre Angepasstheit oder funktionsloses Nebenprodukt?}

Die Geschichte biologischer Merkmale kann sich grundsätzlich auf zwei unterschiedlichen Routen vollziehen. Entweder ein Merkmal verdankt seine evolutionäre Persistenz einem spezifischen Selektionsvorteil - in diesem Fall handelt es sich um eine Angepasstheit - oder aber das Merkmal wird als nichtfunktionales Nebenprodukt einer anderen funktionalen Angepasstheit mit durch die evolutionären Zeiträume geschleppt. Dass Knochen weiß sind, ist keine Angepasstheit, weil die Farbe der Knochen

3 Dissanayake, E.: Homo Aestheticus: Where Art Comes From and Why, New York 1992.

4 Lumsden, C. J.: „Aesthetics“, in: Maxwell, M. (Hrsg.): The Sociobiological Imagination, Albany 1991, S. 253-268. 
niemals selektiert wurde. Stattdessen sind Kalziumeinlagerungen ins Knochengewebe biologisch funktional und angepasst, und Kalzium ist nun mal weiß. Die Knochenfarbe ist deshalb ein ,zufälliges“ Nebenprodukt eines aus anderen Gründen adaptiven Zusammenhangs. Bezogen auf unser Thema stellt sich für ein evolutionäres Verständnis ästhetischer Präferenzen die höchst bedeutsame Frage, ob sie in der Welt intentionierter Artefakte nun funktionslose Nebenprodukte oder biologisch funktionale Angepasstheiten sind.

Dabei gibt es theoretisch zwei Möglichkeiten, in denen uns das ästhetische Urteilsvermögen als nichtselektiertes und deshalb funktionsloses Nebenprodukt gegenüber treten könnte. In der „harten“ Version ist ein Nebenprodukt in jeder Hinsicht nutzlos. Seine Existenz ist so überflüssig, zugleich aber auch so zwangsläufig wie die des Bauchnabels. Man könnte ohne ihn leben, aber es geht ganz einfach nicht. Entsprechend dieser Sichtweise könnte man beispielsweise vermuten, dass unsere Hirnphysiologie soviel lebenswichtige Betriebsarbeit leistet, dass ästhetische Kognitionen gleichsam als unvermeidbare Nebenprodukte zufällig mit abfallen, zwar zu nichts nutze, aber eben auch nicht schädlich, jedenfalls nicht so schädlich, dass es sich für den Organismus lohnen würde, sie aufwendig zu verhindern. Dieses Bild ist vergleichbar mit einem Motor, der auch nutzlose Wärme abgibt, obwohl seine eigentliche Funktion in der Produktion von Antriebskraft liegt.

In der „weichen“ Version entstehen Nebenprodukte durch den Transfer von an sich funktionalen Merkmalen in andere Wirkzusammenhänge, d.h. wenn adaptive Merkmale außerhalb ihres funktionellen Entstehungskontexts in Lebensbereichen sichtbar werden, an die sie ursprünglich nicht angepasst sind. So könnte man vermuten, dass die Ästhetik der Kunstwelt als Nebenprodukt jener selektierten Ästhetik der Lebenswelt aufliegt, von der eingangs die Rede war. Naturschönes bevorzugen wir aus adaptiven Gründen nach bestimmten Kriterien, beispielsweise nach seinen Symmetrien und Proportionen. Diese Kriterien lassen sich aber auch in der Welt der Artefakte finden, und weil wir gar nicht anders können, als mit offenen Augen und ästhetischem Urteilswillen durchs Leben zu gehen, empfinden wir auch Dinge schön, um die es in der Evolution ästhetischer Präferenzen niemals gehen konnte. Die Schönheit eines symmetrisch gestalteten Faustkeils könnte danach vielleicht als nichtfunktionaler Ausfluss einer evolvierten und biologisch funktionalen Präferenz für beispielsweise symmetrische Gesichter verstanden werden, und die kunstvolle Präzision eines pas de deux aktiviert möglicherweise adaptive Präferenzen für Jugend und Vitalität. Und weil 
aus der Lust an der Erfüllung dieser Präferenzen eine kräftige Motivation erwächst, lernen wir kreativ mit den überkommenen Mechanismen der Ästhetik umzugehen. Wir machen Dinge schön, allein weil Schönheit schön ist - ohne je dafür biologisch belohnt zu werden. Kunst wäre in dieser Sicht so erfolgreich, weil sie uns unstillbar Schönheitssüchtigen vielversprechende Illusionen über eine vermeintlich attraktive Welt vorgaukelt. Das kunstästhetische Urteilsvermögen wäre also eine Transferleistung eines adaptiven naturästhetischen Urteilsvermögens - ohne aber selbst von irgendeiner Fitnessrelevanz zu sein. In dieser Sicht schießen die evolvierten Präferenzen über ihr eigentliches Ziel hinaus. Wir wären nicht nur wie ein Falter, der nächtens die Laterne umkreist, wir wären vielmehr wie ein Falter, dem es gelungen ist, Laternen zu erfinden, um sie voller Lust nächtens umkreisen zu können.

„Weiche“ Auffassungen von der Ästhetik als biologisches Nebenprodukt haben explizit oder implizit in der evolutionären Ästhetik bisher die dominierende Rolle gespielt. Eine ihrer Grundannahmen ist vielfach formuliert und untersucht: Ästhetisches Empfinden ist an die Wahrnehmung besonders konfigurierter Reizmuster gebunden, ${ }^{5}$ wobei gegebenenfalls die biologischen Ursprünge und die adaptive Logik dieser „Auslösemechanismen" in der Auseinandersetzung der Menschen mit der sie umgebenden Natur verortet werden. Das integrierte Ensemble dieser biologischen Auslösemechanismen bildet - der von Chomsky gefundenen linguistischen Tiefenstruktur vergleichbar - eine ästhetische Uni-

5 Aiken, N. E.: „Power through art“, in: Falger, V. S. E./Meyer, P./van der Dennen, J. M. G. (Hrsg.): Sociobiology and Politics, Stanford/London 1985, S. 215-228; Baukus, P.: „Biologie der ästhetischen Wahrnehmung“, in: Riedl, R./Delpos, M. (Hrsg.): Die Evolutionäre Erkenntnistheorie im Spiegel der Wissenschaften, Wien 1996, S. 239-261; Coss, R. G.: „The role of evolved perceptual biases in art and design“, in: Voland, E./Grammer. K. (Hrsg.): Evolutionary Aesthetics, Heidelberg 2003, S. 69-130; EiblEibesfeldt, I.: „The biological foundation of aesthetics“, in: Rentschler, I./Herzberger, B./Epstein, D. (Hrsg.): Beauty and the Brain - Biological Aspects of Aesthetics, Basel 1988, S. 29-68; Pinker, S.: How the Mind Works, New York 1999; Ralevski, E.: „Aesthetics and art from an evolutionary perspective", in: Evolution and Cognition, 6 (2000), S. 84103; Richter, K.: Die Herkunft des Schönen - Grundzüge der evolutionären Ästhetik, Mainz 1999; Sitte, P.: „Bioästhetik - Biologie zwischen Erkennen und Erleben“, in: Sitte, P. (Hrsg.): Jahrhundertwissenschaft Biologie - Die großen Themen, München 1999, S. 407-425; Sütterlin, C.: „From sign and schema to iconic representation. Evolutionary aesthetics of pictorial art", in: Voland, E./Grammer, K. (Hrsg.): Evolutionary Aesthetics, Heidelberg 2003, S. 131-170. 
versalgrammatik $^{6}$, jene Klaviatur, der sich das Schöne in seinen unterschiedlichsten und vielfältigsten, auch artifiziellen Manifestationen bedient. Als genetisch fixierte Universalie ist sie allen Menschen unabhängig von Kultur und Mode eigen, und wegen ihres ,angeborenen“ apriori-Charakters sorgt sie dafür, ,daß wir Menschen mit unserer ästhetischen Urteilskraft [...] mehr vermögen, als wir gelernt haben“7

Ein Vergleich mit einem anderen Nebenprodukt, nämlich der Erregbarkeit durch Pornographie, lässt allerdings Zweifel aufkommen, ob Kunstästhetik tatsächlich auf nichts weiter als eine einfache Transferleistung zurückgeht. Pornographie konnte in die Welt kommen - obwohl ihr Konsum nicht zu generativen Konsequenzen führt - weil sie sowohl auf sensorischen als auch emotionalen Aspekten der evolvierten Sexualität aufbaut. Abbildungen sexueller Stimuli finden denselben sensorischen Anklang wie die Stimuli selbst, und als Reaktion stellt sich (bei passender sexueller Orientierung) in beiden Fällen sexuelle Erregung ein. Sensorischer Appeal und emotionale Erregung sind in beiden Fällen (dem Nebenprodukt und der Angepasstheit) identisch. Mit der Ästhetik verhält es sich allerdings deutlich anders. Selbst wenn es stimmen sollte, dass das schön Gemachte aus evolvierten Reizkonfigurationen besteht und deshalb eine ,natürliche“ sensorische Attraktivität besitzt, bleibt die Frage nach der Vergleichbarkeit der emotionalen Reaktivität. Geraten wir beim Anblick eines symmetrischen Artefakts in eine sexuelle Appetenz, wie wir es vielleicht beim Anblick eines symmetrischen Gesichts tun? Finden wir bei Hunger die Stillleben flämischer Meister schöner? Oder bekommen wir gar bei ihrem Anblick Appetit? Wenngleich dies letztlich gar nicht völlig ausgeschlossen sein mag, ist es insgesamt aber eher unwahrscheinlich, dass mit der Wahrnehmung von Kunstschönem sich nach Art und Stärke dieselben emotionalen Erregungen einstellen wie bei der Wahrnehmung von Naturschönem. In der von Menschen gemachten Welt sind ästhetische Präferenzen offensichtlich mehr als pure Übertragungen archaischer Kompetenzen. Der Ästhetik natürlicher Merkmale, mit ihren typischen Spielwiesen der Erotik und Biophilie, ist eine Ästhetik gemachter Merkmale beigeordnet, zu deren Erklärung der Verweis auf evolvierte Nahrungs-, Habitat- und Partnerwahlpräferenzen nicht ausreicht.

Wären ästhetische Präferenzen in artifiziellen Kontexten lediglich funktionslose Nebenprodukte von ästhetischen Präferenzen in natürlichen Kontexten, wären sie Nebenprodukte geblieben, die bemerkens-

6 Richter (wie Anm. 5).

7 Richter (wie Anm. 5), S. 50. 
werterweise von keinem anderen Mechanismus ko-optiert wurden und an denen deshalb keine selektiven Prozesse ansetzen können. Aber wie wahrscheinlich wäre solch ein Fall? Jede evolutionäre Umkonstruktion muss zwangsläufig an dem aus früheren Evolutionsprozessen überkommenen Material ansetzen - an einem Material, das im Hinblick auf das spätere Merkmal nicht funktional gewesen ist. Biologische Angepasstheiten haben deshalb notwendigerweise eine Nebenproduktkomponente in ihrer frühesten Geschichte, sind aber gleichwohl durch die natürliche Selektion geformt und gestaltet worden. Die Fischflosse ist für das Leben im Wasser selektiert, gleichwohl spielte sie - wegen ihres Nebeneffekts, Gewichte tragen zu können - die Rolle einer Disposition bei der Evolution der Extremitäten der Landwirbeltiere. Nebenprodukte können am Anfang einer Merkmalsgeschichte stehen, aber wie wahrscheinlich ist es, dass sie keine adaptive Entwicklung auf den Weg bringen? Wie wahrscheinlich ist es, dass zufällig entstandene Nebenprodukte "für immer" Nebenprodukte geblieben sind, sie also von der natürlichen Selektion gleichsam „übersehen“ worden sein könnten? Bei solch komplexen (und teueren, weil viel Betriebsenergie verbrauchenden) Phänomenen wie ästhetischen Kognitionen jedenfalls erscheint es äußerst fragwürdig, dass sie sich von ihrer ursprünglichen Domäne, beispielsweise der Erotik, in eine andere, etwa in die der bildenden Kunst, haben transferieren lassen ohne dass damit ein Funktionsgewinn einhergegangen wäre. Wahrscheinlicher scheint, dass evolvierte biologische Präferenzen (etwa aus dem Bereich der sexuellen Ästhetik) mit ihrer Übertragung einen anderen Kontext (hier in die Welt der Artefakte) den Charakter von Prädispositionen für neuartige Entwicklungen annehmen und damit sehr schnell aus ihrer ursprünglichen Natur als Nebenprodukt herauswachsen. In dem $\mathrm{Maße}$, wie die natürlich selektierte ästhetische Präferenz in die Welt der Artefakte eindrang, wird sie von vielerlei sozialen Prozessen instrumentalisiert worden sein. Sie begann, eine neue signifikante Rolle zu spielen, womit wir es mit einer typischen Exaptation ${ }^{8}$ zu tun hätten.

Und schließlich: warum sollten Menschen mit gelegentlich immensem Aufwand Schönheit produzieren? Unter Annahme der Nebenprodukt-Hypothese wäre lediglich zu erwarten, dass wir spontan das Vorfindliche in unserer Lebenswelt - einschließlich der Artefakte - ästhetisch bewerten, nicht aber dass wir uns in nichtfunktionalen Aktivitäten verlieren. Weshalb sollten wir Kosten in Kauf nehmen, indem wir Zeit, Ressourcen und Risiken in das making special investieren, wenn sich

8 Gould, S. J.: „Exaptation: A crucial tool for an evolutionary psychology“, in: Journal of Social Issues, 47 (1991), S. 43-65. 
dieser Einsatz letztlich nicht auszahlt - ,auszahlt“ in der Währung, in der der Erfolg eines jeden biologischen Merkmals gemessen wird, nämlich in reproduktiver Fitness? Allein schon wegen der genannten Probleme mit der Nebenprodukt-Hypothese scheint in der evolutionären Ästhetik der alternative Blick einer adaptationistischen Perspektive gut begründet. Diese zu entwickeln ist Ziel der folgenden Abschnitte. ${ }^{9}$

\section{Nützlichkeits- und Signalevolution}

Im Zusammenhang von Sexualität und Erotik wird die Evolution von ästhetischen Präferenzen zunehmend besser verstanden. Was anzieht und erregt, enthält ein verlässliches Signal über die zu erwartenden Gewinne. Sexuelle Schönheit signalisiert, was Biologen good genes genannt haben. Damit ist die genotypisch determinierte Fähigkeit gemeint, mit pathogenem Stress und anderen Fährnissen des Lebens fertig zu werden. Good genes offenbaren sich phänotypisch in jenen Merkmalen, die - genau aus diesem Grund - in der sexuellen Ästhetik von herausragender Bedeutung sind, beispielsweise in der Gesichts- und Körpersymmetrie. ${ }^{10}$ Bei der Partnerwahl ästhetischen Standards zu folgen, bedeutet letztlich auf der Suche nach möglichst „fitten“ Partnern zu sein. Und weil Fitness erblich ist, ${ }^{11}$ kommt es automatisch zu einer evolutionären Kopplung von Indikator und Präferenz. Wer nach Schönheit strebt, wird im Mittel durch reproduktive Fitness belohnt und sorgt damit zugleich für die genetische Ausbreitung seiner/ihrer Präferenz. Das Schöne selbst ist nicht nützlich, es signalisiert nur Nützlichkeit. Der Pfauenschwanz ist nicht nützlich ganz im Gegenteil: er behindert beim Fliegen - wohl aber der Genotyp, der ihn am prächtigsten hervorgebracht hat: Die attraktivsten Pfauenhähne zeugen - weil im Besitz „guter Gene“ - die vitalsten Küken. ${ }^{12}$

Die Ausprägungsstärke der männlichen „Show-Merkmale“ kann selbstverständlich nur dann als zuverlässiger Indikator für „gute Gene“

9 Vgl. Voland, E.: „Aesthetic preferences in the world of artifacts - Adaptations for the evaluation of ,honest signals"?", in: Voland, E./Grammer, K. (Hrsg.): Evolutionary Aesthetics, Heidelberg 2003, S. 239-260.

10 Skamel, U.: „Beauty and sex appeal: Sexual selection of aesthetic preferences", in: Voland, E./Grammer, K. (Hrsg.): Evolutionary Aesthetics, Heidelberg 2003, S. 173-200; Thornhill, R./Gangestad, S. W.: „Facial attractiveness", in: Trends in Cognitive Sciences, 3 (1999), S. 452-460.

11 Møller, A. P./Alatalo, R. V.: „Good-genes effects in sexual selection“, in: Proceedings of the Royal Society London B, 266 (1999), S. 85-91.

12 Petrie, M.: „Improved growth and survival of offspring of peacocks with more elaborate trains“, in: Nature, 371 (1994), S. 598-599. 
dienen, wenn keine Täuschung möglich ist. Wäre sie möglich, d.h. könnten auch Männchen minderer genetischer „Tauglichkeit“ die jeweiligen Merkmale ebenfalls maximal ausbilden, würden sie im Interesse des eigenen Paarungserfolgs zweifellos geneigt sein, dies auch tatsächlich zu tun, also genetische Qualitäten nur vortäuschen. In der Folge würde die Korrelation zwischen Merkmalsausprägung und Fitness verschwinden, und die in Frage stehenden Merkmale verlören ihren Wert als Qualitätsmaßstab. Dass dies aber tatsächlich nicht der Fall ist, liegt an den Kosten dieser Merkmale. Ein attraktiver Pfauenschwanz, das kräftige Rot des Stichlings, der stramm aufrechte Hahnenkamm, der kräftezehrende Gesang der Nachtigall und all die anderen sexuell wirksamen Indikatoren „guter Gene“ können schlichtweg nicht billig nachgeahmt werden. Sie sind teuer in der Herstellung und/oder Unterhaltung und deshalb ehrliche Signale. Männchen, die ihre teuren Merkmale aufwendig zur Schau stellen, müssen sich dies notwendigerweise auch leisten können. Dieser $\mathrm{Zu}-$ sammenhang war Anlass für Zahavi, ${ }^{13}$ das „Handicap-Prinzip“ zu formulieren ${ }^{14}$ - häufig auch unter dem Namen ,costly signalling theory“ geführt. Die Logik fußt auf folgendem Zusammenhang: Eine gleiche $\mathrm{Zu}-$ nahme der Ausprägungsstärke eines Signals ist für weniger fitte relativ teurer als für fittere Individuen. Wer es sich leisten kann, teure Signale herzustellen, also Zusatzkosten in Kauf zu nehmen (sich selbst gleichsam zu handicappen), muss logischerweise wirklich fit sein, weshalb Weibchen ihre Partnerwahlpräferenzen an diesen nicht selten bizarr aufwendigen Merkmalen festmachen und so für deren evolutionäre Persistenz sorgen.

Weshalb diese Merkmale nicht billig nachgeahmt werden können, also weshalb sie eigentlich so teuer sind, bleibt in jedem Einzelfall zu hinterfragen. Es scheint so, dass bei männlichen Sexualmerkmalen häufig Testosteron eine wichtige Rolle spielt, denn dieses Sexualhormon führt einen physiologischen Doppeleffekt im Schlepptau: Es erleichtert

13 Zahavi, A.: „Mate selection - A selection for a handicap“, in: Journal of Theoretical Biology, 53 (1975), S. 205-214.

14 Grafen, A.: „Biological signals as handicaps“, in: Journal of Theoretical Biology, 144 (1990), S. 517-546; Iwasa, Y./Pomiankowski, A./Nee, S.: „The evolution of costly mate preferences II. The ,Handicap“ principle“, in: Evolution, 45 (1991), S. 1431-1442; Johnstone, R. A.: „Sexual selection, honest advertisement and the handicap principle: Reviewing the evidence“, in: Biological Review, 70 (1995), S. 1-65; Maynard Smith, J.: „Theories of sexual selection“,in: Trends in Ecology and Evolution,.6 (1991), S. 146151; Zahavi, A./Zahavi, A.: The Handicap Principle - A Missing Piece of Darwin's Puzzle, New York/Oxford 1997. 
zwar die Herstellung einiger der epigamen Merkmale, ${ }^{15}$ erhöht aber zugleich die Anfälligkeit gegen Parasiten, so dass nur gesunden Männchen, also solchen mit einer guten Immunkompetenz genug Testosteron zur Elaboration ihrer sexuellen Merkmale ,übrig bleibt“. Demnach wäre letztlich ein Abgleichproblem zwischen Investition in das Immunsystem und Investition in extravagante „Handicaps“ eine maßgebliche Bühne, auf der sich die Evolution von Partnerwahlpräferenzen abspielt.

Es sind aber nicht nur ,gute Gene“, auf die mit teuren auffälligen Signalen hingewiesen wird, sondern auch Qualitäten mit ausschließlich phänotypischen Benefits können öffentlich annonciert werden: politische Macht, materieller Reichtum, Kampfeskraft, Jagdgeschick etc., kurz: alles, was je nach vorherrschender Lebensweise zur ökonomischen und sozialen Wettbewerbsfähigkeit beiträgt und dessen öffentliche Zurschaustellung Prestige einfährt. ${ }^{16}$

Um die Evolution eines biologischen Merkmals zu verstehen, müssen wir zu aller erst zu klären versuchen, ob es sich um ein nützliches Merkmal handelt, also um ein Merkmal, mit dessen Hilfe Selbsterhaltung und Reproduktion besser gelingen, oder ob es sich vielmehr um ein Merkmal handelt, das ohne selbst direkt nützlich zu sein, nur in der Welt ist, weil es als ehrliches Signal verborgene Nützlichkeit anzeigt. Der Unterschied ist gravierend: Während die Nützlichkeitsselektion ökonomische Effizienz fördert, maximiert die Signalselektion kommunikative Reliabilität. $^{17}$ Effizienz wird erreicht, wenn mit einem Minimum an Investition ein Maximum an Nützlichkeit erreicht wird. Selektion von kommunikativer Verlässlichkeit führt demgegenüber $\mathrm{zu}$ dem, was auf den ersten Blick wie eine unökonomische Verschwendung knapper Ressourcen erscheint, nämlich zu vermeintlich funktionsloser Redundanz und Extravaganz der Signale - und dies nur deshalb, weil Signale teuer sein müssen, um überzeugen zu können. Für nützliche Merkmale sind ihre Herstellungskosten nachteilig, aber unvermeidbar. Bei Signalen kommt es demgegenüber gerade auf die Zusatzkosten an. Entgegen landläufiger ökonomischer Rationalität steigt die Nachfrage mit ihrem Preis. Nützliche Merkmale verlieren nicht an Nützlichkeit, wenn ihr Preis

15 Buchanan, K. L.: „Stress and the evolution of condition-dependent signals“, in: Trends in Ecology and Evolution, 15 (2000), S. 156-160; Owens, I. P. F./Short, R. V.: „Hormonal basis of sexual dimorphism in birds: Implication for new theories of sexual selection“, in: Trends in Ecology and Evolution, 10 (1995), S. 44-47.

16 Uhl, M./Voland, E.: Angeber haben mehr vom Leben, Heidelberg/Berlin 2002.

17 Zahavi/Zahavi (wie Anm. 14). 
sinkt. Signale hingegen verlieren ihre Funktion wenn ihre Herstellung inflationär billig wird.

Wenngleich Signale durch Signalselektion geformt werden, ist die Fähigkeit, diese Signale zu verstehen und angemessen darauf zu reagieren Ergebnis der Nützlichkeitsevolution. ${ }^{18}$ Ignorante Individuen werden im Durchschnitt biologisch weniger erfolgreich sein als jene, die die Zeichen zu deuten wissen und ihre sozialen Tendenzen von dem abhängig machen, was ihre Gegenüber über die Signale mitteilen.

In diesem Aufsatz geht es um die Frage, ob die Funktionslogik des Handicap-Prinzips nicht möglicherweise auch die Evolution artifizieller Ästhetik erklären könnte. Es greift damit eine Idee auf, die von Zahavi ${ }^{19}$ selbst angedacht wurde, ohne aber systematisch verfolgt worden zu sein. Vielleicht sind adaptive Interpretationen der artifiziellen Ästhetik bisher deshalb so unbefriedigend, weil sie nach Nützlichkeit gesucht haben, wo es keine gibt, und dabei zugleich den Signalcharakter des Schönen nicht angemessen beachtet haben. Könnte es sein, dass das artifiziell Schöne genauso wie das sexuell Schöne - nicht der Nützlichkeitsevolution unterliegt, sondern der Signalevolution? Ist Schönes schön, weil es teuer ist, und weil es in einer sozialen und zugleich kompetitiven Welt nützlich ist, Präferenzen für Personen zu entwickeln, die sich Schönes (also: Extrakosten) leisten können?

\section{Schönheit als Handicap}

Ein Zusammenhang von Kunst und Kommunikation wird zwar in vielen Theorien der Ästhetik, einschließlich der naturalistischen ${ }^{20}$ gesehen, allerdings häufig ohne der Frage nach der adaptiven Funktionslogik dieses Zusammenhangs ausreichend nachzugehen. Was genau wird zu welchem Zweck kommuniziert, und worin bestehen die selektiven Vorteile für Signalgeber und -empfänger, sich auf diesen künstlichen kommunikativen Austausch einzulassen? Wieso kann es fitnessförderlich ein, sich

18 Zahavi/Zahavi (wie Anm. 14).

19 Zahavi (wie Anm. 13).

20 Vgl. Aiken (wie Anm. 5); Coe, K.: „Art: The replicable unit - An inquiry into the possible origin of art as a social behavior", in: Journal of Social and Evolutionary Systems, 15 (1992), S. 217-234; Cooke, B./Turner, F. (Hrsg.): Biopoetics - Evolutionary Explorations in the Art, Lexington 1999; Dissanayake (wie Anm. 3); Eibl-Eibesfeldt (wie Anm. 5); Sütterlin, C.: „Art and indoctrination - From the Biblia pauperum to the Third Reich", in: Eibl-Eibesfeldt, I./Salter, F. (Hrsg.): Indoctrinability, Ideology and Warfare - Evolutionary Perspectives, New York/Oxford 1998, S. 279-300. 
mit aufwendigen, aber nutzlosen Signalen zu verständigen? Dies sind Fragen, wie sie eine Zahavische Perspektive der Ästhetik zu beantworten versucht. Wenn diese einigen Erklärungswert haben soll, wenn also das, was wir als schön betrachten, als Ergebnis der Signalevolution und unsere Präferenzen für das Schöne als Ergebnis der Nützlichkeitsevolution verstanden werden sollen, müssen drei Dinge zutreffen:

- Das Schöne muss als ehrliches Signal teuer sein.

- Das Schöne muss als ehrliches Signal für die Qualität des Signalgebers um die Aufmerksamkeit von bestimmten Adressaten buhlen, und

- es muss nützlich für diese Adressaten ein, den Signalgeber über das Schöne evaluieren zu können.

Um diese drei Hypothesen geht es in den folgenden Abschnitten.

\subsection{Hypothese 1: Making special bedeutet making expensive - Schönes ist teuer}

Die erste Hypothese fordert, dass das, was Dissanayake ${ }^{21}$ als making special bezeichnet hat, zugleich ein making expensive sein muss. Signale müssen ihrer Reliabilität wegen teuer sein. Erst wenn das making special einigen kostspieligen Aufwand erfordert, hat es eine Chance, das Attribut „schön“ verliehen zu bekommen. Was genau den Preis ausmacht, kann je nach Lebenssituation von Kultur zu Kultur, von Gruppe zu Gruppe sehr verschieden sein. Es muss nur, um in der Logik des Handicap-Prinzips als Kosten gelten zu können, sich in der Währung der natürlichen Selektion, der reproduktiven Fitness, bilanzieren lassen. Deshalb bestimmt investierter Lebensaufwand den Preis, und dessen Einsatz wiederum kann sich im Wesentlichen auf dreierlei Schienen abspielen: entweder in Form knapper oder riskant erworbener Güter, als Gesundheits- und Lebensrisiko und als Zeit, also als verlorene Opportunitäten für alternative Möglichkeiten der Fitnessmaximierung.

\subsubsection{Ressourcen}

Wer kennt nicht die kindliche Enttäuschung, wenn sich der schön glitzernde Edelstein am Strand nur als abgeschliffene Glasscherbe herausstellt? Dass wir den teueren Schmuck für den schöneren halten, dürfte eher die Regel als die Ausnahme sein. Und man denke an Kitsch, der al-

21 Dissanayake (wie Anm. 3). 
lein schon deshalb stigmatisiert ist, weil er Edles billig nachzuahmen versucht, während das monetär Teuere nur selten als Kitsch abgewertet wird. Der Zusammenhang von materieller Kostbarkeit und Schönheit ist kulturgeschichtlich vielfach auffindbar und ließe sich leicht durch weitere Fallbeispiele illuminieren. Für unser Thema ist nun die Kausalität der Korrelation zwischen Kostbarkeit und Schönheit von besonderem Interesse. Ist etwas schön, weil es kostbar ist, oder ist etwas kostbar, weil es schön ist?

In seiner „Theory of the Leisure Class“ hat Thorstein Veblen (ursprünglich: 1899) diesen Zusammenhang gründlich untersucht und resümiert:

Die höhere Befriedigung, die Gebrauch und Betrachtung teurer und angeblich schöner Dinge verschaffen, ist im allgemeinen nichts anderes als die Befriedigung unserer Vorliebe für das Kostspielige, dem wir die Maske der Schönheit umhängen. ${ }^{22}$

Und weiter heißt es:

Die Bedingung der Kostspieligkeit beeinflußt unseren Geschmack nämlich in der Art, daß sich der Geldwert mit der Schönheit unentwirrbar vermischt, worauf wir glauben, daß wir den Gegenstand nur um seiner Schönheit willen lieben. ${ }^{23}$

Es genügt also nicht, unseren sensorischen Apparat in besonderer Weise anzusprechen - das tut der Kitsch auch und das Hässliche allemal -, sondern es bedarf darüber hinaus der Kostspieligkeit, um als schön zu gelten. Es mag sein, dass Gold aus bestimmten neurophysiologischen Gründen sinnlich ansprechender ist als andere Metalle, sagen wir Aluminium, aber schön ist es wegen seines Wertes. Auch die blau-graue Tönung des Basalt mag sinnlich ansprechend sein, aber wer findet schon Rollsplitt ästhetisch reizvoll? Kurz: „Die Merkmale der Kostspieligkeit werden allmählich zu Merkmalen der Schönheit“ ‘. ${ }^{24}$

Nun könnte man kritisch einwenden, dass die Veblen'sche Sichtweise, die sich trotz ihrer akademisch und historisch so unterschiedlichen Herkunft doch verblüffend passgenau mit dem Zahavischen HandicapPrinzip trifft, möglicherweise für die entwickelten westlichen Agrar- und Industriegesellschaften mit ihrer Geldwirtschaft und ihren ausgeprägten Macht- und Besitzstratifikationen gelte, aber doch in den dazu sehr unterschiedenen sozio-ökologischen Milieus der Hominisation keine Ent-

22 Veblen, T.: Theorie der feinen Leute, Frankfurt a.M. 1986, S. 130.

23 Veblen (wie Anm. 22), S. 132.

24 Veblen (wie Anm. 22), S. 132. 
sprechung finden kann. Schließlich gelten die Wildbeutergesellschaften des Pleistozäns als sozial weitgehend egalitär, deren Subsistenzökonomie keine Ressourcenakkumulation oder gar Mehrwertproduktion zuließ. Trotz dieses Kontrasts galt aber - nach allem, was wir wissen - „schon immer", und das heißt seit Beginn der Kunstgeschichte von ca. 50.000 bis 60.000 Jahren, gerade das Kostbare und nicht etwa das Gewöhnliche als schön. Zu den historisch ältesten Formen des making special gehört die Körperbemalung, ${ }^{25}$ und dazu wurden ganz selbstverständlich edle Erden verwendet, die nur unter Aufwand besorgt werden konnten. $\mathrm{Coe}^{26}$ berichtet von australischen Aborigines, die Feindesland riskant durchqueren mussten, um an ihre Ocker zu gelangen, wie überhaupt die Ethnographie der rezenten Wildbeuter- und Pflanzergesellschaften vielfältige Belege dafür zusammengetragen hat, wie Kostbarkeit das Schöne konstituiert. ${ }^{27}$ Fazit: Teuer macht schön, und einer der Kostenfaktoren (oder: Schönheitsfaktoren, was in diesem Zusammenhang dasselbe ist) besteht in dem Ausmaß, wie knappe Güter verbraucht werden.

\subsubsection{Lebensrisiken (Vitalität)}

Schönheit konstituiert sich aber nicht nur über materielle Pracht. Signale können darüber hinaus auch deshalb teuer und somit ehrlich werden, weil $\mathrm{zu}$ ihrer Produktion notwendigerweise Gesundheit und Lebensrisiken eingesetzt werden müssen. In vielen historischen und traditionellen Gesellschaften gilt als schön, wessen Körper auf besondere Weise künstlich deformiert wurde, sei es durch Abfeilen der Zähne, durch Aufbringen von Narben und Tatoos, durch piercing oder durch Schädel-, Genitalund Fußdeformationen. Auch das aus der westlichen Modegeschichte bekannte Einschnüren der weiblichen Taille gehört in diese Kategorie. Alle diese somatischen Manifestationen des making special fordern selbstverständlich ihren mehr oder weniger hohen Preis an Vitalität. Schädeldeformationen reduzieren die Lebenstauglichkeit, Ablationen verursachen Schmerzen und Probleme beim Kauen und Sprechen, Vernarbung kann wegen der Infektionsrisiken tödlich enden, und der Hinweis, dass die eingeschnürte Wespentaille ungesund sein kann, findet sich bereits in

25 Watts, I.: „The origin of symbolic culture“, in: Dunbar, R./Knight, C./ Power, C. (Hrsg.): The Evolution of Culture - An Interdisciplinary View, Edinburgh 1999, S. 113-146.

26 Coe (wie Anm. 20).

27 Eibl-Eibesfeldt (wie Anm. 5); Power, C.: „,Beauty magic': The origins of art", in: Dunbar, R./Knight, C./Power, C. (Hrsg.): The Evolution of Culture - An Interdisciplinary View, Edinburgh 2000, S. 92-112. 
den ersten Ausgaben aufklärerischer Frauenmagazine. Die Verbannung des ungesunden Korsetts galt vielen geradezu als Symbol der Frauenemanzipation.

Zum Teil sind es die deformierten Menschen selbst, die Hand an sich legen lassen, zum Teil sind es Eltern, die gegebenenfalls gleich nach der Geburt mit der Umformung ihrer Kinder beginnen. Wenngleich die Pein, die damit verbunden ist, von den Kindern erlitten wird, sind es die Eltern, die gemäß der Logik des Handicap-Prinzips die Kosten dieses making special tragen. Schließlich verausgaben sie einen Teil ihrer reproduktiven Fitness, investieren also in der ultimativen Währung der Darwinischen Ökonomie. Die Formel lautet: Schön ist, was Vitalität verschwendet. Die Betonung liegt dabei auf dem Wort „verschwendet“, denn nicht gemeint sind all jene Narben, die ganz ungewollt aber zwangsläufig in den Fährnissen des Lebens entstehen. Ein durch Unfall verunstaltetes Gesicht wird wohl nur sehr selten als schön empfunden, schließlich legt es nicht Zeugnis von Verschwendung ab und hat deshalb nichts zu tun mit den Signalen, von denen das Handicap-Prinzip handelt. Anders der gewollte Schmiss - er galt in entsprechenden Zirkeln als Prestige trächtiges und deshalb „schönes Handicap“!

\subsubsection{Zeit (Opportunitäten)}

Und schließlich ist es Zeit, die den Preis bestimmt - Zeit, die eingesetzt werden muss, um einen Gegenstand kunstvoll herzurichten oder die man braucht, bestimmte Techniken zu lernen und zu vervollkommnen. Nur wer viel übt, kann schön musizieren, singen, schnitzen, bildhauern, malen, sticken, tanzen etc.. Weil aber nicht jeder, der lange übt, diese Techniken schließlich gut beherrscht, ist das Zeit/Leistungsverhältnis ein brauchbares Maß für das, was man gemeinhin „Begabung“ nennt und worin sich bestimmte Aspekte der Intelligenz und Kreativität ausdrücken. Zeit ist deshalb so kostbar, weil ihre Verschwendung Opportunitätskosten verursachen kann, denn schließlich könnte man, anstatt ausdauernd $\mathrm{zu}$ üben oder langwierig zu gestalten, produktiv arbeiten, Ressourcen anhäufen und diese - wie üblich - in Verpaarung und Reproduktion stecken. Wer dies jedoch sichtbar nicht tut, wer beispielsweise als steinzeitlicher Jäger nicht der Gazelle nachstellt, sondern stattdessen Flöte spielt, zahlt einen Preis, und weil nicht jeder aus puren Selbsterhaltungsgründen diesen Preis bezahlen kann, wird Zeitverschwendung zu einem ehrlichen Signal. „Demonstrativer Müßiggang“ nennt Thorstein Veblen in seiner „Theory of the Leisure Class“ diese Form der Investi- 
tion in soziales Prestige. Wer was gelten will, darf nicht produktiv sein, sonst würde er sich nicht von der Masse der armen Schlucker am Rande ihrer Existenz unterscheiden können. Wer was gelten will, muss stattdessen verschwenderisch mit knappen Dingen umgehen, eben auch mit Zeit.

Schön ist, was zu seiner Produktion wertvolle Zeit verbraucht, und deshalb gelten bei den Eipo aus den Hochtälern Neuguineas Tanznetze schön, die - zu nichts Praktischem nutze - sehr viel Zeit und geübte Fingerfertigkeit zu ihrer Herstellung erfordern und nur deshalb ihren Träger schmücken und ihm Prestige verleihen. ${ }^{28}$ Und deshalb galten den Schwälmer Bauern wie wohl allen anderen europäischen Landsmannschaften auch, kunstvolle Trachten als schön, die - ebenfalls ohne praktischen Nutzen - nur sehr mühsam herzurichten sind. Und aus demselben Grund gilt vermutlich vielen von uns handwerklich hergestellte Ware als schöner als dieselben Güter aus industrieller Produktion - und dies, obwohl Industrieware nicht selten perfekter vom Fließband rollt, als es die immer leicht unregelmäßig-fehlerhafte Handarbeit je sein kann. Es ist die Investition durch Extra-Arbeit, die diesen Dingen ästhetischen Wert verleiht. Nur was teuer ist, kann schön sein, und Zeit (genauer: Opportunitätskosten) ist eine der drei Preiskomponenten.

Wenn aber etwa wegen technischer Entwicklungen der Preis sinkt, d.h. wenn das making special keine nennenswerte Investition aus einem begrenzten persönlichen Zeitbudget mehr erfordert, verlieren die Dinge ihren ästhetischen Reiz. Schönheit unterliegt dann ganz unabhängig von ihrem sensorischen Appeal der inflationären Abwertung. Die Möglichkeit der technisch-automatischen Herstellung von Spitze hat das Interesse an ihr nahezu verschwinden lassen. Samt und Seide, früher Garanten eines schönen outfits, haben sich mehr oder weniger in die Reihe ,gewöhnlicher" Gewebe, Baumwolle unter ihnen, eingeordnet. Schnitzereien aus Oberammergau stehen heute unter „Maschinenverdacht“ und werden deshalb heutzutage im Unterschied zu früher eher als Kitsch denn als Kunst betrachtet. Es gibt Computerprogramme, die Musik im Stil alter Meister produzieren und zwar angeblich in einer Güte, die es selbst Experten schwer macht, die Neuproduktionen als solche zu erkennen. Dennoch - wir werden ganz irrational Vivaldi im Original schöner finden, als die stilistisch durchaus gleichwertigen Kunstprodukte der Computertechnik, denn unsere ästhetischen Algorithmen flüstern uns: Was billig herzustellen ist, kann nicht schön sein.

28 Eibl-Eibesfeldt (wie Anm. 5). 


\subsubsection{Fazit: Nur Teures wird als schön empfunden}

Nur Teures wird als schön empfunden. Dieser Satz gilt freilich nur unter Beachtung der verschiedenen Kostenfunktionen, zu denen neben den Ressourcenwerten auch investierte Zeit und Gesundheit gehören. Es mag überraschen, dass diese drei an sich sehr unterschiedlichen Währungen gemeinsam das Schöne konstituieren, wo sie sich doch sonst im Leben kaum zu berühren scheinen. Als unterschiedliche Formen des Lebensaufwands sind sie jedoch in eine gemeinsame Leitwährung konvertierbar, nämlich in ,reproduktive Fitness“. Das ist die Währung des Darwinischen Prinzips, auf die zu maximieren alle Organismen, einschließlich des Menschen, von Natur aus eingestellt sind. Zu diesem Zweck erschließen und akkumulieren sie Ressourcen, investieren in ihre Gesundheit und Selbsterhaltung und in die ihrer Nachkommen und gehen Kosten/Nutzen-orientiert mit Zeit um. Wenn immer aber Ressourcen, Vitalität und Zeit verausgabt werden, werden Lebens- und Reproduktionschancen abgeschöpft, und deshalb drückt diese ungleiche Trias den biologischen Merkmalen das ultimate Preisschild auf. Auch der Preis von Signalen bestimmt sich in letzter Analyse durch den Einsatz von reproduktiver Fitness, die zu ihrer Herstellung nötig waren - wenngleich sich die Kosten phänomenologisch vielfältig ausdrücken können.

Nebenbei: Dass sich hinter dem making special ein making expensive verbirgt, wird indirekt auch durch archäologische Evidenz gestützt, denn bereits die ersten gut nachweisbaren künstlerisch ambitionierten Ausdrucksformen der Menschen, die Faustkeile, und später die Figurinen aus dem zu Ende gehenden Mittelpaläolithikum und schließlich die Höhlenmalerein waren, was man getrost „Meisterwerke“ nennen kann. Die billige, einfältige Kunstproduktion - etwa in Form von Kritzeleien - hat ganz offensichtlich nicht am Beginn der Kunstgeschichte gestanden, ${ }^{29}$ sondern es ging allem Anschein nach von Anfang an um teure Signale.

Wer aber in der Lage ist, seinen Status fälschungssicher anzuzeigen, gewinnt gesellschaftliches Prestige, jenes knappe Gut, um das auf der psychologisch wahrgenommen Ebene unter Umständen mit enormen Einsatz konkurriert wird. Wer Prestige vermehren will, muss anzeigen, dass er es verdient, und dies kann er unmissverständlich durch Verschwendung - in der Diktion Veblens durch ,demonstrativen Konsum und Müßiggang“ - tun. Aus der soziobiologischen Perspektive Zahavis ist Verschwendung (und dazu gehört auch die sich nicht durch Nützlich-

29 Mithen, S.: „Handaxes: The first aesthetic artefacts“, in: Voland, E./Grammer, K. (Hrsg.): Evolutionary Aesthetics, Heidelberg 2003, S. 261-275. 
keit amortisierende Investition in das making special) ein ehrliches Signal, das es Betrügern, die kein Prestige verdienen, unmöglich macht, welches zu erschleichen. Hypothese 1 scheint vielfach belegt: Das making special ist seinem Wesen nach ein making expensive.

\subsection{Hypothese 2: Schönes signalisiert Qualität}

Als Signalgeber gilt, wer die Kosten des Signals trägt. Das kann natürlich zuallererst der Produzent des Signals selbst sein, also derjenige, der unter Einsatz seines Lebensaufwands das making special bewerkstelligt. Es kann aber auch eine Familiengruppe sein, die gemeinschaftlich in ein Signal investiert (die Totempfähle nordwestamerikanischer Indianer sind hierfür ein Beispiel). Und es kann auch ebenso derjenige sein, der als Sponsor oder Auftraggeber die Arbeitszeit und geübte Kunstfertigkeit geschickter und kreativer Menschen bezahlt, um Schönes produzieren zu lassen. In solchen Fällen wird man den handwerklich Tätigen gegebenenfalls nicht absprechen wollen, begnadete Künstler zu sein - im Erklärungszentrum des Handicap-Prinzips stehen sie allerdings nicht, weil sie den Preis des Signals nicht bezahlen, und dies umso weniger, je ökonomisch erfolgreicher sie ihre Begabung zu Markte tragen.

In einer Tauschgesellschaft, in der Lebensaufwand zur Handelsware wird und den Gesetzen von Angebot und Nachfrage unterliegt, muss es deshalb keinesfalls der Produzent des Schönen selbst sein, der die Kosten des Signals trägt. Maßgeblich im Sinne einer Darwinischen Ästhetik ist vielmehr, wer letztlich die Investition in das Schöne bezahlt. Deshalb ist die Prachtfassade zu aller erst ein Signal des Fürsten und möglicherweise erst nachgeordnet - und dann in einem viel engeren Kontext - auch ein Signal des Steinmetz. Der Park ist vorrangig in Signal des Schlossherrn, nicht des Gärtners, der Altar eines der Kirche, nicht des Holzschnitzers. In der Arbeit von Steinmetz, Gärtner und Holzschnitzer drückt sich selbstverständlich auch deren Qualifikation aus. Jedoch darf in diesen Fällen die Güte der Arbeit nicht als Signal im Sinne des Handicap-Prinzips missverstanden werden, denn gute Arbeit wird ökonomisch entlohnt und ist deshalb ganz unmittelbar „nützlich“. Die Arbeit wird ja nicht in ein teures, mit persönlichen Kosten verbundenes „Handicap“ investiert, sondern gegen Lebensvorteile eingetauscht. Fertigkeiten und Fähigkeiten best möglich ökonomisch zu verwerten ist nichts, was die evolutionäre Signaltheorie zu erklären vorgibt, denn hier steht der unmittelbare Gewinn im Vordergrund, nicht die teure und deutlich sichtbare Investition in offensichtlich Nutzloses. 
„Ehrliche Signale“ sind im Tierreich in dreierlei Kontexten evolviert, ${ }^{30}$ nämlich in der zwischenartlichen Kommunikation zwischen Beute und ihren Räubern; in der sozialen Konkurrenz um Rangpositionen, wo sie helfen, kampflos Hierarchien zu verhandeln, und schließlich in der sexuellen Konkurrenz, wo sie Rückschlüsse auf die „Partnerqualität" erlauben. Einmal abgesehen von der Beute/Räuber-Kommunikation, die beim Menschen aufgrund seiner ökologischen Dominanz keine nennenswerte Rolle mehr spielt, haben sich die beiden anderen Arenen der Signalevolution nahtlos in die menschliche Kulturgeschichte verlängert. Soziale Rangpositionen und Partnerqualität werden öffentlich annonciert und dies vor allem auch durch künstliche, aber nichtsdestoweniger notwendigerweise „ehrliche Signale“. Darüber hinaus kommt als typisch menschliche Errungenschaft ein Signalsystem hinzu, das moralische Tugendhaftigkeit anzeigt. ${ }^{31}$

Wenn die Hypothese stimmt, dass ästhetische Präferenzen als nützliche Decoder „ehrlicher Signale“ evolviert sind, müssen wir erwarten, dass das Kunstschöne als Medium fälschungssicherer Information entweder in der sexuellen Konkurrenz eine Rolle spielt oder auf den verschieden Bühnen des sozialen Wettbewerbs um Macht nach außen und Solidarität nach innen. Für alle drei Vermutungen gibt es gut untersuchte Belege.

\subsubsection{Schön und sexy}

Auf die Frage, warum sie eigentlich mühevoll Kleidung und Gebrauchsgegenstände dekorieren, antworteten die von Polly Wiessner ${ }^{32}$ interviewten Kalahari San ,that they want to present a positive image to partners in reciprocity and to members of the opposite sex" ${ }^{33}$ Hier findet sich wohl sehr viel Alltagswissen aus vermutlich allen Kulturen dieser Welt wieder. Wenn es darum geht, sich sexuell attraktiv in Szene zu setzen, spielt nicht nur die natürliche, sondern auch die artifizielle Schönheit eine wichtige Rolle.

30 Zahavi/Zahavi (wie Anm. 14).

31 Voland, E.: „Normentreue zwischen Reziprozität und Prestige-Ökonomie Soziobiologische Interpretationen kostspieliger sozialer Konformität“, in: Lütge, C./Vollmer, G. (Hrsg.): Fakten statt Normen? Zur Rolle einzelwissenschaftlicher Argumente in einer naturalistischen Ethik, Baden-Baden 2004, S. 177-189.

32 Wiessner, P.: „Style and social information in Kalahari San projectile points“, in: American Antiquity, 48 (1983), S. 253-276.

33 Wiessner (wie Anm. 33), S. 258. 
Was, sofern es um das persönliche outfit geht, wohl nicht ausführlicher belegt $\mathrm{zu}$ werden braucht, nämlich die Interpretation des schön Gemachten als Blickfang in der Arena des sexuellen Wettbewerbs, findet seine Entsprechung auch in anderen Spielarten des making special. Von der Poesie, der Musik, der bildenden Kunst behauptet Miller ${ }^{34}$ im direkten Anschluss an Darwin, ${ }^{35}$ dass sie einzig als Werbeträger männlicher Interessen evolviert sind. Mit dem schönen Lied, dem schönen Gedicht, dem schönen Bild kreieren (vorrangig) Männer courtship displays, mit denen sie um die Gunst des (meist) anderen Geschlechts buhlen. Für den Zusammenhang zwischen künstlerischem und sexuellem Erfolg dienen ihm Gewährsleute wie Jimi Hendrix, Pablo Picasso, Charlie Chaplin, Honoré de Balzac und andere, die als nimmersatte Liebhaber ihr künstlerisches Leben durchschritten haben. Und selbst der biedere Max Frisch bekommt - offensichtlich ungewollt - diesen Zusammenhang zu spüren, denn wie beklagt er sich als 64-jähriger in Montauk:

Ein Arrivierter könnte aussehen wie ein Walroß, die Frauen geben sich nicht nur mit ihm ab, sondern entfalten unverlangt ihren Charme fast ohne Reserve. Erst auf der Straße, anonym im Gedränge, empfinde ich mich wieder als Walroß ganz und gar. ${ }^{36}$

Nachdrücklicher als derartige Anekdoten, für die sich sicherlich auch Gegenbeispiele finden ließen, spricht zweifellos die auffällige Altersund Geschlechtsverteilung von Künstlern für Millers ${ }^{37}$ Interpretation der Kunst als sexuell evolvierte courtship displays. Bekanntlich produzieren Männer insgesamt deutlich mehr Kunst als Frauen, was angesichts geschlechtstypischer Sexualstrategien auch zu erwarten ist. Darüber hinaus werden die meisten Kunstwerke von jung erwachsenen Männern produziert, also genau dann, wenn ihre sexuelle Konkurrenz am größten ist.

In vielen traditionellen und modernen Gesellschaften bilden Initiationsriten die traditionelle Plattform für sexuelle Selbstdarstellung. Die persönlichen Kosten der Initiation können beträchtlich sein, u.a. auch deshalb, weil sie mit körperlichen, z.B. genitalen Verstümmelungen ein-

34 Miller, G. F.: „Sexual selection for cultural displays“, in: Dunbar, R./ Knight, C./Power, C. (Hrsg.): The Evolution of Culture - An Interdisciplinary View, Edinburgh 1999, S. 71-91; Miller, G.: „Evolution of human music through sexual selection", in: Wallin, N. L./Merker, B./Brown, S. (Hrsg.): The Origins of Music, Cambridge/London 2000, S. 329-360.

35 Darwin, C.: The Descent of Man and Selection in Relation to Sex, London 1871.

36 Frisch, Max: Montauk, Frankfurt a.M. 1975.

37 Miller 1999 (wie Anm. 34). 
hergehen. ${ }^{38}$ Die Höhe der Kosten wird nicht zuletzt durch die lokale Ökologie mitbestimmt. Körperliche Verstümmelungen sind gerade in Regionen mit ausgeprägtem pathogenem Stress teuer, weil risikoreich. Und in perfekter Entsprechung zum Handicap-Prinzip sind sie ausgerechnet in den tropischen Regionen mit überdurchschnittlichem Infektionsrisiko traditionell verbreitet. Ausgerechnet also wo sie am teuersten sind, tragen Narben zur Schönheit von (meistens) Frauen bei. ${ }^{39}$ Die Botschaft ist offensichtlich: Der Heilungsprozess erlaubt Rückschlüsse auf die Immunkompetenz der Frauen, und die ist in einer pathogen riskanten Lebenswelt einer der entscheidenden Fitnessfaktoren. Die Partnerqualität steigt mit der Abwehrkraft, und diese wird - fälschungssicher - durch künstliche Verletzungen angezeigt.

\subsubsection{Schön und stark}

In der Renaissance [...] war der beste Künstler beim mächtigsten Herrscher: Dürer war Hofmaler von Kaiser Maximilian; der zweitbeste, Cranach, war Hofmaler beim zweitmächtigsten, dem Kurfürsten von Sachsen; der drittbeste, Grünewald, war Hofmaler beim Drittmächtigsten, dem Fürstbischof von Mainz und Herzog von Brandenburg Albrecht; der viertbeste Künstler, Holbein, fand in Deutschland keinen adäquaten Platz. Er wurde Hofmaler des englischen Königs. ${ }^{40}$

Wenngleich Kunsthistoriker vielleicht debattieren mögen, ob diese Interpretation Baumanns in ihrer rigiden Strenge wirklich zutrifft, wird eines nicht bezweifelt werden können, nämlich der historisch regelmäßig anzutreffende Zusammenhang zwischen Macht und der Schönheit ihrer Insignien. ${ }^{41}$ Man wollte und will wohl immer und überall repräsentieren, und dies kann angesichts einer steten Konkurrenz um die privilegierten Positionen einer Gesellschaft nur im Wettbewerb mit fälschungssicheren Signalen geschehen. Nur wer sich die besten Künstler leistet, dem glaubt

38 Low, B. S.: „Pathogen stress and polygyny in humans“, in: Betzig, L./Borgerhoff Mulder, M./Turke, P. (Hrsg.): Human Reproductive Behaviour - A Darwinian Perspective, Cambridge 1988, S. 115-127; Rowanchilde, R.: „Male genital modification - A sexual selection interpretation“, in: Human Nature, 7 (1996), S. 189-215; Power (wie Anm. 27).

39 Singh, D./Bronstad, P. M.: „Sex differences in the anatomical locations of human body scarification and tattooing as a function of pathogen prevalence“, in: Evolution and Human Behavior, 18 (1997), S. 403-416.

40 Baumann, C.: „Naturwissenschaft und Kunst - Versuche der Begegnung“, in: Nova acta Leopoldina NF, 77 (1998), S. 193-204, S. 200.

41 Sütterlin (wie Anm. 20). 
man, dass er reich (oder mächtig, meistens aber beides zugleich) ist. Weil demonstrative Verschwendung in diesen Kontexten Wettbewerbstauglichkeit in Machtsystemen annonciert (vergl. Neiman ${ }^{42}$ für eine Fallstudie zur Maya-Architektur), setzt sie ganz automatisch eine Spirale des ästhetischen Wettrüstens in Gang: Der Mächtigste muss immer etwas mehr in seine Statussignale investieren, also immer etwas mehr Schönheit produzieren lassen, als der Zweitmächtigste in der Lage ist.

Die Botschaft dieses Angebens ist offensichtlich. Wer Teures produzieren lässt, muss Menschen und Ressourcen kontrollieren können und ist deshalb einflussreich und mächtig. Die Rangliste der Investitionen in Repräsentation entspricht direkt der Rangliste an Einfluss und Macht. Die Logik dieses Signalsystems funktioniert gleichermaßen, ob Einzelpersonen, Dynastien, Clans, Stämme, Parteien, Staaten, Kirchen oder Konzerne im Wettbewerb stehen. Schönes entsteht folgerichtig umso wahrscheinlicher, je prävalenter Konkurrenz ist. Deshalb ist es sicherlich kein Zufall, wenn Kunsthistoriker in der deutschen Kleinstaaterei mit entsprechender Dichte wetteifernder Oberhäupter den Grund sehen, weshalb sich hier das Musikleben vielfältiger gestaltet hat als etwa im benachbarten zentralistischen Frankreich. Und die schönsten Bankenhochhäuser werden dort gebaut, wo schon die der Konkurrenz stehen. Nur der Beweis von Macht über Menschen und Reichtum gibt als ehrliches Signal einem Machtanspruch eine öffentlich anerkannte Berechtigung.

\subsubsection{Schön und moralisch gut}

Die frühe Menschheitsgeschichte war geprägt durch einen ständigen Wettbewerb autonomer Verwandtschafts-Gruppen um ökologische Lebensvorteile. $^{43}$ Evolutionärer Ausfluss dieser Zwischengruppenkonkurrenz war eine gefestigte, in group-/out group-Moral', deren wesentlichste Funktion darin bestand, die Angehörigen einer Gruppe zu einer sozialen Allianz zusammenzubinden und sie auf ein „Wir-Gefühl“ zu verpflichten. Aber wie alle öffentlichen Güter unterliegt auch die Gruppensolidarität dem ,Schwarzfahrer-Problem“. In einem Konflikt zwischen dem Eigeninteresse und dem Gruppenwohl siegt mit größerer

42 Neiman, F. D.: „Conspicuous consumption as wasteful advertising: A darwinian perspective on spatial patterns in classic Maya terminal monument dates", in: Barton, C. M./Clark, G. A. (Hrsg.): Rediscovering Darwin: Evolutionary Theory and Archeological Explanation, Arlington 1997, S. 267-290.

43 Alexander, R.: The Biology of Moral Systems, Hawthorne 1987. 
Wahrscheinlichkeit das Eigeninteresse. Man mag zwar geneigt sein, die Vorteile der Gruppenzugehörigkeit für sich persönlich best möglich zu nutzen, aber anderseits gibt es starke Anreize, als persönlicher Nutzenmaximierer möglichst Kosten zu vermeiden, die aus der sozialen Allianz erwachsen. Gruppensolidarität läuft deshalb immer Gefahr ausgebeutet zu werden - es sei denn, ihre Mitglieder und vor allem die neu Hinzukommenden bekunden mit „ehrlichen Signalen“ ihre moralische Integrität. ${ }^{44}$ Diese Funktion übernehmen Rituale. ${ }^{45}$ Wer bereit ist, die hohen Kosten eines Initiationsritus auf sich zu nehmen, bekennt sich für alle wahrnehmbar zu seiner in group und demonstriert die geforderte Loyalität. Die hohen Kosten der Initiation verhindern ein opportunistisches Schwarzfahren, denn Nettovorteile der Gruppenzugehörigkeit stellen sich erst ein, wenn die anfänglichen Eintrittskosten kompensiert sind. Außerdem versperren dauerhaft äußerlich sichtbare Zeichen ihrem Träger die Möglichkeit des opportunistisch-taktischen Gruppenwechsels und begründen so eine hohe Wahrscheinlichkeit für ein lebenslanges committment des Initianden. Er zeigt mit dem teuren Signal ein glaubwürdiges Interesse an der Zugehörigkeit zu dieser Gruppe. Er kauft Prestige und empfiehlt sich so als verlässlicher und moralisch guter Partner. ${ }^{46}$

So kommt es zu einer evolutionären Kopplung von „schön“ und „moralisch gut“, die nicht zuletzt in der westlichen Kulturgeschichte tiefe Spuren hinterlassen hat. Erst in der jüngsten Vergangenheit beginnt sich die aufgeklärte Einsicht durchzusetzen, dass äußere Schönheit und ,innere Werte" getrennt zu beobachten sind. Von der Antike bis zur Neuzeit war die Kopplung des moralischen mit dem ästhetischen Urteil common sense. „Das Gute ist dem Schönen inhärent, weil das Schöne dasselbe Substrat hat wie das Gute" heißt es bei Albertus Magnus ${ }^{47}$ stellvertretend für eine diesbezüglich wenig wechselvolle abendländische Ideengeschichte. Und natürlich sind die guten Helden unserer Geschichten schön und die bösen hässlich - in Groschenromanen ebenso wie in HollywoodProduktionen. Die Tendenz zur Vermengung ästhetischer und morali-

44 Dunbar, R.: „Culture, honesty and the freerider problem“, in: Dunbar, R./ Knight, C./Power, C. (Hrsg.): The Evolution of Culture - An Interdisciplinary View, Edinburgh 1999, S. 194-213.

45 Knight, C.: „Ritual/speech coevolution: A solution to the problem of deception“, in: Hurford, J.R./Studdert-Kennedy, M./Knight, C. (Hrsg.): Approaches to the Evolution of Language - Social and Cognitive Bases, Cambridge 1998, S. 68-91.

46 Knight (wie Anm. 44); Power (wie Anm. 5).

47 zitiert nach Henss, R.: ,, Spieglein, Spieglein an der Wand... “- Geschlecht, Alter und psychische Attraktivität, Weinheim 1992; S. 86. 
scher Urteile ist offensichtlich sehr tief in unseren emotionalen und kognitiven Mechanismen verankert, ${ }^{48}$ und es sieht ganz danach aus, dass es für diese Kopplung einen evolutionär nachvollziehbaren Hintergrund gibt.

\subsubsection{Fazit: Making special entstammt dem Wettbewerb um sexuelle, politische oder moralische Anerkennung}

Das Schöne hat Signalfunktion. Es kommuniziert soziobiologische Qualitäten desjenigen, der in die Produktion des Schönen investiert hat, wobei wir es mit einer Trias von Botschaften zu tun haben, die auf den ersten Blick wenig miteinander zu tun zu haben scheinen. Das „Ich bin fit“ entstammt der Sexualität, das „Ich bin stark“ der Konkurrenz um Macht und das „Ich bin gut“ der Moralität. Das gemeinsame dieser Botschaften besteht darin, dass ihre Kernaussagen nicht so ohne weiteres erkennbar sind. „Gute Gene“ kann mit nicht sehen, Macht und Stärke der politischen Protagonisten nur gelegentlich - und dann eventuell unter hohen persönlichen Risiken - wahrnehmen. Und moralische Integrität kann man nicht nur nicht sehen, sondern sie ist in einer Welt persönlicher Nutzenmaximierer a priori sogar unglaubwürdig. Alle drei Botschaften bedürfen also des Beweises ihres Wahrheitsgehalts und den liefern teure Signale, die allein aufgrund ihrer bloßen Existenz versteckte Qualitäten des Signalgebers belegen.

Signalgebern bleibt gar nichts anderes übrig, als sich auf den für sie so teuren Wettbewerb einzulassen, denn schließlich buhlen sie um Gefolgsleute, und die wollen überzeugt sein. Das verschwenderische $m a-$ king special dokumentiert öffentlich den eigenen Marktwert als Sexualund Sozialpartner. Vordergründig geht es um Aufmerksamkeit, letztlich aber um Anerkennung in den kritischen Augen potenzieller Partner womit wir bei der dritten Hypothese angelangt sind.

\subsection{Hypothese 3: Ästhetische Präferenzen sind nützlich}

Signalgeber haben ein persönliches Interesse daran, ein Publikum zu erreichen, dem sie ihre versteckten Qualitäten vorzeigen können. Allerdings sind die Interessen von Signalgeber und -empfänger nicht deckungsgleich. Kommunikation ist bekanntlich nicht evolviert, um „Tatsachen" auszutauschen, sondern wegen der Vorteile, die für Signalgeber

48 Henss (wie Anm. 46). 
aus der Beeinflussung des Verhaltens von Signalempfängern erwachsen. ${ }^{49}$ Einfache, ökonomisch knappe Embleme mit Botschaften von der Art „Ich bin fit, stark und moralisch gut“ konnten nicht evolvieren, weil eine naive, ungeprüfte Übernahme von sozial motivierter Information für die Signalempfänger hochgradig riskant wäre. Schließlich müssten sie damit rechnen, ausgebeutet zu werden. Andererseits haben aber auch die Signalempfänger ein vitales Interesse daran, über die versteckten Qualitäten ihrer Sozialpartner aufgeklärt zu sein, denn auch sie suchen best mögliche Sexualpartner, höchst potente Machiavellisten und möglichst verlässliche Kooperationspartner. Für Signalempfänger ist deshalb ein Detektor vorteilhaft, der die persönlichen Verlautbarungen der Signalgeber auf ihren „Wahrheitsgehalt“ hin überprüft und sie (die Signalgeber) nach ihrer Nützlichkeit als Partner in sexuellen oder sozialen Kontexten bewertet. Und genau diese Funktion erfüllt das ästhetische Urteil. Es bewertet „ehrliche Signale“. Ästhetische Präferenzen sind auch in der Welt der Artefakte das, was sind in der Welt natürlicher Merkmale immer schon waren: Orientierungshilfen bei wichtigen Lebensentscheidungen.

In den Selektionsprozessen während der Hominisation sind zweifellos jene Individuen belohnt worden, die in einer Welt sexueller und machiavellischer Konkurrenz die ,richtigen“ Partner-Entscheidungen getroffen haben. Das sind diejenigen gewesen, die sich nicht indiskriminativ verpaart haben, sondern den „Wert“ möglicher Partner einzuschätzen lernten, diejenigen, die sich nicht beliebigen Koalitionen angeschlossen haben, sondern Macht und Einfluss der politischen Figuren erkennen konnten und diejenigen, die sich nicht indiskriminativ solidarisch-altruistisch verhielten, sondern die moralische Verlässlichkeit möglicher Allianzpartner zu prüfen verstanden. Dies war ihnen nur deshalb möglich, weil Anbieter über teure und deshalb ehrliche Signale ihre jeweiligen Qualitäten als mögliche Sexual- und Sozialpartner mitgeteilt haben. Die psychobiologischen Steuerungsmechanismen sozialer Affekte verarbeiten diese Information und sorgen auf dieser Grundlage für wichtige Entscheidungen in einer kompetitiven Welt: Wem soll man sich hingezogen fühlen - sexuell, politisch, moralisch - und mit welcher Intensität? Das ästhetische Empfinden ist seinem Wesen nach ein Evaluationsvorgang und das ästhetische Urteil in seinem Kern ein Urteil über die soziobiologische Qualität derjenigen, die ehrliche Signale produzieren oder spon-

49 Dawkins, R./Krebs, J. R.: „Animal signals: Information or manipulation?“, in: Krebs, J./Davies, N. B. (Hrsg.): Behavioural Ecology: An Evolutionary Approach, Oxford 1978, S. 282-309. 
sern und damit um Partner für sexuelle, politische, oder moralische Kooperation werben.

\section{5. Über Geschmack lässt sich (nicht) streiten - Wieso eigentlich?}

Die soziobiologische Perspektive der Ästhetik nimmt gleichsam beiläufig und ganz unintentional Stellung zu der uralten philosophischen Debatte um die Ursprünge des Schönen. Liegen sie in den Dingen selbst oder in den Köpfen der Betrachter? Ist Schönheit eine Kategorie der Objekte oder der erkennenden Subjekte? In der fast zweieinhalbtausendjährigen philosophischen Debatte treffen realistische Positionen, die Schönheit als objektiv in der Realität vorgegeben betrachten, absolut unversöhnlich auf idealistische Positionen, die - in ihren härtesten Versionen - ästhetisches Empfinden als ausschließlich subjektive Leistung interpretieren, nicht begründbar, nicht objektivierbar, nicht einmal kommunizierbar. Es sollte klar geworden sein, dass keine der beiden Positionen in der soziobiologischen Sicht Unterstützung findet. Selbstverständlich haftet in gewisser Weise Schönheit den Dingen an. Signale sind reale, objektive, wahrnehmbare Fakten, und das, was ihre Schönheit konstituiert, ist bestimmbar (zur Erinnerung: es ist Höhe der Investition an Lebensaufwand). Aber genauso selbstverständlich ist das ästhetische Urteil Ergebnis eines subjektiven Evaluationsvorgangs der empirischen Faktenlage. Dasselbe Signal kann sehr unterschiedlich bewertet werden, denn Menschen sind Konstruktivisten. Sie bewerten das Wahrgenommene nach persönlichen Kriterien (wie denn auch sonst?) ohne aber - und das unterscheidet den Naturalismus der Soziobiologie von dem postmodernen anything goes sich in einer rational nicht zugänglichen Beliebigkeit zu verlieren. Das ästhetische Urteil ist eine subjektive Leistung einer objektiven artspezifischen Angepasstheit. „Beauty is in the eye of the beholder“ ist deshalb nur die halbe Wahrheit. Richtiger hat es Symons ${ }^{50}$ ausgedrückt: „Beauty is in the adaptations of the beholder". Angepasstheiten aber sind informationsverarbeitende Mechanismen zur Lösung biologischer Lebensund Reproduktionsprobleme, die natürlich sehr wohl subjektive Daten mitverarbeiten und deshalb bei gleichem input variablen output erzeugen können. Es gibt eben - aus Gründen biologischer Individualität - ver-

50 Symons, D.: „Beauty is in the adaptations of the beholder: The evolutionary psychology of human female sexual attractiveness", in: Abramson, P. R./Pinkerton, S. D. (Hrsg.): Sexual Nature - Sexual Culture, Chicago/London 1995, S. 80-118. 
schiedene Lebensprobleme mit unterschiedlichen Entscheidungsprozessen und deshalb auch verschiedene Geschmäcker, obwohl der Darwinische Algorithmus des ästhetischen Urteils als biologisch evolvierte artspezifische Universalie gedacht werden muss. 
MANFRED FAHLE

\section{ÄSTHETIK ALS TEILASPEKT BEI DER SYNTHESE MENSCHLICHER WAHRNEHMUNG}

Für Werner Maihofer

\section{Wahrnehmung, Ästhetik und das Hirn}

Im vorliegenden Text soll es um die Synthese der Wahrnehmung und um die Beziehung dieser synthetisierten Wahrnehmung zur Ästhetik gehen. Wahrnehmung ist, ebenso wie das Erleben ästhetischer Erfahrungen, ganz sicher eine Leistung, die ganz wesentlich auf der Funktion unseres zentralen Nervensystems, genauer: der Großhirnrinde, beruht. Lassen Sie mich daher damit beginnen, einige relevant erscheinende Aspekte der Hirnfunktion zu diskutieren.

Vor zehn Jahren war es noch stärker als heute en vogue, das Gehirn mittels einer Analogie zum Computer zu beschreiben. Mittlerweile hat sich aber zunehmend die Erkenntnis durchgesetzt, dass es sich beim menschlichen Gehirn keinesfalls um eine Art von Computer handelt. In unserem Kopf befindet sich keine Hardware, sondern eher ,wetware“. „Wetware“ bedeutet, dass die Bestandteile dieses als Gehirn bezeichneten informationsverarbeitenden Apparates überwiegend aus Wasser bestehen, also im Gegensatz zu den Chips der Computer ziemlich nass sind. Insofern kann die biologische ,wetware“ der ingenieurserzeugten ,hard-“ und ,software“ gegenüber gestellt werden. Diese „,wetware“ unseres Gehirnes folgt ganz eigenen Regeln, die sich grundlegend von den in Computern normalerweise verwendeten unterscheiden. Daher taugt die Computeranalogie nicht, wenn wir Hirnfunktionen in ihrem Verhältnis zu Wahrnehmung und Ästhetik verstehen wollen.

Wahrnehmung bedarf vermutlich keiner Definition, die umgangssprachliche Bedeutung ist ausreichend klar. Doch der zweite zentrale Begriff des Titels dieses Textes erfordert eine Definition. Laut Duden- 
Fremdwörterlexikon bezeichnet Ästhetik ,die Wissenschaft vom Schönen“ bzw. die „Lehre von der Gesetzmäßigkeit und Harmonie in Natur und Kunst". Ein verwandter Begriff, die Ästhesiologie, verbindet auf das Schönste die Wahrnehmung mit der Ästhetik. Er bezeichnet die „Lehre von den Sinnesorganen und ihren Funktionen“. Im Rahmen der Siegener Tagung haben wir Definitionen gehört von „Beauty is in the eye of the beholder“ über „Beauty is in the genome of the beholder“ bis zu „Meaning is in the eye of the decoder". Ich möchte eine weitere Definition anschließen, nämlich „Beauty is in the brain of the beholder“. Ich hoffe, Sie davon überzeugen zu können, dass diese Definition „Sinn“ macht.

\section{Individualität des visuellen Systems}

Wir beginnen also, wie oben angekündigt, damit, den Wahrnehmungsapparat zu analysieren. All unser Sehen startet in der Netzhaut mit Stäbchen und Zapfen, die das von der Außenwelt stammende Licht in elektrische Aktivität umwandeln. Das bedeutet, bereits in der Netzhaut findet ein Bruch der physikalischen Signal-Natur statt. Aus elektromagnetischen Wellen werden elektrische Impulse, die mit den Objekten, von denen diese elektromagnetischen Wellen ausgehen, keinesfalls identisch sind. Die Netzhaut ist übrigens „verdreht“, d.h. die Fotorezeptoren verstecken sich vor dem Licht und sind in die falsche Richtung ausgerichtet, d.h. vom Licht weg, in Richtung auf die Außenhülle des Auges. Kein Ingenieur würde eine solche Lösung wählen. Insofern ist diese Tatsache bereits ein Hinweis darauf, dass die Evolution einen gewissen Einfluss auf die Ausstattung unserer Sinnessysteme ausgeübt hat: die realisierten Problemlösungen wurden nicht am Reißbrett entworfen, sondern bauten auf bereits vorhandenen, entwicklungsgeschichtlich älteren, Nervensystemen auf.

Die Verteilung der verschiedenen Fotorezeptor-Klassen, und damit der verschiedenen Zapfen eines Individuums, kann seit einigen Jahren direkt sichtbar gemacht werden. Sie wissen alle, unsere Netzhaut besitzt drei verschiedene Fotorezeptoren-Typen für das Tagsehen, die in erster Näherung bevorzugt auf rotes Licht, grünes Licht und blaues Licht antworten. (Selbstverständlich ist das Licht eigentlich nicht farbig, sondern besitzt eine Wellenlänge - die Farbe entsteht erst im Gehirn). Eine Methode, die aus der Astronomie entliehen wurde, hat es ermöglicht, unter experimentellen Bedingungen das Auflösungsvermögen der Optik des Auges vorübergehend um den Faktor drei bis vier zu steigern. Das bedeutet, dass man durch die Optik des Auges auf die Fotorezeptoren 
schauen, die einzelnen Fotorezeptoren unterscheiden und diese selektiv bleichen kann. Man „überschüttet“ also die Netzhaut mit „rotem“ Licht, um die Fotorezeptoren, die darauf am stärksten reagieren, auszubleichen. Das Ausmaß der Bleichung jedes einzelnen Rezeptors kann mit Hilfe der neuen Methode gemessen werden. Dann verwendet man ,grünes“ Licht, um die Rezeptoren zu bleichen, die am stärksten auf diese Wellenlänge reagieren und danach jene, die auf „Blau“ reagieren. Auf diese Art und Weise konnte man bei mehreren Versuchspersonen die Verteilung der einzelnen Rezeptortypen untersuchen. Dabei stellte man zur großen Verwunderung fest, dass die Relation zwischen Grün- und RotRezeptoren bereits bei diesen wenigen Versuchspersonen um den Faktor zwei schwankte. Das heißt, eine der Versuchspersonen besaß relativ betrachtet doppelt so viele Rot-Rezeptoren wie die andere. Offenbar ist bereits auf der Ebene der Netzhaut Ihr „Rot“ etwas Anderes als mein „Rot“. Wenn wir beide auf das gleiche Objekt schauen, unterscheiden sich die Erregungen unserer Nervensysteme bereits auf der Ebene der Netzhäute. Mit anderen Worten: Individualität der Wahrnehmung manifestiert sich bereits auf der Ebene der Netzhaut.

\section{Einige allgemeine Prinzipien der Hirnfunktion: Spezialisierung}

Diese Individualisierung ist selbstverständlich nicht auf die Netzhaut beschränkt, sondern setzt sich im Gehirn fort. Die Repräsentation der Außenwelt im Gehirn lässt sich durch einige wenige allgemeine Prinzipien recht gut beschreiben. Eines dieser Prinzipien ist die Spezialisierung. Spezialisierung bedeutet, dass verschiedene Teile des Gehirns unterschiedliche Aufgaben erfüllen. Der hintere (okzipitale) Teil sowie Anteile des Scheitel- und Schläfenlappens (vgl. Abb. 1) vermitteln den Seheindruck. Im Schläfenlappen ist auch das Hirnzentrum für die Repräsentation des Raumes lokalisiert, im Scheitellappen die Körperfühlsphäre und im Frontallappen die Motorik sowie die „Persönlichkeit“. Vielen wird vielleicht noch aus dem Schulunterricht geläufig sein, wo die Körperfühlsphäre lokalisiert ist (Abb. 2). Unterschiedliche Teile des Cortex informieren uns darüber, wo wir berührt werden (sensorischer Homunculus) oder steuern die Bewegungen des Körpers (motorischer Homunculus). Diese Cortexareale sind recht gut geordnet, beispielsweise nimmt die Repräsentation des Gesichtes nur einen kleinen umschriebenen Teil des Cortex ein. Ein benachbarter Teil des Gehirns repräsentiert den Kopf, andere die Hände oder Füße. Direkt neben bzw. hinter dem motorischen 
Homunculus findet sich der sensorische Homunculus. Die Teile des Gehirns im braun eingefärbten Bereich repräsentieren verschiedene Teile des Körpers. Sie werden in Abb. 2 sehen, dass gute Entsprechungen zwischen der Motorik und der Sensorik existieren, mit einer kleinen Ausnahme: Ein bestimmter Teil des Körpers ist nur in der sensorischen Karte vorhanden, nicht aber in der motorischen Karte.

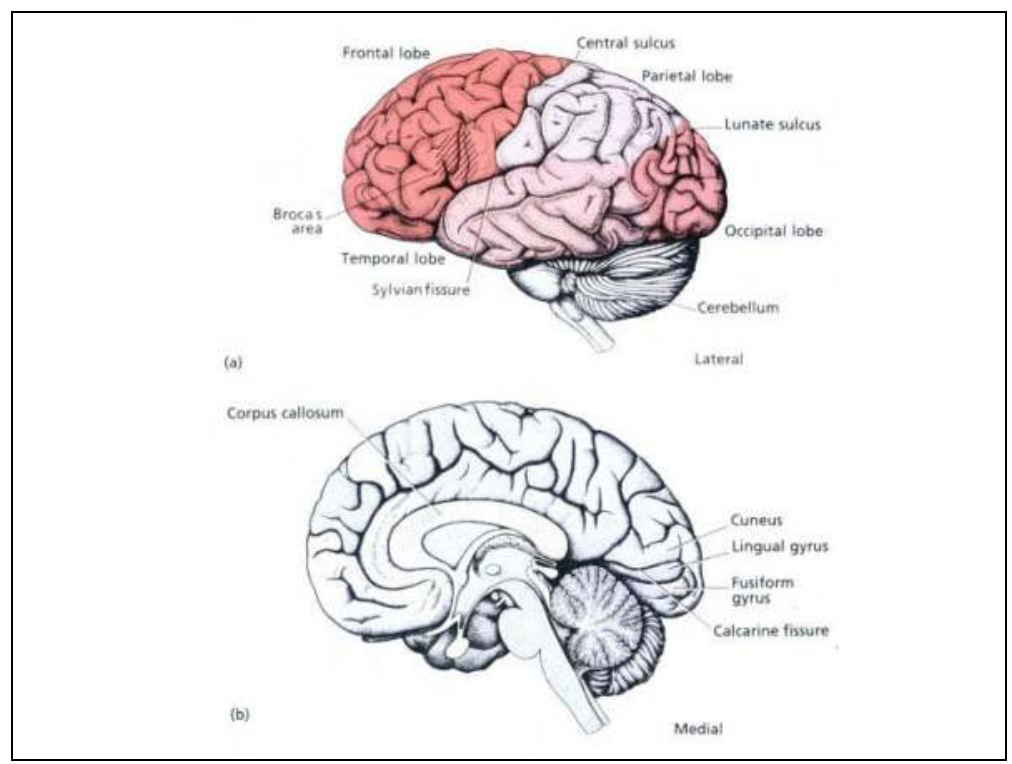

Abb. 1: Schematische Ansicht des menschlichen Gehirns mit makroskopischer Gliederung ${ }^{1}$

1 Zeki, S.: A Vision of the Brain, Oxford 1993, S. 18, Abb. 2.2. 


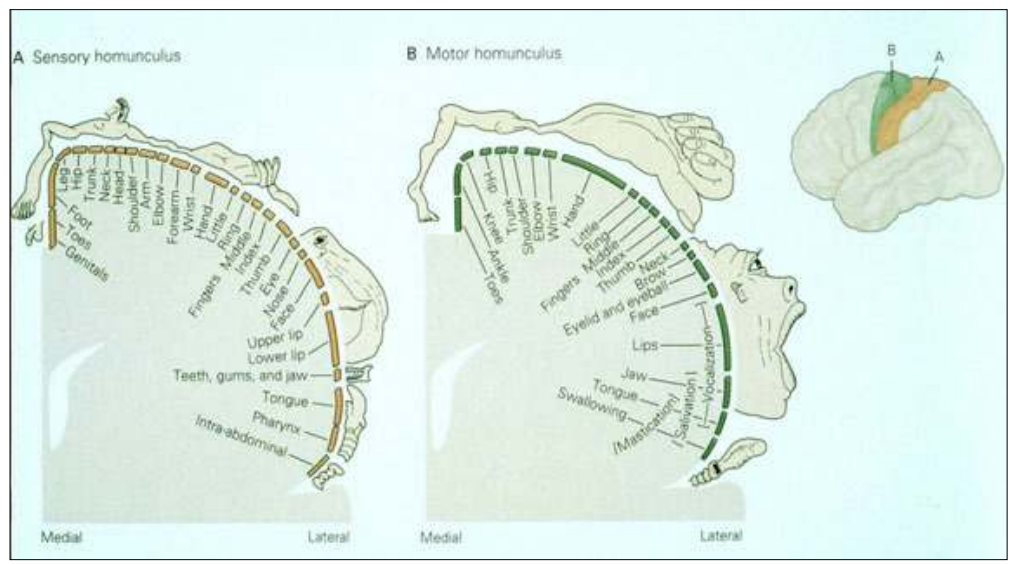

Abb. 2: Repräsentation des Körpers in der menschlichen Großhirnrinde (sensorischer Homunculus) sowie der ,Körpersteuerung “ (motorischer Homunculus) ${ }^{2}$

Die Ausdehnung unterschiedlicher sensorischer Areale variiert zwischen unterschiedlichen Spezies. Die Teile, die besonders wichtig sind, nehmen einen großen Teil der Repräsentation ein, d.h. die Größe der cortikalen Repräsentation richtet sich nicht primär nach der Größe der Oberfläche dieses Körperteils, sondern nach der Anzahl der Sinnesrezeptoren in oder auf diesem Körperteil. Am Rücken finden sich nur wenige Rezeptoren, daher ist seine cortikale Repräsentation relativ klein, dagegen nehmen die Hände einen überproportional großen Teil der Körperrepräsentation ein. Die Größe der einzelnen Repräsentationen erlaubt daher gewisse Aussagen über Vorlieben der einzelnen Tierarten (Abb. 3). Offenbar sind das Reden und das Schmecken für Menschen relativ wichtig und auch das Berühren, während die Füße nicht mehr so wichtig sind wie bei unseren Vorfahren. Affenhände und -füße hingegen, wenn man diese Körperteile so bezeichnen darf, besitzen etwa gleich große Repräsentationen. Die Katze sieht ziemlich gefährlich aus, und das Kaninchen hat nichts als Essen im Sinn.

2 Kandel, E. R./Schwartz, H./Jessel, T. M.: Principles of Neural Science, New York 2000, S. 344, Abb. 18.6. 


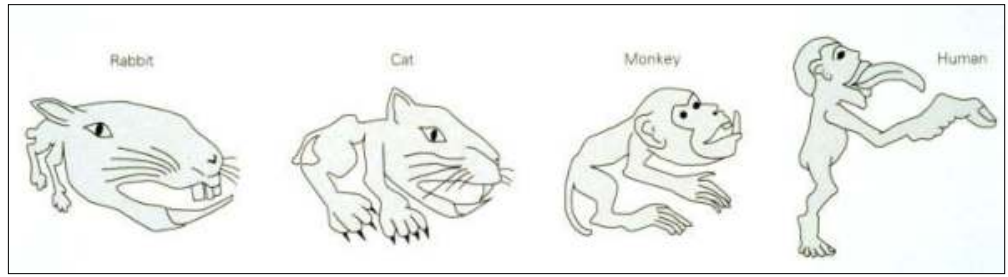

Abb. 3: Repräsentation des Körpers unterschiedlicher Tierarten im jeweiligen Cortex als Funktion der Repräsentationsgröße der entsprechenden Körperregion im sensomotorischen Cortex ${ }^{3}$

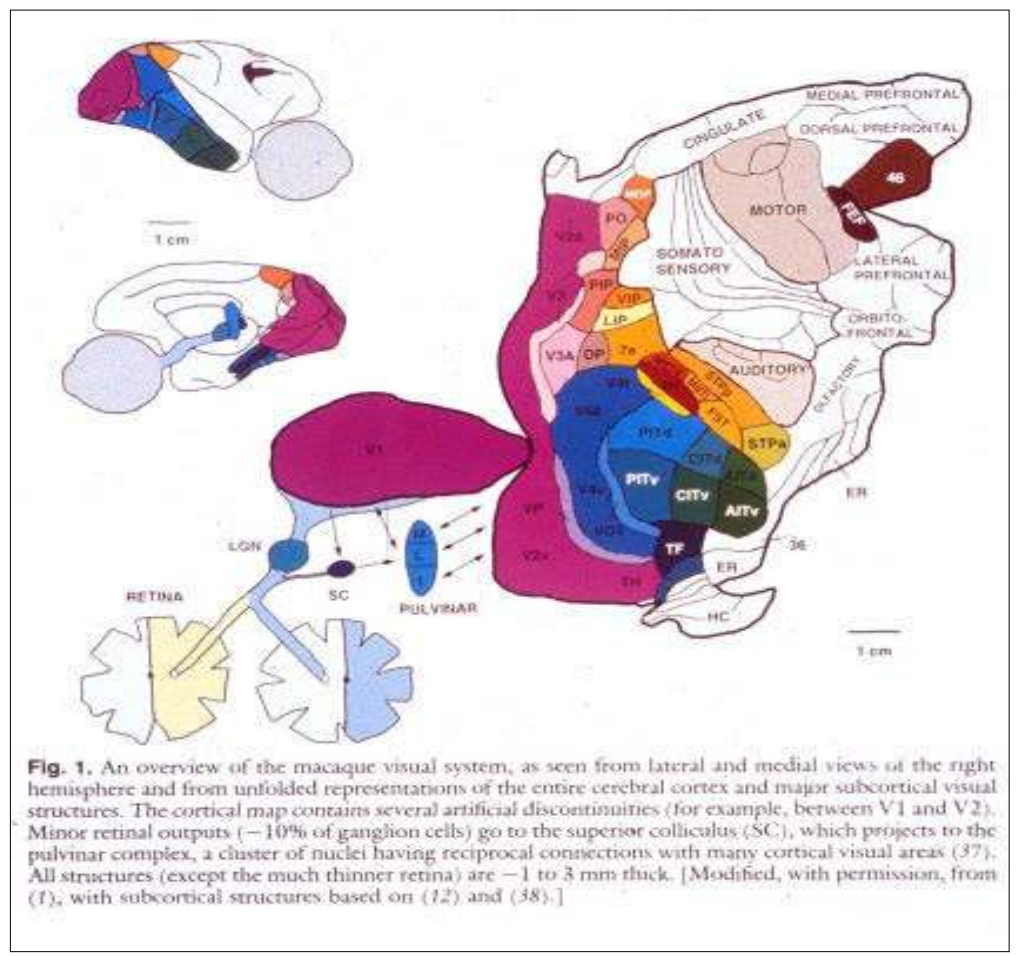

Abb. 4: Schematische Darstellung des Cortex eines Rhesusaffen einschließlich seiner Verbindung mit dem Auge ${ }^{4}$

3 Kandel/Schwartz/Jessel (wie Anm. 2), S. 388, Abb. 20.5.

4 van Essen, D. C./Anderson, C. H./Felleman, D. J.: „Information Processing in the Primate Visual System: An Integrated Systems Perspective“, in: Science, 255 (1992), S. 419-423, hier S. 420, Abb. 1. 
Die Spezialisierung des Cortex geht aber noch viel weiter. Wie oben bereits angedeutet, befasst sich der hintere, okzipitale Anteil des Cortex mit der Analyse visueller Informationen, also mit dem Sehen. Innerhalb dieser Spezialisierung auf das Sehen findet sich noch eine erstaunlich weitgehende Unterspezialisierung. Abb. 4 zeigt eine schematische Ansicht des Cortex eines Rhesusaffen und seine Verbindung mit dem Auge. Alle farbig unterlegten Anteile dieses Cortex haben direkt oder indirekt etwas mit der Analyse visueller Reize zu tun, wie man insbesondere aus Einzelzellableitungen weiß. Die Abbildung illustriert zwei wesentliche Erkenntnisse: Erstens, dass etwa ein Drittel des Cortex für Sehen, also die Analyse visueller Informationen verwendet wird. Der Mensch ist von der Evolution eher dafür vorgesehen, die visuelle Außenwelt zu analysieren, als für die Durchführung komplexer mathematischer Operationen. (Für Mathematik steht ein wesentlich geringerer Anteil des Gehirns zur Verfügung, deshalb sind Menschen in dieser Hinsicht auch Computern deutlich unterlegen.) Zweitens sehen wir aus der Abbildung, dass sich der Bereich des Gehirns, der sich mit visuellen Eingangserregungen befasst, in mindestens vierzig unterscheidbare Unterabteilungen aufgliedern lässt. Mit anderen Worten, der visuelle Cortex ist nicht monolithisch, sondern besteht aus einer großen Anzahl eindeutig voneinander abgrenzbarer Untereinheiten. Das bedeutet für den Sehvorgang, dass Sehen nicht eine Einheit darstellt, sondern modular aufgebaut ist, auch wenn wir subjektiv Sehen als Einheit empfinden. In Wirklichkeit besteht Sehen also aus vielen Teilfunktionen, die Teilaspekte der visuellen Umgebung analysieren. Im Augenblick ist noch nicht klar, aus welchen Teilaspekten Sehen im Einzelnen zusammengesetzt ist, und welche cortikalen Areale jedem dieser Teilaspekte zugeordnet sind. Sicher wissen wir, dass Farbensehen, Bewegungssehen und Tiefensehen, das heißt Abstandsschätzung, von zumindest teilweise unterschiedlichen Cortexarealen vermittelt werden. Ein bestimmter Teil des Cortex, der neben dem primären visuellen Cortex insbesondere Areale eher an der Basis des Gehirns umfasst, analysiert Farben, während Bewegungen insbesondere einen Anteil des Cortex am Übergang zwischen Scheitel- und Schläfenlappen aktivieren. 


\section{Einige allgemeine Prinzipien der Hirnfunktion: Ordnung}

Ein weiteres Prinzip der Informationsverarbeitung im Cortex ist das der Ordnung. Beim Blick in die visuelle Umwelt wird der rechte Anteil dieser Umwelt im linken Teil des Gehirns abgebildet und der linke Anteil in der rechten Hirnhälfte. Die Gründe dafür sind, zumindest mir, unklar. Eigentlich erscheint es unpraktisch, alle sensorischen und motorischen Signale in die jeweils gegenüberliegende Hirnhälfte zu leiten. Aber auch hierbei handelt es sich um ein allgemeines Prinzip im Tierreich, das auch auf den Menschen Anwendung findet und das möglicherweise auf einer Rotation des Kopfes um 180 Grad während der Entwicklungsgeschichte beruht. Der zentrale Bereich des Gesichtsfeldes, der in der Fovea der Netzhaut abgebildet wird, nimmt eine unverhältnismäßig große Projektion im primären visuellen Cortex ein (Abb. 5). Die Peripherie des Gesichtsfeldes hingegen, die offenbar weniger wichtig ist, beeinflusst nur einen wesentlich kleineren Anteil der cortikalen Oberfläche im primären visuellen Cortex. In Analogie zum oben erwähnten sensorischen und motorischen Homunculus werden also auch in diesem Sinne im cortikalen System die Anteile, die besondere Bedeutung haben, sehr ausführlich abgebildet. Noch klarer als bei dem somatosensorischen Homunculus aus Abbildung 3 ist das Prinzip der Ordnung. Benachbarte Orte der Außenwelt werden auf benachbarten Orten der Netzhaut abgebildet und dann auch an benachbarten Orten des primären visuellen Cortex repräsentiert. Diese Repräsentation ist natürlich stark verzerrt, aber sie bewahrt im Großen und Ganzen die Nachbarschaftsverhältnisse. Daher werden - mit Ausnahme der Bereiche in der Nähe des vertikalen Meridians, der die Repräsentationen der beiden Hirnhälften voneinander trennt - benachbarte Punkte der Außenwelt an benachbarten Bereichen des Cortex repräsentiert. Allerdings steht diese Repräsentation der visuellen Außenwelt gewissermaßen auf dem Kopf: Da bereits das Netzhautbild aus physikalischen Gründen auf dem Kopf steht und die Netzhautprojektion zum Cortex dies nicht kompensiert, steht auch die Repräsentation der Außenwelt im visuellen Cortex auf dem Kopf: Die oberen Anteile des Gesichtsfeldes werden im unteren Teil des primären visuellen Cortex repräsentiert, die unteren Gesichtsfeldanteile im oberen Cortexbereich. 


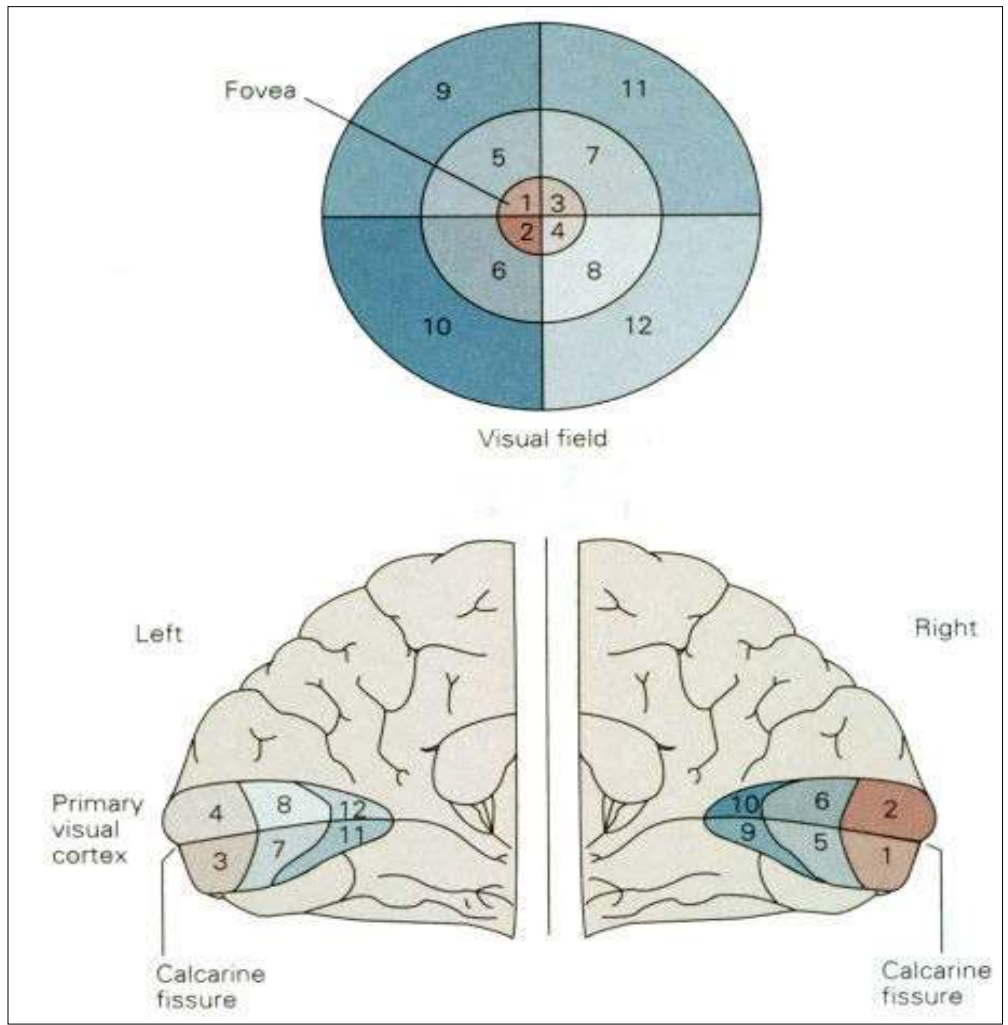

Abb. 5: Die Abbildung der visuellen Außenwelt im primären visuellen Cortex betont das Gesichtsfeldzentrum auf Kosten der Peripherie ${ }^{5}$

\section{Einige allgemeine Prinzipien: Parallelverarbeitung}

Ein drittes Prinzip der cortikalen Informationsverarbeitung ist das der Parallelverarbeitung. Dieses Prinzip ist eng verbunden mit dem der Spezialisierung. Denn da sich unterschiedliche Anteile des Cortex auf die Verarbeitung unterschiedlicher Aspekte der visuellen Welt spezialisiert haben, folgt daraus, dass unterschiedliche Merkmale eines Bildes gleichzeitig verarbeitet werden können. Auch das Prinzip der topographisch geordneten Projektion, das oben angesprochen wurde, bedeutet, dass für jeden Gesichtsfeldort ein eigener Cortexanteil zur Verfügung steht. Daher können viele Bereiche der Außenwelt gleichzeitig bezüglich jedes Merkmals verarbeitet werden. Durch diese massive simultane Parallel-

5 Kandel/Schwartz/Jessel (wie Anm. 2), S. 532, Abb. 27.9. 
verarbeitung verschiedener Orte des Gesichtsfeldes und innerhalb jedes Ortes nochmals für verschiedene Aspekte, wie Farbe, Bewegung und Tiefe wird eine erhebliche Beschleunigung der visuellen Verarbeitung erreicht. Das Gehirn ist im Vergleich zu Computern ein relativ langsames informationsverarbeitendes Gerät. Seine Taktfrequenz beträgt maximal 100 Hertz, eventuell sogar wesentlich weniger bis hinunter zu 10 Hertz, also 10 Prozesse pro Sekunde. Das ist im Vergleich zu einem modernen Computerprozessor mit über einem Gigahertz, also mehr als einer Milliarde Rechenschritte pro Sekunde, sehr langsam. Durch die Parallelverarbeitung wird diese Geschwindigkeitsbegrenzung zumindest teilweise wettgemacht. Dadurch sind Menschen bei der Verarbeitung natürlicher Szenen auch den besten Computern mindestens ebenbürtig, meist jedoch deutlich überlegen.

Andererseits verursacht die Parallelverarbeitung auch gewisse Probleme, denn die Parallelverarbeitung unterschiedlicher Merkmale der gleichen Szene, bzw. des gleichen Objekts in unterschiedlichen Arealen des Cortex bedeutet, dass diese Verarbeitung unterschiedlicher Merkmale zu einem gewissen Teil unabhängig voneinander stattfindet. Daher erhebt sich die Frage, wie die verteilte Verarbeitung der visuellen Information wieder zu einem einheitlichen Bild, d.h. einer einzigen Repräsentation aller Merkmale eines Objektes führen kann. Dieses Problem ist auch als das so genanntes Bindungsproblem bekannt und führte zu einer erheblichen Kontroverse innerhalb der an neurobiologischen Fragestellungen interessierten Forscher. Eine Reihe von Experimenten zeigt, dass es sich hierbei tatsächlich um ein Problem handelt. Unter bestimmten Bedingungen funktioniert die Zusammenführung der einzelnen Merkmale nämlich nicht perfekt. Zeigt man Versuchspersonen beispielsweise drei oder vier Buchstaben, etwa ein A, ein C, ein D und ein F in unterschiedlichen Farben, während ihre Aufmerksamkeit durch eine weitere Aufgabe abgelenkt wird, dann werden diese Versuchspersonen dennoch in der Lage sein, die dargebotenen Buchstaben nach Ende der Darbietung korrekt anzugeben. Sie werden auch in der Lage sein, die individuellen Farben der vier Buchstaben korrekt zu benennen. Es kommt aber relativ häufig zu Verwechslungen, d.h. zu falschen Zuordnungen zwischen Buchstaben und Farben. Während also die Angabe eines falschen Buchstabens, der nicht dargeboten wurde, oder einer falschen Farbe, also einer Farbe, die nicht dargeboten wurde, extrem selten ist, werden die Farben den Buchstaben häufiger falsch zugeordnet. Das führt dazu, dass beispielsweise ein rot dargebotenes $\mathrm{A}$ als grünes $\mathrm{A}$ und ein grün dargebotenes B als rotes B angegeben werden. Das Bindungsproblem sagt genau 
dieses Ergebnis voraus. Da Farbe und Form, also Orientierung, in gesonderten und voneinander teilweise getrennten Arealen des Cortex analysiert werden, ist es denkbar, dass sowohl Farbe als auch Form sämtlicher Buchstaben korrekt analysiert werden. Bei einer sehr kurzfristigen Darbietung und einer Ablenkung der Aufmerksamkeit gelingt es dem Cortex aber nicht immer perfekt, die Form und die Farbe einander zuzuordnen und es kommt zu den oben beschriebenen Verwechslungen.

Die Lösung des Bindungsproblems im Cortex ist zurzeit offen. Es existieren verschiedene Vorschläge, u.a. der einer zeitlichen Koppelung, die dazu führen sollte, dass jeweils die neuronalen Repräsentationen aller Attribute eines Objektes, wie seine Form, Farbe und sein Abstand gleichzeitig kurz aktiv sind, direkt gefolgt von einer ebenfalls kurzzeitigen Aktivierung aller Repräsentationen eines anderen Objektes. Eine alternative Vorstellung, die ich persönlich plausibler finde, besteht darin, das Verbinden der unterschiedlichen Merkmale über ihren Ort zu erzielen. Denn während in den „höheren“ cortikalen Arealen Zellen auf Merkmale innerhalb eines relativ großen Bereiches des Gesichtsfeldes antworten und insofern nicht wissen, wo genau sich ein rotes Merkmal im Gesichtsfeld befindet, ist der primäre visuelle Cortex, wie wir oben sahen, streng topographisch geordnet. Durch Rückgriff auf diese topographische Repräsentation im primären visuellen Cortex könnten also „höhere" Areale im Prinzip genaue Positionsinformationen erhalten. Mit anderen Worten: durch Rückkoppelung (Feedback) und laterale Verschaltung zwischen parallelverarbeitenden Cortexarealen könnte das Bindungsproblem gelöst werden.

\section{Allgemeine Prinzipien der Hirnfunktion: Laterale und Vertikale Interaktion}

Laterale Interaktion, d.h. Interaktion zwischen verschiedenen Nervenzellen auf der gleichen Stufe der Informationsverarbeitung sowie Rückkoppelung, d.h. Einwirkung von „höheren“ Verarbeitungsstufen auf vorangehende Stufen stellen zwei weitere, sehr wichtige Prinzipien der Informationsverarbeitung im Cortex dar. Während die Bedeutung der lateralen Interaktion schon vor etwa einem halben Jahrhundert erkannt wurde, wurde die Bedeutung der Rückkoppelung lange unterschätzt. Vor zwei Dekaden wurde die Signal- und Informationsverarbeitung auf den ersten cortikalen Stufen der visuellen Analyse noch weitgehend als rein hierarchisch angesehen. In einem rein hierarchisch organisierten System läuft die Information stets nur in einer Richtung, weitgehend ohne Rück- 
koppelung. Man stellte sich also vor, dass die Erregung der cortikalen Areale rein durch den Reiz determiniert und in einer Aufeinanderfolge relativ fixierter Operationen analysiert würde. Heute wissen wir, dass Rückkoppelung im Cortex eine sehr große Rolle spielt. In vielen Teilen des Gehirns ist die Anzahl der so genannten rekurrenten Fasern, die von „höheren“ zu vorangehenden Analyseebenen zurücklaufen, zumindest ebenso groß, wie die Anzahl der Fasern, die von den „niedrigen“ Analyseebenen nach oben projizieren. Dadurch kann das System sich flexibel an sehr unterschiedliche Aufgaben anpassen, und zwar schon auf den ersten Analyseebenen. Andererseits macht die Flexibilität und Plastizität der Verarbeitung bereits auf diesen „frühen“ cortikalen Analyseebenen die Untersuchung und Entschlüsselung der Funktion dieser Ebenen weitaus schwieriger, als früher angenommen. Denn wenn die Aktivität einer Ebene nicht rein durch den Reiz, sondern auch durch interne Aktivität des Gehirns bestimmt wird, dann wird die Voraussage der Aktivität eines Cortexareals für den Fall der Darbietung eines bestimmten Reizes sehr viel schwieriger, als wenn diese Cortexebene rein durch den äußeren Reiz aktiviert würde.

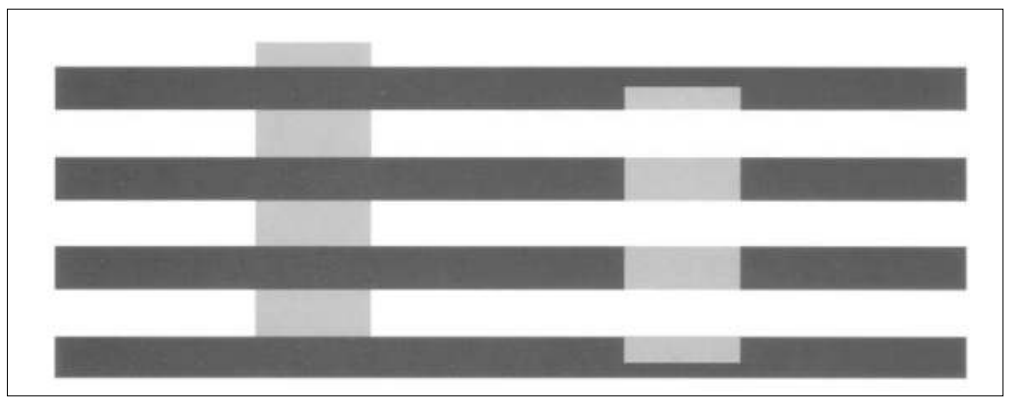

Abb. 6: Der Mechanismus der lateralen Interaktion führt beispielsweise zum Simultankontrast

Laterale Interaktionen, also die Wechselwirkungen zwischen benachbarten Zellen auf der gleichen Verarbeitungsebene, bestehen aus zwei Komponenten. Die erste, meist kurzreichweitige Interaktion ist die der gegenseitigen Unterstützung und Aktivierung. Die meiner Ansicht nach wichtigere und weitreichendere Interaktion ist die der lateralen Hemmung oder Inhibition. Dieser neuronale Mechanismus führt zu einer Reihe wohlbekannter Wahrnehmungstäuschungen, wie dem in Abb. 6 dargestellten Simultankontrast. Man kann sich leicht davon überzeugen, dass die zentralen Bereiche in der linken und rechten Hälfte der Abbildung die 
gleiche Intensität aufweisen. Aufgrund der unterschiedlichen Umgebungen führt in einem Fall die Übertreibung des Kontrastes durch laterale Inhibition zu einer Aufhellung des zentralen Bereiches, während sie im anderen Fall zu einer subjektiven Verminderung der wahrgenommenen Intensität führt.

\section{Wahrnehmung als Informationsreduktion und partielle Synthese}

Die Wahrnehmungstäuschung des Simultankontrastes ist nur ein Beispiel aus einer großen Anzahl von Täuschungen, die zeigen, dass unser Gehirn die Außenwelt nicht so abbildet, wie sie ist. Vielmehr interpretieren wir die Signale, die von den Sinnesorganen geliefert werden und synthetisieren im Kopf eine Abbildung der Außenwelt. Dabei verändern wir das Bild, das von den Objekten auf der Netzhaut abgebildet wird und modifizieren es so, dass es für das Gehirn besser handhabbar wird, beispielsweise durch Reduktion der Informationsmenge und durch Verschärfung und Verstärkung der Kontraste mittels lateraler Interaktion. Die Repräsentation der Außenwelt im Gehirn weicht also manchmal erheblich von der physikalisch erfassbaren Realität ab.

Meiner Ansicht nach sind die cortikalen Bilder, die wir uns von den Objekten der Außenwelt machen, insbesondere gekennzeichnet durch zwei Prinzipien: Nämlich dem der Reduktion von Informationen und dem der Konstruktion, also der Synthese von Repräsentationen. Reduktion ist deswegen erforderlich, weil die Sinnesorgane ständig so viele Signale anliefern, dass die Verarbeitungskapazität des Gehirnes durch die Rohdaten überfordert wäre. Das Gehirn muss also zunächst die Informationen komprimieren. Im visuellen Bereich kann man sich das vielleicht so vorstellen wie den Übergang von einer bit-map zu einem komprimierten Datenformat wie jpg. Es ist oft überraschend, wie stark Bildinformationen komprimiert werden können, ohne dass die subjektive Bildqualität leidet. Das Gehirn muss also Wichtiges von Unwichtigem trennen und sich bei der weiteren Analyse auf die Signale beschränken, die für die augenblickliche Situation wichtig sind.

Zusätzlich sind die Informationen, die von den Sinnesorganen geliefert werden, oft zweideutig. Dann muss auf der Grundlage allgemeiner Prinzipien und Vorannahmen über die Außenwelt eine Konstruktion und Synthese der augenblicklichen Situation erfolgen. Der Mensch interpretiert also das, was ihm die Sinnesorgane anliefern, um daraus eine möglichst konsistente Repräsentation der Außenwelt zu synthetisieren. Wir 
bilden die Außenwelt nicht nur ab, sondern konstruieren uns eine eigene Wirklichkeit, unsere eigene cortikale kleine Welt.

Ein wichtiger erster Prozess dieser Datenreduktion ist die primäre Begrenzung auf Formen und Konturen, unter Vernachlässigung von relativ gleichmäßigen Flächen. Es werden also aus dem von der Netzhaut angelieferten Bild zunächst die Konturen extrahiert, die meist den AuBengrenzen von Objekten entsprechen (leider manchmal auch weniger wichtigen Schatten). Dadurch wird das Bild der Außenwelt in erster Näherung auf eine Art von „Cartoon“ reduziert. Möglicherweise sprechen wir auch deshalb so gut auf Cartoons an, weil sie auf einer zweiten oder dritten Verarbeitungsstufe in unserem Gehirn der Repräsentation wirklicher Objekte schon recht nahe kommen.

Die einzelnen, lokalen Konturen dieses „Cartoons“ müssen nun miteinander in Beziehung gesetzt werden. Lokale Konturen müssen verknüpft werden, um voneinander abgrenzbare, geschlossene Konturlinien zu erzeugen, die ihrerseits Oberflächen und schlussendlich Objekte repräsentieren. Dieser Prozess der Kombination von Konturelementen zu Formen ist keinesfalls trivial, wovon wir uns leicht überzeugen können, wenn wir beispielsweise in einen Garten voller Äste und Blätter schauen. Eine Reihe recht einfacher Prinzipien unterstützen dieses Phänomen der Schaffung geschlossener Konturbereiche. Bereits auf der zweiten oder dritten Ebene der Informationsverarbeitung im visuellen Cortex werden aus unvollständigen Konturumrissen durch Interpolationen Formen ergänzt. Ein gutes Beispiel hierfür liefert Abb. 7A. Das für jedermann gut sichtbare Dreieck, dessen Intensität sich für Viele auch deutlich vom Hintergrund abhebt, ist physikalisch keinesfalls vollständig definiert. Die Linien, die das Dreieck definieren, brechen nach kurzem Verlauf ab. Man sieht hier also ein Dreieck, das gar nicht real existiert, das aber aufgrund von Vorwissen über die Außenwelt konstruiert wird. Aufgrund seiner Vorerfahrung ist für das Gehirn die einfachste und wahrscheinlichste Interpretation, dass die unterbrochenen Linien eigentlich weiterlaufen, aber durch ein davor liegendes Objekt verdeckt werden. 


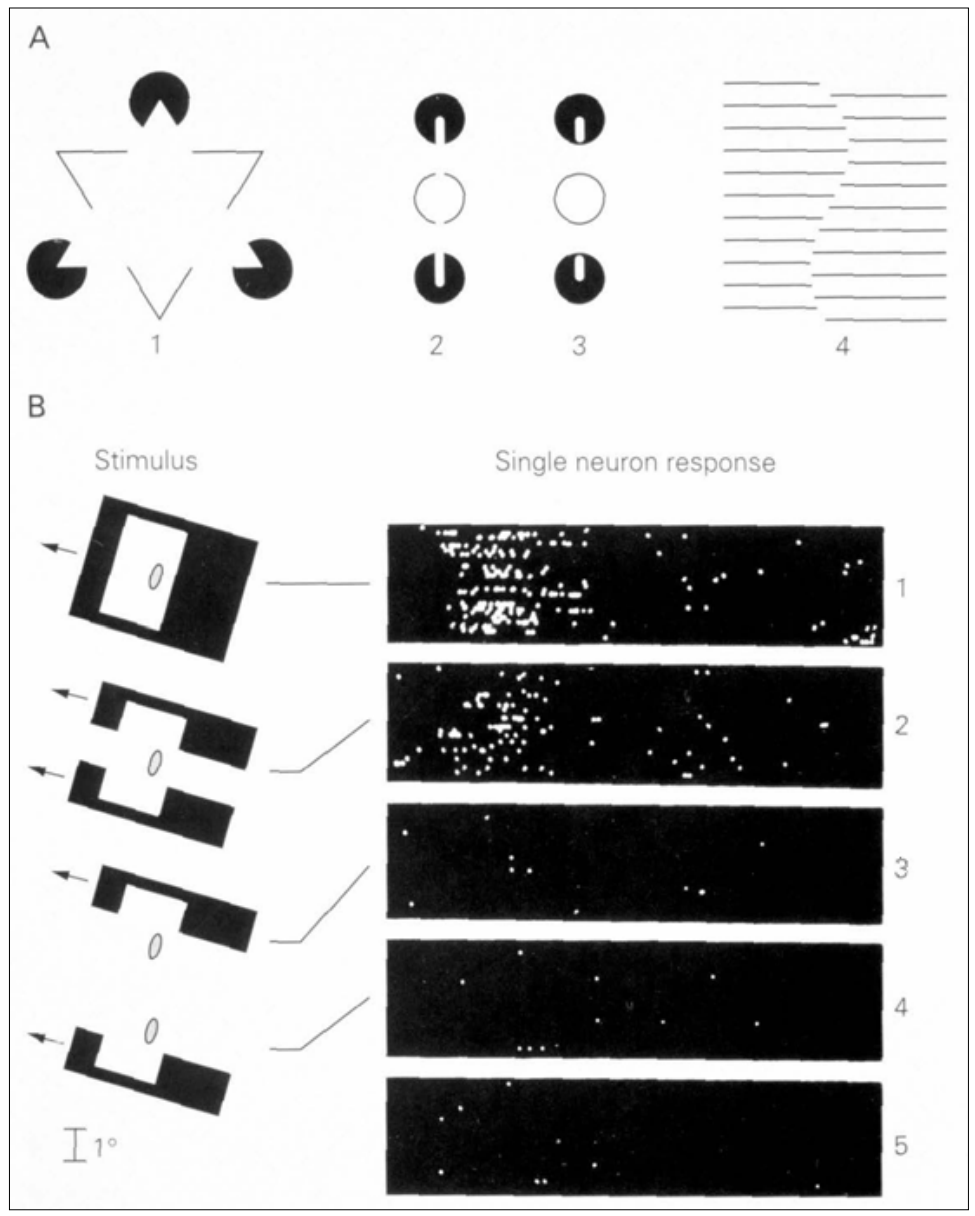

Abb. 7A und B: Synthese von ,kognitiven Konturen“ und durch solche Reize evozierte neuronale Aktivität ${ }^{6}$

Das aufregende an dieser Interpretation, die oft als „,kognitive Kontur“ bezeichnet wird, ist, dass sie anscheinend quasi „mechanisch“ auf einer frühen cortikalen Ebene, dem sekundären visuellen Cortex, erzielt wird. Auf dieser Ebene des visuellen Cortex findet man bei Einzelzellableitungen im Affengehirn Neurone mit rezeptiven Feldern, die, ähnlich wie im primären visuellen Cortex, das gesamte Gesichtsfeld in topographisch geordneter Weise abbilden. Das rezeptive Feld eines jeden Neurons ist definiert als jener Bereich des Gesichtsfeldes, entsprechend also jenem

6 Kandel/Schwartz/Jessel (wie Anm. 2), S. 564, Abb. 28.16. 
Bereich der Netzhaut, durch dessen Stimulation die Aktivität der Nervenzelle erhöht werden kann. Darbietung von Reizen außerhalb des rezeptiven Feldes soll die Aktivität des Neurons nicht erhöhen. Bewegt man eine Kante, also eine Kontur der für dieses Neuron optimalen Orientierung in der geeigneten Richtung durch das rezeptive Feld des Neurons, dann steigt die Entladungsfrequenz und somit die Aktivität dieses Neurons an (Abb. 7B). Jeder der Punkte in Abb. 7B1-7B5 symbolisiert einen so genannten „spike“, d.h. eine Entladung dieses Neurons. Die Zeitachse verläuft von links nach rechts, daher symbolisiert jede Zeile eine Bewegung des Reizes von rechts nach links. Die untereinander liegenden Zeilen geben die Ergebnisse von 20 Wiederholungen dieses Versuches wieder. Es ist offensichtlich, dass die Zelle ihre Aktivität erhöht, sobald die Kontur eine bestimmte Position erreicht hat. Wird der Reiz jetzt dergestalt modifiziert, dass ein Teil der Kontur entfernt wird, so dass das rezeptive Feld der Zelle eigentlich nicht mehr stimuliert wird, erhöht sich die Aktivität des Neurons trotzdem. Hier besteht also ein offensichtlicher Widerspruch: Eigentlich werden Neurone nur durch eine Reizdarbietung innerhalb ihres rezeptiven Feldes aktiviert, andererseits findet sich in Abb. 7B1 eine Aktivierung durch einen Reiz, der außerhalb des rezeptiven Feldes dargeboten wird. In Abb. 7B2-7B5 sind die Ergebnisse eines Kontrollexperimentes dargestellt. Wird nur der obere Teil oder nur der untere Teil des in Abb. 7B2 verwendeten Reizes dargeboten, dann folgt daraus keine nennenswerte Aktivierung des Neurons (Abb. 7B3-7B4). Insofern wurde das rezeptive Feld korrekt identifiziert, denn ein Reiz außerhalb des rezeptiven Feldes führt in der Tat nicht zu einer Aktivierung der Zelle (Abb. 7B5). Nur dann, wenn zwei Reize in einer Weise gezeigt werden, welche die „kognitive“ Interpretation nahe legt, dass sich auch im rezeptiven Feld des Neurons eine Kontur befindet, steigt die Aktivität des betreffenden Neurons.

Dieses Ergebnis zeigt, dass die Ergänzung fehlender „kognitiver“ Konturen bereits sehr früh im Verlaufe der visuellen Informationsverarbeitung stattfindet. Insofern finde ich persönlich den Begriff der ,illusionären Kontur“ besser als den der „kognitiven Kontur“. Durch diesen relativ einfachen Mechanismus der Kontur-Interpolation gelingt es dem Gehirn, teilweise verdeckte Konturen in einer quasi automatischen Weise zu ergänzen und auf diese Weise geschlossene Konturen als Grundlage der darauffolgenden Form- und Objekterkennung zu schaffen. Die Betonung liegt wiederum auf „schaffen“, denn auch hier werden die Signale aus der Außenwelt nicht einfach analysiert, sondern aufbereitet, um eine Repräsentation der Außenwelt zu synthetisieren. Dabei ergänzt das Ge- 
hirn Fehlendes (und häufig fehlt irgendetwas in visuellen Szenen, weil es zum Teil verdeckt ist durch davor liegende Objekte) nach bestem Wissen und Gewissen.

\section{Wahrnehmungstäuschungen als fehlgeschlagene Konstruktionen}

Die Konstruktion der Repräsentation der Außenwelt und insgesamt der subjektiven Wirklichkeit ruht demnach auf zwei Grundprinzipien. Einerseits wird diese Repräsentation der Außenwelt natürlich durch die Signale bedingt, die das Gehirn von den Sinnesorganen erhält. Zum anderen wird die Repräsentation aber auch Ausdruck der Erwartungshaltungen sein, mit denen das Gehirn diesen Sinnessignalen begegnet. Es wirken demnach so genannte „bottom-up“-Signale aus den Sinnesorganen und „top-down“-Informationen von höheren Ebenen der Signalverarbeitung zusammen. Zum Bereich der Erwartungshaltungen lässt sich neben den im Laufe des Lebens erworbenen Erfahrungen auch ein Teil des von der Gattung als Ganzem erworbenen Wissens über die Welt zählen und sicherlich die „Bedingungen der Möglichkeit von Wahrnehmung“, also die Kategorien wie Raum und Zeit, in denen wir all unsere Erfahrung organisieren. Diese Erfahrungswerte besagen beispielsweise, dass Licht normalerweise von oben kommt und Schatten nach unten wirft und hilft uns so, in Abb. 8 eine dreidimensionale Struktur zu erkennen, obwohl das gedruckte Bild selbstverständlich nur zweidimensional, also flach sein kann. Je nach Verlauf und Anordnung der Schatten scheint es sich bei den kreisförmigen Reizen der Abb. 8 entweder um kleine Hügel oder kleine Mulden zu handeln.

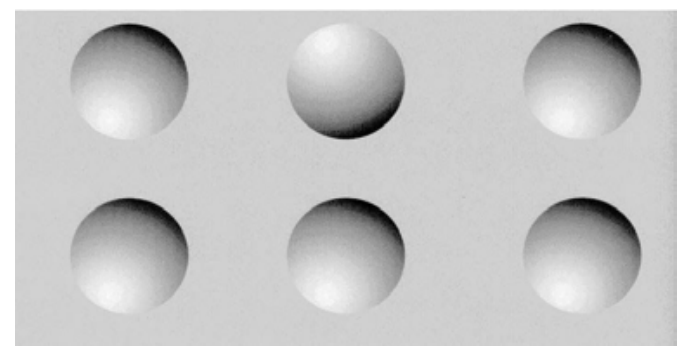

Abb. 8: Angedeutete Schatten erzeugen die Illusion von Tiefe: Das flache Blatt scheint ,"Mulden “ oder ,Hügel “ aufzuweisen. ${ }^{7}$

7 Seckel, A.: The Art of Optical Illusions, London 2000, S. 25, Abb. 16. 


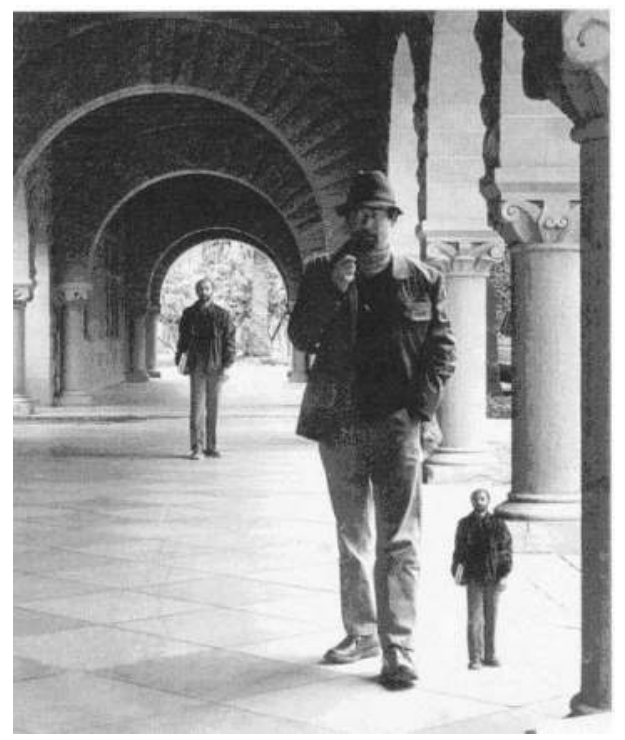

Abb. 9: Eine Größentäuschung, die auf Perspektive beruht ${ }^{8}$

„Top-down“-Signale erlauben uns auch eine perspektivische Interpretation der Netzhautbilder. Dieses Wissen hilft bei der Erzielung der Größenkonstanz. Objekte erzeugen mit zunehmendem Abstand vom Beobachter immer kleinere Bilder auf der Netzhaut. Aufgrund des Wissens über Perspektive erwarten wir diese Verminderung des Netzhautbildes und sind insofern in der Lage, sie wahrnehmungsmäßig zu kompensieren und zu verhindern, dass Objekte zu schrumpfen scheinen, wenn wir uns von ihnen entfernen. Das Wissen um Perspektive ermöglicht die Größenkonstanz, es erscheint nicht ungewöhnlich, dass der Herr links oben in Abb. 9 eine geringere Höhe aufweist, als der Herr im Vordergrund des Bildes. Kopiert man dagegen das Bild des Herrn links oben nach rechts unten in einen Bereich des Bildes, der aufgrund der perspektivischen Interpretation näher am Beobachter liegt, dann erscheint die gleiche Abbildung des Herrn als wesentlich zu klein. Diese ,top-down“- Beeinflussung der wahrgenommenen Größe vollzieht sich automatisch und ist nicht dem Willen unterworfen. Wir können uns also nicht gegen diese perspektivische Deutung wehren. Das zweidimensionale Bild wird dreidimensional interpretiert und führt zu einem intrinsischen Widerspruch und zu einer Wahrnehmungstäuschung.

8 Seckel (wie Anm. 7), S. 45, Abb. 36. 
Ganz allgemein beruhen viele Wahrnehmungstäuschungen genau darauf, dass die üblichen Interpretationsmechanismen in spezifischen Situationen versagen und es zu offensichtlichen Diskrepanzen zwischen der subjektiven Wahrnehmung und der physikalischen Außenwelt kommt. Wahrnehmungstäuschungen sind also vielfach Fehlschläge der cortikalen Konstruktion oder Synthese der Repräsentation von „Welt“. Daneben gibt es natürlich eine Reihe anderer Ursachen für Wahrnehmungstäuschungen, aber viele lassen sich in der Tat als Diskrepanzen zwischen der subjektiv konstruierten Repräsentation einer bestimmten Szene einerseits und dem physikalischen Wissen über die Struktur der Welt andererseits interpretieren. Häufig lässt sich jeder Bereich des Bildes getrennt problemlos interpretieren, die einzelnen Bereiche können jedoch nicht in ein gemeinsames Ganzes integriert werden.

Die Abb. 10a illustriert diese Hypothese. Auf den ersten Blick scheint es sich um eine Treppe zu handeln. Je nach Vertrautheit mit dieser Art von Täuschungen wird dem Beobachter aber sehr schnell klar, dass die dreidimensionale Interpretation dieses zweidimensionalen Bildes fehlschlägt. Es ist nicht möglich, sich ein dreidimensionales Objekt vorzustellen, das ein solches zweidimensionales Bild erzeugen würde. In Wirklichkeit existieren durchaus Objekte, die derartige zweidimensionale Bilder erzeugen. Das Gebilde erscheint nur deshalb unmöglich, weil bestimmte Voraussetzungen und Vorannahmen gewissermaßen automatisch in unsere Interpretation eingebaut sind. Der Betrachter nimmt unmittelbar an, der Grundriss des Gebildes sei rechtwinklig, entspräche also einen Quadrat oder zumindest einem Rechteck. Bei genauerer Betrachtung der Abbildung wird man bemerken, dass zwar alle Stufen etwa die gleiche Größe haben, dass aber die Anzahl der Stufen pro Seite unterschiedlich hoch ist. Diese Beobachtung ist ein deutlicher Hinweis darauf, dass es sich in Wirklichkeit nicht um ein Gebilde mit einem rechtwinkligen Grundriss handelt. Sobald man die Annahme der Rechtwinkligkeit aufgibt, ist es durchaus möglich, ein Objekt zu bauen, dass aus einem bestimmten Blickwinkel die in Abbildung 10a dargestellte zweidimensionale Projektion erzeugt. 


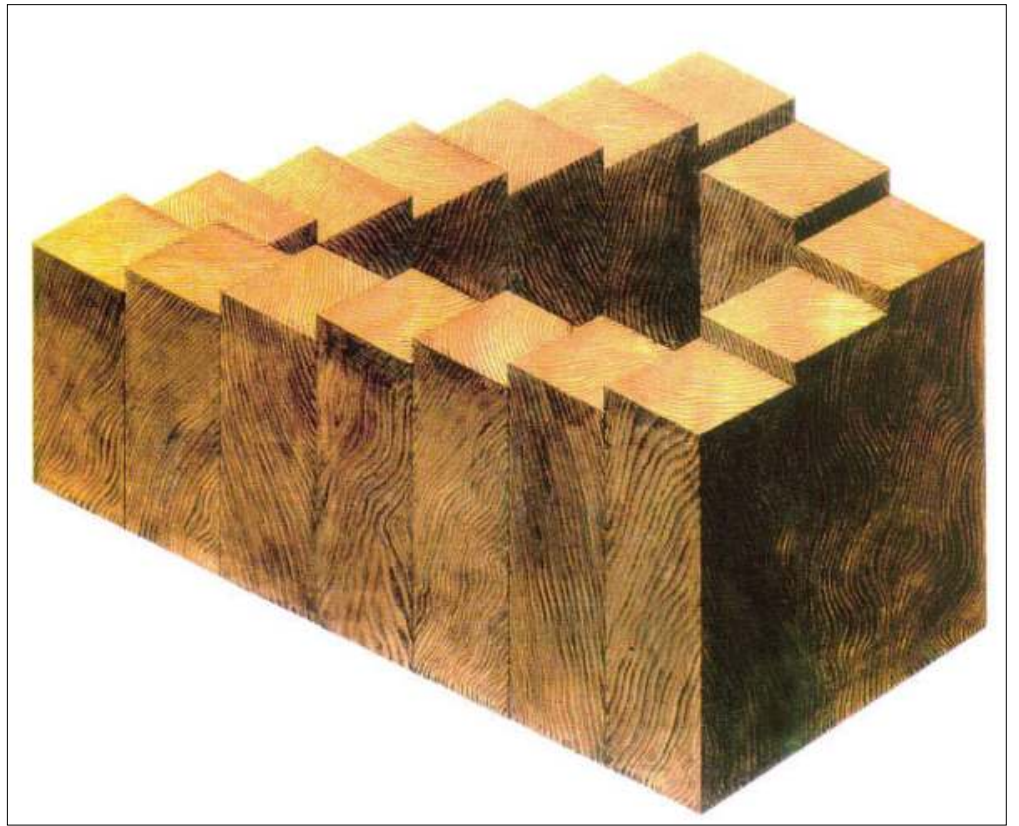

Abb. 10a: Eine „unmögliche“ Treppe nach L. S. und R. Penrose $1958^{9}$

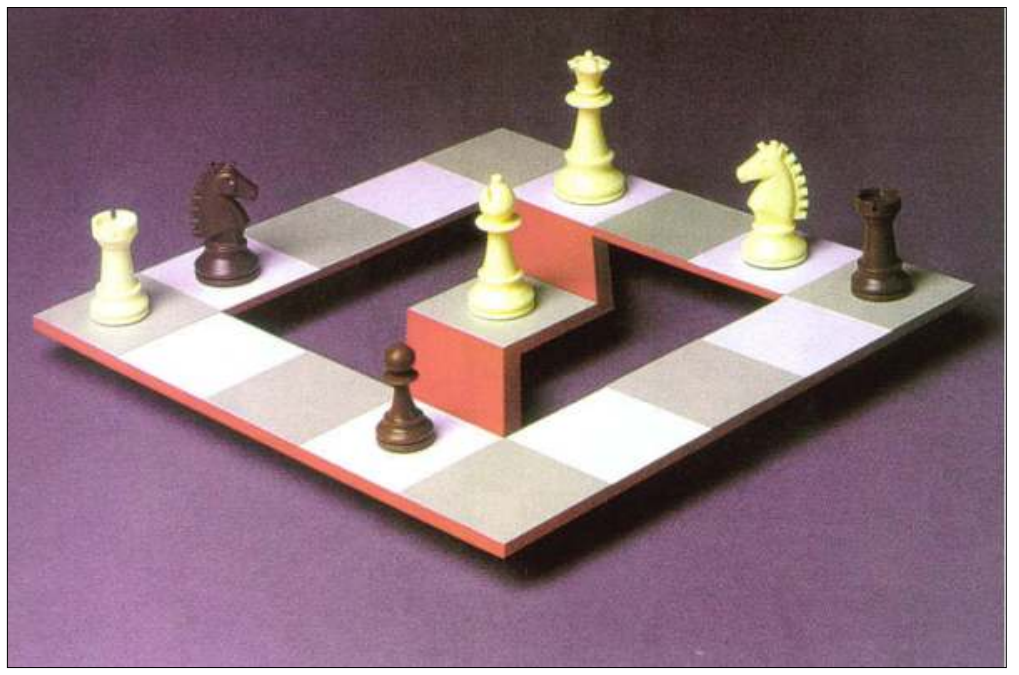

Abb. 10b: Ein ,unmögliches “ Basis-Schachbrett ${ }^{10}$

9 Seckel (wie Anm. 7), S. 21, Abb. 12. 


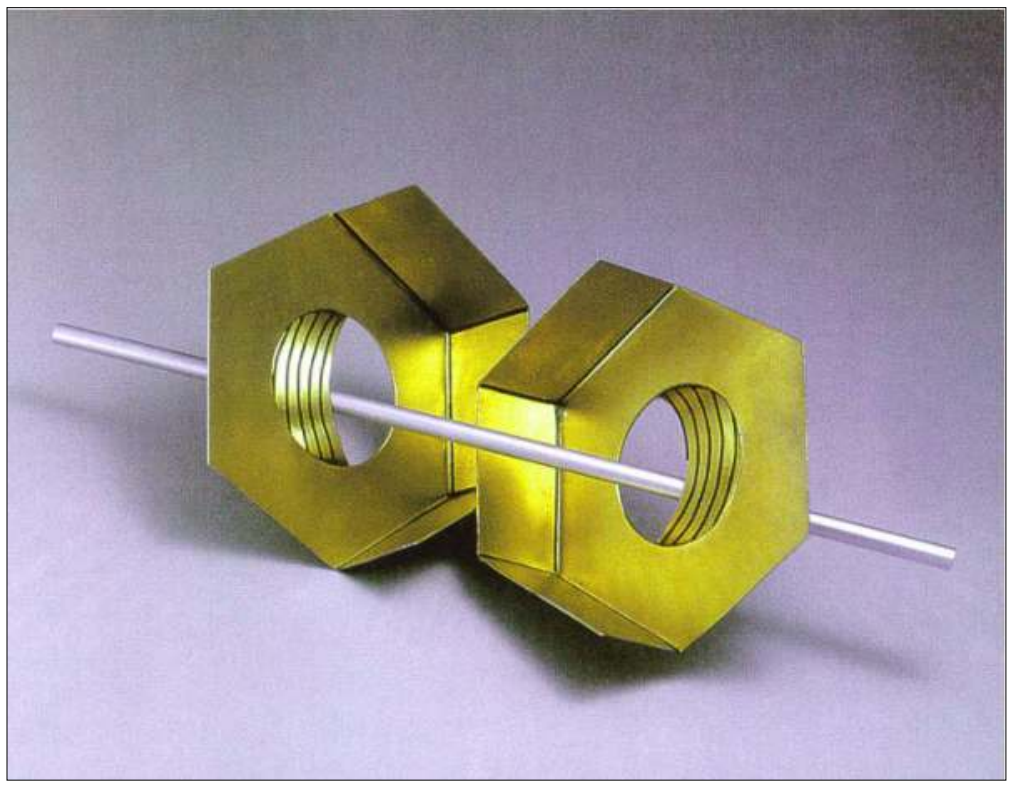

Abb. 10c: Eine ,unmögliche“ Trilogie ${ }^{11}$

Die Überraschung ist vermutlich größer bei Betrachtung von Abb. 10b, da diese Wahrnehmungstäuschung weniger bekannt ist. Im ersten $\mathrm{Au}-$ genblick erschreckt auch diese Abbildung nicht. Dann aber fällt eine Diskrepanz auf zwischen der flachen Anordnung der Elemente im äußeren Quadrat einerseits, und der Stufe zwischen dem oberen und unteren Teil der Abbildung andererseits. Der Betrachter hat das Gefühl, dass ein solches Objekt nicht möglich ist. Wieder wird die Illusion dadurch ausgelöst, dass normalerweise perfekt funktionierende Mechanismen der Interpretation und Synthese von Repräsentationen dreidimensionaler Objekte aus zweidimensionalen Bildern fehlschlagen. Insbesondere angesichts der rechtwinkligen Welt, in der wir den größten Teil unseres Lebens verbringen, erwarten wir rechte Winkel in vielen Abbildungen der Außenwelt. Daher interpretieren wir die Zickzacklinien, welche die obere und die untere Reihe der Quadrate verbinden, als Begrenzungen einer Stufe, die in der Realität mehreren rechten Winkeln entsprechen. Das Gehirn erwartet aufgrund seiner Vorerfahrungen, dass in der Realität eine Stufe existiert, die aus dem gegebenen Betrachtungswinkel die

10 Seckel (wie Anm. 7), S. 50, Abb. 39.

11 Seckel (wie Anm. 7), S. 18, Abb. 9. 
zweidimensionale Repräsentation einer Zickzacklinie erzeugt. Sobald sich der Beobachter von dieser fehlerhaften Interpretation freimacht und die gezackte Linie nicht mehr als Hinweis auf eine Stufe interpretiert, ist das vorliegende Objekt nicht mehr „unmöglich“. Doch die unwillkürlichen Annahmen in unserem visuellen System sind so stark, dass es den meisten Beobachtern sehr schwer fällt, sich von der Interpretation „Stufe“ freizumachen. (Es hilft etwas, sich klar zu machen, dass eine wirkliche Stufe weiter links enden müsste.) Nur durch einen Bruch, vergleichbar vielleicht einem Medienumbruch, machen wir uns von unserer erlernten und eingeübten Interpretation frei und erst dann können wir diese Abbildung realitätsangemessener wahrnehmen.

Noch offensichtlicher ist das Fehlschlagen der dreidimensionalen Synthese von Objekten aufgrund von unzutreffenden Vorannahmen in Abb. 10c. Der visuelle Cortex geht aufgrund langjähriger Erfahrungen davon aus, dass lange dünne Objekte, die auf einer zweidimensionalen Abbildung geradlinig verlaufen, auch in der dritten Ebene nicht gebogen, sondern gerade sind. Diese Interpretation der metallisch aussehenden Stange in Abbildung 10c stimmt aber nicht mit der perspektivischen Interpretation der relativen Lage der beiden Schrauben überein. Wieder schlägt die Synthese eines dreidimensionalen Abbildes auf der Basis der zweidimensionalen Darstellung fehl. Der Beobachter muss sich von der Annahme freimachen, dass die in der Papierebene gerade eingezeichnete Linie auch in der dritten Dimension, senkrecht zur Papierebene, gerade verläuft. Sobald man annimmt, dass sich die Stange in verschiedenen Abschnitten des Bildes in unterschiedlichen Distanzen zum Beobachter befindet, löst sich der scheinbare Widerspruch auf, der im Bild inhärent zu sein scheint. Selbstverständlich kann man ein solches Objekt in der Realität bauen. Die Täuschung wird nur aus einem bestimmten, kleinen Blickwinkel möglich sein; sobald sich der Beobachter nach oben oder unten bewegt, wird die Biegung der Stange in der dritten Dimension erkennbar werden.

Die letzte Demonstration dieser fehlgeschlagenen dreidimensionalen Rekonstruktion findet sich in Abb. 11. Das dreidimensionale Gebilde, das der Betrachter hier in der Hand hält, kann nur dann physikalisch existieren, wenn die senkrechten Verbindungsstäbe nicht etwa, wie man zunächst annehmen möchte, parallel zur Papierebene verlaufen. Sie müssen eindeutig in der dritten Dimension gebogen sein. Sobald sich der Betrachter dies klar macht, wird das zuvor unmögliche Objekt möglich. 


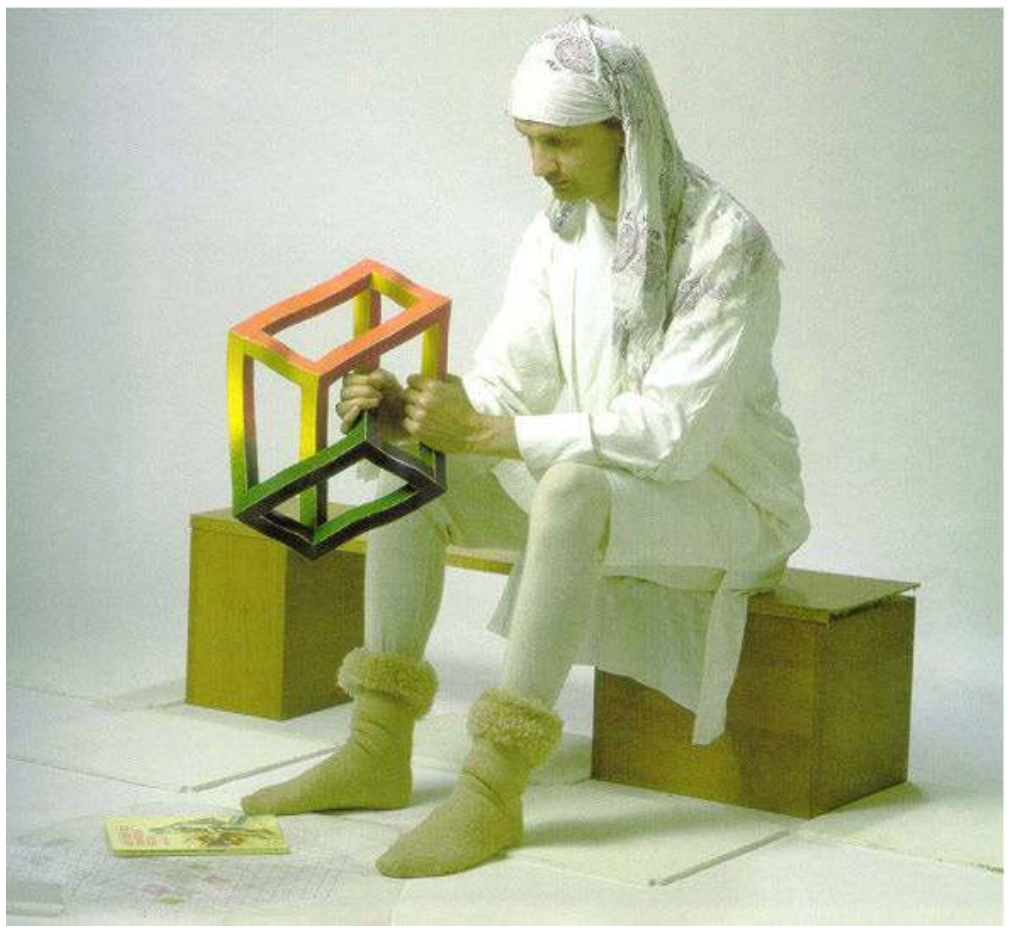

Abb. 11: Ein ,unmöglicher" Würfel samt verzweifeltem Betrachter ${ }^{12}$

Zusammenfassend können wir festhalten, dass das menschliche Gehirn auf der Basis der Signale, die es von den Sinnesorganen über die Außenwelt erhält, eine relativ gut funktionierende Repräsentation der Außenwelt synthetisiert. Sowohl die persönliche Geschichte als auch die Geschichte der jeweiligen Gattung sorgt dafür, dass grobe Fehlwahrnehmungen der Außenwelt ausgemerzt werden; sei es dadurch, dass das Individuum lernt oder dadurch, dass es an der Fortpflanzung gehindert wird. Trotz dieser erstaunlichen Übereinstimmung zwischen Außenwelt und cortikaler Repräsentation ist die Repräsentation der Außenwelt nicht perfekt. Angesichts der Unterbestimmtheit der Signale aus den Sinnesorganen und angesichts der Notwendigkeit, die Menge der Signale zu komprimieren, ist es nicht überraschend, dass die Synthese der Repräsentation nicht immer perfekt ist und hin und wieder von der physikalischen Realität abweicht. Wahrnehmungstäuschungen sind ein gutes Anschauungsmaterial dafür, dass die Wahrnehmung der Außenwelt keine

12 Seckel (wie Anm. 7), S. 14, Abb. 5. 
reine Analyse darstellt, sondern teilweise Synthese auf der Basis nicht vollständig ausreichender und gleichzeitig nicht komplett analysierbarer Sinnessignale ist. Täuschungen, die allen Menschen gemeinsam sind, bezeichnen wir als Sinnestäuschungen; solche, die privater Natur sind, d.h. von anderen Mitgliedern der Spezies nicht geteilt werden, nennen wir Halluzinationen.

\section{Stufen der visuellen Wahrnehmung und ihre Störungen}

Wir sahen eingangs, dass das Gehirn kein Computer ist, sondern auf einer Reihe von Funktionsprinzipien aufbaut, wie Parallelverarbeitung, laterale Interaktion und vertikale Integration (Rückkoppelung), die sich in Computern in der Regel nicht finden. Das Gehirn synthetisiert, auf der Grundlage von Sinnesinformationen und von Vorwissen, eine Repräsentation der Außenwelt, die recht gut mit dieser übereinstimmt, aber keinesfalls perfekt ist, wie uns die Wahrnehmungstäuschungen zeigen. Es bestehen Hinweise darauf, dass die Art der Synthese zwischen unterschiedlichen Individuen variiert, und es finden sich signifikante Unterschiede bereits auf der Ebene der Photorezeptorenverteilung. Konturen sind, wie oben ausgeführt, besonders interessant für das visuelle System. Diese Konturen können beispielsweise durch Unterschiede in der Beleuchtungsintensität, der Farbe, des Abstandes, der Bewegung oder der Textur (von Oberflächen) definiert werden. Diese unterschiedlich definierten Konturen werden von teilweise unterschiedlichen Anteilen des Cortex detektiert, und zwar auf einer lokalen Basis. Neurone im (primären) visuellen Cortex sprechen also auf Kontraste in ihrem jeweiligen rezeptiven Feld an und detektieren auf diese Art und Weise lokale Kantenelemente. Diese lokalen Kanten müssen zu Formen kombiniert werden, die einzelne Objekte definieren. Die Gestaltpsychologen beschäftigten sich stark mit den Mechanismen oder Gesetzen, die der Kombination einzelner Konturelemente zu geschlossenen Formen und Gestalten zugrunde liegen und konnten einige allgemeine Mechanismen und Regeln für diesen Prozess der Kombination von Elementarreizen zu Gestalten angeben, beispielsweise das „Gesetz der guten Form“ oder das des ,gemeinsamen Schicksals“ bewegter Elementar-Konturen. Eine zweite Stufe nach der der reinen Konturdetektion besteht also in der Kombination lokaler Konturelemente zu Formen und Oberflächen.

Ein weiteres, teilweise getrenntes System scheint dafür zu sorgen, dass die Innenbereiche dieser Formen in der jeweils korrekten Farbe 
wahrgenommen werden. Diese ersten Stufen der visuellen Informationsverarbeitung können mehr oder weniger selektiv gestört werden, z. B. durch cortikale Tumoren oder Infarkte sowie durch Vergiftungen, z.B. Kohlenmonoxydvergiftungen. Nach Zerstörung bestimmter cortikaler Bereiche kann ein Patient beispielsweise keine Farben mehr unterscheiden. Die Welt erscheint aufgrund des Defektes nur noch in Grautönen, ähnlich einem Schwarzweißfilm. Während also Läsionen der Netzhaut sowie des Sehnerven und der Sehbahn dazu führen, dass der Patient in einem bestimmten Bereich des Gesichtsfeldes überhaupt nichts mehr wahrnimmt, dort also blind ist, führen diese cortikalen Läsionen teilweise zu selektiveren Funktionsverlusten. Nicht nur die Fähigkeit, Farben zu unterscheiden kann selektiv ausfallen, sondern auch die Fähigkeit, Entfernungen richtig einzuschätzen oder auch die Bewegungen wahrzunehmen. Ausfälle auf dieser frühen Ebene der Informationsverarbeitung, die zum Ausfall einer Teilleistung führen, möchte ich als Indiskriminationen (indiscriminations) bezeichnen. ${ }^{13}$ Diese Störungen führen auch dazu, dass eine rein durch Bewegung, Tiefe oder eventuell Farbe definierte Kontur vom Patienten nicht mehr detektiert werden kann.

Auf der nächsten Verarbeitungsebene werden die einzelnen lokalen Konturen zusammengefasst zu Formen, wie oben beschrieben. Auch diese Verarbeitungsstufe kann mehr oder weniger selektiv gestört werden. Man spricht vom Krankheitsbild der apperzeptiven Agnosie. Patienten, die unter einer apperzeptiven Agnosie leiden, verhalten sich unter Umständen so, als seien sie blind, obwohl sie noch sehen. Ihre Netzhaut ist intakt und sie sind in der Lage, lokale Konturen zu erkennen. Aber da sie nicht mehr in der Lage sind, diese Konturen miteinander zu gruppieren, können sie keine Formen und insofern auch keine Objekte mehr wahrnehmen. Das führt dazu, dass die Patienten nicht mehr in der Lage sind, selbst einfachste Bilder abzuzeichnen. Beispielsweise wird die Kopie eines „Strichmännchens“ nicht viel Ähnlichkeit mit dem Vorbild haben. Daher sind die Patienten schwerst gestört, und es wäre interessant zu untersuchen, ob sie dennoch in der Lage sind, ästhetische Urteile abzugeben. Denn es ist bekannt, dass aufgrund der Parallelverarbeitung im visuellen System Patienten sich teilweise in ihrer Umgebung zurechtfinden können, obwohl sie keine Formen mehr erkennen. Insofern wären ästhetische Urteile solcher Patienten zwar überraschend aber letztlich nicht völlig auszuschließen.

13 Fahle, M.: „Failures of visual analysis: scotoma, agnosia, and neglect“, in: Fahle, M./Greenlee, M. (Hrsg.): The neuropsychology of vision, Oxford 2003, S. 179-258. 
Ist die Stufe der Kombination lokaler Konturen zu Formen intakt, besteht die Aufgabe darin, die so gebildeten Formen und Oberflächen mit den Repräsentationen zuvor erkannter und abgespeicherter Objekte in Verbindung zu bringen. Nur dadurch ist es möglich, „Sinn“ aus dem jeweils auf der Netzhaut abgebildeten Bild zu machen. Denn es reicht nicht aus, eine Oberfläche an einem bestimmten Bereich des Gesichtsfeldes zu erkennen, sondern für die Generierung von Handlungen müssen Menschen wissen, um welche Art von Objekten es sich in ihrer Umgebung handelt. Diese Stufe der Verbindung aktuell vorliegender Repräsentationen von Objekten der Außenwelt mit im Gedächtnis gespeicherten Repräsentationen von Objekten kann ebenfalls relativ selektiv gestört sein. Diese Störung wird als assoziative Agnosie bezeichnet. Die betroffenen Patienten sind im Gegensatz zu den apperzeptiven Agnostikern durchaus in der Lage, mäßig komplexe Strichzeichnungen zu kopieren. Sie erkennen also zumindest einfache Formen und können sie kopieren. Sie sind aber nicht in der Lage, die Zeichnungen zu erkennen in dem Sinne, dass sie die Bezeichnung oder die Verwendung des soeben abgezeichneten Gegenstandes angeben könnten. Diese Patienten erkennen also Oberflächen, es fehlt aber die Verbindung zu Gedächtnisinhalten und somit zum Wissen über Objekte. Dies kann zum einen darauf beruhen, dass diese Gedächtnisinhalte verloren gegangen sind (dann kann der Gegenstand auch beim Betasten nicht erkannt werden). Wesentlich häufiger aber fehlt ,lediglich“ die Verbindung zu den abgespeicherten Gedächtnisinhalten. Dann ist der Patient durchaus dazu fähig, ein Objekt durch Betasten zu erkennen - nicht aber bei visueller Darbietung, obwohl der Gegenstand abgezeichnet werden kann. Man könnte sich durchaus vorstellen, dass Patienten mit dieser Art von Störungen in der Lage sind, ästhetische Urteile abzugeben.

Eine weitere Untergruppe von Patienten kann nicht nur Punkte bei der Gesichtsfeldprüfung erkennen, sondern auch Konturen detektieren, sie zu Formen zusammensetzen und diesen Formen Objekte aus dem Gedächtnis zuordnen, erkennt sie also. Diese Patienten sind auch in der Lage, den Gebrauch des Objektes pantomimisch zu beschreiben oder auf das Bild eines Objektes der gleichen Kategorie zu zeigen, sie können das Objekt aber nicht benennen. Es ist ein auch im Alltagsleben so genannter Normalpersonen nicht unerhörtes Phänomen, dass die Bezeichnung eines Objektes sozusagen ,,auf der Zunge liegt“", das Wort aber kurzfristig nicht gefunden wird. Die beiden letztgenannten Gruppen von Patienten zeigen, dass offensichtlich eine gewisse Trennung zwischen der Repräsentation von Informationen über Objekte und den Bezeichnungen dieser Objekte 
besteht. Es kann dabei isoliert lediglich der Zugang von der Objekterkennung zum zugehörigen Wort gestört sein (so dass der Patient das Objekt beispielsweise nach Betasten richtig benennen kann, das Wort selbst also noch vorhanden ist), was man als „Anomie“ bezeichnet. Es kann aber auch bereits die Information über die Eigenschaften des Objektes verloren gegangen sein, bzw. der Zugriff auf diese Informationen vom visuellen Eingang her, wie oben geschildert. In diesem Falle spricht man von einer Agnosie. Sowohl agnostische als auch und insbesondere anomische Patienten sollten in der Lage sein, weitgehend normale ästhetische Urteile abzugeben.

Mit der Identifikation eines Objektes ist in der Regel der eigentliche Sehvorgang abgeschlossen. Die weitere Verarbeitung bezieht sich dann auf die Benennung und auf emotionale und ästhetische Bereiche die Identifikation eines Objektes ist zumindest partiell unabhängig von seiner Benennung und Bewertung. Hiermit ist der Überblick über die Verarbeitungsebenen des visuellen Systems abgeschlossen. Das in diesem Abschnitt Gesagte soll helfen, die Funktion und insbesondere die Funktionsstörungen bzw. Begrenzungen der visuellen Wahrnehmung besser zu verstehen.

\section{Gehirnaktivität und Wahrnehmung}

In den vorhergehenden Abschnitten wurden zwei Beispiele dafür angeführt, dass die übliche, präzise Repräsentation der Außenwelt im Gehirn fehlschlagen kann. Wenn Teile des visuellen Systems ausfallen, dann kann, wie oben beschrieben, die Analyse der von der Netzhaut stammenden Signale auf unterschiedlichen Stufen enden und eine adäquate Repräsentation der visuellen Außenwelt bleibt aus. Auch Wahrnehmungstäuschungen stellen Fehlschläge der adäquaten Repräsentation der Außenwelt dar, beispielsweise beruhend auf einer inadäquaten Synthese des Wahrnehmungseindruckes auf der Basis der Sinnesinformationen. Die im Rahmen von Wahrnehmungstäuschungen auftretenden Fehlrepräsentationen sind jedoch allen, oder fast allen, Menschen gemeinsam. Dies gilt nicht für alle Patienten.

Beispielsweise werden Patienten mit einer Farbsinnschwäche wie

der „Rot-Grün-Blindheit“ bestimmte Szene und Bilder deutlich anders wahrnehmen als ihre farbtüchtigen Mitmenschen. Noch stärker ist die Abweichung der Wahrnehmung zwischen Normalpersonen und solchen Patienten, die unter Halluzinationen leiden. Halluzinationen sind sozusagen private Sinnestäuschungen, die von den Mitmenschen nicht geteilt 
werden. Diese Halluzinationen können sehr lebhaft sein und vom Patienten nur schwer oder gar nicht von der Wirklichkeit unterschieden werden. Schizophrene Patienten beispielsweise leiden unter Halluzinationen, die sie nicht als solche identifizieren können. Diese Patienten akzeptieren die Halluzinationen, zumindest teilweise, als Teil der Wirklichkeit. Ihr Gehirn produziert also Repräsentationen von Objekten der Außenwelt, die nicht existieren oder zumindest nicht in dieser Form zum angegebenen Zeitpunkt vorhanden sind. Die Patienten haben auch nicht die Möglichkeit, zwischen einer Halluzination und der Wirklichkeit zu unterscheiden; d.h., zwischen einer Synthese der Repräsentation, die auf validen Sinnessignalen beruht und einer Synthese, die einer Grundlage in den Signalen der Sinnesorgane entbehrt.

Andere Patienten, beispielsweise nach Defekten auf der Ebene der Netzhaut, insbesondere in deren Zentrum (Makulopathie), leiden unter Halluzinationen, die z.T. sehr stark ausgeprägt sein können, die sie aber jedenfalls als Halluzinationen einordnen können. Diese Patienten berichten dann beispielsweise, sie sähen Zwerge oder Menschen herumlaufen, von denen sie wissen, dass sie nicht real vorhanden sind. Diese Symptome sind weniger selten, als man vermuten könnte, da diese Patienten häufig nicht über ihre Symptome sprechen, aus Angst, für verrückt gehalten zu werden. Ich persönlich bin der Ansicht, dass diese Symptome dadurch bedingt sind, dass der visuelle Cortex nach Ausfall des zentralen Sehens nicht mehr ausreichend mit Signalen von den Sinnesorganen versorgt wird. Ähnlich wie bei der sensorischen Deprivation, bei der Probanden ohne visuelle und akustische Stimulation in einem körperwarmen Wassertank liegen und nach einiger Zeit zu halluzinieren beginnen, bewirkt auch die sensorische Deprivation durch den Ausfall der Sinnesrezeptoren solche erstaunlich strukturierten Halluzinationen vermutlich dadurch, dass sich der mit Eingangssignalen unterversorgte Cortex seine Wahrnehmungen vollständig selbst generiert.

Auch aufgrund anderer Experimente ist bekannt, dass die elektrische Aktivität des Cortex, und das gilt auch für den primären visuellen Cortex, zu jedem Zeitpunkt nur zu einem relativ geringen Teil durch den jeweils dargebotenen Reiz bedingt wird. Ein wesentlicher Anteil der Aktivitätsmuster beruht dagegen auf so genannter intrinsischer Aktivität, d.h., auf den Eingangssignalen anderer Cortexareale und auf der unmittelbar vorangehenden Aktivität im (visuellen) Cortex selbst. Diese Aktivität wird durch die Signale aus den Sinnesorganen lediglich moduliert, um die jeweiligen Repräsentationen der Außenwelt zu erzeugen. Die Hirnaktivität ist also zu jedem Zeitpunkt selbst in den so genannten sen- 
sorischen Arealen durch die dargebotenen Reize und gleichzeitig in erheblichem Ausmaß gewissermaßen durch die Erwartungshaltung des Cortex bedingt, also durch den Zustand des Cortex zum Zeitpunkt der Reizdarbietung. All diese Phänomene und Ergebnisse unterstreichen die zentrale Aussage, dass Wahrnehmung zu einem großen Teil Synthese ist und insofern Schönheit im Auge, oder besser im Hirne des Betrachters begründet liegt, denn ästhetische Urteile werden vermutlich in der Regel erst nach Abschluss des Prozesses der Objekterkennung abgegeben werden.

\section{Wahrnehmung als elektrische Aktivitätsmuster des Gehirns}

Die moderne Hirnforschung geht davon aus, dass Wahrnehmungen durch die elektrische Aktivität in Neuronenverbänden entstehen. Diese Annahme beruht darauf, dass bestimmte Nervenzellen im Gehirn ihre Aktivität erhöhen, sobald die für sie adäquaten Reize präsentiert werden. Doch ist diese Annahme wirklich gerechtfertigt? Beruhen die Wahrnehmungen tatsächlich auf der (elektrischen) Aktivität von Neuronenverbänden? Man könnte argumentieren, dass die elektrische Aktivität lediglich ein Epi-Phänomen der Wahrnehmung darstellt, nicht aber ihre Grundlage. Der begrenzte Nachweis der Kausalität kann in diesem Falle durch eine Reihe von Experimenten erbracht werden.

Beispielsweise ist es möglich, den Cortex des Menschen mit Hilfe sehr kurz dauernder lokaler Magnetfelder zu erregen. Erregung des motorischen Cortex führt dann typischerweise zur Bewegung von Muskelgruppen, beispielsweise des Armes, Beines oder Gesichtes. Erregung des visuellen Cortex führt dagegen typischerweise zur Wahrnehmung von umschriebenen Lichtblitzen oder Phosphenen. Wilder Penfield ${ }^{14}$ hat während Hirnoperationen bei Patienten umschriebene Teile des Cortex elektrisch stimuliert und konnte auf diese Art und Weise Wahrnehmungen und Erinnerungen induzieren. Im letzten Jahrzehnt hat man darüber hinaus Experimente, insbesondere bei Rhesusaffen, durchgeführt, bei denen der Einfluss von elektrischer Stimulation des Cortex auf die Wahrnehmung sehr viel besser quantifiziert werden konnte, als das beim Menschen möglich ist. Beispielsweise wurde ein Affe darauf trainiert, zwischen der Bewegung einer Punktwolke nach rechts und einer Bewegung

14 Penfield, W.: „Some observations on the cerebra cortex of man“, in: Proceedings of the Royal Society of London, 134 (1947), S. 329-350. 
nach links zu unterscheiden. Dies ist eine leichte Aufgabe, wenn alle Punkte in die gleiche Richtung wandern. Bewegt sich jedoch nur ein kleiner Teil der Punkte in die Vorzugsrichtung, wird der Affe häufig nicht in der Lage sein, die richtige Antwort zu geben und er ist gezwungen zu raten. Das führt dazu, dass für Korrelation „Null“ der Affe ebenso oft auf die linke wie auf die rechte Taste drückt. Auch bei geringen Bewegungen nach rechts wird in der Regel signifikant häufiger die rechte Taste gedrückt, jedoch häufig auch fälschlicherweise die linke Taste. Mit zunehmender Korrelation wird die Anzahl der Fehler abnehmen. Bill Newsome und Mitarbeiter suchten sich nun im Cortex dieses Affen einen Bereich, in dem die Neurone stark auf eine Bewegung des Reizes nach rechts antworteten. Dann injizierten sie einen schwachen Strom in diesen Bereich des Affencortex, während die gleichen Reize wie zuvor dargeboten wurden. Durch die Strominjektion veränderte sich die durchschnittliche Reaktion des Affen signifikant. Bei unkorrelierten Reizen wurde jetzt überdurchschnittlich häufig die rechte Taste gedrückt, bei leicht nach links bewegten Reizen hielten sich die Knopfdrücke rechts und links in etwa die Waage (vgl. Abb. 12). Dieses Ergebnis zeigt, dass durch eine relativ grobe Maßnahme wie die Injektion eines Stromes in einen Bereich mit mehreren hundert Nervenzellen die Wahrnehmung des Affen gezielt verändert werden kann. Das Tier nimmt eine Punktwolke, die unbewegt dargeboten wurde, als nach rechts bewegt wahr. Daher kann man mit hoher Sicherheit schlussfolgern, dass in der Tat die elektrische Aktivität des Cortex die Grundlage der Wahrnehmung darstellt, und nicht etwa ein Epi-Phänomen, denn wie sollte die Induktion eines EpiPhänomens die genuine Wahrnehmung verändern können? Die elektrische Stimulation kleiner Cortexbereiche kann also, zumindest bei Affen, gezielt die Wahrnehmung visueller Reize verändern und auch die Reaktion auf diese Reize. Die Untersuchungen von Penfield und auch die mit transkranieller Magnetstimulation deuten darauf hin, dass die Verhältnisse beim Menschen nicht wesentlich anders sind. 


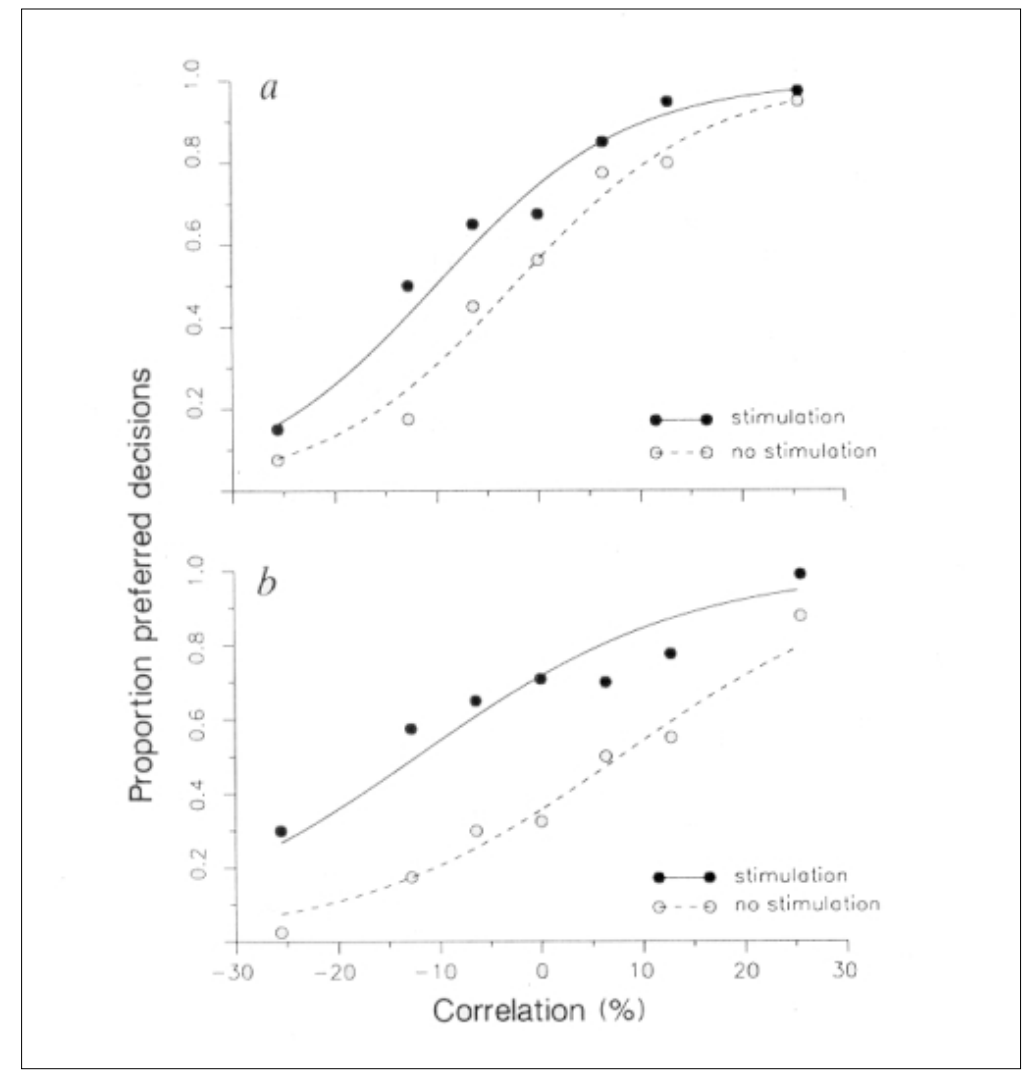

Abb. 12: Strominjektion in den Affencortex bewirkt eine Veränderung der wahrgenommenen Bewegungsrichtung ${ }^{15}$

\section{Wahrnehmung und Aufmerksamkeit}

Die Repräsentation der Außenwelt beruht also auf elektrischer Aktivität von Neuronenverbänden. Verschiedene Teile des Cortex sind spezialisiert auf die Lösung unterschiedlicher Aufgaben und sind jeweils (topographisch) geordnet, d.h. benachbarte Abschnitte der Außenwelt bzw. ähnliche Reize sind in benachbarten Teilen des Cortex repräsentiert. Der Cortex verarbeitet die Sinnesreize weitgehend parallel: Die Reize werden

15 Salzmann, D. C./Murasugi, C. M./Britten, K. H./Newsome, W. T.: „Microstimulation in visual area MT: effects on direction discrimination performance“, in: Journal of Neuroscience, 12, 6 (1992), S. 2331-2355, hier S. 2336, Abb. 4. 
auf verschiedene Cortexareale verteilt, die über laterale und vertikale Integration miteinander wechselwirken. So existieren innerhalb des Cortex Spezialisierungen nicht nur für Sehen und Hören, sondern innerhalb des Sehcortex nochmals vielfältige Unterspezialisierungen, beispielsweise für Farbensehen, Bewegungssehen oder die Wahrnehmung von Tiefe. Zusammenfassend lässt sich festhalten, dass Wahrnehmung keine bloße Analyse darstellt, sondern stets auch Synthese ist und dass die wahrgenommenen Bilder auf neuronalen Aktivitätsmustern beruhen, so dass den Objekten der Außenwelt in unserem Gehirn unterschiedliche Muster neuronaler Aktivität gegenüberstehen, die im Prinzip nichts, aber auch gar nichts, mit den Objekten der Außenwelt als solche zu tun haben. Diese beiden Bereiche - Welt und Repräsentation - sind sozusagen ontologisch getrennte Entitäten.

Es erhebt sich aber die Frage nach der Entsprechung von Repräsentationen und Objekten. Denn die Repräsentationen der Außenwelt scheinen ja relativ gut zu funktionieren, sonst würden unsere Interaktionen mit der Außenwelt häufiger fehlschlagen. Offenbar hat die Evolution dafür gesorgt, dass nicht adäquate Repräsentationen der Außenwelt ein rasches Ende finden. Aber das bedeutet keinesfalls, dass die Repräsentationen stets tatsächlich ein getreues Abbild der Außenwelt darstellen und dass wir uns auf die Abbildung der Außenwelt in unserem Kopfe verlassen können. Zusätzlich zu den bereits vorgestellten Wahrnehmungstäuschungen, die relativ unabhängig von der Zuwendung der Aufmerksamkeit sind, existieren eine Reihe von Phänomenen, bei denen wesentliche Inhalte der Außenwelt von der bewussten Wahrnehmung ausgeschlossen oder zumindest nicht bewusst repräsentiert werden, dass die Aufmerksamkeit ihnen nicht zugewandt wird. Ein bekanntes Beispiel besteht darin, einer Gruppe von Beobachtern die Aufgabe zu stellen, die Anzahl der Ballabgaben in einer Gruppe von Spielern zu beobachten und zu zählen. Die Ballabgaben folgen relativ rasch hintereinander. Versuchspersonen, die sich gut auf diese Aufgabe konzentrieren, bemerken in der Regel nicht, wenn sich ein als Gorilla verkleideter Mensch unter die Spieler mischt und sich kurz den Beobachtern zuwendet, auf die Brust schlägt und dann wieder aus dem Gesichtsfeld verschwindet. Wird die gleiche Szene noch einmal dargeboten, ohne dass die Beobachter eine spezifische Aufgabe haben, dann nehmen die Beobachter den Menschenaffen unmittelbar wahr und können nicht glauben, ihn bei der ersten Darbietung übersehen zu haben.

Nachbilder sind ein weiterer Hinweis darauf, dass die Repräsentation der Außenwelt nicht notwendigerweise übereinstimmt mit dieser 
Außenwelt. Schaut man eine Weile lang auf einen stationären visuellen Reiz, und danach auf eine homogene Fläche, dann bemerkt man ein Nachbild, in der Regel in der Gegenfarbe des zuvor betrachteten Reizes. Betrachtet man beispielsweise das Negativ eines Portraits, bei dem man in der Regel die Person nicht identifizieren kann, dann kehren sich die Kontraste im Nachbild um, und man erkennt die Person problemlos. Früher ging man davon aus, Nachbilder seien primär eine Folge der Ermüdung von Neuronenpopulationen. In den letzten Jahren stellte sich aber zunehmend heraus, dass viele der Nachbilder nicht rein durch eine Ermüdung der spezifisch gereizten Neuronenpopulationen erklärbar sind. Einige Nachbilder lassen sich nämlich deutlich länger konservieren, wenn nach der Adaptation nicht sofort ein Test folgt, sondern die Augen geschlossen bleiben, was nicht mit der Erklärung der Ermüdung und nachfolgenden Erholung von Neuronenpopulationen erklärbar ist. Rekalibrierung als Ergebnis sensorischer Erfahrung scheint demnach eine Rolle bei der Entstehung der Nachbilder zu spielen. Diese Rekalibrierung läuft relativ schnell $\mathrm{ab}$ und benötigt einige Zeit, um rückgängig gemacht $\mathrm{zu}$ werden. Nach längerer Betrachtung eines Wasserfalles wird ein unbewegtes Objekt nicht mehr als stationär, sondern als nach oben bewegt wahrgenommen. Diese Illusion beruht vermutlich darauf, dass der „Nullpunkt" des Systems für Bewegungswahrnehmung geändert wurde: Die stationäre Bewegungsrichtung, also null Bewegungsgeschwindigkeit, wird als Bewegung in die Gegenrichtung interpretiert, weil der Nullpunkt für Bewegungswahrnehmung in Richtung des Wasserfalls verschoben wurde.

Eine ähnliche Illusion lässt sich durch Abb. 13 generieren. Schaut man zunächst für längere Zeit, etwa 30 Sekunden, auf das linke Rechteck dieser Abbildung und danach auf das mittlere, dann wird die wahrgenommene Orientierung der oberhalb und unterhalb des Rechtecks angeordneten Streifen sich nicht mehr entsprechen, sondern die Streifen werden in verschiedene Richtungen geneigt erscheinen. Ebenso kann die Anzahl der Linien dieser beiden Gittermuster sich scheinbar ändern, wenn man für längere Zeit das rechte Rechteck fixierte. Nach Wechsel des Blickes auf das mittlere Rechteck scheint die Anzahl der Streifen in dem Bereich, der zuvor wenige Streifen sah, anzusteigen, während er in dem Bereich, der zuvor viele Streifen sah, abzunehmen scheint. 


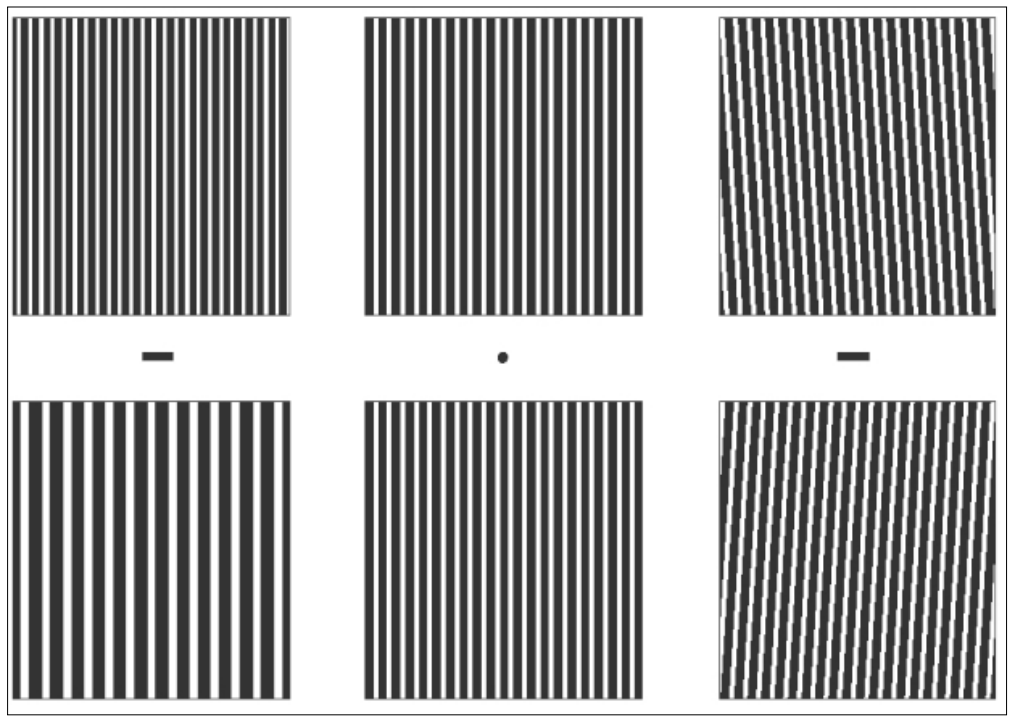

Abb. 13: Änderung der wahrgenommenen Orientierung als Nacheffekt: Nach Adaptation durch Blick auf das linke Rechteck für etwa 30 Sekunden und anschließende Fixation des mittleren Punktes scheint sich die Neigung der über und unter dem mittleren Punkt dargestellten Linienmuster zu unterscheiden. Entsprechendes gilt nach Adaptation auf dem rechten Rechteck für die wahrgenommene Gitterbreite des Musters ober- und unterhalb des mittleren Punktes. ${ }^{16}$

\section{Die Mehrdeutigkeit der Sinnesinformationen}

Mehrfach wurde angesprochen, dass Wahrnehmung nicht bloße Analyse, sondern auch Synthese von Repräsentationen der Außenwelt darstellt. Warum ist überhaupt eine solche Synthese erforderlich - könnte nicht eine funktionierende, adäquate und korrekte Repräsentation der Außenwelt aus den Signalen der Sinnesorgane extrahiert werden? Die beiden zweidimensionalen (flachen) Bilder unserer beiden Netzhäute beinhalten nicht alle erforderlichen Informationen, um eine dreidimensionale Welt sicher zu rekonstruieren. Es muss also nicht vorhandene Information synthetisiert werden. Das geschieht auf der Basis von Vorwissen. Solche Verwendung von Vorinformationen ist auch in Abb. 14 offensichtlich. Der Mensch hat sich daran gewöhnt zu wissen, dass eine Linie stets die

16 Karnath, H. O./Thier, P.: Neuropsychologie, Berlin 2003, S. 64, Abb. 4.28ac. 
Begrenzung eines Objektes darstellt. Es widerspricht diesem Vorwissen anzunehmen, dass die gleiche Linie zwei Objekte begrenzen könnte. Daher nehmen wir entweder die Vase oder aber das Gesicht wahr, jedoch nicht beides gleichzeitig. Eine Synthese aus These und Antithese ist in diesem Fall nicht möglich; diese Aufgabe überfordert das menschliche Gehirn.

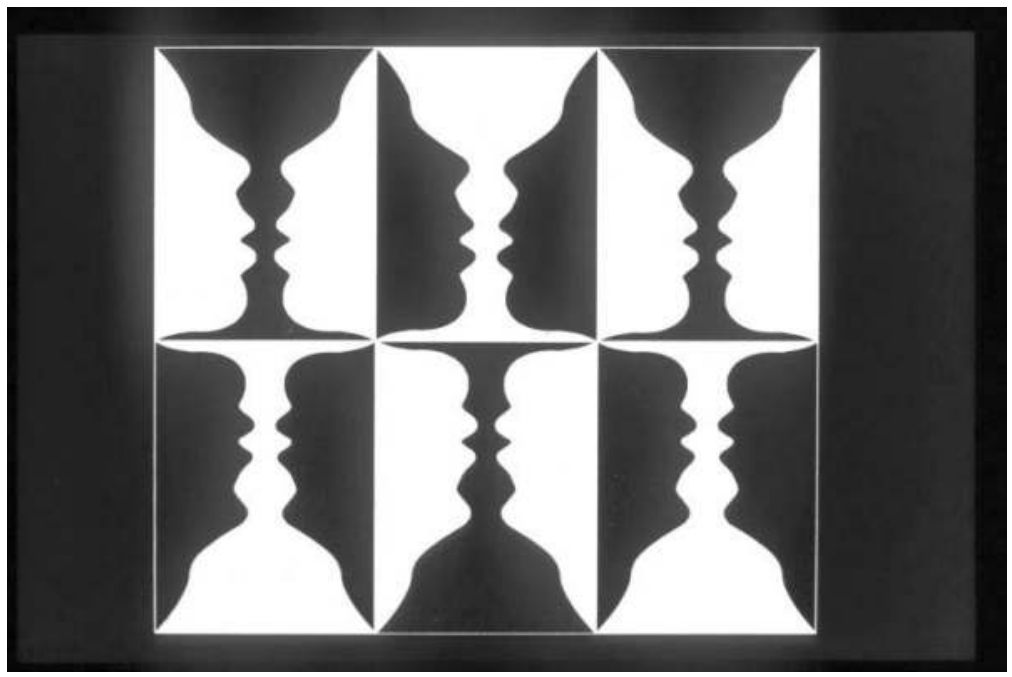

Abb. 14: Vase oder Gesichter - es können in der Regel nicht beide Interpretationen zugleich wahrgenommen werden. ${ }^{17}$

\section{Unbewusste Wahrnehmung}

Im Laufe der Jahrzehnte hat man sich an den Gedanken gewöhnt, dass Vieles im Gehirn passiert, von dem der jeweilige Besitzer nichts weiß. Atmung, Regelung des Blutdrucks und dergleichen mehr werden vom Hirnstamm verlässlich gesteuert, ohne dass das bewusste Ich sich hiermit auseinandersetzen muss. Seit Freud wissen wir, dass auch unsere Motivationen uns, zumindest teilweise, verborgen bleiben. Mittlerweile stellt sich zusätzlich heraus, dass Menschen nicht einmal immer wissen, welche Reize von ihrem visuellen Cortex wahrgenommen werden. Selbst im visuellen Cortex vollziehen sich Operationen bei der Wahrnehmung visueller Reize, von denen der Besitzer des Cortex nichts erfährt.

17 Rubin, E.: Visuell wahrgenommene Figuren, Kopenhagen, Berlin, London 1921. 
Ein beunruhigendes Beispiel ist in Abb. 15 dargestellt. Zunächst wird ein Pfeil für kurze Zeit dargeboten, der entweder nach links oder nach rechts zeigt. Nach einer variablen Pause wird ein zweiter, größerer Pfeil dargeboten, der den ersten Pfeil umschließt. Dieser zweite Pfeil zeigt entweder in die gleiche Richtung wie der erste, oder aber in die Gegenrichtung. Wird der zweite Pfeil unmittelbar nach dem ersten dargeboten, dann beträgt die Reaktionszeit etwa $350 \mathrm{~ms}$. Die Versuchsperson drückt also auf den rechten Knopf, wenn der zweite Pfeil nach rechts zeigt und auf den linken Knopf, wenn dieser Pfeil nach links zeigt. Vergeht eine zunehmend längere Pause zwischen den Darbietungen des ersten und des zweiten Pfeiles, dann nehmen die Reaktionszeiten der Versuchspersonen kontinuierlich zu, falls die Richtungen der beiden Pfeile sich nicht entsprechen. Wenn also der erste Pfeil nach rechts, der zweite Pfeil nach links zeigt, dann nimmt die Reaktionszeit mit zunehmendem Intervall zwischen den beiden Reizdarbietungen zu. Dies ist insbesondere deshalb überraschend, als die Versuchspersonen nicht in der Lage sind, zwischen den Richtungen zu unterscheiden, in die der erste Pfeil zeigte. Das bewusste Ich der Versuchspersonen ist also nicht in der Lage, die Richtung des ersten Pfeiles anzugeben, aber dennoch kann dieser Pfeil die Reaktionszeit der Versuchspersonen massiv verlängern. Im Gegenzug wird die Reaktionszeit bei Übereinstimmung der Richtungen beider Pfeile mit zunehmendem Intervall zwischen den beiden Reizen beschleunigt. Offensichtlich bereitet der Cortex nach der Darbietung des ersten Pfeiles eine Reaktion vor, die im Falle einer übereinstimmenden Richtung die Reaktion beschleunigt, im anderen Falle aber aufgrund einer Art von Umprogrammierung die Reaktion verlangsamt. Dieses Phänomen an sich wäre nicht überraschend, überraschend ist die Tatsache, dass die Information über die Richtung des ersten Pfeiles dem bewussten Ich nicht zur Verfügung steht. 


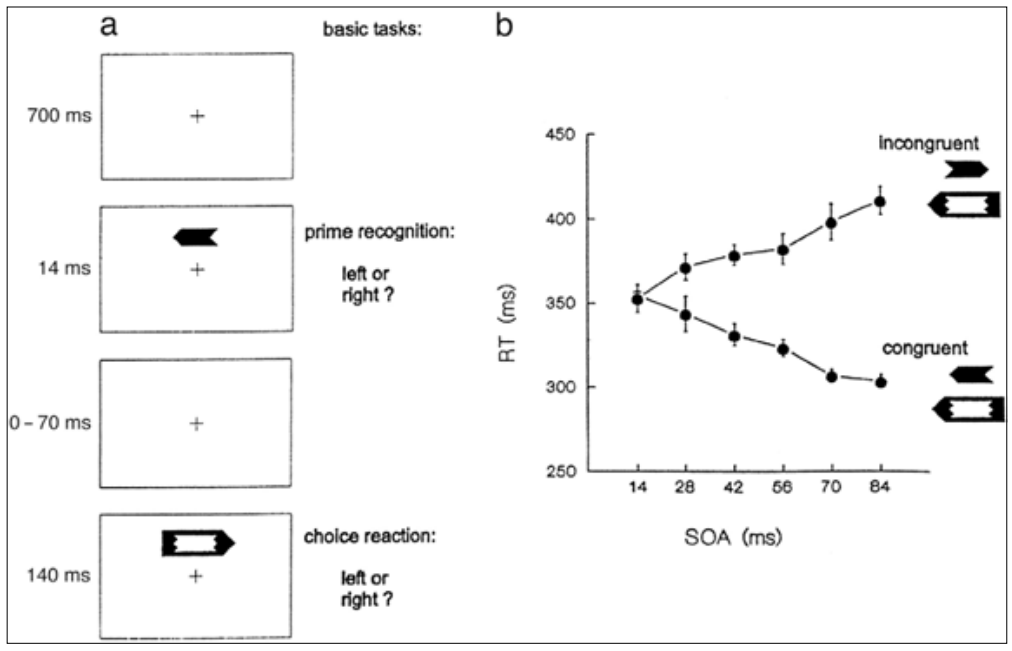

Abb. 15: ,Unbewusste Wahrnehmung‘. Obwohl die Richtung des ersten Pfeiles nicht erkannt wird, beeinflusst sie die Reaktionszeit auf die Darbietung des zweiten Pfeiles. ${ }^{18}$

Dieses Experiment zeigt, dass es in der Tat unbewusste Wahrnehmung gibt. Wir nehmen nicht alles wahr, was wir sehen, und was unser Handeln beeinflusst. Ein besonders eindrückliches Beispiel ist Yoram Bonneh's Täuschung (Motion-Induced Blindness), die unter http://www. weizmann.ac.il/home/masagi/MIB/mib-basic.html beobachtet werden kann. Abb. 16 zeigt eine stationäre Version dieses bewegten Reizes. Er umfasst eine Wolke aus kleinen blauen Punkten, die sich bewegen und dabei den Eindruck einer Kugel erzeugen. Zusätzlich werden 3 größere, helle gelbe Punkte dargeboten. Während der Betrachtung des Reizes wird die Aufmerksamkeit des Beobachters automatisch auf die Kugel gezogen, die durch die blauen Punkte definiert ist. Nach kurzer Beobachtungszeit verschwinden für die meisten Beobachter einer oder mehrere der gelben Punkte für kurze Zeit. Das Phänomen ist insbesondere deshalb frappierend, weil die gelben Punkte einen höheren Kontrast aufweisen als die blauen Punkte, aber dennoch zeitweise unterdrückt werden. Auch dieses Phänomen demonstriert, dass die cortikale Repräsentation der Wirklichkeit von der tatsächlichen Wirklichkeit deutlich abweichen kann. Es ergeben sich hieraus auch Komplikationen für die Rechtswis-

18 Vorberg, D. et al.: „Different time courses for visual perception and action priming“, in: Proceedings of the National Academy of Sciences, 100 (2003), S. 6275-6280, Abb. 1. 
senschaft, insbesondere für die generelle Glaubwürdigkeit der Aussagen von so genannten Augenzeugen. Doch hiermit sei es genug der Fehlschläge der Konstruktion von Wirklichkeit in unserem Gehirn.

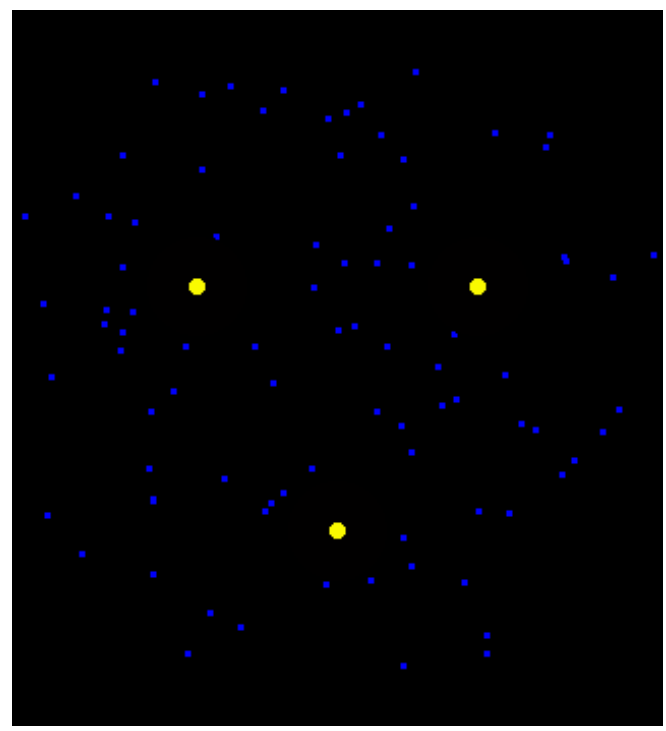

Abb. 16: Bonneh's Illusion (Motion-Induced Blindness).

\section{Kriterien der Konstruktion von Wahrnehmung}

Ähnlich wie bei der Beschreibung der Verarbeitungskriterien im Cortex, die oben angegeben wurden (wie Spezialisierung, Parallelverarbeitung, laterale und vertikale Integration), können auch Kriterien für die Konstruktionsprinzipien der Wahrnehmung angegeben werden. Es seien hier drei Kriterien vorgestellt: Vertrautheit, Ordnung und Ästhetik. Ästhetik könnte demnach eine wesentliche Rolle bei der Erzeugung cortikaler Repräsentation von Wirklichkeit spielen. Dabei soll im folgenden Ästhetik verkürzt als „Schönheit“" verstanden werden einschließlich aller Spezifikationen von „Erregung“ bis zur „Neuheit“. Leist ${ }^{19}$ deutet auf eine Beziehung zwischen der Biologie einerseits und der Geisteswissenschaft (deren Domäne die Ästhetik ist) andererseits hin, indem er schreibt, dass Schönheit der implizierte symbolische Ausdruck geistiger und physikalischer Gesundheit sei (,implicit symbolic expression of mental and physi-

19 Leist, A.: „What Makes Bodies Beautiful“, in: Journal of Medicine and Philosophy, 28, 2 (2003), S. 187-219. 
cal health"). Im Laufe der Tagung wurde ja bereits darauf hingewiesen, dass physische Schönheit als Ausdruck von Gesundheit aufgefasst werden kann. Es scheint unmittelbar einleuchtend, dass sich auch die geistige Gesundheit bis zu einem gewissen Grade in den Zügen und der Mimik eines Menschen widerspiegeln kann und insofern physische Schönheit in bestimmten Grenzen auch Hinweise auf die mentale Gesundheit geben kann. Doch dieser Aussage wird auch hin und wieder widersprochen.

Bei der Beurteilung physischer Schönheit spielt die Farbe der Zähne allerdings praktisch keine Rolle, wie amerikanische Zahnärzte nachweisen konnten. Dort spielt das Aufhellen der Zähne eine wesentlich größere Rolle als in Europa, scheint aber bei der Beurteilung der Attraktivität von Menschen keinen nennenswerten Vorteil $\mathrm{zu}$ erbringen. ${ }^{20}$ Dagegen weist Symmetrie eine hohe Korrelation mit Schönheitsaussagen auf. In Abb. 17 werden regelmäßig die Reize, die symmetrisch sind, als attraktiver eingeschätzt als diejenigen, die asymmetrisch sind.

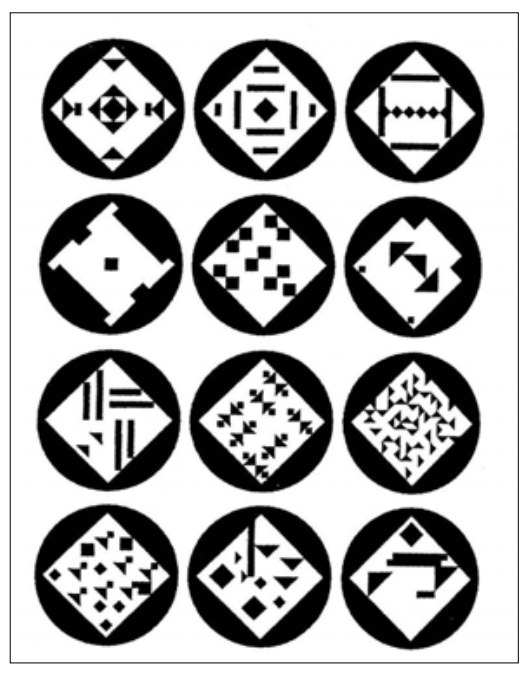

Abb. 17: Symmetrische Reize (oberer Teil der Abbildung) werden in der Regel als ,ästhetischer' beurteilt als asymmetrische Reize (unterer Teil der Abbildung) ${ }^{21}$.

20 Grosofsky, A. et al.: „Tooth Color: Effects on judgments of attractiveness and age" in: Perceptual and Motor Skills, 96 (2003), S. 43-48.

21 Jacobsen, T./Höfel, L.: „Aesthetic judgments of novel graphic patterns: Analysis of individual judgments", in: Perceptual and Motor Skills, 95 (2002), S. 755-766, hier S. 759, Abb. 1. 
Wir hatten uns im Rahmen der Tagung darauf geeinigt, dass ein Gesicht (mehr oder weniger) das „ehrliche Signal“ sowohl der genotypischen als auch der phänotypischen Qualität des Besitzers dieses Gesichtes sei. ${ }^{22}$ Setzt man eine positive ästhetische Einschätzung mit Attraktion, also Anziehungskraft des entsprechenden Objektes gleich, dann ist die Aussage richtig, dass eine generelle Attraktion für prototypische Exemplare besteht (,a general attraction to prototypical exemplars “). Es ist seit einigen Jahren bekannt, dass Menschen generell dazu neigen, durchschnittliche Gesichter, d.h. solche, die durch eine Mittelwertsbildung über viele Beispiele gewonnen wurden, als schön oder zumindest als relativ attraktiv zu empfinden. Wie neuere Ergebnisse beweisen, gilt das gleiche für Hunde, Vögel und sogar Armbanduhren. ${ }^{23}$ Auch hier werden diejenigen Darstellungen als besonders anziehend eingestuft, die durch Mittelwertsbildung über viele individuelle Objekte entstehen. Die Interpretation ist keinesfalls trivial. Im Falle von Gesichtern könnte argumentiert werden, dass jede Abweichung von der Norm als Hinweis auf eine genotypische oder phänotypische Abweichung, d.h. auf einen genetischen Defekt oder auf eine Krankheit, aufgefasst werden kann. Solche Abweichungen sind bei der Partnerwahl sehr wichtig, da das Genom eines Individuums darum bemüht ist, möglichst gesunde und attraktive Nachkommen als Träger des Genoms zu erzeugen (um eine provokative Formulierung zu wählen). Dieses Problem der Auswahl des best geeigneten Geschlechtspartners stellt sich natürlich nicht, wenn Menschen Vögel oder Hunde beurteilen, und schon gar nicht bei Armbanduhren. Das bedeutet, dass die Präferenz der durch Mittelwertsbildung erzeugten Individuen oder Objekte nicht rein auf Kriterien der Partnerwahl beruhen kann. Die Lösung dieses Problems wird weitere Forschungsanstrengungen erfordern.

Doch zurück zu den Gesichtern. Wie werden Gesichter im Cortex des Menschen repräsentiert? Es ist seit langem klar, dass ein umschriebener Bereich des Cortex existieren muss, der insbesondere auf die Analyse von Gesichtern spezialisiert ist. Denn eine ganze Reihe von Patienten sind nach Infarkten des Cortex nicht mehr in der Lage, Gesichter zu unterscheiden und können daher ihre Mitmenschen visuell nicht unterscheiden, solange sie nicht besondere Auffälligkeiten wie einen Bart,

22 Senior, C.: „Beauty in the Brain of the Beholder“, in: Neuron, 38 (2003), S. 525-528.

23 Halberstadt, J./Rhodes, G.: „The attractiveness of nonface averages: Implications for an evolutionary explanation of the attractiveness of average faces“, in: Psychological Science, 11, 4 (2000), S. 285-289. 
eine ausgefallene Brille oder eine besondere Haartracht aufweisen. Das entsprechende Krankheitsbild, Prosopagnosie, lässt die übrigen visuellen Wahrnehmungsleistungen weitgehend unbeeinträchtigt. Dieser Befund zeigt, dass Gesichter für den Menschen als sozial lebendes Wesen sehr wichtig sind. Einzelne Mitglieder der Gruppe werden primär aufgrund ihres Gesichtes voneinander unterschieden, Gemütszustände und Launen werden erkannt und Geschlechtspartner ausgewählt.

Bei der Untersuchung der entsprechenden Hirnareale des Rhesusaffen fanden sich viele Nervenzellen, die spezifisch auf Gesichter reagierten, d.h. ihre Entladungsfrequenz bei Darbietung von Gesichtern erhöhten. Abb. 18 zeigt die Ergebnisse der Untersuchung eines solchen Neurons. Das Neuron erhöht seine Entladungsrate insbesondere dann sehr stark, wenn das Bild eines anderen Affen dargeboten wird. Erstaunlicherweise reagiert die Zelle auf eine Art von Cartoon eines Gesichtes fast ebenso stark, wie in Abb. 18b gezeigt wird. Obwohl also eine extreme Verarmung der visuellen Information beim Übergang von $18 \mathrm{a}$ nach $18 \mathrm{~b}$ stattgefunden hat, reagiert die Zelle fast ebenso stark auf diesen Cartoon eines Gesichtes wie auf ein echtes Gesicht. Ähnlich ergeht es auch dem menschlichen Betrachter und wir finden hier wieder das Phänomen, dass Menschen auf Cartoons/Zeichnungen sehr stark ansprechen. Neu ist, dass offenbar das gleiche für den Cortex des Rhesusaffen gilt. Fehlt dagegen im Reiz zusätzlich der Mund, reagiert die Zelle kaum noch. Wird nur der Mund dargeboten, dann bleibt die Zelle ebenfalls inaktiv. Auch ein Fehlen der Außenkontur des Gesichtes führt zum Ausbleiben der Reaktion; das gleiche gilt für die reine Darbietung der Außenkontur. Die zusätzliche Darbietung eines Rechteckes außerhalb des Gesichtes stört nicht. Dagegen führt die Umkehrung des Kontrastes von Augen und Mund dazu, dass diese Figur von dem Neuron nicht mehr als Gesicht akzeptiert wird und die Zelle nicht länger reagiert. Das ist vermutlich der Grund dafür, dass schon immer bei Halloween und heute in manchen Monsterfilmen durch Aufhellen der Augen ein starker Verfremdungseffekt erzeugt werden kann. Im Falle des Neurons im Affencortex stören massive Änderungen des Reizes, insbesondere der Übergang von einem echten Gesicht zu einer Strichzeichnung, das Antwortverhalten kaum, aber der relativ kleine Schritt durch Kontrastumkehr von Auge und Mund zerstört die Antwort vollständig. 


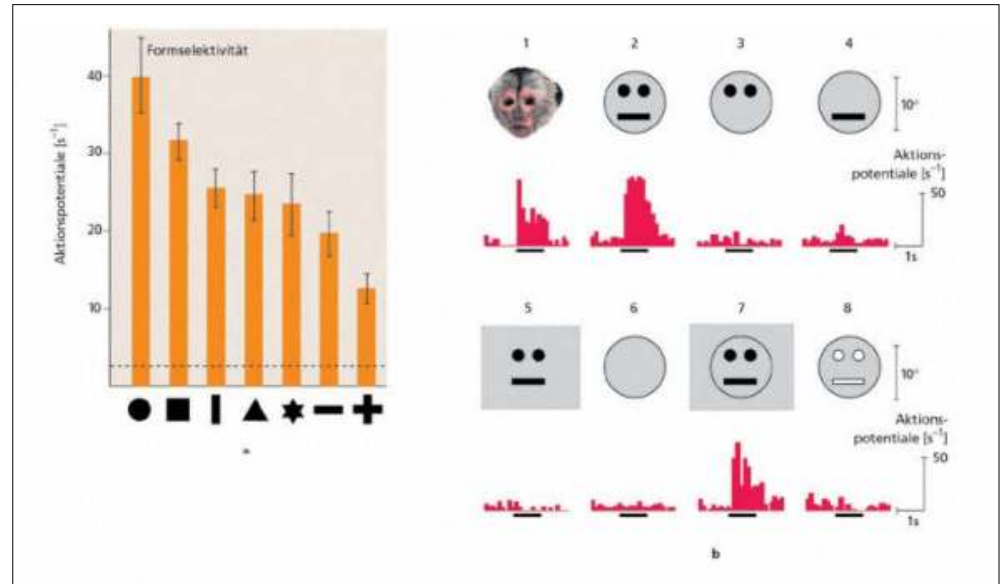

Abb. 18: Beispiel einer Nervenzelle im Cortex eines Affen, die ihre Entladungsfrequenz stark erhöht, wenn das Foto eines Affenkopfes präsentiert wird. Die Zelle reagiert ebenso gut auf den,Cartoon 'eines Gesichtes, nicht aber auf einen Cartoon, bei dem zusätzlich eine Kontrastumkehr von Auge und Mund stattgefunden hat. ${ }^{24}$

Eine weitere Möglichkeit, Gesichter zu verfremden, besteht darin, sie auf dem Kopf stehend darzustellen. Die Abb. 19 erscheint relativ unauffällig. Wenn Sie nun aber die Seite um $180^{\circ}$ drehen, werden Sie sehen, dass hier nicht etwa Margret Thatcher einfach nur auf dem Kopf stehend abgebildet wurde. Man sieht, dass es bei einem auf dem Kopf stehenden Gesicht nicht weiter stört, wenn Mund und Augen nicht zum Ganzen passen. ${ }^{25}$ Dagegen wirkt es bei einem Gesicht, das aufrecht, also in der gewohnten Ausrichtung, dargeboten wird, massiv entstellend, wenn isoliert die Augen und der Mund um $180^{\circ}$ gedreht dargeboten werden.

24 Kandel/Schwartz/Jessel (wie Anm. 2), S. 565-566, Abb. 28.17 u. 28.18.

25 Thompson, P.: „Margaret Thatcher: a new illusion“, in: Perception, 9, 4 (1980), S. 483-484. 


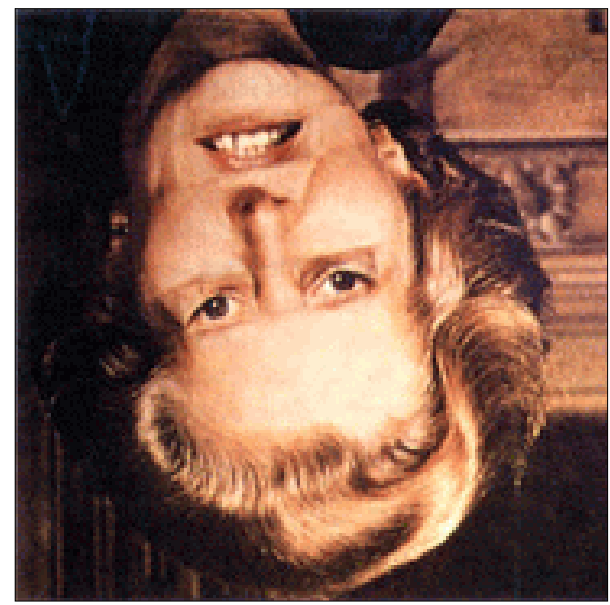

Abb. 19: Maggie Thatcher steht auf dem Kopf. Fällt Ihnen etwas auf? Drehen Sie die Seite doch so, dass Frau Thatcher nicht mehr auf dem Kopf steht! ? $^{26}$

Symmetrie ist eine Eigenschaft von Objekten, die eher zu einem positiven ästhetischen Urteil führt. Das gilt beispielsweise für Gesichter, denn durch die Mittelwertsbildung über viele Gesichter, wie sie oben beschrieben wurde, werden u.a. die Asymmetrien beseitigt, die in jedem Gesicht mehr oder weniger stark ausgeprägt vorhanden sind. Auch unter geometrischen Mustern, wie sie in Abb. 17 dargestellt sind, werden in der Regel die symmetrischen Reize, die sich im oberen Teil der Abbildung befinden, als attraktiver beurteilt, als die asymmetrischen Reize, die im unteren Teil wiedergegeben sind. Die ausgewogene Verteilung von Objekten in einem Zen-Garten wird in der Regel ebenfalls als ästhetisch empfunden und es ist vor einigen Jahren auch gelungen, einen Algorithmus zu finden, der die als ,schön und harmonisch“ empfundene Anordnung der Gegenstände in diesem Zen-Garten beschreibt. Auch die mehrfache Wiederholung eines Objektes oder Themas kann u.U. als schön empfunden werden, oder aber auch als beunruhigend oder als langweilig, je nach Betrachter. Diese Wiederholung könnte im positiven Fall zu einer Art von Resonanz oder harmonischer Schwingung der elektrischen Erregung im Gehirn führen, aber das ist Spekulation.

26 Thompson (wie Anm. 24), S. 483, Abb. 1. 


\section{Spekulative Folgerungen und Ausblick}

Was zeichnet also ästhetische Objekte aus? Man könnte die Aussage wagen, dass sie in der Regel anziehend wirken, also attraktiv sind, dass sie eher angenehme Gefühle erzeugen und dass diese eine bestimmte Mindest-Stärke aufweisen müssen. Das gilt m.E. auch für Kunstobjekte der letzten Dekaden, die sicher nicht immer im engeren Sinne als „schön“ bezeichnet werden können. Dennoch üben sie eine gewisse Faszination aus, die möglicherweise damit zusammenhängt, dass Emotionen induziert werden - auch wenn diese vielleicht zunächst eher nicht lustvoller Natur sind. Könnte es sein, dass es dem modernen Menschen ausreicht, überhaupt Emotionen zu empfinden, die durch ein Kunstwerk erzeugt werden, um diesem Kunstwerk eine ästhetische Qualität abgewinnen zu können? Sicherlich werden Objekte, die (im weiteren Sinne) als ästhetisch bezeichnet werden, insgesamt höher bewertet als andere Objekte. Und sicherlich signalisieren ästhetische Objekte in der Regel „Qualität“, was immer darunter im Einzelnen zu verstehen sei. Bei Lebewesen ist das ästhetische Urteil häufig mit Eigenschaften wie Gesundheit, Stärke und Modellhaftigkeit assoziiert. Ästhetische Objekte sind, wie im Rahmen der Tagung betont wurde, oft teuer, denn sie kosten Ressourcen, Vitalität und/oder Zeit. Und irgendwie sind ästhetische Objekte lustbetont. Sie erzeugen im Beobachter ein Gefühl, das er oder sie im Endeffekt positiv beurteilt und nach Möglichkeit zu wiederholen trachtet. Ästhetische Objekte könnten daher in der Lage sein, das dem Menschen intrinsische Belohnungs- und Erregungssystem zu aktivieren. Menschen besitzen dieses System, zu dem im Gehirn beispielsweise das limbische System und die Amygdala in beiden Hirnhälften gehören. Dieses System könnte an der Vermittlung des ästhetischen Urteils bzw. der damit verbundenen subjektiven Empfindungen beteiligt sein.

Ich könnte mir auch vorstellen, dass ästhetische Urteile teilweise davon abhängen, inwieweit dargebotener Reiz und Erwartungshaltung zusammenpassen, inwieweit also ,bottom-up“ und „top-down“-Signale übereinstimmen (schließlich besteht das Wesen vieler Witze darin, dass diese beiden Signalquellen nicht übereinstimmen). Offensichtlich existieren also in der Außenwelt bestimmte Reize, die in der Lage sind, ein dem Menschen inhärentes Bewertungssystem zu aktivieren, das seinerseits mit subjektiv empfundenen positiven Gefühlen bzw. „Lust“ im weiteren Sinne gekoppelt ist. Diese Auslöser sind teilweise sicherlich phylogenetisch erworben, also in unserem Genom niedergelegt, teilweise aber auch durch die persönliche Geschichte in der jeweiligen Gesellschaft bedingt. 
Natur (genauer: Genom) und Umwelt sind hier aufs engste miteinander verbunden, wenn es um ästhetische Urteile geht. Ich würde annehmen, dass der Einfluss der phylogenetisch codierten Auslöser stärker ausgeprägt ist bei ästhetischen Urteilen, die sich auf den Körper von möglichen Sexualpartnern beziehen, so wie überhaupt auf den Erhalt des eigenen Genoms, also beispielsweise das Ansprechen auf Babys. Auch die Beurteilung von Nahrung - „das Auge isst mit“ - beinhaltet eine ästhetische Komponente, die sicherlich teilweise genetisch codiert ist. Das unmittelbare Verstehen von Emotionen aufgrund der Mimik von Mitmenschen könnte ebenfalls zu einem großen Teil genetisch codiert werden, da viele Komponenten der Mimik relativ unverändert bei allen Kulturen und Rassen zu finden sind. Als sozial lebendes Wesen ist der Mensch, wie oben bereits ausgeführt, wesentlich darauf angewiesen, sich den anderen Mitgliedern der Gemeinschaft gegenüber situationsgerecht zu verhalten und dazu ist es wichtig, die jeweilige Gestimmtheit der Anderen möglichst rasch und genau zu erkennen (und die eigene kundzutun). Ich würde soweit gehen zu behaupten, dass auch Begriffe wie Sympathie und Antipathie mit ästhetischen Urteilen zusammenhängen, zumindest entfernt. Jedenfalls können ästhetische Urteile emotionale Reaktionen hervorrufen und daher kann die Darstellung bzw. das Schenken positiv ästhetisch besetzter Objekte (Blumen, Schmuck) die Stimmungslage positiv beeinflussen. Man könnte in diesem Sinne sagen, dass Ästhetik auch etwas mit Stimmungs-,Manipulation' zu tun hat und mit dem Erreichen ähnlicher Gestimmtheiten bei unterschiedlichen Mitgliedern einer Gruppe.

Auch das Habitat, also das Umfeld, in dem der Mensch lebt, ist für ihn wichtig. Insofern könnten auch in dieser Hinsicht Präferenzen durch das Genom eingebaut sein, die sich als ästhetische Urteile manifestieren. All diesen teilweise durch das Genom codierten „Langzeitadaptationen“ stehen die Kurzzeitadaptationen gegenüber. Denn die Welt verändert sich schnell und ein Lebewesen wie der Mensch, das über eine relativ lange Lebensspanne verfügt, muss sich dieser Welt während der persönlichen Lebensgeschichte anpassen, da die Generationenfolge für eine zufällige Modifikation des Genoms und anschließende Selektion viel zu lang ist. Die Instinktentbundenheit, die einhergeht mit der enormen Vergrößerung des Großhirns beim Menschen erlaubt es der Gattung Homo Sapiens, eine unglaubliche Menge an Informationen über die Welt individuell zu speichern und auf diese Weise die im Genom kodifizierte Information über die Welt zu ergänzen und rasch anzupassen. Insofern sind fast alle menschlichen Handlungen sowohl durch das Genom als auch durch die 
physikalische und soziale Umwelt beeinflusst. Lernen spielt also eine große Rolle. Ich habe mich in den letzten Jahren stark mit perzeptuellem Lernen beschäftigt, d.h. ich habe versucht, die Mechanismen, die beim Training von visuellen Wahrnehmungsaufgaben auftreten, besser zu verstehen. Dabei stellte sich heraus, dass perzeptuelles Lernen die Verarbeitung visueller Signale bereits auf einer sehr frühen Stufe verändern kann. In Abb. 20 sehen Sie die Ergebnisse von 12 Versuchspersonen, die angeben sollten, ob der obere von zwei Strichen relativ zum unteren nach rechts oder nach links versetzt war. Entsprechend lautete die Aufgabe bei horizontal orientierten Strichen zu entscheiden, ob der rechte nach oben oder nach unter versetzt war. Die Prozentzahl richtiger Antworten für eine relativ geringe Versetzung zwischen den beiden Linien verbesserte sich innerhalb von 20 Minuten Trainings von etwa 75 auf fast $90 \%$. Drehte man die Reize dann um $90^{\circ}$, mussten die Versuchspersonen vollständig neu lernen, d.h. der Lernerfolg konnte nicht auf die neue Reizorientierung generalisiert werden. Daraus kann man schließen, dass diesem perzeptuellen Lernen keine im engeren Sinne kognitive Verbesserung der visuellen Informationsverarbeitung zugrunde liegt, da man bei einer kognitiven Leistung einen Transfer des Erlernten auf eine neue Orientierung erwarten müsste. Dieser Folgerung entspricht, dass der Lernerfolg auch nicht zwischen den beiden Augen transferiert. Wird also die Aufgabe gelernt, während ein Auge mit einer Augenklappe abgedeckt ist, dann muss die Versuchsperson die gleiche Aufgabe erneut erlernen, sobald die Augenklappe auf das zuvor geöffnete Auge transferiert wird.

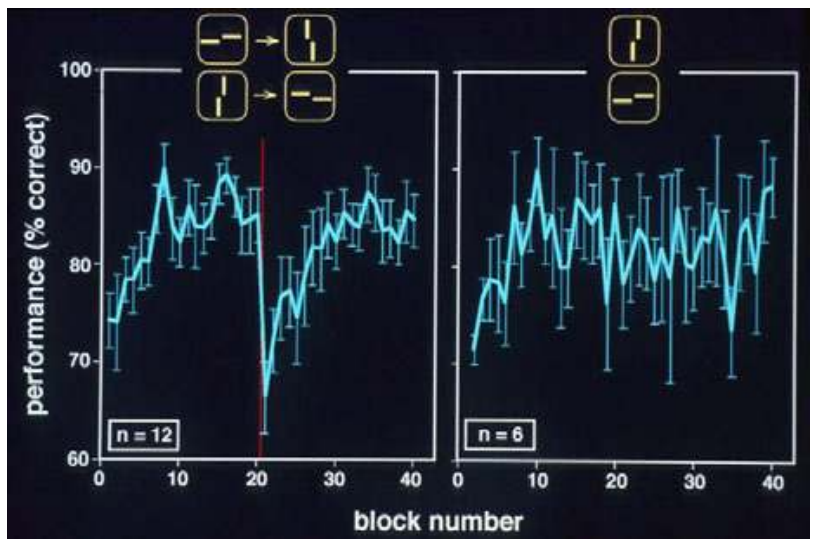

Abb. 20: Anstieg der Prozentzahl richtiger Antworten durch kurz dauerndes Training einer Strich-Unterscheidungsaufgabe durch perzeptuelles Lernen und Abfall der Leistung nach Drehung des 
Testreizes. Linke Seite: Nach Drehung des Reizes um $90^{\circ}$ fällt die Leistung der Versuchspersonen stark ab. Rechte Seite: kein Abfall der Leistung für konstante Reizorientierung. ${ }^{27}$

Diese Ergebnisse zeigen, dass sich die Analyse der Außenwelt durch die Auseinandersetzung mit dieser Außenwelt und durch Lernen bereits auf sehr frühen cortikalen Verarbeitungsebenen verändert. Durch das Lernen wird das System, welches die Außenwelt analysiert, selbst verändert; die Welt wird nicht nur anders interpretiert, sie wird auch teilweise anders synthetisiert. Auf diese Art und Weise können die Reize, die durch die Sinnesorgane aufgenommen werden, im zentralen Nervensystem modifiziert werden und es kann eine Umdeutung stattfinden. Durch die persönliche Lebensgeschichte kann die Bedeutung so genannter angeborener Auslöser verändert werden, ästhetische Bewertungen können modifiziert werden und Reize, für die vom Genom keine Aktivierung des Belohnungssystems vorgesehen worden war, können mit diesem System assoziiert werden. D.h. die Kultur, die Medien, die Umwelt sind in der Lage, innerhalb gewisser Grenzen ästhetische Urteile zu modifizieren und, zumindest für die junge Generation, neu zu prägen. Die geprägten, also frühkindlich erlernten Auslöser sind sicherlich die stabilsten, wodurch es zu tiefgreifenden Unterschieden zwischen den Urteilen und Empfindungen unterschiedlicher Generationen kommen kann und damit der Weg frei gemacht wird für die Akzeptanz von Medienumbrüchen.

Eine weitere, deutlich unterschiedliche Klasse ästhetischer Reize könnte, wie oben angedeutet, auf Faktoren wie Komplexität, Spannung und Überraschung beruhen. Der Mensch als „bestia cupidissima rerum novarum" kann Lust beim Erleben neuer, komplexer und unerwarteter Reize erleben und aufgrund dieser Lustempfindung den auslösenden Objekten ästhetische Qualitäten zuschreiben. Wenn man die beiden Prinzipien des Lernens einerseits und der Spannung und Überraschung andererseits miteinander kombiniert, dann lässt sich auch die Antiästhetik in einer Biologie der Ästhetik unterbringen, da durch Lernen neue, eigentlich nicht ästhetische Objekte im konventionellen Sinne an das „Lustsystem" angekoppelt werden können. Diese Assoziation kann überdies durch Prädikate wie teuer und wertvoll unterstützt werden. Eine dritte Ursache für positive ästhetische Urteile im Bereich der visuellen Wahrnehmung könnte durch eine Art von Resonanz bestimmter Bereiche un-

27 Poggio, T./Fahle, M./Edelman, Shimon: „Fast perceptual learning in visual hyperacuity“, in Science, 256 (1992), S. 1018-1021, hier S. 1020, Abb. 4. 
seres Cortex bedingt sein. Prinzipien wie Symmetrie und Ordnung, Proportionen und goldener Schnitt, gute Gestalt und ähnliches könnten zu Resonanzen der elektrischen Hirnaktivität führen.

In Fortführung dieser Spekulation könnte man fragen, ob vielleicht in dieser Hinsicht auch Beziehungen zur Musik existieren, also zu Begriffen wie Harmonie. Es ist mittlerweile bekannt, dass aufgrund der lateralen Interaktionen, die oben angesprochen wurden, benachbarte Areale und Bereiche des Cortex miteinander interagieren. Unter bestimmten Umständen treten so genannte Erregungswellen auf, die sich langsam über die Oberfläche des Großhirns ausbreiten. Es wäre denkbar, dass eine geeignete zeitliche und räumliche Anordnung von Reizen dazu führt, dass solche Erregungswellen ausgelöst werden, die durch die Außenreize weiter verstärkt werden - sei es durch Musik oder durch bestimmte Reizeigenschaften visueller Muster wie (die oben genannten) Symmetrie, Ordnung, Proportionen, goldener Schnitt und gute Gestalt. Bei diesen Überlegungen handelt es sich natürlich um wildeste Spekulationen, die jedoch prinzipiell der naturwissenschaftlichen Analyse, beispielsweise mit Hilfe der funktionellen Kernspintomographie, zugänglich sind. Und insofern sollte ein Naturwissenschaftler an dieser Stelle schlieBen, mit der Hoffnung, in Zukunft zu diesen Fragen neurobiologische Aspekte beitragen zu können.

\section{Zusammenfassung}

Alle Informationen, auf denen die Repräsentation der visuellen Außenwelt in unserem Gehirn beruht, müssen zunächst im Auge aufgenommen werden. Die riesige Menge von Signalen, die vom Auge an das Gehirn weitergeleitet wird, muss zunächst durch Elimination von Redundanzen reduziert werden. Das Bild der Außenwelt wird auf eine Art von „Cartoon"-Zeichnung reduziert und diese wird parallel und gleichzeitig in einer ganzen Reihe von spezialisierten Cortexarealen verarbeitet, meist simultan für verschiedene Bereiche des Gesichtsfeldes. Auf diese Weise wird im Cortex durch die Zusammenarbeit von Signalen aus den Sinnesorganen („bottom-up“) und von Vorwissen und Erwartungshaltungen (,top-down“) eine Repräsentation der Außenwelt erstellt, die sowohl Analyse als auch Synthese darstellt. Diese cortikale Repräsentation der Außenwelt ist individuell unterschiedlich, da sich sowohl die Signale aus den Sinnesorganen bei unterschiedlichen Individuen unterscheiden können als auch die Vorinformationen und Erwartungshaltungen, so dass gleiche Reize bei unterschiedlichen Individuen zu merklich verschiede- 
nen Erregungszuständen und mithin unterschiedlichen Repräsentationen führen können. Nur durch die Verwendung einer gemeinsamen Sprache, die den unterschiedlichen Erregungsmustern individueller Gehirne die gleichen Worte zuordnet, werden diese Differenzen weitgehend kaschiert. Wahrnehmungstäuschungen stellen überzeugende Beispiele für das Fehlschlagen dieser Rekonstruktion und Repräsentation der Außenwelt in unserem Gehirn dar: Bei diesen Reizen schlägt die korrekte Rekonstruktion des dargebotenen Objektes im Gehirn fehl. Unter den „topdown"-Einflüssen, also den Erwartungshaltungen und Konstruktionsprinzipien, auf denen die Synthese der Repräsentation beruht, könnte auch die Ästhetik im weiteren Sinne einen Platz einnehmen. 



\section{SIEGFRIED FREY}

\section{DAS BILD VOM ANDERN: FUNKTIONSPRINZIPIEN DER VISUELLEN E IN D R U C KS B I L D U N G ${ }^{1}$}

Die Welt, die wir aus eigener Erfahrung kennen, ist im Grunde winzig klein. Jeder von uns lebt und arbeitet bekanntlich auf einem ganz schmalen Teil der Erdoberfläche, bewegt sich innerhalb eines kleinen Kreises von Personen und auch von diesen Bekannten kennt er wiederum nur ganz wenige wirklich genau. Dessen ungeachtet tendieren wir nun aber alle dazu, Meinungen zu entwickeln, die sich über einen weit größeren Raum, eine viel größere Zeitspanne, eine größere Anzahl von Personen erstrecken als für uns direkt überschaubar ist.

Dass es sich bei diesen Meinungen in der Regel nicht etwa um Wahnvorstellungen handelt, sondern um solides Wissen, auf das wir uns in unserem Denken und Handeln getrost verlassen können, ist nicht primär unser eigenes Verdienst. Wir verdanken die Chance zu einer nahezu grenzenlosen Erweiterung unseres engen persönlichen Erfahrungshorizontes vielmehr einzig dem Umstand, dass sich unsere Spezies, als einzige auf diesem Planeten, mit der Erfindung der Sprache ein eigenes Kommunikationswerkzeug geschaffen hat. Denn erst vermittels der Sprache eröffnet sich dem menschlichen Individuum die Möglichkeit, das Wissen, die Ideen und die Wertvorstellungen seiner sozialen Umwelt kennen zu lernen und für die Zwecke seiner eigenen Lebensführung nutzbar zu machen.

Der Versuch, aus dieser reichen Informationsquelle nutzbringendes Wissen zu schöpfen, stellt den Einzelnen nun allerdings vor eine äußerst schwierige Aufgabe. Denn die aus dem sozialen Umfeld mündlich oder schriftlich angelieferten Informationen tragen ja in der Regel keineswegs das Gütesiegel absoluter Verlässlichkeit. Sie stehen vielmehr üblicherweise in Konkurrenz zu ganz anders lautenden Aussagen, die andere Personen über denselben Sachverhalt machen. Aus diesem Grund ist der Einzelne denn auch praktisch permanent mit dem Problem konfrontiert,

1 In memoriam Prof. Dr. Karl-Josef Frey 
sich darüber klar werden zu müssen, ob er der Mitteilung eines Informanten Glauben schenken oder ihr misstrauen will, ob er dessen Meinung beipflichten oder ihr widersprechen, dessen Handlungsvorschlag zustimmen oder ihn ablehnen soll.

Die zeitlichen, materiellen und personellen Ressourcen, die zur Beschaffung der Information erforderlich wären, die zur Überprüfung der Richtigkeit einer Aussage bzw. zu einer umfassenden Auseinandersetzung mit dem Für und Wider unterschiedlicher Positionen benötigt würden, stehen dem Individuum naturgemäß nur in den seltensten Ausnahmefällen zu Gebote. Insoweit es nun aber für die Einzelperson illusorisch ist, eine zufrieden stellende Abklärung des in Frage stehenden Sachverhalts aus eigener Kraft herbeizuführen, bleibt ihr im Hinblick auf die Frage, ob sie eine Aussage als richtig erachten bzw. ob sie sich eine bestimmte Position zu eigen machen will, gewissermaßen nur noch die Option zu entscheiden, ob sie den Informanten als glaubwürdig einstufen will bzw. ob und in wieweit sie bereit ist, der Person, die diese Position vertritt, ihr Vertrauen entgegenzubringen.

Die Aufgabe, sein jeweiliges Gegenüber zu taxieren, stellt sich dem Individuum umso dringlicher, als ein Großteil der sprachlichen Äußerungen, die von unserem sozialen Umfeld tagtäglich erzeugt werden, ja nicht etwa eigens von uns erbeten wurden, sondern ganz unaufgefordert an uns herangetragen werden. Es ist deshalb auch keineswegs gewährleistet, dass diese Mitteilungen in jedem Falle durch die uneigennützige Intention des Gesprächspartners motiviert sind, uns an dessen ,reichen Wissensschatz' teilhaben zu lassen. Vielmehr ist durchaus denkbar, dass der Informant in vielen Fällen durch die auf sprachlichem Wege verbreitete Information in Wahrheit lediglich seinen eigenen Interessen zu dienen versucht. Da nun aber die Mitglieder einer Sprachgemeinschaft ein vitales Interesse daran haben müssen zu verhindern, dass sie gegen ihren Willen für die eigennützigen Zwecke des Sprechers eingespannt und ausgenutzt werden, stellt die Entwicklung eines Referenzsystems, mit dessen Hilfe sich ein höchst nuanciertes ,Bild vom Andern' zeichnen lässt, eine gesellschaftliche Aufgabe ersten Ranges dar.

\section{Achtzehntausend Eigenschaftsbegriffe}

Dass unsere Spezies sich der Herausforderung, ein Vokabular zur sprachlichen Fixierung individueller Unterschiede zu schaffen, nicht nur stellte, sondern über ihre ganze Entwicklungsgeschichte hinweg darum bemüht war, dieses Vokabular fortwährend zu erweitern, wurde erstmals 
in den 30er Jahren des vergangenen Jahrhunderts in voller Deutlichkeit ersichtlich. In dem Bemühen, der damals gerade erst im Entstehen begriffenen Disziplin der Persönlichkeitspsychologie eine wissenschaftliche Grundlage zu verschaffen, stellten sich Henry S. Odbert vom Dartmouth College, der amerikanischen „Ivy League“ Universität, die Jahre später eine legendäre Rolle auch bei der Entstehung der Computernetze spielen würde, sowie sein Lehrer Gordon W. Allport von der Harvard Universität die Aufgabe, eine Liste sämtlicher Eigenschaftsbegriffe zu erstellen, die zur Beschreibung von Personen verwendet werden können.

Als Ausgangsbasis für diesen bis heute unübertroffenen Versuch, den gesamten Umfang des Vokabulars zur Charakterisierung individueller Unterschiede zu ermitteln, diente ihnen Webster's New International Dictionary in der Ausgabe von 1926. In einer minutiösen Durchsicht der dort verzeichneten, rund 400.000 Termini erarbeiteten die Autoren eine Liste sämtlicher Adjektive, die die englische Sprache für die Benennung von Eigenschaften bereithält. Das Ergebnis dieser Recherche machte erstmals auf den erstaunlich großen Umfang des Vokabulars zur Beschreibung menschlicher Wesenszüge aufmerksam. Die unter dem Titel „Trait-Names. A Psycho-lexical Study“ im Jahre 1936 publizierte Adjektivliste umfasste nicht weniger als 17.953 Worte oder $4 \frac{1}{2}$ Prozent des gesamten englischen Sprachschatzes. Sie überstieg damit um das nahezu 18 fache eine damals weithin akzeptierte Schätzung, zu der ein halbes Jahrhundert zuvor Darwins Vetter Francis Galton ${ }^{2}$ in seiner Studie „Measurement of Character" gelangt war.

Zur Entstehung dieses gewaltigen Repertoires an Eigenschaftsbegriffen hatte, wie aus der von Allport und Odbert zusammengestellten Liste klar hervorging, der über alle Epochen unserer Kulturgeschichte hinweg nachweisbare Hang beigetragen, das Gegenüber im Lichte der Wertvorstellungen und Interessen zu beurteilen, die während des jeweiligen Zeitalters als besonders wichtig erachtet wurden. Dispositionen wie „devotion, pity, and patience“, existierten, wie die Autoren zurecht geltend machten, möglicherweise ,im Innern' einzelner Individuen vielleicht schon seit Urzeiten, „but these terms were not established with their present meanings until the Church made of them recognized and articulated Christian virtues. “3 Andere Wertsysteme und andere Denkmodelle brachten andere Urteilsdimensionen ins Spiel. So verdanken wir,

2 Galton, F.: „Measurement of Character“, in: Fortnightly Revue, 42 (1884), S. 181.

3 Allport, G. W./Odbert, H. S.: „Trait-Names: A Psycho-lexical Study“, in: Peterson, J. (Hrsg.): Psychological Monographs, Princeton 1938, S. 2. 
wie die Autoren ebenfalls klar erkannten, dem jahrhundertealten Glauben an die Astrologie die Entstehung von Eigenschaftsbegriffen wie „lunatic, jovial, saturine, and mercurial ", der irregeleiteten, aber gleichwohl über einen langen Zeitraum hinweg höchst populären Temperamentslehre des römischen Arztes Galenus, Charakterisierungen wie ,choleric, melancholic, phlegmatic, good-humored, bad-humored, as well as coldblodded, hearty, heartless, and cordial ". Die protestantische Reformation wiederum bereicherte das Spektrum der Urteilsgesichtspunkte um Begriffe wie „sincere, pious, bigoted, precise, selfish“, während das im 17. und 18. Jahrhundert dominierende aristokratische Denken Termini wie ,callous, countrified, disingenious, radical, prim, demure, gawky, enthousiastic, interesting and boresome" beisteuerte. Und auch die Psychologie selbst lieferte, wie Allport und Odbert vermerkten, ihren Beitrag zu diesem nach wie vor expandierenden Vokabularium, mit Begriffen wie ,introverted, extroverted, neurotic, regressive ... and the like.“4

\section{Nomen est omen?}

So leicht es nun allerdings der Sprachgemeinschaft fiel, ein linguistisches Instrumentarium zu schaffen, das ihren Mitgliedern die Möglichkeit bietet, sich in höchst nuancierter Form über die Wesensmerkmale zu verbreiten, die sie bei anderen Personen wahrzunehmen glauben, so schwer fiel es dieser Gemeinschaft, sich darauf zu einigen, woran man das Vorliegen einer bestimmten Eigenschaft erkennen könne. Obgleich zahllose Versuche unternommen wurden, in dieser Hinsicht zu einem Konsensus zu gelangen, gibt es bis heute keine Übereinkunft auf die man sich - im Sinne eines Kodes, der die semantische Beziehung zwischen Zeichen und Bezeichnetem verbindlich regelt - bei der Zuschreibung von Adjektiven berufen könnte, kein Lexikon, in dem man nachschlagen könnte, welche konkreten Verhaltensweisen das Vorliegen einer ganz bestimmten Eigenschaft anzeigen. Angefangen von der grauen Vorzeit bis in die jüngste Gegenwart hinein blieb es deshalb stets dem Belieben des Einzelnen anheim gestellt zu entscheiden, nach welchen Gesichtspunkten er sein Gegenüber beurteilt, welches Prozedere er seiner Urteilsbildung dabei zugrunde legt, und aufgrund welcher Kriterien er ihm bestimmte, in seiner Epoche als wichtig erachtete Eigenschaften, wie heutzutage etwa Glaubwürdigkeit, Zuverlässigkeit, Kompetenz usw. zuschreibt oder abspricht.

4 Allport/Odbert (wie Anm. 3), S. 2f. 
Dass dieser Beurteiler in der Regel nun aber wenig Mühe hat, sich sein Urteil zu bilden, ja, dass sich ihm oft sogar in Sekundenschnelle ein Eindruck von den Eigenschaften seines Gegenübers förmlich aufdrängt, ist oft als ein Beleg dafür angesehen worden, dass es sich bei diesen Begriffen keineswegs um bloße, in leere Worthülsen gepackte Fiktionen handeln könne, wie dies seit dem Altertum immer wieder behauptet worden war. Selbst die Tatsache, dass diese Termini oft noch lange Zeit im Gebrauch bleiben, nachdem die Gedankenwelt, die zu ihrer Entstehung Anlass gegeben hatte, längst als Trugschluss erkannt worden war, weckte kaum je Zweifel an der Annahme, dass Eigenschaftsbegriffe real existierende Sachverhalte bezeichnen. Denn nur die Konservierung von Termini, die sich auf tatsächlich vorhandene psychische Dispositionen beziehen, mache, wie speziell Allport \& Odbert betonten, für die Nutzer dieses Vokabulars Sinn, während diese sich nur selbst schaden würden „by preserving through names erroneous belief in merely ficticious or fabulous entities. ${ }^{5}$ Ungeachtet der über die Jahrhunderte hinweg erfolglos gebliebenen Bemühungen, verlässliche Indizien für das Vorliegen bestimmter Persönlichkeitseigenschaften zu finden, sei daher gleichwohl anzunehmen, dass sich die Persönlichkeitsforschung ,not with artifial facts but with genuine components of personality ${ }^{\text {“6 }}$ befasse. Denn: „A relationship that is difficult to trace is not necessarily a relationship that does not exist." 7

\section{Der heimliche Konsensus in der Bewertung von Eigenschaftsbegriffen}

Dass der Gebrauch von Eigenschaftsbegriffen, die sich auf rein fiktive Sachverhalte beziehen, dem Sprachnutzer nun aber keineswegs zum Nachteil geraten muss, sondern dass im Gegenteil, gerade der fehlende Konsensus bezüglich der empirischen Referenten von Persönlichkeitseigenschaften sich für die Nutzer dieses Vokabulars speziell im sozialen Kontext als höchst vorteilhaft erweisen kann, ist erst nach und nach entdeckt worden.

Der wohl wichtigste Anstoß dazu ging von den Arbeiten des amerikanischen Psychologen Norman H. Anderson aus. Angeregt durch einige bereits in den 50er Jahren des vergangenen Jahrhunderts durchge-

5 Allport/Odbert (wie Anm. 3), S. 2.

6 Allport/Odbert (wie Anm. 3), S. 12, im Original m. H.

7 Allport/Odbert (wie Anm. 3), S. 5. 
führte Studien ${ }^{8}$ versuchte Anderson Anfang der 1960er Jahre abzuklären, ob und in welchem Ausmaß Menschen mit unterschiedlichen Adjektiven unterschiedliche Wertvorstellungen verbinden. Zu diesem Zweck destillierte er aus dem riesigen, von Allport \& Odbert compilierten Eigenschaftsvokabular in einem mehrstufigen experimentellen Verfahren eine Liste von insgesamt 555 Adjektiven, die durchweg als im Alltag gebräuchlich und nützlich für die Charakterisierung von Personen angesehen werden konnten. In einem zweiten Arbeitsschritt ließ er jedes dieser Adjektive von jeweils 50 Studentinnen und Studenten der University of California, Los Angeles, im Hinblick auf die Frage beurteilen, ob es sich dabei um eine eher erwünschte oder um eine eher unerwünschte Persönlichkeitseigenschaft handle. Die Versuchspersonen wurden dabei instruiert, sich eine Person vorzustellen, die die jeweilige Eigenschaft besitze und gebeten, je nach dem Grade, in dem sie sich zu dieser Person hingezogen fühlten, das jeweilige Adjektiv auf einer 7-stufigen, von Null (äußerst unerwünscht) bis 6 (höchst erwünscht) reichenden Ratingskala einzustufen. ${ }^{9}$ In einem abschließenden dritten Arbeitsschritt wurden an drei anderen amerikanischen Universitäten (Ohio State University, University of Iowa, University of California, San Diego) Vergleichsuntersuchungen mit dem Ziel durchgeführt zu klären, ob und inwieweit sich die in Los Angeles erzielten Ergebnisse verallgemeinern ließen.

Die von Anderson im Jahre 1968 publizierten Mittelwerte und Streuungen für die 555, von jeweils 100 Probanden bewerteten Persönlichkeitseigenschaften gaben erstmals zu erkennen, dass die Mitglieder einer Sprachgemeinschaft mit dem ihnen zur Verfügung stehenden Eigenschaftsvokabular außerordentlich fein abgestufte und dabei höchst uniforme Wertvorstellungen verbinden. Gerade so als sei jeder Skalenpunkt auf der Erwünschtheitsskala mit einem ganz bestimmten Eigenschaftsbegriff besetzt, folgten die in Andersons Publikation gemäß ihres Erwünschtheitsgrades geordneten Mittelwerte einander dicht auf dicht: Bei einer Skalenspannweite, die sich mit einem Maximalwert von 5,73

8 Edwards, A. L.: „The relationship between the judged desirability of a trait and the probability that the trait will be endorsed“", in: Journal of Applied Psychology, 37 (1953), S. 90-93; Lövaas, O. I.: „Social desirability ratings of personality variables by Norwegian and American college students", in: Journal of Abnormal and Social Psychology, 57 (1958), S. 124-125; Rosen, E.: „Self appraisal, personal desirability and perceived social desirability of personality traits“", in: Journal of Abnormal and Social Psychology, 52 (1956), S. 151-158.

9 Anderson, N. H.: Likableness ratings of 555 personality-trait adjectives. Unpublished mimeo, University of California, Los Angeles 1964. 
(für das Adjektiv „sincere“) und einem Minimalwert von 0,26 (für „liar“) praktisch von einem Extrem der Skala zum andern erstreckte, betrug die Differenz zwischen benachbarten Eigenschaftsbegriffen im Durchschnitt lediglich winzige 0,01 Skalenpunkte bei einer gleichermaßen minimalen Streuung dieser Differenzwerte von $\mathrm{s}=0,01$. Höchst bemerkenswert war darüber hinaus der Befund, dass sich keinerlei geschlechtsspezifische Unterschiede in der Wertschätzung der verschiedenen Adjektive nachweisen ließen. Es zeigte sich vielmehr, dass über alle 555 Adjektive hinweg eine derart hohe numerische Übereinstimmung in den Werten der beiden Gruppen bestand, dass Anderson eine nach Geschlechtsgruppen getrennte Auflistung der Erwünschtheitswerte als unnötig erachtete und dementsprechend lediglich die für die Gesamtgruppe errechneten Mittelwerte und Streuungen publizierte.

Der in diesen Befunden erstmals zutage getretene, erstaunlich weit reichende Konsensus in der differenzierenden Wertschätzung von Eigenschaftsbegriffen wurde durch die Ergebnisse der Vergleichsuntersuchungen auf geradezu schockierende Weise bestätigt. Die Korrelationen zwischen den in Los Angeles erhobenen Daten und den an den drei anderen amerikanischen Universitäten erhobenen Datensätze lagen durchweg in einem Bereich zwischen .96 und .99 und erreichten damit eine Höhe, für die es aus der humanwissenschaftlichen Forschung praktisch kein Gegenstück gibt. Die Urteilskonkordanz, die sich in diesen beispiellos hohen Korrelationskoeffizienten dokumentiert, erwies sich zudem als derart robust, dass sie sich sowohl gegenüber Variationen im Umfang der Adjektivliste als auch in der Anzahl der Probanden als völlig unempfindlich erwies. So betrug die Korrelation zwischen den Mittelwerten der in Los Angeles erhobenen Daten und den Mittelwerten, die aus den Urteilen gewonnen wurden, die 160 Studenten der Ohio State University über 554 der 555 Adjektive aus Andersons Liste abgaben, .98. Bei einer mit 76 weiblichen und 53 männlichen Probanden der University of Iowa durchgeführten Untersuchung, die eine auf 140 Items verkürzte Adjektivliste beurteilten, betrug die Korrelation mit den entsprechenden Datensätzen aus Los Angeles .96 bzw. .97. Und selbst bei einer radikalen Beschränkung der Adjektivliste auf 20 zufällig aus Andersons Liste ausgewählte Begriffe, die einer Beurteilerstichprobe von nur 20 Probanden vorgelegt wurden, blieb, wie ein Vergleich der an der University of California San Diego mit den in Los Angeles erhobenen Daten zeigte, die hohe Übereinstimmung erhalten. Die Korrelation betrug in diesem Falle $.992 .{ }^{10}$

10 Anderson, N. H.: „Likableness ratings of 555 personality-trait words“, in: Journal of Personality and Social Psychology, 9 (1968), S. 278. 


\section{Die soziale Natur von Eigenschaftsbegriffen}

Die in Andersons Untersuchungsergebnissen zutage getretene, extrem hohe Übereinstimmung in der Bewertung von Eigenschaftsbegriffen gibt unschwer zu erkennen, dass das von der Sprachgemeinschaft geschaffene Eigenschaftsvokabular ganz und gar im Dienste der Regulation zwischenmenschlicher Beziehungen steht. Anders als dies Allport \& Odbert vermutet hatten und anders als dies uns allen auf den ersten Blick erscheinen mag, diente (und dient) das scheinbar zur Unterscheidung menschlicher Wesenszüge geschaffene linguistische Instrumentarium keineswegs der Optimierung einer wie auch immer gearteten ,Menschenkenntnis'. Es war (und ist) für diesen Zweck vielmehr allein schon deshalb untauglich, weil die Sprachgemeinschaft jedwedem für das Vorliegen einer bestimmten Persönlichkeitseigenschaft in Frage kommenden empirischen Indiz seit eh und je die Anerkennung verweigerte. Unter der Rubrik „Charakterkunde“ oder „Persönlichkeitspsychologie“ war das im Verlaufe vieler Jahrhunderte entstandene, gemäß der Wertvorstellungen der jeweiligen Epoche immer wieder ergänzte und so buchstäblich ins Unermessliche erweitere Adjektivrepertoire denn auch in einen Denkzusammenhang eingeordnet worden, der der tatsächlichen Funktion dieses Vokabulars wenig gerecht wird.

Spätestens der von Anderson aufgedeckte, verblüffend hohe Konsensus hinsichtlich der Wertvorstellungen, die die Mitglieder der Sprachgemeinschaft mit den verschiedenen Eigenschaftsbegriffen verbinden, hat jedoch die zutiefst soziale Natur dieser Termini unübersehbar deutlich gemacht. Denn es versteht sich von selbst, dass in dem Maße, in dem die Nutzer dieses Vokabulars hinsichtlich der Erwünschtheit der zur Charakterisierung von Personen verwendeten Begriffe übereinstimmen, die Zuschreibung dieser Termini bedeutsame Konsequenzen für das ,Ansehen' einer Person und damit auch für deren Akzeptanz im sozialen Umfeld nach sich ziehen muss. Je höher diese Übereinstimmung ist, desto mehr eröffnet sich für den Attribuierenden die Möglichkeit, die Wertschätzung, die einer Person von Dritten entgegengebracht wird, durch die Auswahl entsprechender Adjektive in sowohl positiver als auch negativer Hinsicht zu beeinflussen. Da nun aber, wie aus den von Anderson berichteten Korrelationen hervorgeht, ein solch weitgehender Konsensus ganz offenkundig für ein riesiges Spektrum an Eigenschaftsbegriffen existiert, eignet sich dieses Vokabular fraglos hervorragend als ,Kode ' für die stillschweigende Verständigung zwischen den Mitgliedern der Sprachgemeinschaft über den Wert oder Unwert einer Person. 
Das Dunkel, das die Frage der empirischen Referenten von Persönlichkeitseigenschaften umgibt, erweist sich dabei für den Attribuierenden keineswegs als ein Handicap, sondern geradezu als Freibrief für die wahlfreie Nutzung dieses Vokabulars. Denn der Spielraum für die Möglichkeit, durch die Zuschreibung von Adjektiven den sozialen ,Kurswert“ einer Person steigen oder auch fallen $\mathrm{zu}$ lassen, ist selbstverständlich umso größer, je weniger der Attribuierende befürchten muss, für die $\mathrm{Zu}$ schreibung von Eigenschaftsbegriffen zur Rechenschaft gezogen zu werden. Der seit alters her fehlende Konsensus hinsichtlich der Indizien, die für die Eigenschaftsattribution herangezogen werden, bietet den Mitgliedern der Sprachgemeinschaft somit nicht nur die fraglos verlockende Möglichkeit, sich bei der Zuschreibung von Adjektiven von der Beweislast weitestgehend zu befreien. Die ad infinitum verlängerte Suche nach sog. ,verlässlichen' Kriterien der Persönlichkeitsbeschreibung diente (und dient) ihnen vielmehr darüber hinaus auch als eine bequeme Legitimation für die Perpetuierung der seit Urzeiten geübten Praxis, selbstherrlich zu entscheiden, aufgrund welcher Anzeichen man anderen Personen bestimmte Eigenschaften zuschreibt oder abspricht.

\section{Die zeitliche Stabilität der mit Eigenschaftsbegriffen verbundenen Wertvorstellungen}

Der ,wahre Charakter' des riesigen, von Allport \& Odbert aufgezeigten Adjektivrepertoires, entpuppt sich somit vor dem Hintergrund des sorgsam gepflegten Dissens hinsichtlich der empirischen Referenten von Persönlichkeitseigenschaften und dem weithin existierenden Konsens hinsichtlich deren Erwünschtheit denn auch als der eines heimlichen Referenzsystems zur Bewertung des ,sozialen Gebrauchswerts' von Personen. Mit andern Worten: Das Adjektivvokabular erfüllt im Rahmen der zwischenmenschlichen Verständigung gewissermaßen die Funktion einer ,Währung', in der das Individuum an der ,sozialen Börse' gehandelt wird.

Die zentrale Voraussetzung für die Nutzbarkeit dieser Währung ist nun allerdings - wie bei jeder anderen Währung - deren Stabilität. Denn in dem Maße, in dem sich - beispielsweise im Zuge eines sich wandelnden Zeitgeistes - der Konsens hinsichtlich der Wertschätzung der sprachlich zur Verfügung stehenden Adjektive verflüchtigt, umso geringer ist auch der, die betreffende Person sozial auf- oder abwertende Effekt zu veranschlagen, der durch die Attribution bestimmter Eigenschaften erzielt werden kann. 
Zur Prüfung der Frage, ob und inwieweit sich der von Anderson aufgezeigte, extrem hohe Konsens auch über einen längeren Zeitraum hinweg als stabil erweist, habe ich gemeinsam mit dem amerikanischen Politologen Roger Masters in den Jahren 1995 und 1996 am Dartmouth College Wiederholungsuntersuchungen mit der von Anderson erstellten Adjektivliste durchgeführt. Die Ergebnisse dieser Untersuchungen erbrachten den höchst bemerkenswerten Befund, dass - ungeachtet der seit den 1960er Jahren eingetretenen, oft als ,Zerfall“ zuvor gültiger Wertsysteme apostrophierten gesellschaftlichen Veränderungen - die Studentengeneration der 1990er Jahre kaum weniger dezidierte Wertvorstellungen mit den in Andersons Liste aufgeführten 555 Adjektiven verband als dies 30 Jahre zuvor der Fall war.

Mit einem Maximalwert von 5,58 und einem Minimalwert von 0,31 war die Spannweite der Mittelwerte der Erwünschtheit sowohl auf dem positiven als auch auf dem negativen Ast der Erwünschtheitsskala nur geringfügig niedriger als dies bei den von Anderson erhobenen Daten der Fall war. Mit einer mittleren Differenz von nur 0,01 Skalenpunkten zwischen den gemäß ihres Erwünschtheitsgrades geordneten Adjektiven und einer gleichermaßen minimalen Streuung dieser Differenzwerte von $\mathrm{s}=0,01$ waren darüber hinaus auch die Urteile, die die Studenten des Dartmouth College über die in Andersons Liste enthaltenen Adjektive abgaben, genau so fein abgestuft wie diejenigen, die Anderson seinerzeit von den Studenten der UCLA erhalten hatte.

Selbst im Hinblick auf die Bewertung der Erwünschtheit jedes einzelnen dieser Adjektive zeigte sich erneut und auf höchst eindrucksvolle Weise der von Anderson aufgedeckte, extrem weitreichende Konsensus. Wie aus dem im Anhang (S. 132-138) dargestellten Vergleich der in den Jahren 1995 und 1996 von jeweils 34 bzw. 39 Studenten des Dartmouth College gewonnenen Mittelwerten der Erwünschtheit unmittelbar ersichtlich wird, ergab sich für praktisch jede der 555 in Andersons Liste enthaltenen Persönlichkeitseigenschaften eine nahezu identische Bewertung. Die sich in diesen beiden Verlaufskurven dokumentierende, extrem hohe Korrelation von .99 bestätigte einmal mehr die Existenz der von Anderson ermittelten, bis dahin kaum für möglich gehaltenen Übereinstimmung der Mitglieder der Sprachgemeinschaft hinsichtlich des Erwünschtheitsgrades der verschiedenen, im Adjektivrepertoire enthaltenen Eigenschaftsbegriffe.

Einen nicht weniger bemerkenswerten Befund lieferte zudem der Vergleich der von den Studenten des Dartmouth College erhobenen Erwünschtheitswerte mit den entsprechenden Werten, die Anderson mehr 
als 30 Jahre zuvor in Los Angeles - praktisch von der Elterngeneration dieser Studenten - erhalten hatte. Ungeachtet der seit Anfang der 1960er Jahre eingetretenen weitreichenden politischen, ökonomischen und ideologischen Veränderungen in der Gesellschaft korrelierten sowohl die 1995 als auch die 1996 erhobenen durchschnittlichen Erwünschtheitswerte noch immer in einer Höhe von .95 mit den entsprechenden von Anderson publizierten Mittelwerten.

\section{Sozialer Druck}

In ihrer Tragweite für das theoretische Verständnis sowohl der Beziehungsregulation zwischen den Mitgliedern der Sprachgemeinschaft als auch hinsichtlich der mit diesem Prozess eng verknüpften kulturellen Entwicklung der Gesellschaft sind Befunde dieser Art wohl kaum zu überschätzen. Denn das Vorhandensein eines weithin gebräuchlichen, über einen bemerkenswert langen Zeitraum hinweg fast unverändert gültig bleibenden Referenzsystems zur Bewertung von Persönlichkeitseigenschaften hat nicht nur Auswirkungen im Hinblick auf die Wertschätzung, die den einzelnen Mitgliedern dieser Gemeinschaft in ihrem sozialen Umfeld zuteil wird. Die bloße Existenz eines derartigen Referenzsystems muss sich vielmehr auf Seiten der Individuen in Form eines erheblichen „sozialen Drucks“ bemerkbar machen, der auf deren Handeln unmittelbar Einfluss nimmt. Und zwar indem er darauf hinwirkt, den Einzelnen zu veranlassen, sich in einer Art und Weise zu verhalten, die vorteilhafte und nicht etwa nachteilige Auswirkungen im Hinblick auf die im sozialen Umfeld ausgelöste Eigenschaftsattribution nach sich zieht.

Denn in einer Welt, in der praktisch sämtliche materiellen Ressourcen, die der Einzelne zum Überleben benötigt, sozial kontrolliert werden, kann es diesem nicht gleichgültig sein, was andere, von ihm halten'. Da der Zugang zu diesen Ressourcen - angefangen von der Aufnahme in eine bestimmte Peer-Gruppe, über die Höhe des einer Person gewährten Einkommens, bis hin zu politischen Wahlentscheidungen - letztlich von dem Bild abhängt, das sich andere von ihr machen, muss dieser Person zwangsläufig daran gelegen sein zu erreichen, dass ihr bestimmte Eigenschaften zugeschrieben, bestimmte andere nicht zugeschrieben werden. Die Beantwortung der so lange rätselhaft gebliebenen Frage, nach welchen Kriterien der Beurteiler zu seinem ,Bild vom Andern' gelangt, ist für das Verständnis der conditio humana denn auch von grundlegender Bedeutung. 


\section{Mehr als tausend Worte}

Man würde denken, dass homo loquens, der sprechende Mensch, allein schon wegen der Begrenztheit seines Blickfelds für seine Meinungsbildung hauptsächlich Informationen heranzieht, die ihm auf verbalem Wege von anderen Personen zugänglich gemacht wurden. Und tatsächlich hätte der Mensch ohne den Zugang zu der in den Köpfen seiner Mitmenschen vorhandenen Information, den ihm die Erfindung der Sprache überhaupt erst eröffnet hat, einen sehr engen geistigen Horizont. Er wüsste rein nichts von der Existenz der zahllosen Dinge, die sich außerhalb jenes winzigen Flecks der Erdoberfläche vorfinden, auf dem er sich bewegt. Er hätte zudem keinerlei Ahnung von all dem, was sich an diesem Ort abspielte bevor er geboren war. Und er könnte, was für seine geistige Entwicklung wohl am schlimmsten wäre, nicht von den Ideen und dem Wissen seiner Mitmenschen profitieren. Ja, er wüsste nicht mal, ob und in welcher Weise die Vorstellungen und Meinungen im Kopfe seines Gegenübers sich von denjenigen in seinem eigenen unterscheiden. Für die ganz und gar auf die Erweiterung unserer Erkenntnisse ausgerichtete Wissenschaft galt denn auch schon immer der logos, das Wort, als das kommunikative Medium par excellence, neben dem die Bedeutung jedes anderen Informationsmediums zu verblassen scheint.

Dass wir alle, in krassem Gegensatz hierzu, dazu tendieren, uns ganz und gar auf die visuelle Eindrucksbildung zu verlassen, ist, wenn man dem Volksmund glauben darf, ein offenes Geheimnis. „Ein Bild sagt mehr als tausend Worte", so verherrlichend äußern sich Sprichwörter aller Völker und Kulturen über das, was der Mensch „mit eigenen Augen" in Erfahrung bringt. Und so geringschätzig, ja deklassierend kommentieren sie das, was aus dem Munde seines Gegenübers kommt. Dass es sich bei diesem Diktum nicht um leere Worte handelt, sondern dass da vielmehr einer tiefen Überzeugung Ausdruck verliehen wird, zeigt sich allein schon an der ungeheuren Faszination, die die visuellen Medien seit eh und je auf die Menschheit ausübten - angefangen von der camera obscura über die Daguerrotypie, den Film bis hin zu unseren modernen TV-Sets, denen nachgesagt wird, dass sie die Aufmerksamkeit dermaßen fesseln, dass sie die sprachliche Kommunikation in der Familie nahezu zum Erliegen bringen.

Belege zuhauf finden sich in der menschlichen Kulturgeschichte auch dafür, dass die dem Auge zugeführte Information als besonders glaubwürdig eingestuft wird. „Seeing is believing“, so fasst ein englisches Sprichwort das tiefe Vertrauen in Worte, das der Mensch in 
seinen optischen Eindruck setzt. Die japanische Sprache beehrt gar den simplen Photoapparat mit dem Namen Shashinki, was wörtlich übersetzt „Maschine zur Abbildung der Wahrheit" bedeutet. In Analogie dazu gilt in unserem westlichen Sprachraum der ,Augenzeuge" als ein ganz besonders verlässlicher Informant. Selbst Kunstwerke, sei es nun in Form von Gemälden oder Statuen, erachten wir seit alters her als Quelle einer besonders tiefen Wahrheit. Dies, obwohl wir sehr wohl wissen, dass deren Anblick lediglich zu einem Eindruck verleitet, der vom Betrachter zwar als ,überwältigend' erlebt werden mag, dessen Wahrheitsgehalt dadurch aber keineswegs erwiesen ist.

\section{Ein kurzer Blick genügt}

Was aber tut das Bild zur Sache? Welchen Einfluss nimmt es auf die Meinungsbildung? Wenn man diese Frage klären will, ist es hilfreich, sich zunächst einmal zu überlegen, wie zu einer Zeit, als homo sapiens die Sprache noch nicht erfunden hatte, der Prozess der Meinungsbildung vonstatten ging. Die Antwort darauf ist einfach: Er verließ sich auf seine fünf Sinne! Das heißt, er machte es wie die Tiere, die sich ja auch eine Meinung bilden müssen über die in ihrer Umwelt vorhandenen Gegebenheiten, wenn sie darin zurechtkommen wollen. Und sie bilden sich diese Meinung bekanntlich meist schon in Sekundenschnelle, ohne dass ihnen dies viel Kopfzerbrechen bereiten würde - es genügt ihnen ein kurzer Blick.

Mit dem wahrhaft weltbewegenden Akt der Sprachentstehung, schuf sich der Mensch eine zweite, bis dahin gänzlich verschlossen gebliebene Informationsquelle. Diese eröffnet den Mitgliedern der Sprachgemeinschaft erstmalig die Möglichkeit, sich eine Meinung zu bilden, die nicht nur auf den vom Sinnesapparat angelieferten Informationen beruht. Mit Hilfe der durch den verbalen Diskurs erstmals zugänglich gemachten Information kann die aufgrund sensorischer Impressionen entstandene Meinung vielmehr um wesentliche Gesichtspunkte erweitert - oder gar völlig umgestoßen werden. Denn anders als die Tiere, deren Spontaneindruck für sie sozusagen das letzte Wort zur Sache darstellt und damit auch deren Handeln bestimmt, muss der Mensch dem Eindruck, den ihm seine Sinne ungefragt aufdrängen, nicht unbedingt Glauben schenken. Durch die mit der Entwicklung des Neokortex neu entstandene Möglichkeit zu kritischer Reflexion, ist er vielmehr erstmals in der Lage, zwischen dem Für und Wider des eigenen Eindrucks und der ihm verbal zur Kenntnis gebrachten Sichtweise anderer Personen sorgfältig abzuwägen 
- und auf diese Weise die von seinem Sinnesapparat schnellschussartig angelieferten Vor-Urteile durch Nach-Denken zu überwinden.

Ob und inwieweit ihm dies tatsächlich gelingt, hängt nun allerdings davon ab, ob die von unserer Spezies meist als recht mühsam empfundenen Aktivitäten des Zuhörens, des Lesens und des Nachdenkens überhaupt zustande kommen. Dass dies erfolgt ist keineswegs zwingend. Denn der über Jahrmillionen hinweg evolutionär entstandene, quasi zu unserer ,biologischen Grundausstattung' gehörende Mechanismus der sensorischen Eindrucksbildung wurde durch die Erfindung der Sprache ja nicht außer Kraft gesetzt. Er arbeitet vielmehr nach wie vor, „unbeirrbar und unbelehrbar", wie es der Verhaltensforscher und Nobelpreisträger Konrad Lorenz einmal ausdrückte, gemäß seiner eigenen, archaischen Gesetze. Insofern besteht für das Individuum natürlich immer die Option, auf das was ihm andere sagen wenig oder nichts zu geben, es als ,schwer verständlich ' zurückzuweisen oder es gar als ,falsch` oder ,irrelevant ${ }^{\star}$ abzutun - und stattdessen sozusagen auf ,Autopilot ${ }^{`} \mathrm{zu}$ schalten und sich einfach von den reflexartig entstehenden unbewussten Schlüssen leiten zu lassen, die ihm sein sensorischer Apparat anliefert, sozusagen ,gratis frei Haus', ohne jede kognitive Anstrengung. Und so stellt sich denn auch die Frage, ob durch die Verschiebung der Balance vom Akustischen ins Optische, die für unsere Gegenwart so charakteristisch ist, homo loquens, der sprechende Mensch, sich künftig immer mehr auf seinen sensorischen Spontaneindruck verlassen wird - und damit in seinem ganzen Denken und Handeln auf einen Modus der Informationsverarbeitung zurückfällt, den man eigentlich längst überwunden glaubte.

\section{„The great communicator"}

Dass die Wissenschaft schon seit ihren frühen Anfängen ganz und gar auf den logos setzte, auf den bloßen Augenschein dagegen nie viel gab, hat gute Gründe: Wenn die Dinge so wären, wie sie uns erscheinen, brauchte es keine Wissenschaft. Und doch zeigt sich heute, dass der weitgehende Verzicht auf die Untersuchung der Frage, welchen Einfluss die visuelle Eindrucksbildung auf unsere Meinungsbildung und auf unser Gefühlsleben ausübt, schwerwiegende, wenn nicht gar verhängnisvolle Konsequenzen zeitigte. Denn er bewirkte, dass die Wissenschaft mit leeren Händen da stand, als sie Ende der 40er Jahre des vergangenen Jahrhunderts mit der Frage konfrontiert wurde, welche Wirkungen sich aus der visuellen Zeitenwende ergeben würden, die mit der Einführung des Fernsehens gerade begonnen hatte. 
Ein halbes Jahrhundert nachdem das neue visuelle Medium seinen kometenhaften Aufstieg zum dominierenden Medium der Massenkommunikation begann, zeigt sich jedoch, in zunehmend klareren Umrissen, mit welcher Macht der visuelle Eindruck den menschlichen Verstand beherrscht. Im Krieg der Bilder gegen die Urteilskraft der Bürger werde der menschliche Verstand der große Verlierer sein, so deutete schon Ende der 1980er Jahre der amerikanische Publizist Hedrick Smith die Zeichen der Zeit: „Das Auge siegt über das Ohr, Bild schlägt Ton.“11

Anlass für diese Prophezeiung war ein Mitte der 80er Jahren in der politischen Szene ganz neu aufgetauchtes Phänomen: Die von vielen Beobachtern des politischen Geschehens mit Verblüffung registrierte Reaktion der Öffentlichkeit auf die Auftritte des amerikanischen Präsidenten Ronald Reagan. Worte, so schien es, konnten dem als ,the great communicator" apostrophierten ehemaligen Hollywood-Schauspieler schlechterdings nichts anhaben. Und zwar weder seine eigenen, die ihn als einen Präsidenten von ziemlich bescheidenem politischem Sachverstand erscheinen ließen, noch diejenigen, die in der Presse über ihn veröffentlicht wurden - und die diesen Eindruck nachdrücklich zu bestätigen suchten. Selbst Skandale seiner Administration, die in zahllosen Negativschlagzeilen von den Zeitungen publik gemacht wurden, blieben aus unerfindlichen Gründen nicht an ihm haften.

In dem Versuch, das rätselhafte, noch nie dagewesene Phänomen begrifflich $\mathrm{zu}$ fassen, prägte die amerikanische Presse schließlich den Ausdruck „Teflon-Effekt“. Er bezeichnet das merkwürdige Phänomen, das bestimmte politische Akteure beim Wahlvolk auf Sympathie und Nachsicht für politische Positionen und Handlungen stoßen, die es anderen Politikern, denen „das gewisse Etwas“ abgehe, höchst übel nehmen würde.

Wo dieses gewisse Etwas zu suchen sei, das Reagan praktisch immun gegen die von der Presse vorgetragene Kritik machte, dafür hatte schon Reagans Medienberater Michael Deaver einen Tipp gegeben: „Das Bild“, so erläuterte Deaver Reagans Wirkung auf das Wahlvolk, ,erzählt die ganze Geschichte, egal was Ronald Reagan sagt.“" ${ }^{12}$ Konsequenterweise pflegten sich die Mitarbeiter Ronald Reagans, wie der deutsche Politologe Thomas Meyer berichtet, bei den Fernsehjournalisten denn ,auch für Verrißsendungen $\mathrm{zu}$ bedanken, wenn nur die Bilder

11 Smith, H.: Der Machtkampf in Amerika. Reagans Erbe: Washingtons neue Elite, Hamburg 1988, S. 450.

12 Smith (wie Anm. 11), S. 461. 
stimmten.“13 Und tatsächlich lassen die inzwischen vorliegenden Untersuchungsergebnisse zur Medienwirkung politischer Funktionsträger kaum noch daran zweifeln, dass das Wohl und Wehe des modernen Politikers in erster Linie davon abhängt, ob wir, die Fernsehzuschauer, finden, dass ,die Bilder stimmen“.

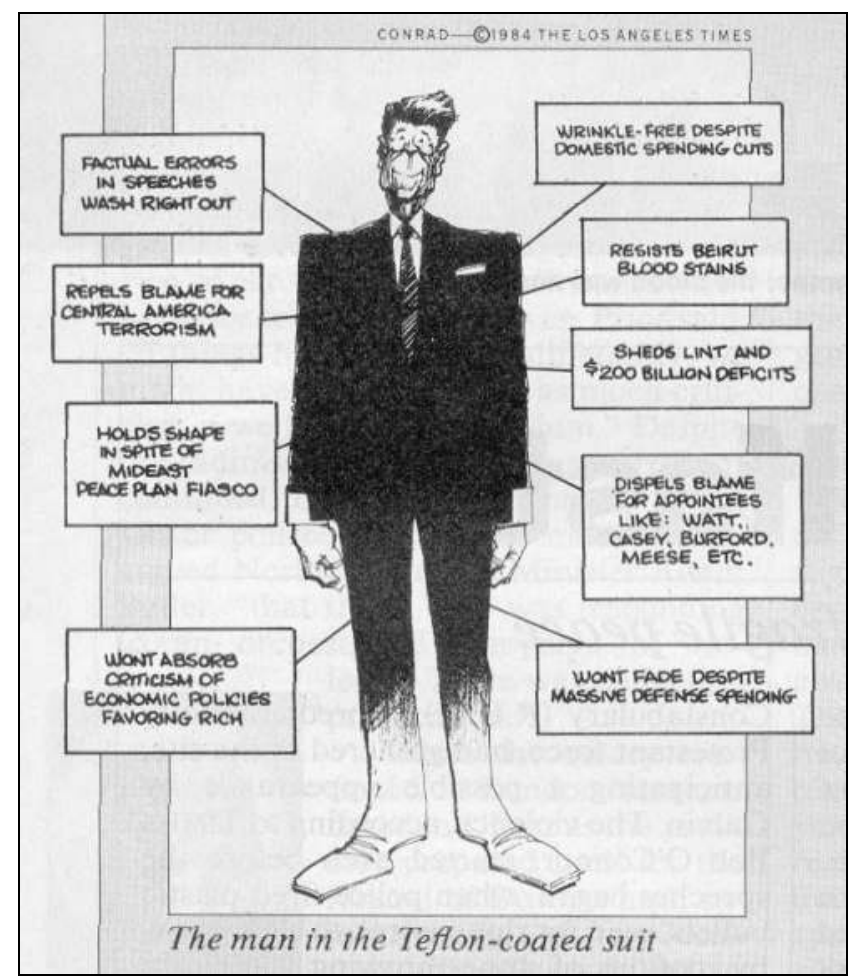

\section{Die Macht des Bildes}

Wie nachhaltig das auf dem Bildschirm dargebotene nonverbale, gestische und mimische, Verhalten das Bild prägt, das sich die Regierten von den Regierenden machen, ist erstmals Mitte der 1990er Jahre in vollem Umfang deutlich geworden. Zusammen mit Roger Masters und Alfred Raveau, meinen Teamkollegen in der am Maison des Sciences de l'Hom$m e$ in Paris wirkenden internationalen Arbeitsgruppe „Visual Communications Research Group", hatten wir damals beschlossen, in

13 Meyer, T.: Die Inszenierung des Scheins, Frankfurt 1992, S. 48. 
Deutschland, Frankreich und den USA eine Untersuchung durchzuführen, die klären sollte, ob das Image von Politikern etwas mit den images zu tun haben könnte, die allabendlich, in Form ganz kurzer, in die TV-Nachrichten eingeblendeter Bewegtbilder über die Bildschirme der Nation huschen.

Die Analyse der kognitiven und emotionalen Reaktionen von mehr als 200 amerikanischen, deutschen und französischen Probanden auf tonlos dargebotene Videoclips von insgesamt 180 politischen Akteuren machte deutlich, dass wir beim Auftritt eines Politikers auf dem Bildschirm buchstäblich in Sekundenschnelle entscheiden, was wir von ihm halten. Dabei ist es für die Geschwindigkeit der Urteilsbildung völlig unerheblich, ob wir die abgebildete Person bereits kennen und somit eine vorgefasste Meinung nur aus dem Gedächtnis abzurufen brauchen oder ob wir sie nicht kennen und diese Meinung uns erst bilden müssen. Die Zeitspanne, die der optische Apparat benötigt, um ein Urteil zu fällen, in dem sich beispielsweise entscheidet, ob wir eine auf dem Bildschirm auftauchende Person sympathisch finden, als langweilig erachten, als arrogant, unehrlich, intelligent, hinterhältig, fair u.a.m. einstufen, beträgt gerade mal 250 Millisekunden - eine Viertelsekunde. ${ }^{14}$

\section{Bilder Gourmet}

$\mathrm{Ob}$ dieses ganz mühelos, ohne jede kognitive Anstrengung entstandene Urteil für die betroffene Person schmeichelhaft oder aber vernichtend ausfällt, hängt, nach allem, was wir bisher wissen, von Reizgegebenheiten ab, die oft geradezu erschütternd einfach strukturiert sind. ${ }^{15}$ Und doch üben gerade diese einen tief greifenden Einfluss nicht nur auf das Bild aus, das wir uns vom Andern machen, sondern auch auf unsere innere, emotionale Einstellung zu dieser Person. Denn bezüglich der Reize, die auf nonverbalem Wege angeliefert werden, erweist sich der Mensch, wie die Untersuchungen der letzten Jahre vielfach gezeigt haben, keineswegs als ,Allesfresser', sondern geradezu als ,Gourmet': Es gibt nonverbale Stimuli, an denen sich der Betrachter buchstäblich nicht

14 Frey, S.: „Neue Wege in der Kommunikationsforschung“, in: Ganten, D./ Meyer-Galow, E./Ropers, H.-H./Scheich, H./Schwarz, H./Urban, K./ Truscheit, E. (Hrsg.): Gene, Neurone, Qubits \& Co. Unsere Welten der Information, Stuttgart 1999, S. 56f.

15 Frey, S.: Die Macht des Bildes. Der Einfluss der nonverbalen Kommunikation auf Kultur und Politik, Bern 1999, S. 135ff. 
satt sehen kann, und es gibt andere, die er sozusagen aus tiefstem Herzen verabscheut - meist einschließlich der Person, die sie serviert.

Obwohl es sich bei derartigen Gefühlsregungen um zutiefst innerpsychische Vorgänge handelt, sind diese doch keine reine Privatangelegenheit, sondern haben gewichtige soziale Konsequenzen. Indem nämlich die nonverbalen Verhaltensweisen, die uns von einer Person vor Augen geführt werden, unsere innere, emotionale Einstellung zu ihr prägen, kommt diesen Phänomenen eine zentrale Bedeutung auch im Hinblick auf die Chancen und Entwicklungsmöglichkeiten zu, die dieser Person von ihrem sozialen Umfeld einräumt werden. Denn in dem Maße, in dem wir uns auf unseren optischen Eindruck verlassen, hängt es letztlich vom Ergebnis eines ganz automatisch, in Form unbewusster Schlüsse ablaufenden Urteilsprozesses ab, welche Eigenschaften wir einer Person zuschreiben oder absprechen. In Anbetracht des extrem hohen Konsensus in der Bewertung dieser Eigenschaften, wie er zuerst in Andersons Ergebnissen und später auch in unseren eigenen Untersuchungen zutage getreten ist, bedeutet dies nicht weniger, als dass ein gänzlich unreflektiertes Entscheidungsprozedere darüber befindet, welche Person mit der Übernahme welcher Aufgaben und Funktionen betraut wird.

\section{Rückkehr der Natur}

Man braucht nicht viel Phantasie, um sich die Folgen einer Entwicklung auszumalen, die dazu führt, dass die Autorität der Bilder den Prozess der Meinungsbildung immer mehr dominiert. Die Konsequenzen davon würden sich wahrscheinlich zuerst in einer zunehmenden Verflachung im Bereich der Geisteswissenschaften bemerkbar machen. Sie würden jedoch wohl auch schon mittelfristig die ganze kulturelle Entwicklung und vor allem die Innovationskraft der Menschen empfindlich schwächen, die ja in erster Linie von deren Fähigkeit abhängt, umdenken zu können. Möglicherweise hat in dieser Hinsicht die Zukunft ja überhaupt schon begonnen.

Im Bereich des politischen Lebens hätte eine sich immer stärker durchsetzende Tendenz, das, was wir mit den Augen wahrnehmen, buchstäblich so zu nehmen als sei es wahr, zudem die unweigerliche - auch für die Politiker selbst demütigende, wenn nicht gar demoralisierende Konsequenz, dass die Karrierechancen der Kandidaten für öffentliche Ämter immer weniger von deren politischem Sachverstand abhängen und immer mehr von deren Fähigkeit, den im wahrsten Sinne des Wortes 
,oberflächlichen' Kriterien zu genügen, aufgrund derer die visuelle Eindrucksbildung zu ihrem Urteil gelangt.

Auch in dieser Hinsicht hat, falls Hedrick Smith und Thomas Meyer Recht haben sollten, die historische Wende bereits stattgefunden. Denn aus der Sicht dieser Autoren markiert der Regierungsantritt des gelernten Schauspielers Ronald Reagan den Beginn einer Epoche, in der die politischen Entscheidungen von den Fakten völlig abgekoppelt sind, wobei an die Stelle eines politischen Programms die Inszenierung des Erscheinungsbildes des Kandidaten tritt. „An was wollen Sie glauben, an Fakten oder an Ihre Augen?“, so lüfteten, wie Smith ${ }^{16}$ berichtet, Reagans Medienberater lachend das Geheimnis, wie sie praktisch mühelos die Zustimmung der amerikanischen Öffentlichkeit zu Ronald Reagans vielfach höchst kontroversen politischen Entscheidungen gewannen. Im dem tiefen, kaum je enttäuschten Vertrauen darauf, dass für den vor dem Bildschirm thronenden Souverän letztlich eben nicht die Fakten, sondern die Faxen zählen, probte dieser Präsident Pressekonferenzen denn auch wie selbstverständlich tagelang, und zwar, wie $\operatorname{Meyer}^{17}$ zu berichten weiß, „bis in die Gesten und scheinbaren Zufallsbemerkungen am Rande hinein“. Die enorme Publikumswirkung, die der amerikanische Präsident mit diesem Verfahren in den USA erzielt habe, werde, wie Meyer schon Anfang der 1990er Jahre vorhersagte, wohl überall dort, wo in technischer Hinsicht „die Voraussetzungen verfügbar sind, allen seine Gesetze aufzwingen, die den Erfolg nicht fahrlässig verspielen wollen. “18

Die weitreichenden gesellschaftlichen Konsequenzen, zu denen diese Art der kollektiven Urteilsbildung im Bereich des politischen Lebens führen muss, hat der große amerikanische Karikaturist Herbert Block in seiner unvergleichlichen Art bereits in den 1980er Jahren offen gelegt, als er in einer Serie berühmter, den viel zitierten Tefloneffekt Reagans illustrierender Cartoons dem amerikanischen Souverän den Spiegel vor die Nase hielt. Dass dieser sich darin erkannte, und sein Entscheidungsprozedere überdacht oder gar revidiert hätte, kann freilich angesichts des in der Nachfolge Reagans von ihm angestellten, von Hedrick Smith als „Washingtons neue Elite“ bezeichneten, Personals mit Fug und Recht bezweifelt werden. Und selbst die Vorhersage Meyers ist, wie ein Blick auf die Runde der politischen Funktionsträger zeigt, denen der europäische - und nicht zuletzt der deutsche - Wähler das Steuer für das Rad der Geschichte in die Hand gab, schon sehr rasch eingetroffen. Denn

16 Smith (wie Anm. 11), S. 450.

17 Meyer (wie Anm. 13), S. 94.

18 Meyer (wie Anm. 13), S. 94. 
dieser Blick gibt unschwer zu erkennen, dass selbst in unseren Breiten, in denen traditionsgemäß eine im Vergleich zu Amerika weitaus rauere Art der nonverbalen Stimulation verabreicht wurde, die Aspiranten für politische Ämter inzwischen klar erkannt haben, nach welchen Kriterien der Souverän seine Personalentscheidungen trifft - und sich umgehend darauf eingestellt haben.

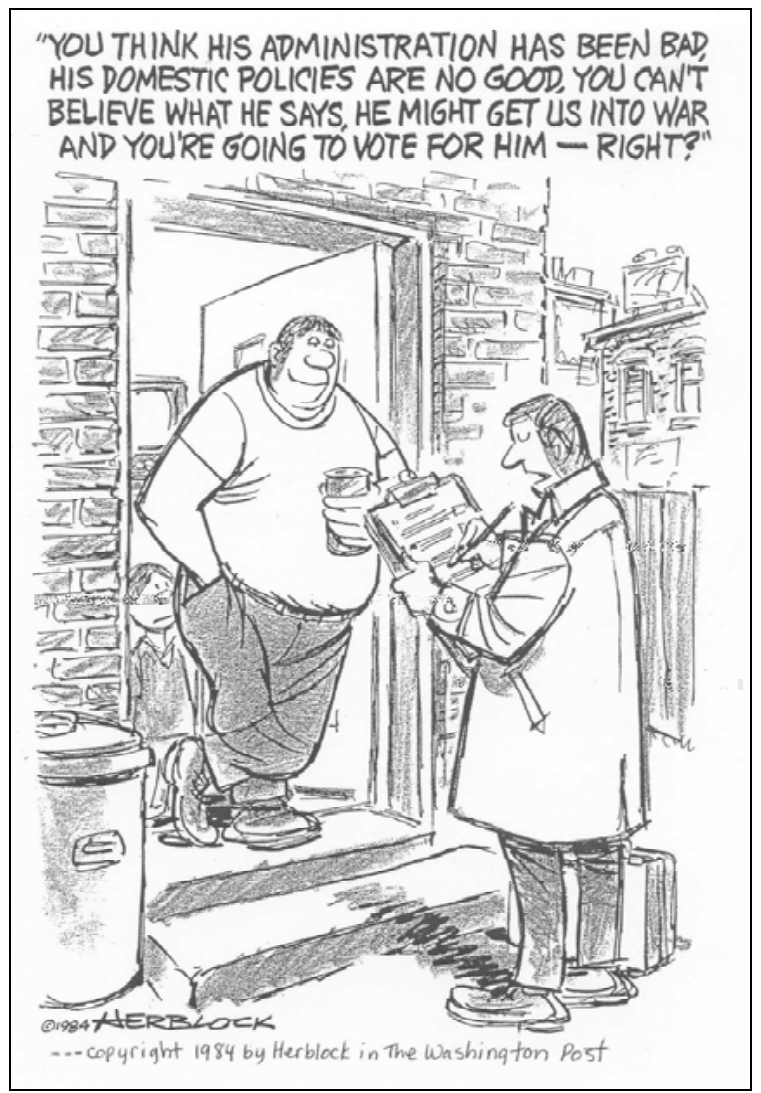

Zumindest für den Bereich der Politik ist, wie zu befürchten steht, denn auch bereits abzusehen, wohin die zunehmende Verschiebung der Balance vom Akustischen ins Optische führen wird: Politiker, die nicht willens oder nicht fähig sind, dem sie beäugenden Souverän das ihm mundende nonverbale Futter zu reichen, werden verschwinden. An ihrer Stelle wird ein neuer, weitaus geschmeidigerer Typus von Politiker auf der Bildfläche erscheinen, der gewissermaßen maßgerecht für das Auge des Betrachters gezüchtet wird. 
Aber nicht nur in der Welt da draußen, auch in unserer Innenwelt wird die durch den Siegeszug der Bildmedien eingeleitete visuelle Zeitenwende die Weichen neu stellen. Denn in dem Maße, in dem der visuelle Sinn die Macht über den Prozess der Meinungsbildung zurückgewinnt, die er schon immer nur höchst widerwillig mit dem Neokortex teilte, wird in unserem so reich ausgestatteten, durch die Einflüsse einer Jahrtausende alten Kulturgeschichte geprägten Kopf eine Entwicklung einsetzen, wie man sie aus der Geschichte alter Hochkulturen kennt: Die Natur holt sich das an die Kultur verlorene Terrain zurück, und der Urwald überwuchert wieder die Stätten einstiger Blüte. 
Anhang:

SOCIAL APPRECIATION OF 555 PERSONALITY TRAITS

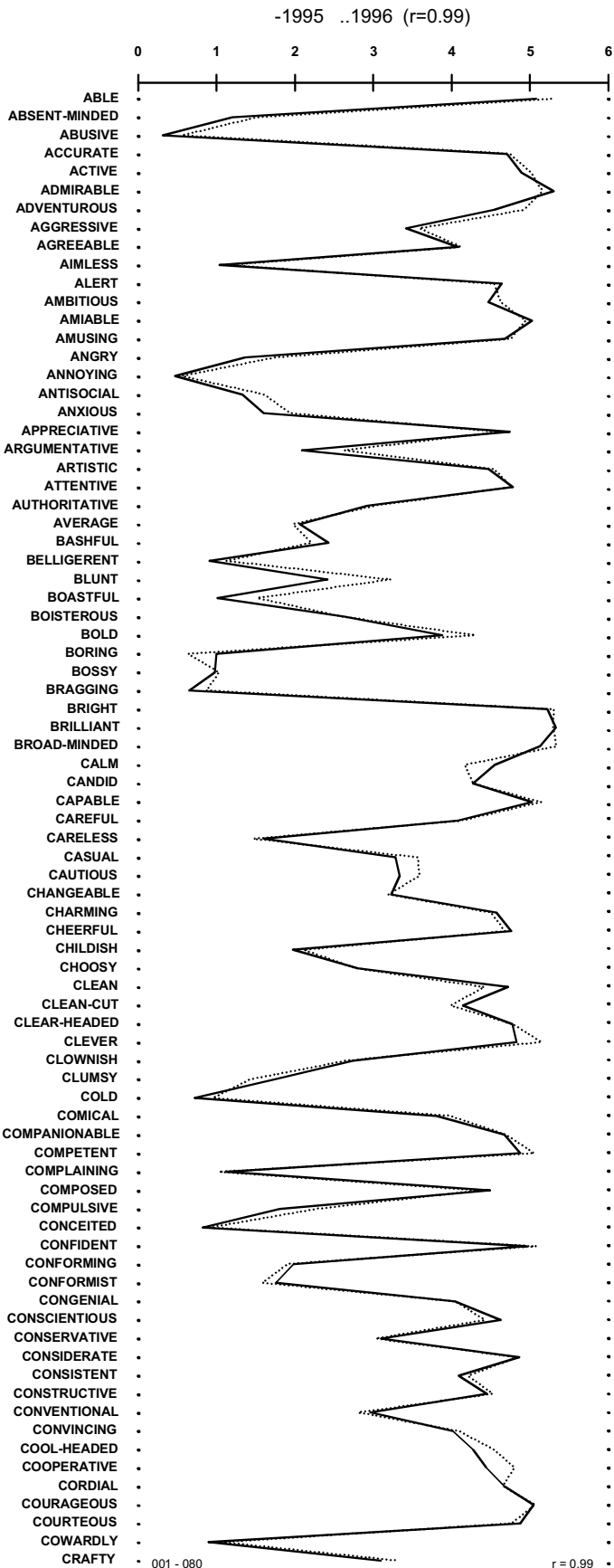


SOCIAL APPRECIATION OF 555 PERSONALITY TRAITS

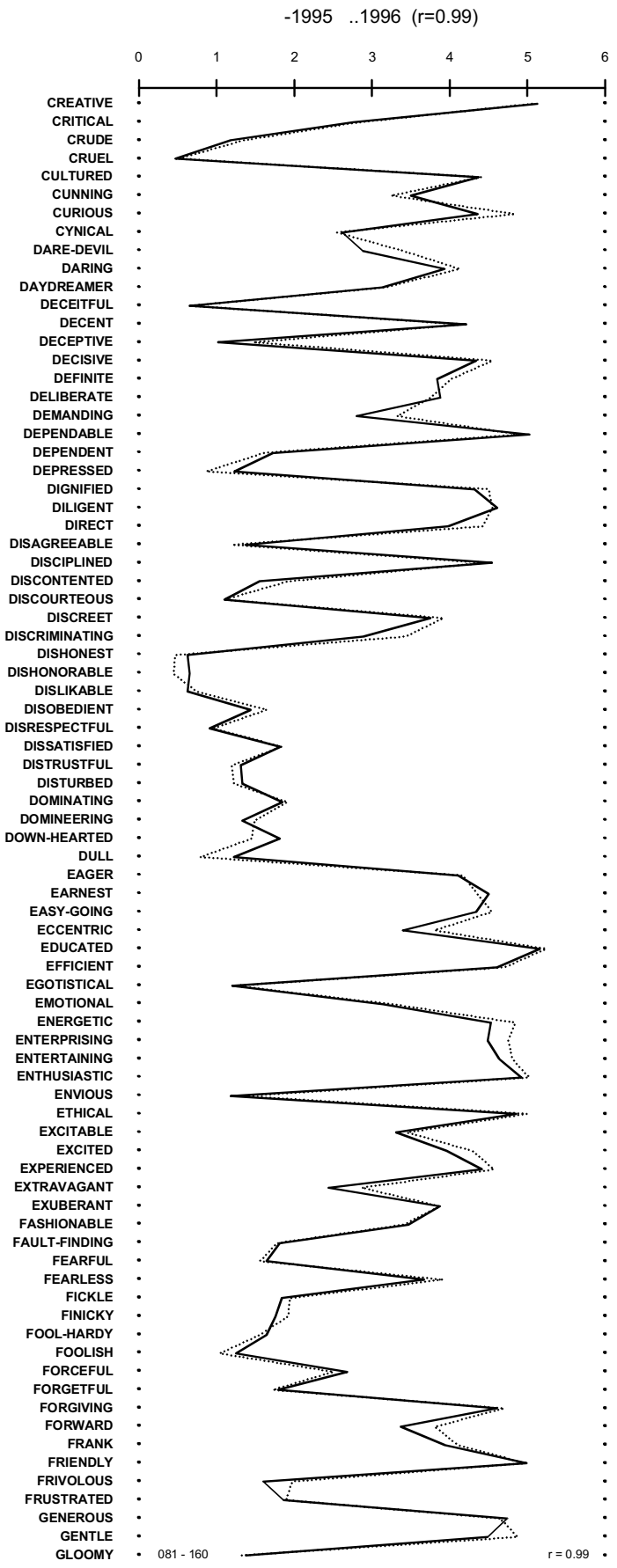


SOCIAL APPRECIATION OF 555 PERSONALITY TRAITS

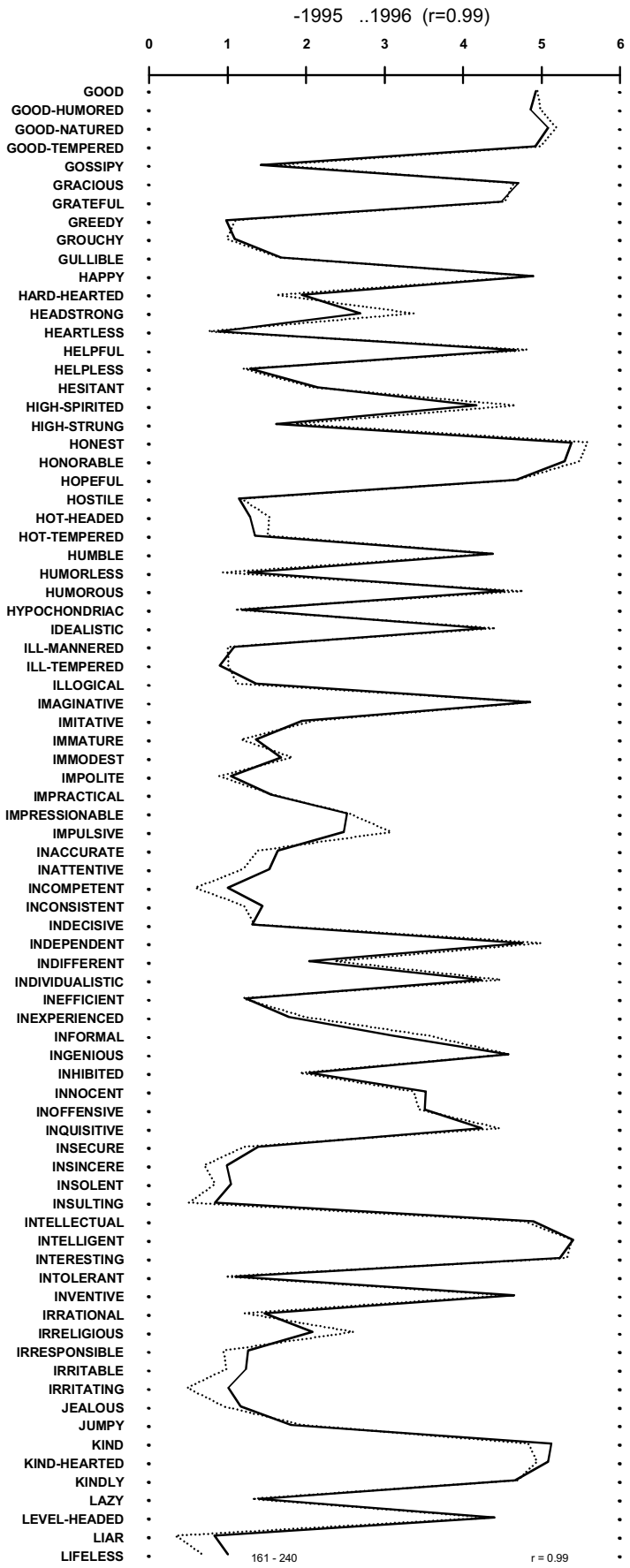


SOCIAL APPRECIATION OF 555 PERSONALITY TRAITS

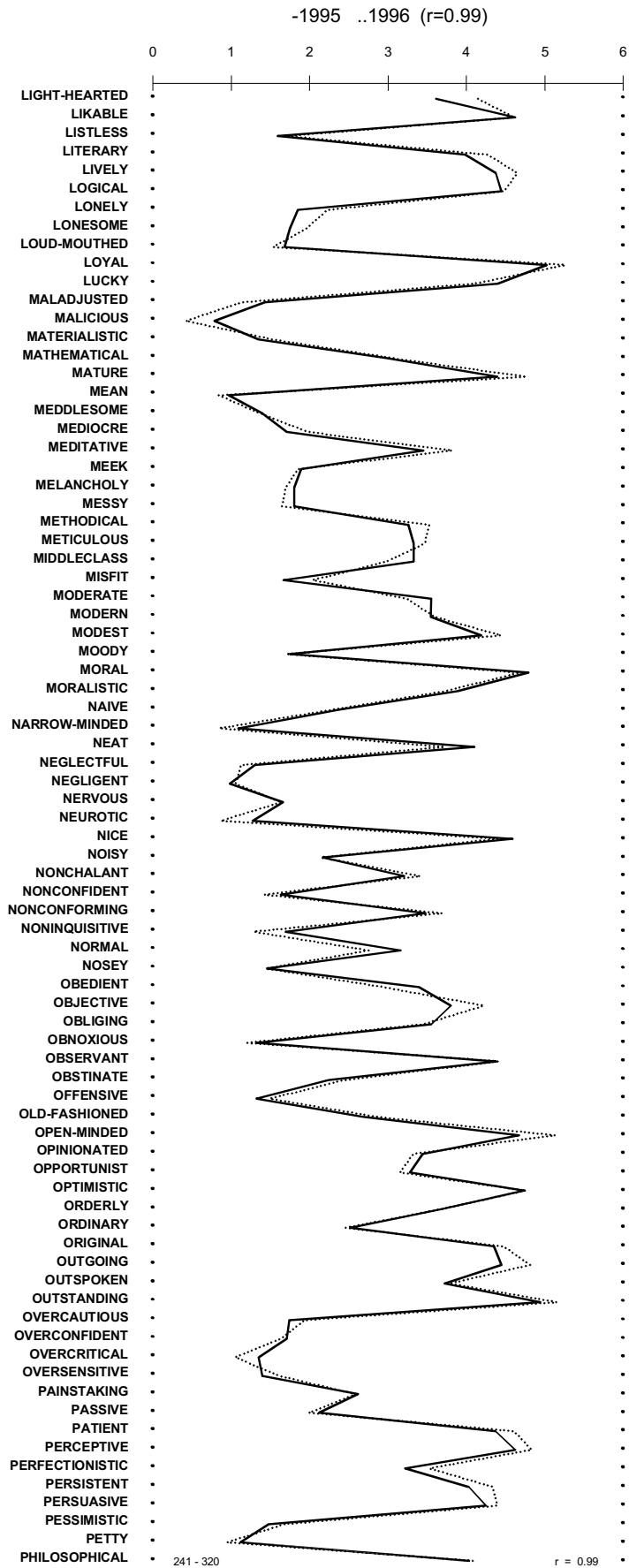


SOCIAL APPRECIATION OF 555 PERSONALITY TRAITS

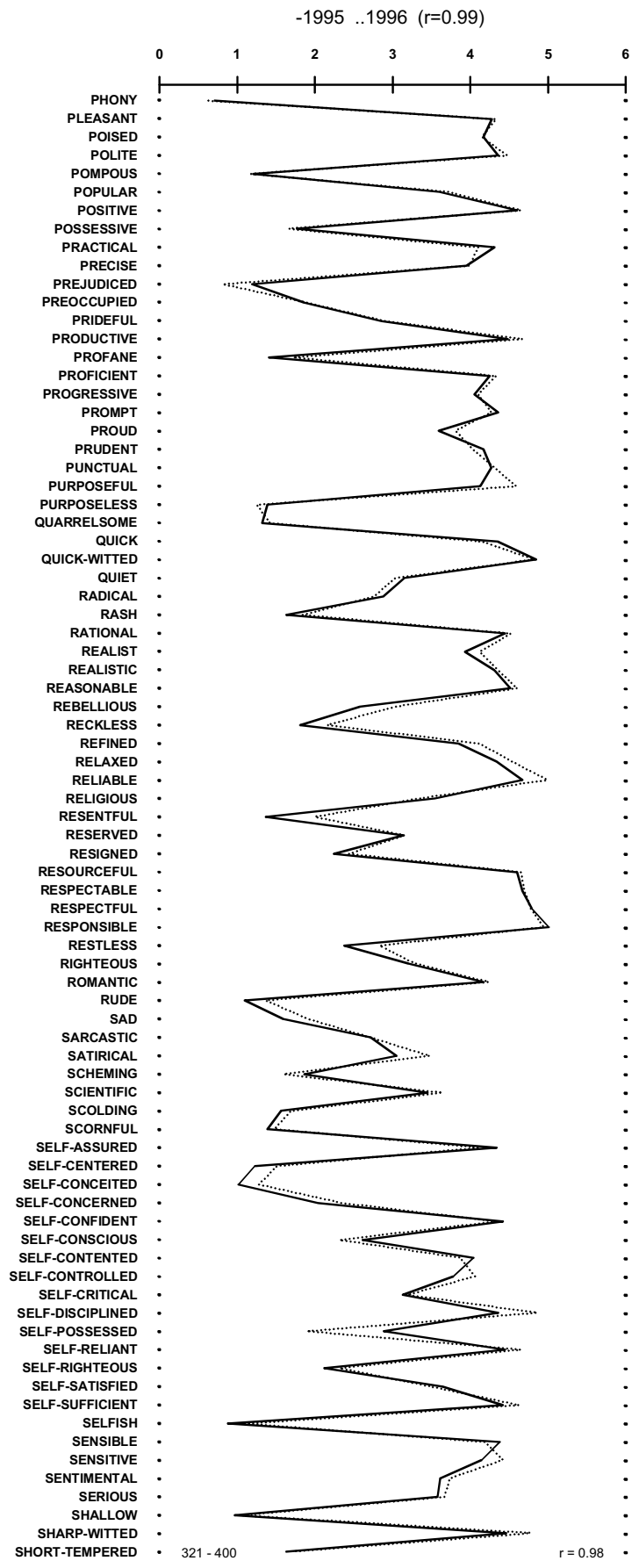


SOCIAL APPRECIATION OF 555 PERSONALITY TRAITS

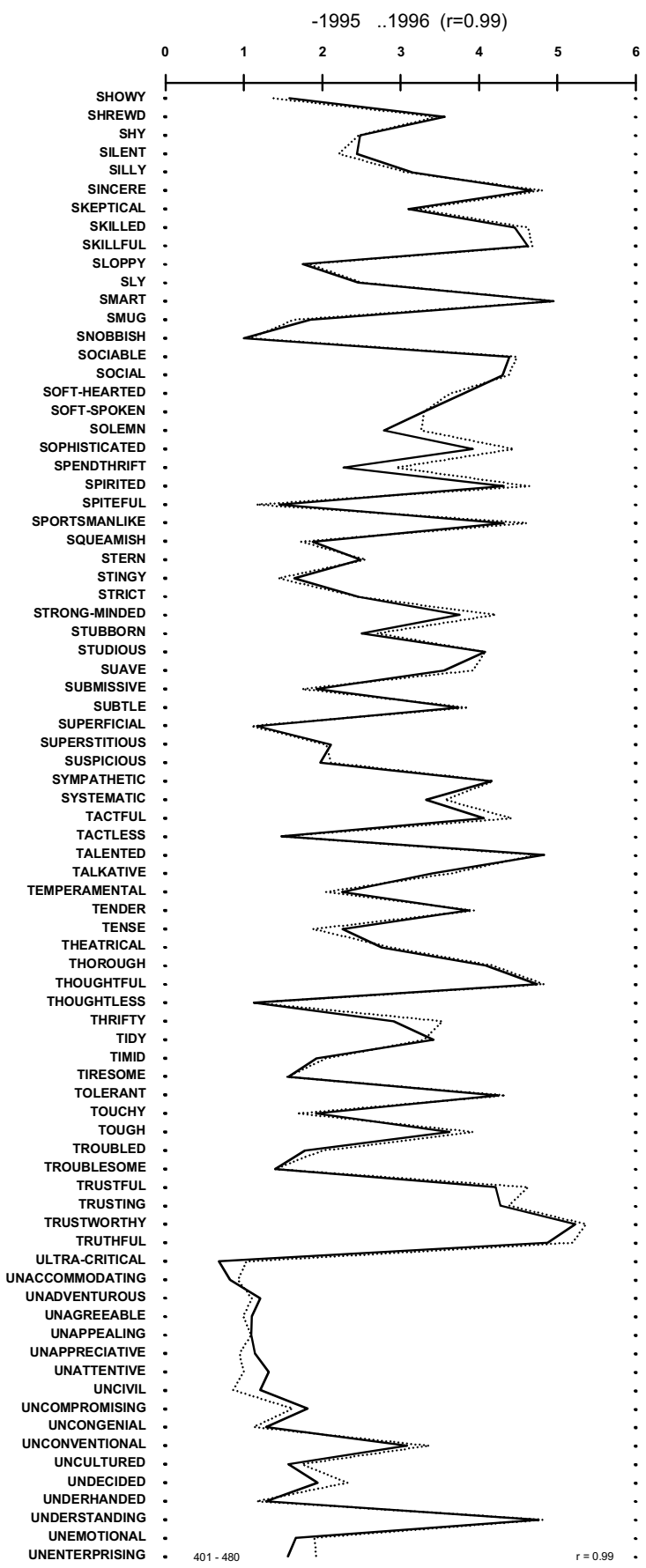


SOCIAL APPRECIATION OF 555 PERSONALITY TRAITS

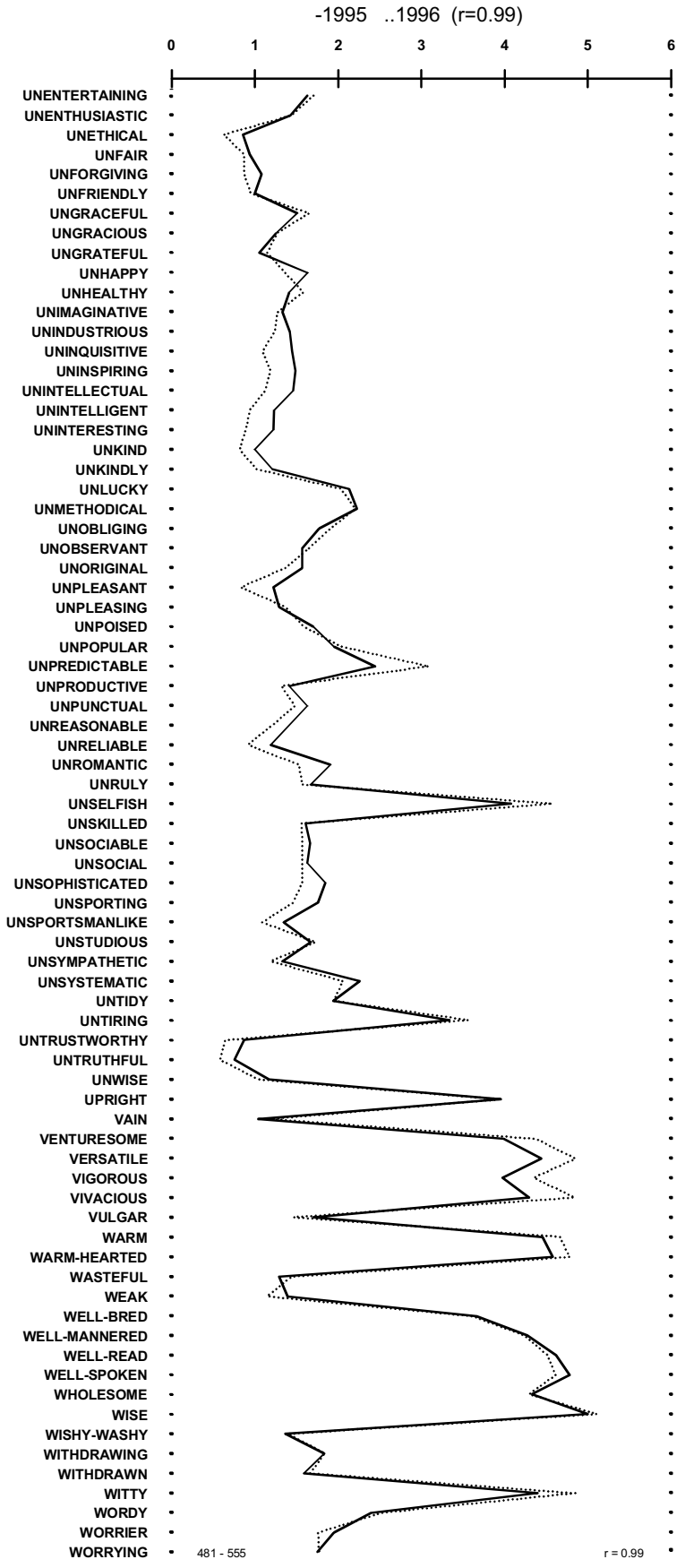


ECKART ALTENMÜLLER

\title{
MUSIK - DIE SPRACHE DER GEFÜHLE?
}

\author{
NEUROBIOLOGISCHE GRUNDLAGEN \\ EMOTIONALER MUSIKWAHRNEHMUNG
}

\section{Musik als soziales Phänomen}

Es ist ein Allgemeinplatz, dass Musik besonders starke Emotionen auslösen kann. Musizieren und Musikhören wird von der Mehrzahl der Deutschen immer noch als die wichtigste Freizeitbeschäftigung angesehen. Etwa 4 Millionen Mitbürger musizieren regelmäßig an einem Instrument oder singen in einem Chor. ${ }^{1}$ Die neurobiologischen und anthropologischen Grundlagen dieser Vorliebe für Musik sind bislang wenig erforscht. Es ist weitgehend ungeklärt, wie Musik ihre Wirkungen auf die Emotionssysteme entfaltet. Offen ist auch, ob musikalische Ausdrucksmittel universell, Kultur übergreifend, ähnliche Reaktionen bei Hörern auslösen. Grundsätzlich stellt sich schließlich die Frage, warum Musik als zweites Kommunikationssystem neben der Sprache bis in die Neuzeit erhalten blieb.

Bevor wir uns der Frage nach der emotionalen Wirkung von Musik zuwenden, beginnen wir mit dem Versuch einer Definition von Musik. Im weitesten Sinn kann Musik als nach Regeln gestaltetes, in der Zeit strukturiertes akustisches Muster verstanden werden. In dieser reduktionistischen Musikdefinition fehlt allerdings noch eine wesentliche Eigenschaft von Musik, nämlich ihr kommunikativer Aspekt. Man sollte also eher sagen, Musik ist die bewusst gestaltete, zeitlich strukturierte Ordnung von akustischen Ereignissen in sozialen Kontexten. Als Argument für diese erweiterte Definition kann angeführt werden, dass Musik in zahlreichen sozialen Kontexten stattfindet und häufig spezifische Funktionen erfüllt. Ein viel zitiertes Beispiel sind die Wiegenlieder, die der Mutter-Kind-Bindung und wahrscheinlich auch dem Spracherwerb die-

1 Gembris, Heiner: Grundlagen musikalischer Entwicklung, Augsburg 1998. 
nen. ${ }^{2}$ Als weiteres Beispiel wird oft die Rolle von Musik bei der Werbung um Sexualpartner herangezogen. ${ }^{3}$ Hier handelt es sich möglicherweise um die Demonstration verborgener Qualitäten. ${ }^{4}$ Man kann sich gut vorstellen, dass das Singen eines jungen Mannes nicht nur ästhetischen Zwecken dient, sondern auch Auskunft über seine Gesundheit geben kann. Denn ein kräftiger Sänger leidet höchst wahrscheinlich nicht unter einer floriden Lungentuberkulose, eine Information, die immerhin bis zu Beginn des letzten Jahrhunderts für eine potentielle Eheschließung von großer Bedeutung war. Die starke emotionale Wirkung, die von kräftigen Männerstimmen ausgeht - man denke an das berühmt-berüchtigte „hohe C“ der Tenöre -, könnte also mit einer derartigen Demonstration von Fitness in Zusammenhang gebracht werden. Aber es sind nicht nur die verborgenen Qualitäten des Musikanten, sondern auch direkte akustische Merkmale von Musik, die bestimmte Wirkungen entfalten. So wissen wir heute, dass ausdrucksvolles Musizieren zur Ausschüttung von Endorphinen führen kann, wodurch Glücksgefühle ausgelöst werden, die beim gemeinschaftlichen Hören der Intensivierung einer Bindung dienen können. ${ }^{5}$

Auf der Gruppenebene kommt Musik eine wichtige Rolle im $\mathrm{Zu}$ sammenhang mit Tanz zu. Tanz wird in zahlreichen Gesellschaften bei religiösen Festen und gesellschaftlichen Riten eingesetzt. Tanz fördert soziale Bindung und scheint über eine verstärkte Oxytoxin-Ausschüttung der Hypophyse eine stabilere Gedächtnisbildung zu bewirken. ${ }^{6}$ Damit wird die Erinnerung an ein spezifisches Gruppenerlebnis gefördert. In ähnlicher Weise wird Musik als Markersignal von Gruppenidentität bei zahlreichen anderen Gelegenheiten eingesetzt. Man denke nur an Nationalhymnen, Fußballgesänge und an die Identität stiftende Wirkung, die bestimmte Lieder von ethnischen Minderheiten in einem Staatswesen

2 Trehub, Sandra: „Musical predispositions in infancy: an update“, in: Peretz, Isabelle/Zatorre, Robert: The cognitive Neuroscience of Music, Oxford 2003, S. 57-78.

3 Miller, Geoffrey: „Evolution of human music through sexual selection“, in: Wallin, Nils/Merker, Björn/Brown, Steven (Hrsg.): The origins of music, Cambridge 2000, S. 315-328.

4 Vgl. Voland, Eckhart: „Das ,Handicap-Prinzip“ und die biologische Evolution der ästhetischen Urteilskraft", in diesem Band, S. 35-60.

5 Panksepp, Jaak/Bernatzki, Günter: „Emotional sounds and the brain: the neuro-affective foundations of musical appreciation“, in: Behavioural processes, 60 (2002), S. 133-155.

6 Huron, David: „Is music an evolutionary adaptation“, in: Peretz, Isabelle/ Zatorre, Robert (Hrsg): The cognitive Neuroscience of Music, Oxford 2003, S. 57-78. 
haben. ${ }^{7}$ Ein eindrucksvoller Hinweis auf die Wertschätzung, die Musik als Mittel zur Organisation sozialer Gruppen geniesst, ist der Einsatz von Musik beim Militär. Möglicherweise ist hier der vorrangige Zweck des Musizierens die Verhaltenssynchronisation. Dies kann auch beim Einsatz von Musik in der Arbeitsorganisation, etwa als „Spinnerlied“, „Dreschegesang" etc. angenommen werden. Naturgemäß sind derartige Funktionen von Musik heute mit der Differenzierung und Individualisierung von Arbeitsvorgängen in den Hintergrund gedrängt worden. Als weitere Eigenschaft sozialer „Wirkung“ von Musik kann ihr Einsatz als Heilmittel angesehen werden. Musizieren kann zu einer verbesserten Körperabwehr führen und kann Angst lösend wirken. In vielen Kulturen wird Musik als begleitende Therapie bei medizinischen Eingriffen durchaus sinnvoll eingesetzt. ${ }^{8}$

Zusammenfassend spricht der vielfältige Einsatz von Musik in sozialen Interaktionen für ihre wichtige Funktion bei der Organisation und Motivation von Gruppen. Nur durch eine starke Wirkung auf die Emotionssysteme können diese Funktionen erreicht und erhalten werden.

\section{Was sind Emotionen?}

Eine Emotion ist eine Reaktion auf einen bestimmten Reiz auf der Grundlage der Reizbewertung. Diese zunächst sehr allgemein gehaltene Definition ist mit den Sichtweisen von Zoologen, Neurobiologen und Psychologen vereinbar. Die Reaktion hängt von der Motivation ab, die wiederum an Hormone und Aktivität bestimmter neuronaler Netzwerke gebunden ist. Darüber hinaus hängen Emotionen von der Erfahrung ab, das heißt, sie sind Lernvorgängen unterworfen. Auch die Art der Reizbewertung kann entweder angeboren sein oder durch Lernen modifiziert werden. Im Zusammenhang mit der Emotionsdefinition bezeichnet der Begriff Motivation die Wahrscheinlichkeit, mit der ein bestimmter Reiz aufgesucht bzw. gemieden wird. Mit einem derartigen Wahrscheinlichkeitsmaß können in der Tierphysiologie Rückschlüsse auf mögliche von Tieren „erlebte“ Emotionen gezogen werden. Beim Menschen ist ein objektives Maß der Motivation weniger wichtig, da hier die Möglichkeit besteht, durch Introspektion Berichte über Gefühle zu erhalten.

Die oben aufgeführten trockenen Definitionen sollen an einem Beispiel erläutert werden: Man stelle sich den Besuch der Aufführung von

7 Kopiez, Reinhard/Brink, Gerhard: Fußball-Fangesänge. Eine Fanomenologie, Würzburg 1998.

8 Panksepp (wie Anm.5). 
Johann Sebastian Bachs' Matthäuspassion in einer altehrwürdigen Kathedrale am Karfreitagabend vor. In einer anrührenden Szene kurz nach dem Verhör durch die Hohenpriester wird Jesus von Pilatus der Menge vorgeführt und Pilatus fragt ,Welchen wollet ihr, dass ich Euch losgebe? Barrabam, oder Jesum, von dem gesagt wird, er sei Christus“. Daraufhin schreit die von den Hohenpriestern angestachelte Menge „Barrabam“. Musikalisch ist der Barrabas-Ruf vom Komponisten durch einen plötzlichen, sehr lauten und harmonisch unerwarteten dissonanten Akkord des Chores und Orchesters realisiert worden. Auch bei einem modernen Hörer führt dieser unerwartete Klang häufig zu einer starken emotionalen Reaktion, die sich unter anderem in einem den Rücken hinunter laufenden „Schauer“, in einem „Gänsehautgefühl“ manifestieren kann.

Die oben genannten Begriffe sollen nun auf dieses Phänomen angewandt werden. Der Reiz ist das von Chor und Orchester produzierte akustische Signal. Die Reaktion ist die unwillkürliche Aufrichtung der Körperbehaarung, ein vom autonomen Nervensystem ausgelöster Reflex, der uns als Gänsehaut bewusst wird. Die Reizbewertung ist im genannten Beispiel nicht ganz eindeutig. Eine angeborene Form der Reizbewertung wäre das Erschrecken durch die plötzliche Zunahme der Lautstärke. Die Reizbewertung wäre dann etwa so zu umschreiben: ,Vorsicht, es tritt etwas Neues auf, ich muss aufpassen, dass mir nichts passiert". Diese Reizantwort wird in der neurobiologischen Sprache als „Orientierungsreaktion" bezeichnet und erfolgt sehr rasch und unbewusst. Eine erlernte Form der Reizbewertung wären Gefühle des Mitleids beim Gedanken, dass hier ungerechterweise ein unschuldiger Mensch und Wohltäter vom Mob zum Tod verurteilt wird. Die Motivation wird bei den meisten $\mathrm{Zu}$ hörern hoch sein, da sie in der Regel wach und aufmerksam sind und ja auch freiwillig die Aufführung aufgesucht haben. Allerdings kann die Motivation durch eine besonders langweilige, qualitativ schlechte Aufführung sinken und dann wäre es mehr als fraglich, ob bei einem vor sich hin dösenden oder verärgerten Zuhörer überhaupt ein Gänsehauteffekt eintritt. Bei einem erfahrenen Hörer, der das Werk sehr gut kennt, kann sich umgekehrt schon in der Erwartung des starken musikalischen Moments eine Gänsehaut einstellen, ja vielleicht kann schon die reine Imagination dieser Stelle dazu führen. Wir hätten es dann mit einem konditionierten Reiz-Reaktionsschema zu tun.

Der Versuch einer Übertragung der Emotionsdefinition auf dieses musikalische Beispiel macht auch die Schwierigkeiten beim Beschreiben von Emotionen deutlich. Subjektive Empfindungen werden nämlich nicht berücksichtigt, obwohl sie ein ganz wesentliches Element in unse- 
rem Emotionsverständnis ausmachen. Die Definition der Emotion muss also wie folgt erweitert werden:

Beim Menschen versteht man unter einer Emotion ein Reaktionsmuster, das auf vier Ebenen wirksam wird: a) als subjektives Gefühl, b) als motorische Äußerung, z.B. als Ausdrucksverhalten in Mimik, Gestik, und Stimme, c) als physiologische Reaktion des autonomen Nervensystems z.B. in einer Gänsehaut und d) als kognitive Bewertung.

\section{Neurobiologie der Emotion}

Es existieren zahlreiche Emotionstheorien, die im Wesentlichen drei Urspungstheorien zugeordnet werden können: 1. die James-Lange Theorie, 2. die Cannon-Bard Theorie und 3. die Lazarus-Schachter Theorie. ${ }^{9}$

Die James-Lange-Theorie besagt vereinfacht, dass man fühlt, nachdem der Körper reagiert hat. „Ich bin fröhlich, weil ich lache“. Ein auslösender Reiz erzeugt auf subkortikaler Ebene ohne zwangsläufige Einschaltung des Bewusstseins eine Erregung des autonomen Nervensystems und eine motorische Aktion. Erst die bewusst wahrgenommene Erregung und die kognitive Interpretation der Handlung führen anschließend zur subjektiven Erfahrung einer Emotion.

Die zweite Theorie von Cannon und Bard dagegen postuliert, dass der auslösende Reiz zwei gleichzeitig ablaufende Reaktionen hervorbringt, nämlich die physiologische Erregung und die bewusste Wahrnehmung der Emotion. Keine der beiden Reaktionen bedingt die andere. Die Theorie geht also davon aus, dass die körperlichen Prozesse von den psychologischen unabhängig sind.

In einer dritten Theorie versuchten Lazarus und Schachter beide oben genannten Vorstellungen stärker in den Kontext einzubinden, in dem eine Emotion ausgelöst wird. Der bewusst wahrgenommene emotionale Reiz und ein momentanes physiologisches Erregungsniveau bestimmen gemeinsam, wie die Bewertung von Erregung und Reiz erfolgen wird. Sie lösen dann an Hand von Situationshinweisen und Kontexten die Reaktion aus und führen gleichzeitig zur Emotionserfahrung.

Die Mechanismen der Verarbeitung von Emotionen und die beteiligten Hirnstrukturen sind in Abb. 1 dargestellt.

9 Zimbardo, Rudolf/Gerig, Klaus: Allgemeine Psychologie, Heidelberg 1999. 


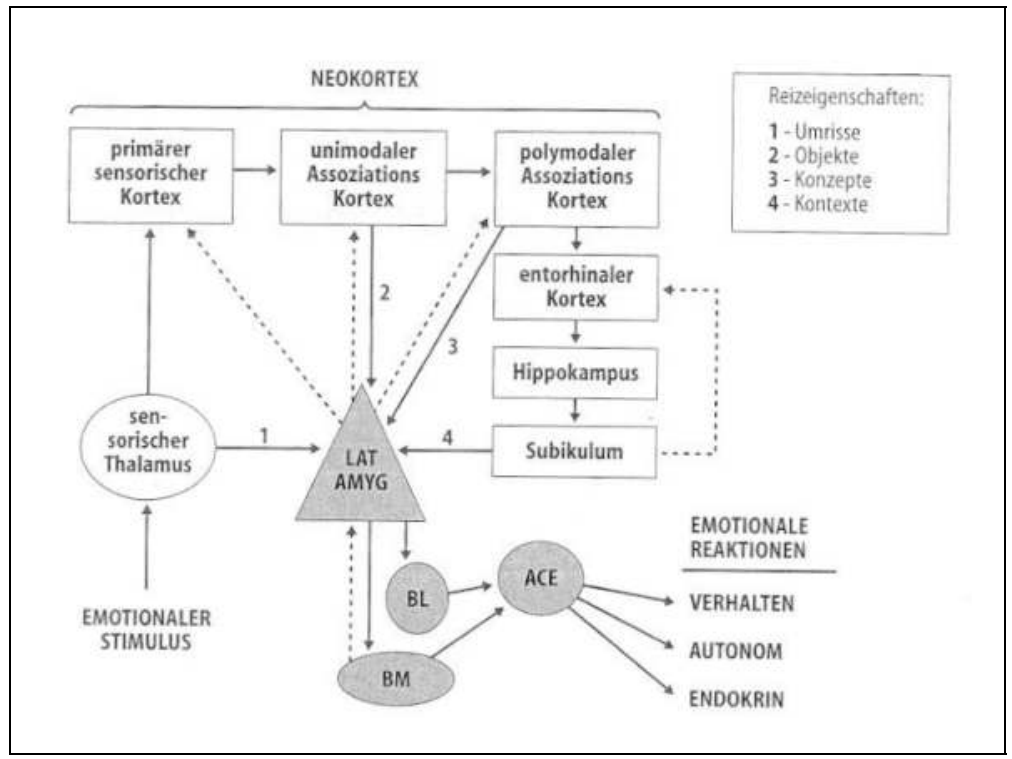

Abb. 1: Neurobiologische Mechanismen der Verarbeitung von Emotionen. „LAT AMYG" steht für laterale Amygdala, BL, BM und ACE sind weitere Teile des Nervenzellgebietes der Amygdala. Im Einzelnen handelt es sich um den basomedialen (BM), basolateralen (BL) und den zentralen Kern der Amygdala (ACE). Weitere Erläuterungen dazu im Text. Modifiziert aus: Birbaumer, Schmidt: Biologische Psychologie, Springer Verlag (1996)

Ein emotionaler Stimulus wird zunächst im sensorischen Thalamus kursorisch verarbeitet. Auf einem schnellen, aber ungenauen Weg, der keine genaue Reizdiskrimination zulässt (Weg 1 in Abbildung 1), gelangt die Information zu den lateralen Amygdala (Mandelkerne). Die Amygdala sind Hirnstrukturen, die tief im Innern des Gehirns als Teile des Emotionen verarbeitenden limbischen Systems gelegen sind. Im Bereich der Amygdala wird über weitere Verschaltungen die emotionale Reaktion mit motorischem Verhalten, Reizantwort des autonomen Nervensystems und Hormonausschüttungen programmiert. Dieser schnelle Weg der Emotionsverarbeitung spielt vor allem in lebensbedrohlichen Situationen eine Rolle und gilt als der wichtigste Mechanismus der Angstkonditionierung.

Parallel zur oben geschilderten schnellen Reizverarbeitung wird die Großhirnrinde mit eingeschaltet (Weg 2). Die primären, beispielsweise in 
der Hörrinde des Schläfenlappens gelegenen Sinnesareale decodieren das empfangene Signal und leiten es weiter an den unimodalen Assoziationskortex. Dort wird das akustische Objekt als Musik identifiziert. Eine absteigende Bahn zu den Amygdala kann dann wiederum emotionale Reaktionen auslösen. Der polymodale Assoziationskortex schließlich bindet den identifizierten Reiz in bereits bestehende Konzepte ein (Weg 3) und ermöglicht über eine Kaskade von Erregungen im Bereich des unteren Stirnhirnlappens und der Gedächtnisstrukturen die Einbeziehung des Reizkontextes in die Bewertung der emotionalen Reaktion (Weg 4).

Die oben genannten Verarbeitungswege seien erneut an dem Beispiel aus der Matthäuspassion erläutert: Beim Hören des Barrabas-Ruf werden in einem sehr schnellen Verarbeitungsweg bereits im Bereich von Hirnstamm und Mittelhirn die Lautstärkeveränderungen, die ungewohnte Harmonie und die Klangfarben des mächtigen Chorgesanges kursorisch analysiert. Die Ergebnisse dieser groben Analyse werden an die lateralen Amygdala weitergegeben und lösen dort die Kaskade emotionaler Reaktionen aus (Weg 1). Die oben genannte Orientierungsreaktion wird über diesen Weg verschaltet und führt zu der schnellen Schreckreaktion und zur Gänsehaut. Parallel dazu wird die Musik an die primäre Hörrinde und an den unimodalen Assoziationskortex im Bereich der Schläfenlappen weitergegeben. Dort erfolgt die präzise Kategorisierung. Es wird erst jetzt bewusst erkannt, dass es sich um einen mächtigen Chorklang handelt. Erneut kann dies über die lateralen Amygdala emotionale Reaktionen auslösen (Weg 2). Im polymodalen Assoziationskortex wird dann der Chorklang mit schon früher erworbenen Konzepten verknüpft. Eine grundsätzlich positive Einstellung zu Chormusik, die Einordnung des harmonischen Geschehens in den musikalischen Zusammenhang, Vorstellungen über Barockmusik im Allgemeinen werden an dieser Stelle in die emotionale Bewertung mit eingebracht (Weg 3). Schließlich kann dieses musikalische Erleben mit zahlreichen biographischen Erinnerungen verbunden werden. Die Gedächtnisstrukturen des entorhinalen Kortex, des Hippokampus und des Subiculums stellen das eben Gehörte in einen Zusammenhang mit der Vergangenheit. Erinnerungen an früher gehörte Matthäuspassionen werden wach, liebe Menschen, mit denen das Werk damals gemeinsam gehört wurde, die Orte der Aufführung, und vergangene Lebensgefühle kommen in das Gedächtnis. Literarisch hat Marcel Proust in seinem Roman Auf der Suche nach der verlorenen Zeit diese durch Musik ausgelösten Erinnerungsstürme meisterhaft geschildert. 
Wichtig ist, dass die in Abbildung 1 gezeigten neuronalen Bahnen teilweise auch in umgekehrter Richtung verlaufen. Die lateralen Amygdala können den primären sensorischen Kortex, den unimodalen Assoziationskortex und den polymodalen Assoziationskortex beeinflussen. Das heißt, unsere bewusste Wahrnehmung ist nie objektiv, sondern wird durch die in den Amygdala programmierten Emotionen affektiv eingefärbt. Die „rosarote Brille“, durch die alles gesehen wird, drückt diesen Vorgang umgangssprachlich aus.

\section{Musik als emotionale Kommunikation}

Nachdem die grundsätzlichen Vorgänge der Emotionsneurobiologie an einem Beispiel aus der Matthäuspassion erörtert wurden, soll im Folgenden spezifischer auf die Emotionen beim Musikhören eingegangen werden. Eine mögliche Betrachtungsweise ist, Musik als ein Modell akustischer Kommunikation von Emotionen aufzufassen. In Abb. 2 ist dies auf vereinfachte Weise dargestellt.

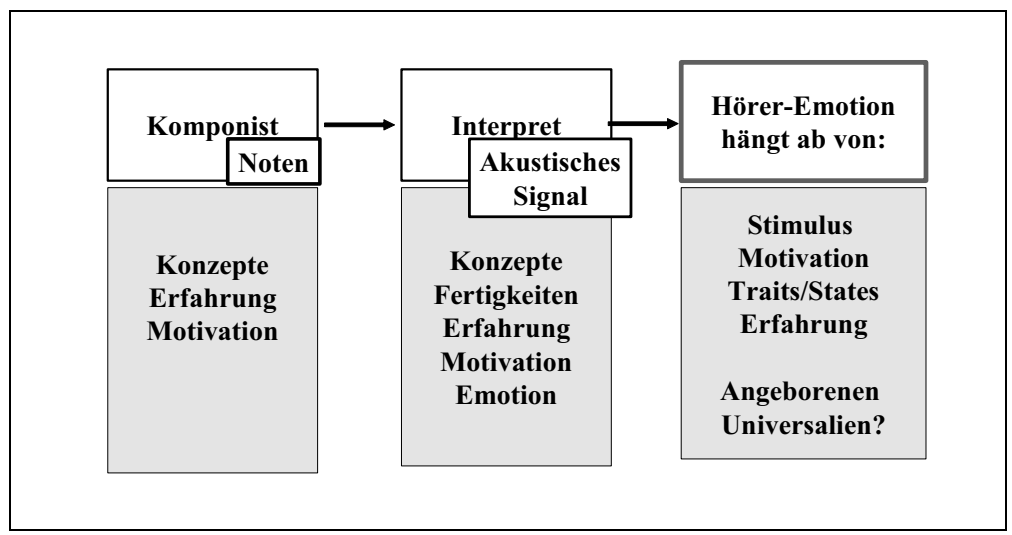

Abb. 2: Komponieren und Musizieren als Kommunikation von

Emotionen. Weitere Erläuterungen im Text.

Auf der linken Seite im Modell befindet sich der Komponist, der neuartige Repräsentationen von Musik erzeugt und im Symbolsystem der Notenschrift dokumentiert. Der Interpret liest diese Noten und produziert akustische Signale, die beim Hörer eine Emotion auslösen. Die kompositorische Leistung hängt von den künstlerischen Konzepten ab, die der Komponist verfolgt. Kompositorische Erfahrung, der affektive Zustand 
beim Komponieren und die Motivation des Komponisten sind weitere Faktoren, von denen die Komposition beeinflusst wird. Auf der Seite des Interpreten hängt dessen Interpretation des Musikstückes ebenfalls von seinen künstlerischen Konzepten ab, darüber hinaus von seinen technischen Fertigkeiten, von seiner Erfahrung, seiner Motivation und von seiner Emotion im Moment der Interpretation. Signaltheoretisch gesehen kann der Interpret also auch als Stör- oder Rauschfaktor betrachtet werden, der die Botschaft des Komponisten auf dem Weg zum Hörer nach bestimmten Gesichtspunkten aktiv filtert.

Beim Hörer hängt die Art der erzeugten Emotion nicht nur davon $a b$, was er hört. Wichtig für die emotionale Bewertung ist die momentane Motivation. Darüber hinaus spielen früh erworbene, eventuell angeborene Persönlichkeitsmerkmale mit Sicherheit eine wesentliche Rolle. Des Weiteren sind Hörerfahrung und Bekanntheitsgrad der gehörten Musik für das emotionale Erleben von Bedeutung. Schließlich kann spekuliert werden, dass angeborene emotionale Reaktionsmuster auf bestimmte akustische Signaleigenschaften Emotionen auslösen können. Die neurobiologischen Grundlagen dieser Höreremotionen sind noch so gut wie unbekannt.

Um darüber etwas präziseres Wissen zu erhalten, haben wir in einem Experiment untersucht, ob sich die Hirnaktivierung bei angenehm und unangenehm empfundener Musik unterscheidet. Ausgangspunkt waren dabei Versuche in der visuellen Modalität, die bereits von Gerhard Roth $^{10}$ vorgestellt wurden. Mit Bildern, die negative oder positive Valenzurteile auslösten, konnte gezeigt werden, dass je nach Valenz unterschiedliche neuronale Netzwerke beteiligt sind.

Diese Versuchsidee wurde auf die Musik übertragen. Als zu Grunde liegendes Emotionsmodell wurde ein Valenz-Arousal Model1 ${ }^{11}$ herangezogen. In diesem Modell wird versucht, die verschiedenen Emotionen in einem zwei-dimensionalen Raum zu beschreiben, nämlich einerseits in der Dimension „Valenz“ (mag ich - mag ich nicht) und in der Dimension „Arousal“ (regt mich an - beruhigt mich). Positive Valenz und hohes Arousal charakterisieren die Emotion „Fröhlichkeit“, negative Valenz und hohes Arousal charakterisieren die Emotion „Ärger“, negative Valenz und niedriges Arousal charakterisieren die

10 Vgl. Roth, Gerhard: „Wahrnehmung - Abbildung oder freies Konstrukt“ in diesem Band, S. 15-33.

11 Russell, John: „Measures of emotion“, in: Robert Plutchik/Henry Kellerman (Hrsg.): Emotion: Theory research and experience, 4 (1989), S. 81-111. 
Emotion „Trauer“, positive Valenz und niedriges Arousal charakterisieren „Schläfrigkeit“.

Hinsichtlich der Hirnsubstrate dieser Emotionen existieren zwei Theorien nebeneinander. Die Valenz-Theorie besagt, dass unterschiedliche Emotionen in verschiedenen Hirnhemisphären verarbeitet werden, nämlich positive Emotionen in der linken, Negative in der rechten Hirnhälfte. Wichtiger moderner Vertreter der Valenztheorie ist Davidson. ${ }^{12}$ Dem gegenüber steht die Hemisphärentheorie, die besagt, dass alle Emotionen, unabhängig von ihrer Valenz in der rechten Hirnhälfte verarbeitet werden. Sie wird von der Mehrheit der Neuropsychologen bevorzugt. ${ }^{13}$ Um festzustellen, welche der beiden Theorien zutrifft, variierten wir im Experiment systematisch den Parameter Valenz beim Musikhören. Der Parameter Arousal wurde möglichst konstant gehalten um eindeutige Aussagen bezüglich der Valenz zu erhalten. ${ }^{14}$

Sechzehn jugendliche Versuchspersonen im Alter zwischen 12 und 14 Jahren mit vergleichbarer Musikerfahrung und ähnlichem soziobiographischem Hintergrund hörten 120 Musikausschnitte und 40 Umweltgeräusche, die jeweils 15 Sekunden dauerten. Alle Reize besaßen nach Vortests eine ähnliche Arousal-Dimension und wurden als mittelgradig aktivierend empfunden. Die Musikausschnitte hatten wir nach den Vortests so zusammengestellt, dass 80 Hörbeispiele eher positive, 80 eher negative Emotionen erwarten ließen. Nach jedem Musikstück hatten die Versuchspersonen die Aufgabe, die Musikstücke auf einer 5 Punkte Skala nach Schulnoten zu bewerten.

12 Davidson, Richard: „Cerebral asymmetry, emotion and affective style“, in: ders./Hugdahl, Kenneth (Hrsg.): Brain asymmetry, Bradford 1995, S. 361387.

13 Blood, Anne/Zatorre, Robert: „Intensely pleasurable responses to music correlate with activity in brain regions implicated in reward and emotion", in: Proceedings of the National Academy of Science, 98 (2001), S. 1181811823.

14 Altenmüller, Eckart/Schürmann, Kristian/Lim, Vanessa/Parlitz, Dietrich: „Hits to the left - Flops to the right. Different emotions during music listening are reflected in cortical lateralisation patterns", in: Neuropsychologia, 40 (2002), S. 2242-2256. 


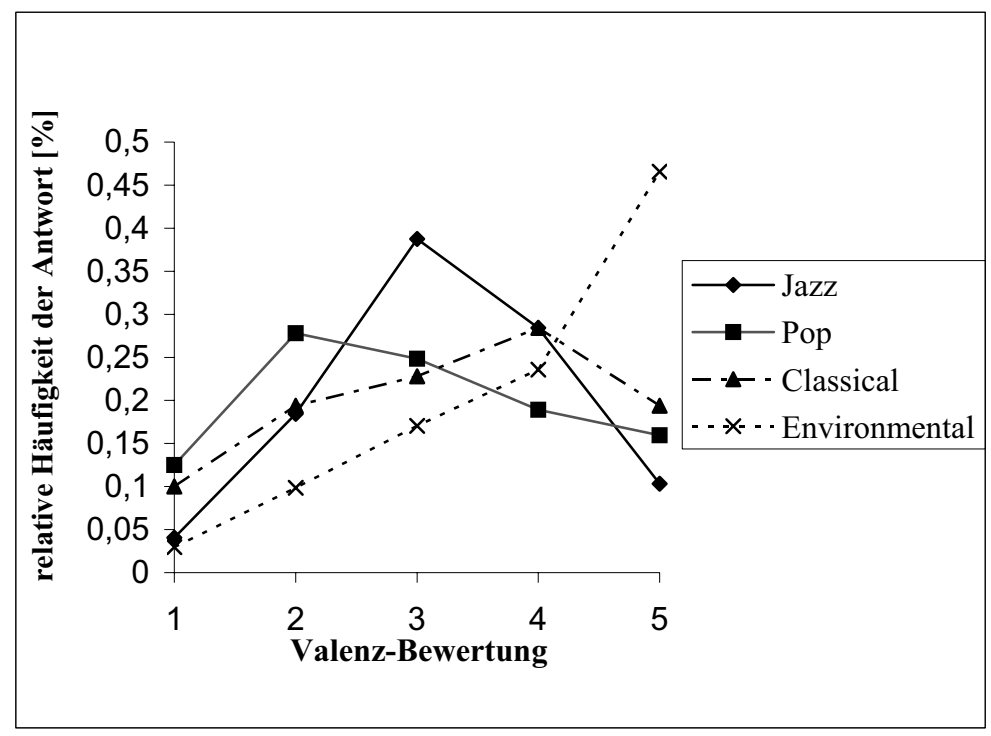

Abb. 3: Valenzurteile der Jugendlichen für die vier unterschiedlichen Kategorien Jazz, Pop, klassische Musik und Umweltgeräusche. Die Jugendlichen hatten die Aufgabe, ihre Bewertung auf einer fünf Punkte Skala anzugeben: 1.) Mag ich sehr 2) mag ich 3.) unentschieden 4.) mag ich nicht 5.) mag ich überhaupt nicht. Weitere Erläuterungen im Text. (Abbildung modifiziert aus Altenmüller et al. 2002).

In Abbildung 3 sind die Bewertungen der Jugendlichen dargestellt. Grundsätzlich wurde dissonante Musik negativer bewertet als konsonante Musik. Aber auch je nach Musikstil fallen die Benotungen unterschiedlich aus. Auf der rechten Seite sind die Bewertungshäufigkeiten für $\mathrm{Mu}-$ sikstücke aufgetragen, die die Jugendlichen weniger mögen (Noten 4 und 5), auf der linken, für diejenigen, die sie mehr mögen (Noten 1 und 2). Exzerpte aus klassischer Musik führen zu einer Rechtsverschiebung, Jazzmusik erzeugt eine symmetrische Verteilung, Popmusik eine eher positive Bewertung. Umweltgeräusche erzeugen häufiger negative $\mathrm{Be}$ wertungen.

Die Hirnaktivierung wurde anschließend bei jedem Jugendlichen je nach individueller Bewertung der Musikstücke getrennt für positiv und negativ bewertete Musik ausgewertet. Es zeigte sich, dass positiv bewertete Musik eine stärkere linkshemisphärische Aktivierung vor allem im Bereich der Stirnhirnrinde erzeugte, negative eine stärker beidseitig 
symmetrische Aktivität. In Abb. 4 sind die Mittelwerte der Hirnaktivierungen getrennt für Mädchen (oben) und Jungen (unten) dargestellt.

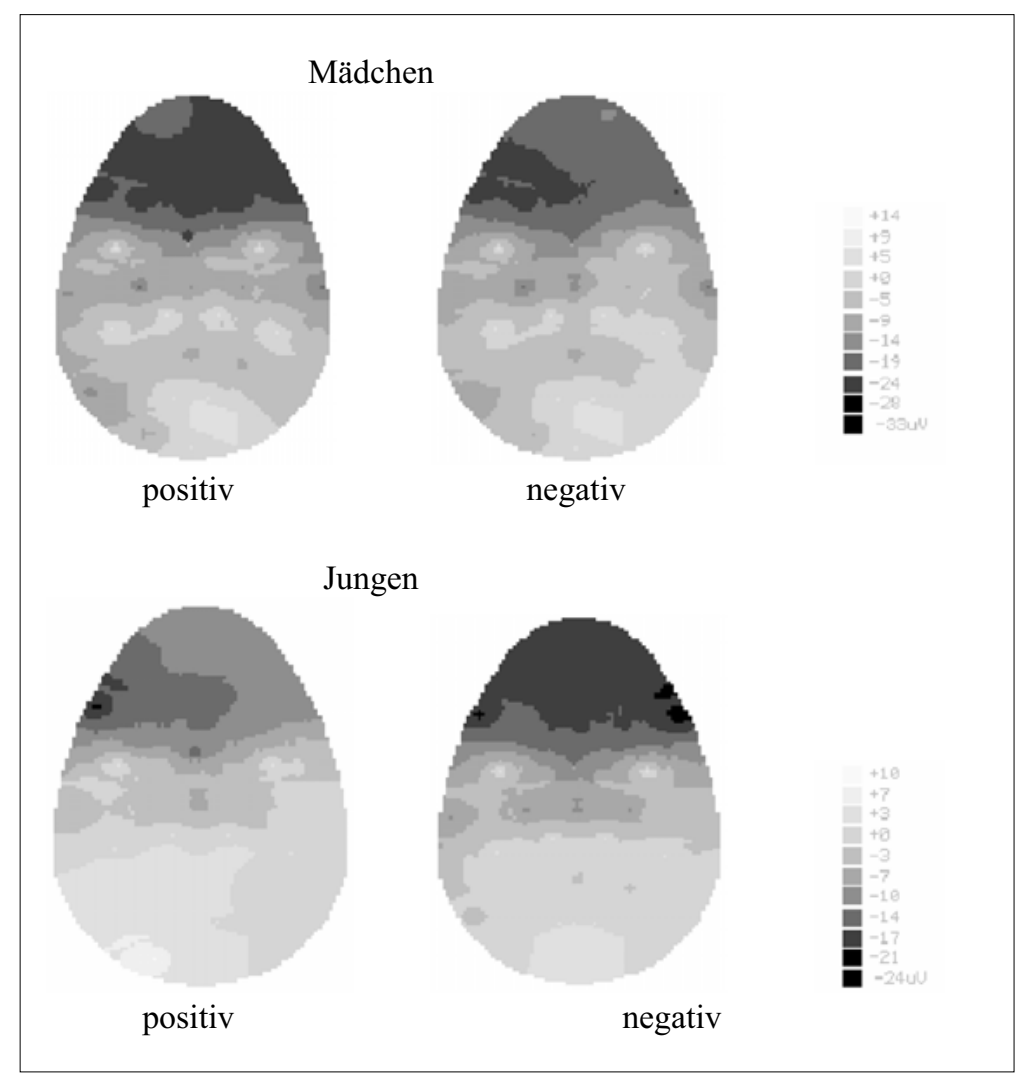

Abb. 4: Ergebnisse der Gleichspannungs-EEG-Messung beim Hören von positiv (links) oder negativ (rechts) bewerteten Musikausschnitten.

Gemittelte Aktivierungen über jeweils acht weibliche (oben) und acht männliche (unten) Jugendliche. Blick von oben auf das Gehirn. Die Stirn wäre in den topographischen Darstellungen jeweils oben. Die linke Hirnhälfte ist jeweils auf der linken Seite der einzelnen Kopfdiagramme aufgetragen, die rechte Hirnhälfte rechts. Weitere Erläuterungen im Text. Abb. Modifiziert aus Altenmüller et al. 2002.

Man sieht hier von oben auf die Gehirne der Jugendlichen. Links aufgetragen sind die Aktivierungsmuster während der positiv bewerteten $\mathrm{Mu}-$ sikstücke, rechts während der negativ bewerteten. Je stärker die Hirnre- 
gionen aktiviert sind umso dunkler ist die Färbung. Als wesentliches Ergebnis der Untersuchung kann festgehalten werden, dass positive Bewertung der Musikausschnitte mit einer stärkeren Asymmetrie der Hirnaktivität zu Gunsten der linken Hemisphäre bei Mädchen und bei Jungen einhergeht.

Zusammenfassend sprechen diese Daten für die Valenz-Hypothese. Nachteil des Experiments ist, dass möglicherweise bei der Bewertung der Musikstücke die Schüler mehr angelernten Konzepten vertrauten als ihren wahren Emotionen. Valenzurteile müssen ja nicht notwendigerweise den gefühlten Emotionen entsprechen.

\section{Gänsehaut und Kloß im Hals: Starke Emotionen beim Musikhören}

Aus diesem Grund suchen wir derzeit nach objektiven Parametern, die über die gefühlte Emotion Auskunft geben können. Dafür kann man sich ein Phänomen zunutze mache, das als SEM (,Strong Emotions in Music“) bekannt ist. ${ }^{15}$ Es handelt sich um starke Musikerlebnisse, die zu Reaktionen des autonomen Nervensystems führen. Sie werden als „Gänsehaut“, „Tränen“, „Kloßgefühl im Hals“, „Flattern im Bauch“ oder „Herzrasen“ beschrieben. Derartige starke emotionale Erlebnisse und entsprechende autonome Antworten beim Musikhören sind wohl ein recht häufiges Phänomen. Nach Panksepp ${ }^{16}$ erleben $86 \%$ amerikanischer Studenten dieses Phänomen regelmäßig, Sloboda ${ }^{17}$ fand es bei $90 \%$ erwachsener Studienteilnehmer und Goldstein ${ }^{18}$ bei $90 \%$ der Musikstudenten, aber nur bei $53 \%$ der untersuchten Krankenhausangestellten. Nach unseren aktuellen Ergebnissen treten allerdings SEMs bei deutschen Hörern deutlich seltener auf (persönliche Mitteilung Frederik Nagel und Oliver Grewe). Offensichtlich besteht nach den drei oben zitierten, allerdings eher explorativen Studien kein Unterschied zwischen den Geschlechtern. Unklar blieb bisher, ob es sich um konditionierte oder unkonditionierte Reaktionen handelt. Konditionierungs-Prozesse scheinen

15 Gabrielson, Alf: „Emotions in strong experiences with music“, in: Juslin, Patrique/Sloboda, John (Hrsg.): Music and emotion: Theory and research, Oxford 2001, S. 431-452.

16 Panksepp, Jaak: „The emotional Sources of ,Chills“ induced by music“, in: Music Perception, 13 (1995), S. 171-207.

17 Sloboda, John: „Music Structure and Emotional Response: Some Empirical Findings“, in: Psychology of Music, 19 (1991), S. 110-120.

18 Goldstein, Albert: „Thrills in response to music and other stimuli“, in: Physiological Psychology, 8 (1980), S. 126-129. 
jedoch eine Rolle zu spielen, da nach Panksepp ${ }^{19}$ die Wahrscheinlichkeit für Gänsehaut bei schon bekannten Musikstücken höher ist als bei völlig unbekannten. Allerdings tritt Gänsehaut auch regelmäßig bei vollkommen unbekannter Musik auf, was gegen die Annahme einer ausschließlich konditionierten Antwort spricht. Die SEMs auslösenden musikalisch-strukturellen Parameter sind von Sloboda ${ }^{20}$ genauer beschrieben worden. Er untersuchte an einer Stichprobe von 83 musikalisch ausgebildeten Probanden ausschließlich SEMs bei klassischer Musik. Für die Auslösung von Gänsehaut waren die wesentlichen musikalisch-strukturellen Parameter neue und unerwartete harmonische Wendungen. Tränen und ein Kloßgefühl im Hals traten bei abfallenden Quintfall-Sequenzen und melodischen Vorhalten auf. Panksepp ${ }^{21}$ brachte die SEMs mit einem eher traurigen, ,zartbitteren“ Gefühl von sozialem Verlust und Sehnsucht in Verbindung und identifizierte als akustische Auslöser vor allem das Anschwellen der Lautstärke und das Herauslösen eines Instrumentes aus dem Gesamtklang. Panksepp deutet diese Befunde als Hinweis auf einen evolutionären Ursprung der SEMs in den Trennungsrufen der Primaten. Die Isolation des einzelnen Instrumentes aus dem Gesamtklang wird als soziale Metapher der Vereinsamung aufgefasst. Diese metaphorisch soziale Komponente scheint auch nach unseren Erfahrungen ein wichtiger Faktor zur Auslösung von SEMs zu sein. So kann die starke emotionale Reaktion beim Hören des Barrabas Ruf aus der Matthäuspassion sehr gut mit sozialen Gefühlen in Verbindung gebracht werden. Musik kann hier also als universal verstandene Metapher für soziale Konstellationen des Einzelnen im Verhältnis zur Gruppe betrachtet werden.

Interessant ist die Neurobiologie der SEMs. Bereits Goldstein ${ }^{22}$ vermutete, dass die Vermittlung von SEMs neurobiologisch durch das endogene Opiatsystem erfolgt. Bei drei von zehn Versuchspersonen war die Gänsehaut nach Injektion des Opiatantagonisten Naloxon signifikant reduziert. Eine neue Hirnaktivierungsstudie unterstützt diese Befunde. Anne Blood und Robert Zatorre ${ }^{23}$ erfassten durch Messung der Zunahme der lokalen Hirndurchblutung die Großhirnaktivierung während Probanden an selbst mitgebrachten Musikstücken SEMs erlebten. Unter den Stücken befanden sich Werke, die bereits in der Studie von Sloboda ${ }^{24}$ genannt worden waren, etwa Rachmaninoffs zweites Klavierkonzert in d-

19 Panksepp (wie Anm. 16).

20 Sloboda (wie Anm.17).

21 Panksepp (wie Anm. 16).

22 Goldstein (wie Anm.18).

$23 \mathrm{Bood} /$ Zatorre (wie Anm.13).

24 Sloboda (wie Anm. 17). 
moll oder Barbers Adagio für Streicher. Die Hirnaktivierungsmuster während der SEMs ergaben Aktivierungen im Bereich des Mittelhirns, der Basalganglien, des Stirnhirns, des inneren Schläfenlappens und der Amygdala. Alle diese Hirnstrukturen werden zu dem limbischen System gezählt, das Emotion, Motivation und Selbstbelohnung programmiert. Dieselben Strukturen sind auch aktiv, wenn Probanden Kokain einnehmen oder in sexuelle Erregung geraten.

\section{Musik als Sprache der Gefühle?}

Worin liegen nun die evolutionären Ursprünge dieser emotionalen Wirkung von Musik und warum hat sich Musik als Kommunikationssystem bis in unsere Zeit erhalten? In Abb. 5 schlagen wir ein nach Brown ${ }^{25}$ weiterentwickeltes hypothetisches Modell zur Beantwortung dieser Fragen vor.

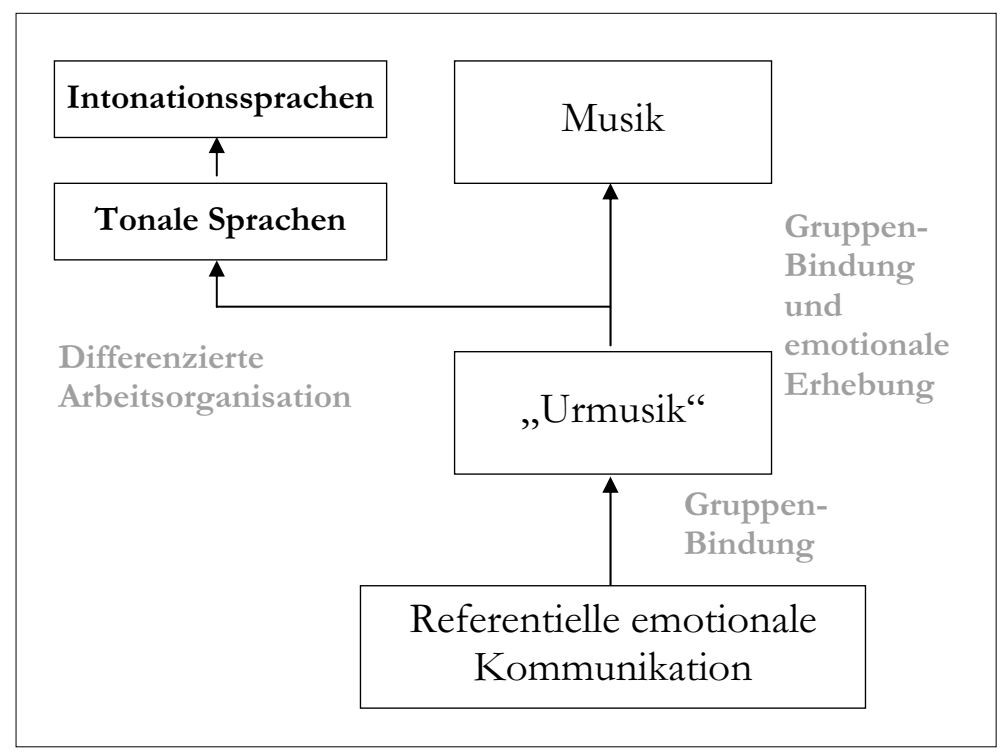

Abb. 5: Nach Brown (2000) modifiziertes Modell, das die Entwicklung der Musik und der Sprache verdeutlicht. Nähere Erläuterungen siehe Text.

25 Brown, Steven: „The ,Musilanguage'-Model of Music Evolution“, in: Wallin, Nils/Merker, Björn/Brown, Steven (Hrsg.): The origins of music, Cambridge 2000, S. 271-300. 
Viele in Gruppen lebende Säugetiere verfügen über eine referentielle emotionale Kommunikation. Murmeltiere beispielsweise warnen ihre Artgenossen bei Angriffen durch einen Greifvogel aus der Luft mit anderen Rufen als bei Angriffen auf ebener Erde durch einen Fuchs. Ein derartiges innerartliches referentielles Kommunikationssystem verbessert die Überlebenschancen der Gruppe und kann auch in der Vermittlung von Emotionen für Gruppenziele eingesetzt werden. Möglicherweise haben frühe Hominiden über eine Form der gesungenen Urmusik eine stärkere Gruppenbindung gegenüber konkurrierenden, nicht singenden Primatenarten erzielt. Diese ersten Formen von Musik dienten dann nicht nur der Gruppenorganisation sondern erzeugten bei anstrengender körperlicher Arbeit Trance und „Flow“ und ermöglichten dadurch Höchstleistungen - ähnlich wie es heute bei tanzenden Jugendlichen in Diskotheken beobachtet werden kann. In zunehmendem Ausmaß benötigte jedoch die differenzierte Arbeitsorganisation eine effizientere Kommunikation. Anthropologen vermuten, dass zunächst Gebärdensprachen entwickelt wurden, aus denen sich dann die gesprochene Sprache ausdifferenzierte. Dafür spricht, dass die Broca-Sprachregion der linken Stirnhirnrinde nicht nur beim Sprechen, sondern auch bei symbolischen Gesten aktiviert wird. ${ }^{26}$ Strittig ist derzeit, ob zunächst tonale Sprachen entstanden, in denen Tonhöhen Wortbedeutungen verschlüsselten. Möglicherweise sind die Intonationssprachen parallel entstanden. Sie setzen Tonhöhenkonturen und nicht absolute Tonhöhen für die Kodierung ein. Musik wurde als zweites Kommunikationssystem beibehalten, um Gruppenprozesse zu fördern und um über Glückserlebnisse das harte Leben erträglicher zu machen. Marcel Proust hat die einzigartige Rolle der Musik und ihr Verhältnis zur Sprache meisterhaft in einer Passage seines Romans Auf der Suche nach der Verlorenen Zeit in Worte gefasst. Es ist eine Szene, in der der Erzähler ein bewegendes Konzert verlässt und ihn mehrere alte Bekannte im Foyer des Konzertsaales ansprechen:

[...] Aber was bedeuteten ihre Worte, die wie jede nur am äußern haftende menschliche Rede mich so gleichgültig ließen, verglichen mit dem himmlischen musikalischen Thema, mit dem ich mich zuvor unterhalten hatte? Ich fühlte mich wahrhaft wie ein Engel, der, aus dem Rausch des Paradieses herabgestürzt, in die trivialste Wirklichkeit fällt. Und ich fragte mich, ob nicht ...... die Musik das einzige Beispiel dessen sei, was - hätte es keine Erfindung der Sprache, Bildung von Wörtern, Analyse der Ideen gegeben - die

26 Corina, Dorothee/McBurney, Steven: „The neural representation of language in users of American Sign Language“, in: Journal of Communication Disorders, 34 (2001), S. 455-71. 
mystische Gemeinschaft der Seelen hätte werden können. Sie ist wie eine Möglichkeit, der nicht weiter stattgegeben wurde; die Menschheit hat andere Wege eingeschlagen, die der gesprochenen und geschriebenen Sprache. Aber diese Rückkehr zum Nichtanalysierbaren war so berauschend, daß mir beim Verlassen des Paradieses die Berührung mit mehr oder weniger klugen Menschen außerordentlich banal erschien. ${ }^{27}$

\section{Danksagung}

Ganz herzlich möchte ich meinen Kollegen Elke Zimmermann, Sabine Schmidt und Reinhard Kopiez für zahlreiche Anregungen und fruchtbare Diskussionen zu den Themen des Artikels danken. Meinen Mitarbeitern im IMMM, insbesondere den „Emotionsprojektlern“ Oliver Grewe, Frederik Nagel und Katja Goydke sei ebenfalls für zahlreiche produktive, oft auch emotionale Emotionsdiskussionen gedankt. Klaus-Ernst Behne hat mich bei der Durchführung der Experimente und bei der Auswahl der getesteten Musikstücke mehrfach sehr gut beraten. Die Valenz-Experimente konnten nur durch den unermüdlichen Einsatz von Kristian Schürmann und die Unterstützung von Dietrich Parlitz realisiert werden. Der Deutschen Forschungsgemeinschaft danke ich für die Möglichkeiten, experimentell die Wirkung von Musik auf die Emotionen untersuchen zu können.

27 Proust, Marcel: Auf der Suche nach der verlorenen Zeit, Frankfurt a.M. 1979, S. 3096-3097. 

Karl Clausberg

\title{
„EIN MIKROSKOP FÜR DIE ZEIT“.
}

\author{
WAS BENJAMIN UND KLAGES, EINSTEIN \\ UND DAS KINO DEN FERNEN STERNEN \\ VERDA N KEN
}

Umbrüche in den menschlichen Vorstellungs- und Medienwelten werden derzeit mit Vorliebe auf neue technische Errungenschaften zurückgeführt. Die Geschichte der optischen Medien - camera obscura, Photographie, Kino, Fernsehen etc. - scheint mittlerweile als Paradebeispiel für solche Betrachtungsweisen fest etabliert. Aber wie vertragen sich solche Fortschrittsperspektiven mit der ,Natur' des Menschen, die sich irgendwie durchs Flussbett anthropologischer Veranlagungen, neurobiologischer Determiniertheiten zu zivilisatorischen Höhenflügen durchgerungen hat? Dass die Wechselwirkungen zwischen wissenschaftlichen Entdeckungen, technischen Neuerungen und extrapolierender Phantasie oft sehr komplex waren und sich nicht in einfache Ursache-Folge-Sequenzen mit der Technik als Schrittmacher bzw. Genetik als Trimmung und Bremse auflösen lassen, möchte ich anhand eines kleinen, zunächst anonym publizierten Textes eines Berliner Astronomieliebhabers aus der Mitte des 19. Jahrhunderts demonstrieren, dessen beträchtliche Auswirkungen im Titel dieses Beitrags andeutend abgesteckt sind.

Die auszugsweise Präsentation und Analyse dieser bislang unbeachtet gebliebenen Schrift von Felix Eberty und ihrer Wirkungsgeschichte kann exemplarisch dazu dienen, den exklusiv evolutionsbiologischen und kognitiv-neuronal begründeten Kulturmodellen, die teilweise recht ungeniert ihre allumfassenden Erklärungsansprüche anmelden, etwas Wind aus den Segeln zu nehmen. Wenn die hier skizzierten $\mathrm{Zu}-$ sammenhänge auch nur halbwegs richtig erfasst sind, dann ist gerade in Schlüsselbereichen der menschlichen Vorstellungsleistungen mit größeren Freiheitsgraden, Eigenwilligkeiten und überraschenden Wendungen von individueller Einsichtsfähigkeit und kollektiv kultureller Selbstzurichtung zu rechnen, als sie von manchen Vertretern der neuen Leitwissenschaften vorgesehen werden. 


\section{Albert Einstein}

Im Jahre 1923 erschien in Berlin ein kleines Bändchen mit dem Titel Die Gestirne und die Weltgeschichte/Gedanken über Raum, Zeit und Ewigkeit von einem gewissen Felix Eberty. ${ }^{1}$ Wie aus der Vorrede des Herausgebers Gregorius Itelson ${ }^{2}$ und dem beigefügten Vorwort des Verfassers zur Zweitauflage von 1874 zu entnehmen war, hatte die kleine, ursprünglich 1846/47 veröffentlichte Schrift ein bemerkenswertes Schicksal hinter sich: Die anonyme deutsche Erstfassung war in englischer Raubübersetzung weltweit verbreitet worden, und der renommierte Sekretär der Britischen Royal Astronomical Society, Richard Proctor, hatte sich die spekulativen Perspektiven in seinem Bestseller Other Worlds than Ours (1870ff.) zu eigen gemacht. Zudem seien die Ebertyschen Ideen von Camille Flammarion in dessen „geschwätzigem“ Buche Lumen ohne Quellenangabe weit ausgesponnen worden, so Herausgeber Itelson nicht ohne Ranküne. Auch noch auf anderen Wegen waren Ebertys astronomische Phantasien seit einem Dreivierteljahrhundert in Umlauf gekommen. Der Berliner Neudruck war ein später Nachzügler; er präsentierte noch einmal den ursprünglichen Textbrocken, der in einer von ihm ausgelösten Lawine schließlich wieder nach oben getragen worden war.

Der Name des Autors dürfte in den zwanziger Jahren gebildeten Lesern noch durchaus vertraut gewesen sein: Eberty hatte nicht nur Biographien Walter Scotts und Lord Byrons, sondern auch eine siebenbändige Geschichte des preußischen Staates verfasst. Die kleine Astronomie-Schrift muss auf zusätzliches Interesse gestoßen sein; jedenfalls wurde das Bändchen 1925 - neben einer ebenfalls nachgedruckten Autobiographie $^{3}$ - nochmals unverändert aufgelegt. ${ }^{4}$ Sehr auffällig war auch damals schon die Titelunterschrift, die darauf hinwies, dass kein geringerer als Albert Einstein, der Physik-Nobelpreisträger von 1921, die Einleitung beigesteuert hatte. „Dies Büchlein, von einem originellen, geistreichen Menschen geschrieben, entbehrt nicht des aktuellen Interesses“, so Einsteins erste Worte vom 5. Juni 1923.

Denn es zeigt auf der einen Seite kritischen Geist gegenüber dem überkommenen Zeitbegriff; auf der anderen Seite zeigt es, vor wel-

1 Eberty, Felix: Die Gestirne und die Weltgeschichte. Gedanken über Zeit, Raum und Ewigkeit. Mit einer Einleitung von Albert Einstein, Berlin 1923.

2 Zur Person Itelsons vgl. Buck, Otto: „Gregorius Itelson“; in: Kant-Studien, 1926, S. 428-430.

3 Eberty, Felix: Jugenderinnerungen eines alten Berliners, Berlin 1925.

4 Eberty, Felix: Die Gestirne und die Weltgeschichte. Gedanken über Zeit, Raum und Ewigkeit. Mit einer Einleitung von Albert Einstein, Berlin 1925. 
chen eigentümlichen Folgerungen uns die Relativitätstheorie rettet, der doch so vielfach gerade der bizarre Charakter ihrer Folgerungen zum Vorwurf gemacht wird.

Sollte man aus dieser Formulierung, neben dem Hinweis auf aktuelle Querelen um deutsche oder jüdische Physik ${ }^{5}$, am Ende auch herauslesen, dass Einstein die wesentlichen Inhalte des Büchleins schon lange kannte und womöglich durch sie mit zum grundlegenden Konzept der Relativitätstheorie angeregt worden ist? Solche Spekulationen sind keineswegs aus der Luft gegriffen, denn Einstein hat in seinen Jugenderinnerungen ausdrücklich ein populärwissenschaftliches Sammelwerk aus dem 19. Jahrhundert erwähnt, in dem die Ebertysche Schrift seitenlang referiert wurde. „Auch hatte ich das Glück“, schrieb Einstein 1949 in seinen Autobiographischen Notizen, ,die wesentlichen Ergebnisse und Methoden der gesamten Naturwissenschaft in einer vortrefflichen populären, fast durchweg aufs Qualitative sich beschränkenden Darstellung kennen zu lernen (Bernsteins naturwissenschaftliche Volksbücher, ein Werk von fünf oder sechs Bänden), ein Werk, das ich mit atemloser Spannung las. “6

Diese Ausgabe der Bernsteinschen Volksbücher hatte ein Freund der Familie, der Medizinstudent Max Talmud (nach der Emigration: Talmey), 1889 dem elfjährigen Einstein gegeben, um dessen Wissbegier und eigenständige Studien zu fördern. Der Quartaner des Münchener Luitpoldgymnasiums sei zutiefst beeindruckt gewesen, notierte Talmey 1932 in seinem Rückblick auf die, formative Periode des Erfinders der Relativitätstheorie‘?

\section{Aaron Bernstein}

Aaron Bernstein (1812-1884), Begründer der Berliner Volkszeitung, hatte seine Naturwissenschaftlichen Volksbücher seit 1855 in unzähligen erweiterten Auflagen publiziert. Die fünf- oder sechsbändige Ausgabe, die Jugendfreund Talmud dem Schüler Einstein ans Herz legte, war vermutlich eine jener neuerlich verbesserten und vermehrten Versionen der

5 Schönbeck, Charlotte: „Albert Einstein und Philipp Lenard. Antipoden im Spannungsfeld von Physik und Zeitgeschichte"; in: Schriften der Mathematisch-Naturwissenschaftlichen Klasse der Heidelberger Akademie der Wissenschaften, 8 (2000).

6 Einstein, Albert: Autobiographical Notes [1949], hrsg. v. Paul Arthur Schilpp, Illinois 1991, S. 12 u. S. 14.

7 Talmey, Max: The Relativity Theory Simplified/And the Formative Period of ist Inventor, New Bedford, Mass. 1932, S. 159ff., spez. S. 162. 
vierten Auflage, die ab 1873/74 erschienen. Im einundzwanzigstem Teil war ein Kapitel über Die Unendlichkeit und die Naturwissenschaft $\mathrm{zu}$ finden, das dem jungen Einstein sicherlich in Erinnerung geblieben ist, denn es brachte fundamentale Perspektiven und Probleme der Astronomie und Kosmologie zur Sprache: „Der kühne Fernblick William Herschels, der zuerst [mit seinem berühmten Riesenteleskop] in den Raum der Fixsternwelt eindrang, hatte dem staunenden Geist der Menschheit ein neues Weltbild aufgeschlossen“, war da zu lesen gewesen; und weiter:

Wir erblicken zwei Gestirne, sobald sie nur in verschiedenen Entfernungen von uns existiren, auch nothwendig in verschiedenen Alters-Epochen, selbst wenn beide in einem und demselben Augenblicke entstanden wären. Der Raumunterschied bedingt für unsere Wahrnehmung auch zugleich den Zeitunterschied. Was wir aus unendlichen Räumen zu sehen bekommen, zeigt uns auch zugleich Gebilde aus unendlichen Zeiten.

William Herschel habe sich auch nicht gescheut, so Bernstein anschließend, die noch schärfere Konsequenz dieses Gedankens auszusprechen: Ließen sich die Beobachtungen noch weiter vorantreiben, ,so würde ein Rundblick am Himmel zu einem Rundblick in die Geschichte der Weltenentstehung, in den ferneren Tiefen des Raumes und der Zeit werden.“ ${ }^{\circ}$

Nach solchen Prämissen setzte Bernstein zum Loblied auf einen Unbekannten an, den er nicht zögerte, mit Herschel in einem Atemzuge zu nennen: Über viele Jahrzehnte hätten die tiefen Gedanken des Teleskop-Pioniers brachgelegen, ohne fruchtbringend Wurzeln zu schlagen; aber dann:

Im Jahre 1840 spann ein freier, logischer Kopf in Deutschland in einigen anonym erschienenen, kleinen Schriften diese Grundgedanken Herschel's zu einem sinnigen Gedankenspiel weiter aus. Ist es richtig, - schloß dieser - daß das Licht uns aus weiten Fernen die Botschaft der Vorgänge und Zustände erst nach Jahren zuträgt und uns so Gebilde der Vergangenheit zeigt, so ist nicht minder auch das Umgekehrte wahr. Auch was auf unserer Erde gegenwärtig vorgeht, wird die Lichtbotschaft erst nach längerer Zeit in die Ferne hintragen. [...] Auf einem Fixsterne, dessen Licht erst in zehn Jahren zu uns dringt, wird man die Vorgänge auf unserem Erdenrund auch erst zehn Jahre nach dem Ereigniß wahrnehmen. Was ein Jahrzehnt hinter uns liegt, ist für den dortigen Blick noch Gegenwart. Denken wir uns noch weiter hinaus in den Welten-

8 Bernstein, Aaron: Naturwissenschaftliche Volksbücher, Wohlfeile Gesammt-Ausgabe, 4. Abdruck, 5. Bd., 21. Theil, Berlin o.J. [1873-74], S. 76-111, spez. S. 99. 
raum, so vergegenwärtigt sich noch immer in weiteren und weiteren Fernen unsre fern und ferner liegende Vergangenheit. In irgend einem Punkte des Raumes vergegenwärtigt jetzt erst das Licht die Scenen der französischen Revolution. In noch weiterer Ferne ist die Völkerwanderung erst jetzt an der Tagesordnung, zieht Alexander der Große noch siegend in der Welt umher. [...] In noch weitern Fernen des Raumes wird die Vergegenwärtigung der irdischen Vergangenheit durch das Licht erst in der Zukunft vor sich gehen, werden geschichtliche Ereignisse erst geboren werden, die für uns längst gestorben sind. ${ }^{9}$

Schon 1855, in der ersten Ausgabe der Volksbücher, hatte Bernstein im siebten Band die Einsichten des seiner Ansicht nach Herschel ebenbürtigen Anonymus ausführlich dargelegt und auch bibliographische Angaben beigefügt: Die Geschwindigkeit des Lichts, welches in so ungemein kurzer Zeit so ungeheure Räume durcheile, und der Gedanke, dass dieser Bote aus der Ferne oft Jahrtausende unterwegs sei und Nachrichten bringe aus Zeiten, welche längst geschwunden sind, gebe Anlass zu tiefgründigen Betrachtungen über Raum und Zeit.

Wir folgen bei diesen Betrachtungen einer kleinen Schrift, welche uns ein unbekannter scharfsinniger Denker vor mehreren Jahren in Breslau herausgab, einem Schriftchen, welches das Verdienst der Kürze und des Gedankenreichtums in so hohem Maße besitzt, daß wir nicht umhin können, zu wünschen, daß dessen Verfasser die Lesewelt mit ferneren Gaben erfreuen möge.

Als Fußnote war hinzugefügt: „Der Titel dieses Schriftchens ist: ,Der Mensch und die Sterne von X.Y.Z. Breslau 1846“" "10 Das war, halbwegs korrekt zitiert (es sei denn, es gab eine nicht mehr auffindbare Vorversion), die erste anonyme Ausgabe der Ebertyschen Schrift gewesen. Auch diesen hier nur anzitierten Text, der Ebertys Weltraumperspektiven - Vergangenheit als Lichtbildrealität zwischen den Sternen - mit bewegten Worten schilderte, hat Einstein in einem speziellen Kapitel Über die Geschwindigkeit des Lichts in seiner Bernstein-Ausgabe unverändert lesen können. ${ }^{11}$ Alles, was in den Zeiten der Vergangenheit liege, hatte Bernstein, Ebertys Ideen zusammenfassend, geschrieben, ,all' das existiert noch irgendwo, sobald man das Auge an die richtige Stelle bringt,

9 Bernstein (wie Anm. 8), S. 100-101.

10 Bernstein, Aaron: Aus dem Reiche der Naturwissenschaft. Für Jedermann aus dem Volke, Berlin 1855, Bd. 7, Kapitel 2 „Von der Geschwindigkeit des Lichtes “, S. 78-108, spez. S. 102.

11 Bernstein, Aaron: Naturwissenschaftliche Volksbücher, Wohlfeile Gesammt-Ausgabe, 4. Abdruck, 2. Bd., 8. Theil, Berlin o.J. [1873-74], S. 124159, spez. S. $152 \mathrm{ff}$. 
wo das Licht eben erst anlangt.“12 Bedarf es noch besonderer Betonung, dass solche Sentenzen die Phantasie des halbwüchsigen Einstein mächtig angeregt haben müssen?

\section{Felix Eberty}

Georg Friedrich Felix Eberty ${ }^{13}$ (1812-1884) stammte aus der wohlhabenden Berliner Ephraim-Familie ${ }^{14}$, die wie ein Buddenbrookscher Mikrokosmos Schicksal und Brillanz des Judentums in Deutschland verkörperte: aus ihren verschiedenen Zweigen sind unter anderen so bekannte Persönlichkeiten wie der Psychologe William Stern und der Kunsthistoriker Rudolf Wittkower hervorgegangen. Ebertys Urgroßvater, Nathan Veitel Chajim (Heine) ben Ephraim, war Hofjuwelier und wichtigster Münzunternehmer Friedrichs des Großen gewesen und hatte bedeutenden Anteil an der Finanzierung des siebenjährigen Krieges gehabt; sein Vater konvertierte zum christlichen Glauben und nahm den Taufnamen Hermann Julius Eberty an. Der Sohn Felix erhielt eine humanistisch-altsprachliche Schulausbildung und studierte Rechtswissenschaften, wie man in seinen Jugenderinnerungen eines alten Berliners nachlesen kann. Eberty trat als Kammergerichtsassessor in den Staatsdienst, wurde Richter und schließlich Stadtrat und außerordentlicher Professor für Natur- und Kriminalrecht an der Universität Breslau. Seine Jugendpassionen reichten jedoch viel weiter als sein späteres Berufsfeld und Lehrgebiet: Er war ein begabter Zeichner und betrieb nebenbei philosophische, literaturhistorische, mathematische und astronomische Studien.

Die erstmals 1846/47 in zwei Teilen unter dem Namenskürzel F.Y. erschienene Schrift über Die Gestirne und die Weltgeschichte war ein direkter Ausfluss derart vielseitiger Interessen. Eberty reagierte in den beiden praktisch anonym publizierten Heften gleichsam mit forensischem Scharfblick auf die jüngsten Leistungen der Astronomie: auf die ersten präzisen Parallaxenmessungen der nächstentfernten Fixsterne in den Jahren 1837-42. Noch knapp ein Jahrzehnt zuvor hatte John Herschel, berühmter Sohn des noch berühmteren Spiegelteleskop-Pioniers Sir William Herschel, mit resignierendem Tonfall den unauslotbaren Ab-

12 Bernstein (wie Anm. 10), S. 104; Bernstein (wie Anm. 8), S. 155.

13 Archiv Bibliographica Judaica. Lexikon deutsch-jüdischer Autoren, Bd. 6, München 1998, S. 40-43; Allgemeine Deutsche Biographie, Bd. 55, S. 473476; Neue Deutsche Biographie, Bd. 4, S. 546.

14 Michaelis, Dolf: „The Ephraim Family“, in: Leo Baeck Inst. Year Book XXI/1976, S. 201-228 und ders.: „The Ephraim Family (II)“, in: Leo Baeck Inst. Year Book XXIV/1979, S. 225-246. 
stand der Fixsterne beklagt: Zwischen den äußersten Planeten- und Kometenbahnen unseres Sonnensystems und dem nächsten Fixstern liege ein Abgrund, dessen Tiefe auch nur einigermaßen genau zu bestimmen astronomische Beobachtungen bisher nicht erlaubt hätten; es sei unmöglich, einen immerhin noch so großen Abstand zu nennen, den die wirkliche Entfernung des nächsten Fixsterns nicht noch weit überschreiten könnte. $^{15}$

Schon Tycho Brahe hatte Versuche unternommen, von der, Standlinie“ des Erdbahndurchmessers, d.h. von gegenüberliegenden Halbjahres-Bahnpunkten aus die kleine Verschiebung - die Parallaxe - mutmaßlich näher gelegener Sterne vor dem Himmelshintergrund zu messen; ohne Erfolg. Auch zwei Jahrhunderte später konnte der berühmte englische Astronom James Bradley trotz wesentlich verbesserter Instrumente und Beobachtungsmethoden noch keine Messergebnisse erzielen. Erst Friedrich Wilhelm Bessel (1784-1846), dem Hipparch des 19. Jahrhunderts, wie man ihn auch genannt hat, gelang es 1837-38 mit dem neuen Fraunhoferschen Heliometer an der Sternwarte Königsberg, für den Stern Nr. 61 im Sternbilde des Schwans verlässliche Messwerte zu ermitteln. In Dorpat, einem weiteren astronomischen Forschungszentrum in der baltischen Region, hatte gleichzeitig Friedrich Georg Wilhelm von Struve (1793-1864) erfolgreich Parallaxenmessungen durchgeführt. Die Parallaxenwinkel waren winzig, die Sternentfernungen, die sich ergaben, gewaltig, nur in Lichtlaufzeiten von Jahren statt in Meilen oder Kilometern halbwegs anschaulich anzugeben. Hinter den schwächsten, damals einzeln wahrnehmbaren Sternen, deren Entfernung auf bis zu viertausend Lichtjahre veranschlagt wurde, erstreckten sich weitere Weltenräume und Welteninseln, deren raumzeitliche Distanzen in Zehntausenden oder sogar Hunderttausenden von Lichtjahren zu schätzen seien, so Johann Heinrich von Mädler (1794-1874), Observator an der königlichen Sternwarte Berlin, später Direktor an der Sternwarte von Pulkowa/St. Petersburg und Autor zahlreicher populärwissenschaftlicher Darstellungen; und:

Wo keine unsrer Meßruthen den Raum, keine Geschichte die Zeit mehr zu umfassen vermag, wo unsre Erde nicht allein, sondern auch die Sonne, ja ihr ganzes System zum unscheinbaren, nichts bedeutenden Punkte zusammenschrumpft, da muß allerdings der Phantasie ein Spielraum gestattet werden. ${ }^{16}$

15 Herschel, John F. W.: A Treatise on Astronomy, London 1833, S. 376-377.

16 von Mädler, Johann Heinrich: Der Wunderbau des Weltalls, 1841, S. 473. 
Die ersten exakten Messungen von Fixsternabständen waren eine Sensation; eine Art Kolumbus-Effekt machte sich bemerkbar: Man hatte, wenn auch nur mit Messinstrumenten, den unauslotbaren Abgrund zwischen der Erde und den Sternen übersprungen, dem imaginären Fuß der Menschheit erste Landungspunkte eingerichtet. Aber die Anreisezeiten des Lichts führten nicht nur in Spielräume der Phantasie, sondern auch zur erschütternden Einsicht in die unabänderliche Vergangenheit des sichtbar Gegenwärtigen. Diesen, erstmals von Francis Bacon vermuteten, nun erhärteten Sachverhalt hat Alexander von Humboldt 1845 im ersten Band seines Kosmos prägnant hervorgehoben. Das Licht der nächstgelegenen Fixsterne $\alpha$ Centauri, 61 Schwan und $\alpha$ Leier benötige, wie man nun durch Bessel, MacLear und Struve wisse, 3, 91/4 oder 12 Jahre, um die Erde $\mathrm{zu}$ erreichen; und so fort. Der Anblick des gestirnten Himmels biete also nunmehr erwiesenermaßen Ungleichzeitiges dar; angesichts der [erstmals 1675 von Olaf Römer gemessenen] Lichtgeschwindigkeit sei es mehr als wahrscheinlich, dass das Licht der ferneren Weltkörper das älteste sinnliche Zeugnis vom Dasein der Materie überliefere. ${ }^{17}$

Dr. Eberty, nach 1840 am Amtsgericht Hirschberg und zeitweilig Richter in Lübben, bevor er um 1850 in Breslau seine Universitätskarriere ansteuerte, ging 1846 im ersten Heft seiner kleinen Schrift über Die Gestirne und die Weltgeschichte entscheidende Schritte über die ahnungsvollen Worte des Doyens der deutschen Naturwissenschaften hinaus. Er wandte das ihm juristisch vertraute Verfahren des parteiischen Blickpunktwechsels auf die Astronomie an und gelangte so $\mathrm{zu}$ dem ebenso plausiblen wie aufsehenerregenden Gedankenexperiment, fiktive Augenzeugen die Vergangenheit der Erde von unterschiedlich weit entfernten Fixsternen zu entsprechend verschiedenen Zeiten beobachten zu lassen. Wenn es möglich, d.h. den Gesetzen des Denkens nicht widersprechend sei, so Eberty, dass Menschen in beliebig kurzer Zeit zu anderen Sternen gelangen könnten, um von dort aus Fernrohre von gewaltiger Leistung auf die Erde zu richten, dann würde es im Prinzip auch möglich sein, ,jeden vergangenen Moment aus der Geschichte noch einmal sinnlich und im wahren und treuesten Bilde vor das sinnliche Auge zu rufen." So könne durch den Lauf der Jahrhunderte abwärts bis auf die neueste

17 von Humboldt, Alexander: Kosmos. Entwurf einer physischen Weltbeschreibung, Bd. 1, Stuttgart/Tübingen 1845, S. 159-161. 
Zeit jeder vergangene Augenblick wieder in die Gegenwart zurückberufen werden. ${ }^{18}$ Ebertys Fazit:

Wie ein ewig unverwüstliches und unbestechliches Archiv, dessen Inhalt lauterste, unmittelbarste Wahrheit ist, umschließt so der Weltenraum die Bilder des Vergangenen - Und wie der Schall Welle auf Welle in der Luft sich fortpflanzt [...] so pflanzen sich, unserer Betrachtung zufolge, von jeder Erscheinung die Luft- und Lichtbilder in den fernen Aether auf den Fittichen des Lichtstrahles fort. [...] Vorhanden ist also jenes in den Weltenräumen sich weiter und weiter auf den Schwingen des Lichts ausbreitende Archiv, wirklich und wahrhaftig, und mit Augen, wenn auch mit schärferen als menschlichen zu schauen. - Die Bilder aller geheimen Thaten, die geschahen, leben unauslöschlich und unvertilgbar von Ewigkeit zu Ewigkeit und von Sonnenferne zu Sonnenferne weiter. - Nicht nur auf den Dielen des Zimmers läßt die Mordthat ihre unauslöschlichen Blutspuren zurück, - auch in den Räumen des Himmels spiegelt die That sich weiter und weiter. ${ }^{19}$

Als Beispiele ungesühnter und doch beweiskräftig in himmlischen Lichtbildern dokumentierter Verbrechen hat Eberty die Verschleppung Caspar Hausers und den Mord an Karl XII. [von Schweden] angeführt.

\section{Charles Babbage}

Ebertys Lichtbildarchiv im Weltenraum war offensichtlich abgeleitet von jenem unzerstörbaren (Kriminal-)Archiv der von Schallwellen angeregten Atome, das Charles Babbage kurz zuvor - 1837/38 - in seinem berühmten Bridgewater-Buch dargelegt hatte:

The pulsations of the air, once set in motion by the human voice, cease not to exist with the sounds to which they gave rise. [...] The waves of air thus raised, perambulate the earth and ocean's surface, and in less than twenty hours every atom of its atmosphere takes up the altered movement due to that infinitesimal portion of the primitive motion which has been conveyed to it through countless channels, and which must continue to influence its path throughout its future existence. [...] Thus considered, what a strange chaos is this wide atmosphere we breathe! Every atom, impressed with good and with ill, retains at once the motions which philosophers and sages have imparted to it, mixed and combined in ten thousand ways with all that is worthless and base. The air itself is one vast

18 Eberty, Felix: Die Gestirne und die Weltgeschichte. Gedanken über Raum, Zeit und Ewigkeit, Breslau 1846, S. 19-20.

19 Eberty (wie Anm. 18). 
library, on whose pages are for ever written all that man has ever said or woman whispered. [...]

Auch den ethisch-kriminologischen Impetus hat Eberty von Babbage übernommen:

If the Almighty stamped on the brow of the earliest murderer - the indelible and visible mark of his guilt, - he has also established laws by which every succeeding criminal is not less irrevocably chained to the testimony of his crime; for every atom of his mortal frame, through whatever changes its severed particles may migrate, will still retain, adhering to it through every combination, some movement derived from that very muscular effort, by which the crime itself was perpetrated. [...] When man and all his race shall have disappeared from the face of our planet, ask every particle of air still floating over the unpeopled earth, and it will record the cruel mandate of the tyrant. Interrogate every wave which breaks unimpeded on ten thousand desolate shores, and it will give evidence of the last gurgle of the waters which closed over the head of his dying victim: confront the murderer with every corporeal atom of his immolated slave, and in its still quivering movements he will read the prophet's denunciation of the prophet king. ${ }^{20}$

Aber es gab auch bedeutsame Unterschiede zwischen den hypothetischen Kriminalarchiven Babbage's und Ebertys: Während der englische Mathematiker das Archivmaterial gewissermaßen aus den Opfern und Tätern selbst hervorgehen ließ und im Nahraum der Erdatmosphäre speicherte, sodass eigentlich sogar eine zeitinvertierte Rekonstitution der Akteure mit den Evidenzverfahren verbunden sein musste, sah der preuBische Jurist die Weltenräume von enteilenden immateriellen Lichtbilderstapeln erfüllt, die nur noch Einsicht und Überblick, aber keine Einmischung mehr erlaubten. Das betraf letztlich sogar den allerhöchsten Augenzeugen, den Eberty aufrufen konnte: Gott; - auch wenn dessen Eingriffsmöglichkeiten nicht eigens thematisiert waren.

Wir erhalten nämlich hier eine vollkommen faßliche und begreifliche Versinnlichung des Begriffes von der Allwissenheit Gottes in Bezug auf vergangene Dinge. Denken wir uns Gott in rein menschlicher Weise, mit menschlichen Kräften, nur in erhöhtem Maaße ausgestattet, so wird es uns ein leichtes sein, ihm die Fähigkeit und Macht zuzuerkennen, alles dasjenige, was sich von einem wirklichen örtlichen Standpunkte aus, sinnlich und leibhaftig überschauen und erblicken läßt, auch wirklich zu überschauen und bis

20 Babbage, Charles: The Ninth Bridgewater Treatise A Fragment [1837], Philadelphia 1841, Chapter IX. „On the Permanent Impression of our Words and Actions on the Globe we inhabit". 
ins Einzelnste zu unterscheiden. Wenn wir uns alsdann anschaulich machen wollen, wie vor Gott irgend eine vergangene irdische That oder Begebenheit, noch nach Jahrtausenden ebenso unmittelbar daliegt, als geschähe sie gegenwärtig unter seinen, so zu sagen leiblichen Augen, so genügt es zu diesem Zwecke, die Gottheit auf einem Punkte gegenwärtig zu denken, zu welchem gerade jetzt das Licht und die Abspiegelung jener Begebenheit gelangt. [...] wenn wir uns das Auge Gottes an jedem Punkte des Raumes anwesend denken, so gelangt $\mathrm{zu}$ ihm auch zugleich und auf Ein Mal der ganze Verlauf der Weltgeschichte. ${ }^{21}$

\section{Allgegenwart}

Die dem göttlichen Auge zugedachte Allgegenwart brachte Eberty auch zu bemerkenswerten darstellungstheoretischen Überlegungen:

Hier haben wir also die Ausdehnung der Zeit mit der des Raumes zusammenfallend, der sinnlichen Anschauung so nahe gebracht, daß Zeit und Raum als gar nicht von einander geschieden begriffen werden können. - Denn: das in der Zeit nacheinander Folgende liegt hier räumlich gleichzeitig neben einander. - Die Wirkung folgt nicht später auf die Ursache, sondern sie liegt räumlich und sichtbar neben ihr, und vor uns hat sich ein Gemälde ausgebreitet, welches Raum und Zeit zugleich umfaßt, und beide so im Ganzen und auf Einmal darstellt, daß wir räumliche und zeitliche Ausdehnung gar nicht mehr zu trennen und zu unterscheiden vermögen. $^{22}$

Bedenkt man, mit welcher Hartnäckigkeit die etablierte Ästhetik zur gleichen Zeit noch die Lessingschen Kategorien und von seinen Anhängern verschärften Normvorschriften des entweder bildlich simultan koexistierend oder sprachlich konsekutiv zu Entfaltenden propagierte, so kommt einem die Kühnheit der Ebertyschen Überlegungen erst so recht zum Bewusstsein. Es hat noch ein halbes Jahrhundert gedauert, bis der Wiener Kunsthistoriker Franz Wickhoff in seiner Untersuchung der Wiener Genesis das Paradigma der Sukzession in die Welt des sichtbar Dargestellten übertrug und den Begriff der kontinuierenden Bilderzählform prägte. „Diese Art des Erzählens ist sehr auffällig“, hat Wickhoff $1895 \mathrm{zu}$ Beginn seines Faksimile-Kommentars geschrieben.

Sie weicht auch von der, die wir in der Kunst unserer Tage zu sehen gewohnt sind, vollständig ab. Hier wird nicht ein entscheidender Moment gewählt, der die wichtigsten Personen des Textes

21 Eberty (wie Anm. 18), S. 14-15.

22 Eberty (wie Anm. 18), S. 15-16. 
$\mathrm{zu}$ einer gemeinsamen folgenreichen Handlung vereinigt, um sie uns in einem zweiten Bilde in anderer, nicht minder bedeutender Situation zu zeigen, während in einem dritten und vierten wieder mit Überlegung ausgewählte Szenen die Erzählung fortsetzen. Nicht einzelne Bilder ausgezeichneter, epochemachender Augenblicke treten $\mathrm{zu}$ einem Zyklus zusammen [...], sondern, wie der Text strömt, begleiten ihn, sanft gleitend und ununterbrochen, gleichwie die Uferlandschaften bei einer Wasserfahrt an dem Auge vorüberziehen, die jeweiligen Helden der Erzählung in kontinuierlich sich aneinanderreihenden Zuständen. ${ }^{23}$

Wickhoff hatte nur den Erzählstil einer bestimmten Kunstepoche, den der reichsrömischen Spätantike, im Sinn. Eberty war es um wahrhaft universelle Eigenschaften hypothetischer Lichtbilder und entsprechender Wahrnehmungsweisen gegangen. Gleichwohl ist die Übereinstimmung der Betrachterprospekte - dort interstellare Lichtbilderströme, hier vorübergleitende Uferlandschaften - verblüffend.

\section{Gedankenexperimente}

Eberty hat nicht nur eine imaginäre Retrospektivoptik der multiplen, aber fixierten Sternenstandpunkte entworfen, die den sensationellen neuen Entfernungsmessungen entsprach, sondern das fiktive Inspektionssystem auch durchgängig dynamisiert:

Denken wir uns einen mit unendlich erweiterter Sehkraft begabten Beschauer auf einem Sterne 12. Größe, von wo aus er in diesem Augenblicke also die Erde in dem Zustande erblicken würde, wie sie zu den Zeiten Abrahams beschaffen war. - Denken wir uns ferner diesen Beschauer mit einer solchen Schnelligkeit in der Richtung nach unserer Erde fortbewegt, daß er in einer kurzen Zeit, nehmen wir an in einer Stunde, bis auf die Entfernung von 20 Millionen Meilen von unserer Erde gelangt, wo er uns so nahe wäre, wie uns die Sonne jetzt ist, und wo also die Erde ihm in diesem Augenblicke so erscheinen müßte, wie sie vor acht Minuten gewesen, - denken wir uns alles dieses, ganz abgesehen von allen Ansprüchen an Möglichkeit und Wirklichkeit, - so ergiebt sich doch unwidersprechlich folgendes: daß vor dem Auges dieses Beschauers die ganze Weltgeschichte, von den Zeiten Abrahams bis auf diesen heutigen Tag, in Zeit von einer Stunde vorübergeführt worden ist. [...] Es bedarf keines weiteren Beweises, sondern es leuchtet von selbst und ohne möglichen Widerspruch ein, daß, wenn ein Auge im Stande wäre, den wirblenden Zug der auf einander folgenden Bilder zu fassen, dieser Beschauer nothwendig die ganze Weltgeschichte, mit allen den Handlungen und Begebenhei-

23 Wickhoff, Franz: Römische Kunst [1895], Berlin 1912, S. 9-10. 
ten, die auf der ihm jedes Mal zugekehrten Erdhalbkugel seit 4000

Jahren vorgefallen sind, in einer Stunde durchlebt haben müßte. ${ }^{24}$

An dieser Beschreibung der auf eine Stunde zusammengedrängten Weltgeschichte von 4000 Jahren springt zuletzt das ,kleine' technische Detail der rotierenden Erdkugel ins Auge, die dem heranrasenden Betrachter jedes Mal dieselbe Seite zukehren muss, um eine orts- und sogar personenbezogene Kontinuität der Bilderfolgen zu gewährleisten. Erkennbar hat hier das Prinzip der stroboskopischen Scheiben und Zylinder, das 1832 der Mathematiker Simon von Stampfer in Wien und der belgische Physiker Joseph Anton Ferdinand Plateau entdeckten, Pate gestanden. Die entsprechenden Geräte wurden ihrerzeit Phänakistoskop oder Phantaskop, also ,Täuschungsseher' genannt, um den Illusionscharakter der wahrgenommenen Scheinbewegungen zu kennzeichnen. Machte Ebertys Phantasie also aus der Erde ein Riesengerät zur stroboskopischen Betrachtung von globalen Bildergeschichten? - Die Schilderung bezog sich sehr wohl auf derartigen Illusionscharakter, behauptete unmittelbar anschließend aber gleichwohl die Lückenlosigkeit der Bildfolgen: ,Alles ganz und unverkürzt, nur in der schnellsten Aufeinanderfolge." Ein latenter Widerspruch zwischen implizitem Vorbild und seiner Fiktionalisierung also, der sich in weiteren Besonderheiten artikulierte:

Geben wir diesem, den Aether durchfliegenden Beschauer noch die Möglichkeit, auf seiner Bahn beliebig anzuhalten, so wird er jeden Moment der Weltgeschichte, den er mit Muße vor sich abspielen lassen will, dadurch vollständig und in beliebiger Schnelligkeit vor seinen Augen aufführen lassen können, daß er, so lange es ihm gefällt, in einer solchen Entfernung verweilt, wo dieser vergangene Moment der Geschichte im Verhältnis zur Zeit, die das Licht braucht, um bis zu dem Standpunkte des Beschauers zu dringen, gerade gegenwärtig erscheint. ${ }^{25}$

Solche Vorstellungen vom vollständigen Innehaltenkönnen neben oder vor (?) den mit Lichtgeschwindigkeit bewegten Bildern entsprach der schnelleren oder langsameren Rotation der stroboskopischen Scheiben und Zylinder; bis hin zum vollständigen Stillstand, der allerdings realiter mit dem Zusammenbruch der Bewegungsillusion einherging. Auch wenn Eberty einen solchen Aggregatwechsel der Wahrnehmung nicht in Betracht zog: - Hatte man es hier nicht eindeutig mit einer von der Experimentaltechnologie abgeleiteten, sekundären Fiktion zu tun?

24 Eberty (wie Anm. 18), S. 23-25.

25 Eberty (wie Anm. 24). 
So direkt ein Zusammenhang erkennbar ist, so deutlich sind auch die Unterschiede: Die Lichtbilder waren nicht mehr materiell an den Bildträger gefesselt gedacht; und der fiktional neben ihnen bewegte Bilderbetrachter hatte den eigentlich aktiven Part der Wahrnehmung übernommen. Nicht die Rotationsmechanik der Stroboskop-Scheiben und -Zylinder bestimmte den Lauf oder Stillstand der Bilder, sondern die Volition, das willentliche Verweilen oder Davoneilen des imaginierten Augenzeugen. Ebertys Phantasien waren nicht zweitrangige Adaptionen einer bereits existierenden Illusionstechnik, sondern ein großangelegtes Gedankenexperiment - ein Begriff, der im selben Jahrzehnt von Hans Christian Oersted geprägt wurde. ${ }^{26}$ Ebertys Fiktion bediente sich einzelner Elemente der instrumentell erzeugten Realerfahrung, um sie auf neuartige Weise $\mathrm{zu}$ inszenieren und $\mathrm{zu}$ interpretieren. Die naturwissenschaftliche Stichhaltigkeit muss nach heutigen Maßstäben zweifelhaft erscheinen; aber die ideengeschichtliche Tragweite war außerordentlich.

\section{Mikroskop für die Zeit}

Im zweiten ,Heft' mit dem gleichen Titel, das wiederum unter dem Namenskürzel F.Y. 1847 publiziert wurde, hat Eberty seine Idee von der lichtbilderbegleitenden, aber eigenbeweglichen interstellaren Augenzeugenschaft noch anschaulicher und begrifflich prägnanter ausgesponnen:

In ganz gleicher Weise, wie durch eine unendlich beschleunigte Bewegung von einem Fixsterne nach der Erde zu, sich die Bilder der Weltbegebenheiten in einem Augenblick zusammendrängen lassen, ebenso läßt sich, umgekehrt, die Reihenfolge dieser Bilder ins Unendliche ausdehnen, und zwar in folgender Weise: - Nehmen wir an, daß das Licht, und mit ihm die Abspiegelung einer irdischen Begebenheit zu irgend einem Fixsterne zweiter Größe genau in zwanzig Jahren gelangt. Nehmen wir ferner an, daß der Beschauer in dem Augenblicke wo z.B. eine Blüthenknospe sich zu erschließen beginnt, bis zu diesem Fixsterne in einem Zeitraume von zwanzig Jahren und einem Tage aufsteigt, so wird er dort das Bild dieser Blume in demjenigen Stadium der Entwicklung antreffen, in welchem sie sich Einen Tag nach ihrem ersten Aufblühen befindet. - Wenn er nun, mit unendlicher Sehkraft und Beobachtungsgabe ausgerüstet, diese Blüthenentwicklung während der ganzen Reise verfolgen könnte, so würde er zwanzig Jahre lang Zeit gehabt haben, um diejenigen Veränderungen $\mathrm{zu}$ studiren, welche mit der Blume auf Erden während eines Einzigen Tages

26 Oersted, Hans-Christian: „Der allgemeinen Naturlehre Geist und Wesen“, in: ders.: Die Naturwissenschaft und die Geistesbildung, Leipzig 1851, S. 18 . 
vorgingen. - Wie man einen vorübergaukelnden Schmetterling kaum ins Auge fassen, und die Färbung seiner Flügel zu unterscheiden vermag, dagegen, wenn man ihm auf seinem Fluge betrachtend folgen könnte, gar wohl im Stande wäre, die Körnchen seines Farbenstaubes zu zählen und zu zergliedern, - ganz so würde der Beobachter, welcher der Abspiegelung einer flüchtigen Begebenheit auf den Fittichen des Lichtes zu folgen vermöchte, die schnellsten Entwicklungen mit größter Genauigkeit und Muße zergliedern können. - Es wäre auf diese Weise gewissermaßen ein Mikroskop für die Zeit gegeben. ${ }^{27}$

Was späterer Sprachgebrauch mit dem filmtechnischen Begriff der Zeitlupe belegte, war bei Eberty noch einem der maßgeblichen optischen Instrumente der Neuzeit, dem Mikroskop, zugeordnet. Aber das Mikroskop sollte sich auf Lichtbilder im Weltall richten, also in die Domäne des anderen Leitinstruments, des Teleskops, eindringen. Ein auf den ersten Blick irritierender Austausch. Die übliche Einteilung der instrumentellen Aufgabenbereiche hatte zum Beispiel der Koblenzer Naturkundelehrer des ,großen Physiologen“ Johannes Müller, Joseph Görres, noch 1802 mit folgenden Aphorismen umrissen:

Das Attribut des Idealisten ist das Teleskop, mit ihm dringt er in die Unendlichkeit hinaus, zu Lichtbündeln verlängern sich seine Sehnervenbündel, und mit diesen zarten Fühlfäden, die im Auge zusammenlaufen und von dort aus den ganzen Raum durchweben, betastet er die entlegensten Welten, als ob er sie in Händen trüge [...] Das Attribut des Realisten ist das Mikroskop, [...] er taucht unter in dem Wassertropfen und belauscht seine regen Bewohner; die ganze Natur außer seinem Gesichtsfelde ist ihm untergegangen, eine neue geht ihm dafür innerhalb desselben auf, und auch diese sucht er wieder zur Aeußern zu machen [...]. ${ }^{28}$

\section{Carl Gustav Carus}

Auf die Idee, die Zuständigkeitsbereiche von Mikroskop und Teleskop zu vermengen und sogar zu verschmelzen, ist wohl als erster Carl Gustav Carus gekommen. Im ersten seiner Briefe über das Erdleben hat er 1841 eine bemerkenswerte Mikroskop-Phantasie beschrieben: Er habe des Öfteren vorgehabt, einen Traum zu dichten, in dem einem starren Verfechter der Trennung von belebter und unbelebter Natur ,vom Weltgeiste

27 Eberty, Felix: Die Gestirne und die Weltgeschichte. Gedanken über Raum, Zeit und Ewigkeit, 2. Heft, Breslau 1847, S. 2-3.

28 Görres, Joseph: „Aphorismen über die Kunst“ [1802], in: Gesammelte Schriften, Band 2,1, Köln 1932, S. 148. 
die Anweisung kommt, durch ein im weiten Äther schwebendes Mikroskop zu blicken. Da sieht er denn bald leuchtende, bald erleuchtete Kügelchen in regelmäßigen Rotationen einander umkreisen, [...] er sieht, wie sie aufeinander anziehend wirken, wie hier und da ein Kügelchen zerfällt und verstäubt, während an andern Stellen aus nebelhaften Stoffen neue Pünktchen entstehen und nach weitern exzentrischen Umherrollen sich zu den gemessenen Kreisen der andern gesellen, sich dort durch Einsaugung nähren und vergrößern und durch Ausscheidung wieder verringern usw., und alles ruft ihm seine frühern mikroskopischen Beobachtungen über die leuchtenden See-Infusorien und über die ohne Mund und Eingeweide regelmäßig umherrollenden Kugeltiere so bestimmt zurück, dass er sich schon bereit macht, diese Äther-Infusorien als neue und besondre Sippe in sein System einzutragen. Aber da ertönen ihm nicht ohne Beschämung die seltsamen Worte: ,Was du gesehen, war die Bewegung von Sonnensystemen, und während du eine Stunde zu beobachten glaubtest, ist ein Weltenjahr vorübergegangen! “'29

Raffungseffekte der instrumentierten Wahrnehmung hätte Eberty also aus Carus' Schrift übernehmen können. Wenn dem so war, so hätte ihn der totale Täuschungscharakter des Vorbilds bei der Gleichsetzung von Mikro- und Makrokosmos auch zu seinen prinzipiellen Spekulationen über Zeiterfahrung veranlassen können: Denke man sich, dass der Gang der Gestirne und unserer Erde aufs doppelte beschleunigt würde, schrieb Eberty 1847, so dass auch alle menschlichen Lebensprozesse sich auf die Hälfte der Zeitdauer reduzierten; „-- unsere Athemzüge, unsere Pulsschläge würden gleichfalls in doppelter Schnelligkeit auf einander folgen, und dieses neue Lebenstempo nunmehr als das normalmäßig gesunde erscheinen." Würden wir solche Beschleunigung überhaupt bemerken? Ebertys Antwort: Wir würden keine Veränderung gewahr werden, weil wir den Verlauf der Zeit nur durch Vergleich mit anderen Zeitverläufen bestimmen könnten. Unsere vierzig Jahre würden uns wie achtzig erscheinen. Aber das war noch keineswegs das letzte Wort: Ganz dasselbe ergebe sich, so Eberty, wenn man den Lauf der Zeit, statt ums Doppelte, nunmehr auf das Vierfache beschleunigt denke; und weiter:

Ein gleiches Resultat wie bei einer vierfachen, ergiebt sich aber aus denselben Gründen auch bei einer tausendfachen, ja bei einer Millionenfachen, - mit einem Worte, bei einer unendlich beschleunigten Bewegung unseres Lebensprocesses, und des Ganges der Weltordnung um uns her, und wir könnten uns auf diese Weise den

29 Carus, Carl Gustav: Zwölf Briefe über das Erdleben, hrsg. v. Christoph Bernoulli/Hans Kern, Celle 1926, S. 19-20. 
ganzen Verlauf der Weltgeschichte in einen Einzigen unermessbar kurzen Zeitmoment zusammengedrängt denken [...]. ${ }^{30}$

\section{Karl Ernst von Baer}

Mit beschleunigten oder verzögerten Zeitläufen stellte sich also die Frage nach absoluten Maßstäben des subjektiven Erlebens. Eberty argumentierte, dass ein verdoppeltes, halbiertes, tausend- und millionenfach schnelleres oder langsameres Lebenstempo in entsprechend bewegten Umwelten gar nicht bemerkt werden würde, dass also Zeit, ebenso wie der Raum, letztendlich nur eine menschliche Auffassungsform sei. Dieses Konzept wurde - um eine der bemerkenswertesten Propagationslinien zu erwähnen - dreizehn Jahre später von dem berühmten St. Petersburger Biologen Karl Ernst von Baer aufgegriffen und zum Gedankenspiel von jeweils tausendfach beschleunigten 29-Tage- und 40-Minuten-Menschen oder entsprechend verzögerten Jahrtausend- und Jahrmillionenmenschen entwickelt, deren radikal veränderte Wahrnehmung ihrer nun allerdings nicht mitbeschleunigten oder mitverlangsamten Umwelten er eindrucksvoll beschrieben hat.

„Ueberhaupt scheint der Puls in gewisser Beziehung mit der Schnelligkeit von Empfindung und Bewegung zu stehen." Mit dieser Mutmaßung setzte von Baer - in einem Vortrag mit dem Titel Welche Auffassung der lebenden Natur ist die Richtige? - 1860 zur Explikation seiner dann häufig zitierten Überlegungen an;

Beim Kaninchen folgen sich die Pulsschläge 2 Mal so schnell als beim Menschen und beim Rinde fast 2 Mal so langsam. Sicher erfolgen Empfinden und Bewegung bei jenen Thieren auch viel schneller als bei diesen. Es erleben also die Kaninchen in derselben Zeit bedeutend mehr als die Rinder.

Mit diesen Mutmaßungen wolle er darauf aufmerksam machen, so Baer, dass das innere Leben in derselben äußeren Zeit bei Menschen und verschiedenen Tieren erheblich rascher oder langsamer verlaufen könne und dass dementsprechend auch die Wahrnehmung ihrer Umwelt - diesen Uexküllschen Schlüsselbegriff hat von Baer noch nicht benutzt, aber gemeint - sich abweichend gestalte. ${ }^{31}$

30 Eberty (wie Anm. 27), S. 15-16.

31 von Baer, Karl Ernst von: „Welche Auffassung der lebenden Natur ist die richtige? Und wie ist diese Auffassung auf die Entomologie anzuwenden?“ zur Eröffnung der Russischen entomologischen Gesellschaft im Oktober 
Angenommen, der Lebenslauf des Menschen verliefe viel rascher, als er wirklich verläuft, so von Baer, es würden ihm alle Naturverhältnisse ganz anders erscheinen. Denken wir uns einmal, sein Leben wäre auf den tausendsten Teil beschränkt. Er wäre schon sehr hinfällig, wenn er 29 Tage alt ist, gleichwohl aber fähig, im Zeitraum eines Pulsschlages sechs bis zehn sinnliche Wahrnehmungen aufzufassen. Er würde gar Manches sehen, was wir nicht zu sehen vermögen, zum Beispiel eine vorbeifliegende Flintenkugel; und so fort:

Denken wir uns aber das menschliche Leben noch sehr viel mehr verkürzt, und zwar gleich auf den tausendsten Theil des schon oben verkürzten Maaßes, so würde seine Dauer nur 40, und wenn es hoch kommt 42 Minuten ausfüllen. Bliebe die übrige Natur dabei völlig unverändert, sie würde uns doch wieder ganz anders erscheinen. [...] Alle Töne, welche wir hören, würden freilich für solche Menschen unhörbar sein, wenn ihr Ohr ähnlich organisiert bliebe als das unserige, dagegen würden sie vielleicht Töne vernehmen, die wir nicht hören, ja vielleicht würden sie sogar das Licht, welches wir sehen, nur hören. ${ }^{32}$

Wie er das menschliche Leben im Verhältnis zur Außenwelt verkürzt und gleichsam in sich verdichtet vorgestellt habe, so von Baer, so ließe es sich auch tausend mal verlangsamt denken. Die Sonne würde dann wohl, bei der scheinbaren Schnelligkeit ihrer Bewegung, einen feurigen Schweif hinterlassen; und so weiter.

Wenn wir das tausendfach verlangsamte Menschenleben noch auf das Tausendfache langsamer annehmen, so würde ihm die äußere Natur wieder ganz anders sich zeigen. [...] Wir könnten den regelmäßigen Wechsel von Tag und Nacht nicht erkennen. Ja, wir würden die Sonne nicht einmal erkennen, sondern, wie eine rasch im Kreise geschwungene glühende Kohle als leuchtender Kreis erscheint, würden wir den Sonnenlauf nur als leuchtenden Bogen am Himmel sehen. ${ }^{33}$

Der Mensch vermöge nur mit sich selbst - anhand seiner sinnlichen Organisation - die Natur sowohl räumlich als auch zeitlich zu messen, so von Baers Fazit, „weil es ein absolutes Maaß nicht giebt.“ Welche Ansicht der Natur sei also die der Wahrheit am nächsten kommende? „Ohne Zweifel die, welche aus dem größeren [mehr Zeit umfassenden] Maaßstabe hervorgeht. [...] Der Maßßstab für ihre Wirksamkeit kann nie

1860 gesprochen, in: Reden gehalten in wissenschaftichen Versammlungen, Braunschweig 1886, S. 258.

32 von Baer (wie Anm. 31), S. 260-262.

33 von Baer (wie Anm. 31), S. 265-266. 
zu groß sein, sondern ist immer zu klein.“34 - Welches war aber der denkbar größte Maßstab? Ebertys Weltgeschichte in einem einzigen Augenblick? Zu solchen Extremen hat von Baer nicht Stellung genommen; so, wie er auch keine Inspirationsquellen seiner Darlegungen genannt hat.

Dass von Baer seine Überlegungen zur Naturauffassung ganz ohne Kenntnis der Ebertyschen Schrift entwickelt haben sollte, scheint mir auf Grund der akkuraten Übereinstimmung der genannten Beschleunigungsbeziehungsweise Verzögerungsfaktoren - zwei, vier, tausend, eine Million - extrem unwahrscheinlich. Im Gegenteil, gerade die Erhebung der subjektiven Zeitwahrnehmung zum Maßstab macht die sinnesphysiologische Antithese zum rein spekulativen Lichtbilderspektakel Ebertys erst vollends verständlich. Und als um so bedeutsamer beginnt sich auch die Rolle der kleinen anonymen Schrift des Berliner Juristen abzuzeichnen, von der eine wahre Lawine von wissenschaftlichen Spekulationen und eigentümlichen Weltbetrachtungsweisen ausgelöst worden ist.

\section{Christian Doppler}

Ebertys hypothetische Lichtbilderinspektoren hätten bei ihren rasanten Ortswechseln im Weltenraum eigentlich ähnlich radikalen Verschiebungen der Sinneseindrücke ausgesetzt sein müssen wie von Baers beschleunigte oder verlangsamte 40 Minuten- oder Jahrmillionenmenschen. Und noch fundamentalere physikalische Einschränkungen kamen hinzu: Bereits 1842 hatte der österreichische Physiker Christian Doppler (18031853) erstmals mit dem nach ihm benannten Effekt die Variabilität von Sinneseindrücken in Abhängigkeit von Bewegungen dargelegt. - Aber nicht der heutigentages mit seinem Namen verbundene akustische Doppler-Effekt war sein Ausgangspunkt gewesen, sondern das farbige Licht der Doppelsterne. ${ }^{35}$

Nach der ursprünglichen Vibrationshypothese sei bekanntlich die Farbempfindung eine unmittelbare Folge der in gewissen Zeitintervallen regelmäßig aufeinanderfolgenden Wellenschläge des Äthers, so Doppler.

34 von Baer (wie Anm. 31), S. 267-268.

35 Doppler, Christian: „Ueber das farbige Licht der Doppelsterne und einiger anderer Gestirne des Himmels. Versuch einer das Bradley'sche Aberrationstheorem als integrirenden Theil in sich schliessenden allgemeineren Theorie", in: Abhandlungen der Böhmischen Gesellschaft der Wissenschaften, V. Folge [1842], Bd. 2; reproduziert in: Doppler, Christian: „Schriften aus der Frühzeit der Astrophysik“, in: Ostwalds Klassiker der exakten Wissenschaften, Bd. 161, Frankfurt a.M. 2002, S. 5-7. 
Die Intensität des farbigen Lichtes hänge lediglich von der Größe der Exkursionen [Schwingungsbewegungen] der einzelnen Ätherteilchen ab, welche die Netzhaut des Auges berühren. Alles, was demnach das Zeitintervall zwischen den einzelnen Ätherstößen verändere, ziehe notwendig eine Änderung der Farbe nach sich, während die Intensität des Lichts von der Energie der Wellenschläge abhänge. Aber man habe bisher völlig vernachlässigt, danach zu fragen, wie die Äther- oder auch Luftschwingungen von den Sinnesorganen aufgenommen und empfunden werden. Solange man voraussetze, dass sowohl der Beobachter als auch die Wellenquelle ihre anfänglichen Orte beibehalten, würden die subjektiven Bestimmungen mit den objektiven vollkommen übereinstimmen. Wie aber, wenn entweder der Beobachter oder die Quelle oder gar beide ihren Ort veränderten, sich voneinander entfernten oder einander näherten; und dieses mit Geschwindigkeiten, welche an die der Wellenfortpflanzung heranreichten? - Dopplers zunächst anschaulich, dann mathematisch gefasste Antwort: Wie ein Schiff, welches den andringenden Wellen gerade entgegensteuere, in derselben Zeit eine größere Anzahl und viel heftigere Wasserwellenschläge erleide, so sei auch mit einer Häufung und Steigerung der Äther- oder Luftwellen bei gegenläufiger Bewegung zu rechnen; und so weiter. Solche bildhaft untermauerten Vorüberlegungen brachten Doppler zu der Vermutung, die Lichtwellen von Doppelsternen, die einander rasch umkreisen, sowie von so genannten periodisch Veränderlichen könnten so stark gerafft oder gedehnt sein, dass sie für menschliche Augen nicht nur farblich weitgehend verändert, sondern unsichtbar würden; - denn sogar Sternbewegungen mit Überlichtgeschwindigkeit hielt Doppler für möglich.

Seit der Entdeckung der Infrarot- und Ultraviolettstrahlung 1800/01 hatte man sich zunehmend bewusst gemacht, dass der Mensch mit seinen Sinnesorganen nur schmale Fenster in die Welt der mechanischen, Schall-, Wärme- und Lichtschwingungen öffnen kann. Dass Eberty den sternenbezogenen Doppler-Effekt, der für seine Betrachtungen doch von grundlegender Relevanz hätte sein müssen, nicht in seine Spekulationen mit einbezogen hat, könnte auf den entlegenen Publikationsort zurückzuführen sein. Dopplers erster Aufsatz wurde in den Abhandlungen der Böhmischen Gesellschaft der Wissenschaften gedruckt. Aber es hatte kritische Rezensionen, z.B. des hochrenommierten Berliner Hofraths und Dorpater Sternwartendirektors Mädler, und empfindliche Repliken Dopplers gegeben, von denen Eberty gehört haben könnte. Gleichwohl hat Eberty auch später die Auswirkungen des Doppler-Effekts auf seine rasch bewegten Weltraumbeobachter nicht in Betracht gezogen. Hätte 
deren Wahrnehmung nicht wegen der extrem gequetschten oder zerdehnten Ätherwellen versagen müssen? Wie mochten Lichtbilder überhaupt aussehen, wenn man sich neben ihnen mit Lichtgeschwindigkeit bewegte? Für prinzipielle Gedankenexperimente, die in höchster Instanz den Allmächtigen selbst als Bilderinspektor bemühten, mögen solche Fragen eher zweitrangig erschienen sein. - Wie immer es sich verhalten haben mag: Vorerst bleibt nur festzuhalten, dass Ebertys astronomische Phantasien schon zum Zeitpunkt ihrer Publikation physikalisch in Frage gestellt waren. Aber das hat ihrer ungeheuren Wirkungsgeschichte keinen Abbruch getan.

\section{H. Bailliere, Publisher}

Die ersten publizistischen Reaktionen auf Ebertys neuartige Bildwissenschaft hatten schon unmittelbar nach Erscheinen des ersten Heftes eingesetzt. Der Londoner Verlag Bailliere brachte noch im Erscheinungsjahr 1846 eine anonyme Übersetzung unter dem Titel The Stars and the Earth;Thoughts upon Space, Time and Eternity heraus. 1847 folgte sofort eine definitiv nicht autorisierte Übersetzung des zweiten Teils, wie aus einer Drucknotiz im deutschen Original hervorgeht, derzufolge Eberty offenbar einen Lizenzvertrag mit Bailliere geschlossen, sich aber das Recht der eigenen Übersetzung ins Englische vorbehalten hatte. ${ }^{36}$ Wie immer es sich im Einzelnen abgespielt haben mag, Bailliere setzte auch den zweiten Teil ohne jede Namensnennung in Umlauf, der bald in Eins verlegte Zweiteiler wurde ein Riesenerfolg, in England und Amerika wurden viele Nachdrucke und Neuauflagen in zehntausenden von Exemplaren verkauft; die Gesamtzahl wird in der Größenordnung von hunderttausend gelegen haben.

„Fourty-eight small pages, suggesting food for a life of thought", lautete die Quintessenz einer der zahlreichen lobenden Pressestimmen, die schon bei Erscheinen des zweiten Teils 1847 mitzitiert wurden. Die zweite vollständige amerikanische Ausgabe von $1850^{37}$, der dritten englischen nachgedruckt, warb mit einem in den Reprints immer wieder beigefügten Empfehlungsschreiben eines gewissen Reverend Thomas Hill - der war nebenbei ein exzellenter Astronom, später Präsident von

36 Die Drucknotiz lautet: Der Verfasser behält sich das Recht vor, eine englische Uebersetzung zu liefern, und hat das, nach dem Vertrage vom 13. Mai 1846 verlangte Exemplar in London deponiert.

37 Eberty, Felix: The Stars and the Earth; Thoughts upon Space, Time and Eternity, Boston 1850. 
Harvard - vom 24. Juli 1849, in dem bereits der Bezug zu Babbage's Bridgewater-Treatise und dessen atmosphärischem Kriminalarchiv hergestellt wurde. Man sah das erstaunliche anonyme Büchlein ganz in der Traditionslinie angloamerikanischer Wissenschaftstheologie. Von diesem namenlosen Bestseller-Erfolg im Ausland hat sein deutscher Autor wenig profitiert. Nicht einmal sein Name, dessen Anfangs- und Endbuchstabe FY immerhin die Originalversion geziert hatten, fand Eingang in die englischsprachige Gelehrtenwelt, als es Eberty schließlich 1874 gelang, die deutschen Verlagsrechte zurückzugewinnen und eine leicht überarbeitete Neuausgabe unter seinem vollen Namen herauszubringen. ${ }^{38}$

\section{Camille Flammarion}

Ruhm und Profit der Ebertyschen Ideen waren einem Anderen zugefallen, der ein begnadeter Requisiteur und Popularisator war. Camille Flammarion veröffentlichte im Jahre 1867 Lumen, récit d'outre terre ${ }^{39}$, die erste von vier Erzählungen, die von 1872 an unter dem Titel Récits de l'infini mit zwei anderen Texten als Buch zusammengefasst und 1887 in erweiterter, dann auch illustrierter Fassung unter dem Titel Lumen $^{40}$ große Auflagenzahlen erreichten. In diesen récits steht ein Verstorbener, LUMEN genannt, einem neugierigen Lebenden - QUÆRENS, dem ,Fragenden " - Rede und Antwort. Nach seinem Tode im Jahr 1864 habe der, LUMEN, sich auf einen Planeten des Sterns Capella versetzt gefunden und von dort aus dank seines nun überirdisch scharfen Sehvermögens die Erde zu Zeiten seiner Geburt erblickt und Szenen seiner eigenen Jugend; und so weiter. In langatmig umständlicher und gleichwohl spannender Erzählung sind alle wesentlichen Momente der Ebertyschen Fiktionen aufgenommen und weiterentwickelt. Verteilt auf die vier zwischen 1865 und 1869 geschriebenen Geschichten finden sich zentrale Gesichtspunkte der Ebertyschen Lichtbildergeschichte fast wortwörtlich wiederholt; allem voran die Allgegenwärtigkeit der Weltgeschichte für den Schöpfer.

38 Eberty, Felix: Die Gestirne und die Weltgeschichte. Gedanken über Zeit, Raum und Ewigkeit, Breslau 1874.

39 Flammarion, Camille: „Lumen, récit d'outre-terre“, in: Revue du xix siècle, 11 (1867), S. 163-184. Die Fortsetzungen erschienen in L'Artiste, revue dirigée par Arsène Houssaye.

40 Flammarion, Camille: Lumen. Illustrations de Lucien Rudaux, Paris o.J. [1887]. 
Les événements qui se sont accomplis à la surface de la Terre, depuis son origine, sont visibles dans l'espace à des distances d'autant plus éloignées qu'ils sont plus reculés. Toute l'histoire de la Terre, et la vie de chacun de ses habitants pourraient donc etre vues à la fois par un regard qui embrasserait tout cet espace. Nous comprenons optiquement par là que Dieu, présent partout, voie tout le passé dans un meme moment. Ce qui est vrai de notre Terre est vrai de tous les mondes de l'espace. Ainsi l'histoire entière de tous les univers peut être présente à la fois dans l'universelle ubiquité du Créateur. ${ }^{41}$

Was Eberty mit juristisch vorsichtiger Sprachpräzision als Lichtbilder ${ }^{42}$ bezeichnete, hat Flammarion griffiger, aber auch leichter missverständlich mit Photographien gleichgesetzt:

Voilà, dis-je, une sérié de photographies terrestres échelonnées dans l'espace à ces intervalles réciproques. Supposons maintenant qu'entre chacune de ces images séculaires se trouvent échelonnées à leur tour les images annuelles, gardant entre chacune d'elles la distance que la lumière parcourt en un an, et que je viens de vous nommer; puis qu'entre chacune des images annuelles, nous ayons les images de chaque jour; puis que chaque jour contienne les images de ses heures, chaque heure enfin les images de ses minutes et chaque minute les images de ses secondes, le tout se succédant suivant les distances respectives de chacune d'elles: nous aurons dans un rayon de lumière, ou pour mieux dire dans un jet de lumière composé d'une série d'images distinctes juxtaposées, l'inscription fluidique de l'histoire de la Terre. ${ }^{43}$

Also lückenlos fließende Einschreibung, Aufzeichnung der Erdgeschichte auf einem Lichtstrahl, der aus übereinandergelegten einzelnen Bildern besteht? Es sei nicht undenkbar, so Flammarion an anderer Stelle, dass der Bilderstrom auf einen dunklen Stern stoßen könnte, dessen lichtempfindliche Oberfläche aus Jod die Ausstrahlungen der fernen Erde wie in einer Photographen-Dunkelkammer zu fixieren vermöge. Wenn ein solcher astre obscur auch noch Zylinderform hätte und angemessen rotierte, dann hätte man eine unzerstörbare Bildersäule (colonne impérissable), „,sur laquelle se graveraient et s'enrouleraient d'eux-mêmes les grands événements de 1'histoire terrestre [... $]^{\text {“. }}{ }^{44}$ Für solch stellare Dunkelkammerphantasien haben nicht nur die Apparate-Technologien des 19. Jahrhunderts Pate gestanden, sondern auch altehrwürdige Bildrollenträger

41 Flammarion, Camille: Récits de l'infini. Lumen. Histoire d'une comete. Dans l'infini, Paris 1873, S. 143.

42 Eberty (wie Anm. 18), S. 21.

43 Flammarion (wie Anm. 41), S. 108.

44 Flammarion (wie Anm. 41), S. 137. 
vom Typus der Trajans-Säule in Rom oder der Pariser Vendôme-Säule (die 1871 gestürzt und dann wiedererrichtet worden ist). Auch dieser doppelte Bezug macht deutlich, wie Flammarions wiederholte Rede von Photographien zwischen optophysikalischen Bilderstrahlen und chemisch-stofflichen Reproduktionen oszillierte.

\section{Un microscope qui grossirait le temps}

Gingen Ausschmückungen wie die Idee einer belichtbaren stellaren Bildersäule, die Edisons Walzenphonographen von 1877 so schön antizipierte, eigene Wege, so waren andere Übernamen aus der Ebertyschen Vorlage um so deutlicher: So hat Flammarion das Mikroskop für die Zeit nicht nur wörtlich zitiert, sondern gleich auch noch eines der begleitenden Demonstrationsbeispiele vorausgeschickt. Eberty hatte, um die Vorteile der zeitmikroskopisch verlangsamten Bilderwahrnehmung auszumalen, das Phänomen des Blitzes in den Zeugenstand geholt.

Der Blitzstrahl z.B. erscheint uns als ein momentanes Leuchten, welches uns blendet, ohne eine Unterscheidung der Vorgänge, welche dieses Leuchten bewirken, zuzulassen. - Könnten wir aber dem Bilde eines solchen Blitzstrahles nur bis zur Sonne hinauf, durch acht Minuten folgen, so würden sich uns über die Natur dieses Phaenomens Geheimnisse erschließen, welche in ihrer Art gewiß nicht minder staunenswerth wären, als die lebendigen Welten, die das Mikroskop uns in Wassertropfen offenbart. ${ }^{45}$

Bei Flammarion lesen sich die entsprechenden (erheblich weiter ausgebreiteten, hier radikal gekürzten) Passagen so:

Si vous partiez de la Terre au moment où un éclair jaillit, et que vous voyagiez pendant une heure ou davantage avec la vitesse de la lumière, vous verriez l'éclair pendant aussi longtems que vous le regarderiez. [...] Or, si au lieu de voler juste avec la même vitesse que la lumière, vous aviez volé un peu moins vite, et que, par exemple, vous ayez employé un millième de secone de plus pour arriver au même point, au lieu de voir toujours le même moment de l'éclair, vous auriez vu successivement les divers moments qui constituent la dûrée totale de l'éclair, égale à un millième de seconde. Dans cette minute entière, vous auriez eu le temps de voir d'abord le commencement de l'éclair, d'en analyser le développement, les phases, et la suite jusqu'à la fin. Concevez alors quelles étranges découvertes on pourrait faire dans la nature intime de l'éclair grossi 60,000 fois dans l'ordre de là durée! Quelles batailles effrayantes vous auriez le temps d'apercevoir dans ses

45 Eberty, Felix: Die Gestirne II, London 1847, S. 3. 
flammes! Quel pandémonium! Quels sinistres d'atomes! Quel monde caché par sa fugacité aux yeux imparfaits des mortels! $[. . .]^{46}$

Auf derart eloquente Schilderung der minutiösesten Geheimnisse der Blitzerscheinung ließ Flammarion dann den Hinweis auf das Mikroskop für die Zeit folgen; oder, genauer gesagt: einen eigenen Verbesserungsvorschlag aus dem Munde von LUMEN, der um so deutlicher die begriffliche Anleihe dokumentierte:

Je vois dans votre pensée que vous comparez ce procédé à celui d'un microscope qui grossirait le temps. C'est exactement cela. Nous voyons ainsi le temps amplifié. Ce procédé ne peut pas recevoir rigoureusement la dénomination de microscope, mais plutôt celle de chronoscope, ou de chrono-télé-scope (voir le temps de loin). ${ }^{47}$

\section{Hermann von Helmholtz}

Chronoskop nannte man die im 19. Jahrhundert entwickelten Instrumente zur genauen Bestimmung des Eintritts von Erscheinungen oder zur Messung der Dauer von Vorgängen; im einfachsten Fall waren das StopUhren mit zusätzlichen, manuell schaltbaren Sekundenlaufwerken. Zur Aufzeichnung von Messdaten dienten unter anderem Zylinder-Chronographen, die in unserem Zusammenhange natürlich auch wieder an Flammarions photographische Sternbildsäule denken lassen. Während Flammarion also unter Apparateaspekten mit seinem chrono-télé-scope einerseits die alte Ressortverteilung der Leitinstrumente Mikroskop/Teleskop wieder anvisierte, war er wohl auch schon auf dem gedanklichen Wege zur Chronophotographie, die sein Landsmann Etienne Jules Maray als Begriff etabliert und als phototechnisches Verfahren perfektioniert hat.

In Deutschland hatte die Rede vom Mikroskop für die Zeit schon kurz nach dem Erscheinen der Eberty-Schriften weitere Kreise gezogen. Im Dezember 1850 hielt Hermann von Helmholtz in der physikalischökonomischen Gesellschaft von Königsberg einen Vortrag Ueber die Methoden, kleinste Zeittheile zu messen, und ihre Anwendung für physiologische Zwecke. ${ }^{48}$ Sein Interesse war auf die Messung ,schnell vorüber-

46 Flammarion (wie Anm. 41), S. 226-227.

47 Flammarion (wie Anm. 41), S. 228.

48 von Helmholtz, Hermann: „Ueber die Methoden, kleinste Zeittheile zu messen, und ihre Anwendung für physiologische Zwecke“, in: Königsberger Naturwissenschaftliche Unterhaltungen, 2. Bd., 2. Heft, Königsberg 
gehender Vorgänge des lebenden Körpers" - nämlich die Laufzeiten von Nervensignalen - gerichtet, die der menschlichen Wahrnehmung wegen ihrer Geschwindigkeit bislang entgangen waren. Helmholtz hatte die messtechnischen Fortschritte der vierziger Jahre sehr aufmerksam verfolgt und fasste sie in die Worte zusammen: „Sie sehen, dass die Mikroskopie der Zeit die des Raumes bei weitem überflügelt hat.“" ${ }^{49}$ Ist die Mikroskopie der Zeit also ein geflügeltes Wort gewesen, das um die Jahrhundertmitte in deutschen Wissenschaftlerkreisen zirkulierte und vom Berliner Astronomie-Liebhaber Eberty frühzeitig aufgeschnappt wurde? Oder wäre es tatsächlich denkbar, dass der Berliner Jurist, der über vielfältige gesellschaftliche Kontakte verfügte, das Mikroskop für die Zeit selbst in Umlauf gebracht hat? Angesichts der Originalität seiner Spekulationen kein völlig abwegiger Gedanke. - Jedenfalls ist vorläufig festzuhalten, dass sein Bonmot nicht nur in Frankreich auf fruchtbaren Boden gefallen ist, sondern auch im englischsprachigen Bereich weltweit Verbreitung gefunden hat.

\section{William von Voigts-Rhetz}

Noch einmal zurück zu Flammarions Récits de l'infini respective Lumen und der Frage, wie er vom Ebertyschen Text erfahren haben kann. In seinen Memoiren hat Flammarion 1911 behauptet, die Idee zu dieser Himmelsgeschichte sei ihm eines Abends 1865 ganz plötzlich beim Blick aus dem Fenster gekommen. ${ }^{50}$ Das mag durchaus so gewesen sein, aber es kann sich nur um ein Wiederauftauchen gehandelt haben. Bereits vor 1859 hatte W(illiam) von Voigts-Rhetz - Bruder des bekannten preußischen Generals und Militärchevalier an der Pariser Botschaft, der wie Flammarion in Spiritistenkreisen verkehrte - die anonyme englische Version der Eberty-Schrift in die Hände bekommen und begeistert eine Rückübersetzung ins Deutsche vorgenommen, die dann von Costenoble in Leipzig unter dem Titel Die Sterne und die Erde. Gedanken über Raum Zeit und Ewigkeit verlegt wurde. Aus dem Vorwort des Übersetzers wird deutlich, mit welchem Enthusiasmus der Text in Paris aufgenommen worden war: „Als der Zufall uns mit dem vorliegenden klei-

1851, S. 169-189, S.175 f. Ein spezieller Dank geht an Wolfgang Pircher (Wien) für die Überlassung seines Manuskripts zum Thema Gleichzeitigkeit.

49 Helmholtz (wie Anm. 48), S. 177.

50 Flammarion, Camille: Mémoires biographiques et philosophiques d'un astronome, Paris 1911, S. 331-334. 
nen Werke bekannt werden ließ,“ so Voigts-Rhetz, ,waren wir dergestalt von der Kühnheit und Originalität der darin enthaltenen Gedanken und Folgerungen überrascht, dass es uns ein dankbares Unternehmen schien, dasselbe auch dem mit Englischer Sprache und Literatur weniger vertrauten Theile des Deutschen Publicums zugänglich zu machen. Wie bedeutendes Aufsehen diese sich durchaus von den gewöhnlichen und ausgetretenen Bahnen des Gedankens entfernende, ihren eigenen und zugleich ganz eigenthümlichen Weg durch ein bis jetzt als mehr oder weniger unnahbar betrachtetes Gebiet verfolgende Schrift im Vaterlande des ungenannten Verfassers gemacht haben müsse, beweist hinlänglich die Zahl der in kurzer Zeit von derselben erschienenen Auflagen." Eine zusätzliche Fußnote präzisierte: „Die vorliegende Uebersetzung ist nach der sechsten in 11.000 Exemplaren erschienenen Auflage verfaßt worden."

Eine Teilauflage der Voigts-Rhetzschen Rückübersetzung erschien mit einem Hinweis auf den eigentlichen Autor Felix Eberty und einer Vorbemerkung des Verlegers Costenoble:

Der auffallende Zusatz zu dem Titel dieser kleinen Schrift erklärt sich dadurch, daß der Buchhändler Baillière in London gegen den erklärten Willen des Verfassers dessen Werkchen ins Englische hat übersetzen lassen, ohne weder auf dem Titel noch an irgend einer Stelle des Buches anzudeuten, daß er dem Publikum nicht ein englisches Originalwerk, sondern eine Übersetzung aus dem Deutschen vorlege. Dieses Verfahren des englischen Verlegers war die Veranlassung, daß weder der jetzige Verleger, noch der Uebersetzer von dem wahren Sachverhältniß Kenntnis erhielten, da das vor 13 Jahren erschienene deutsche Original ihrer Aufmerksamkeit entgangen war. Sie glaubten dem deutschen Publikum eine interessante Neuigkeit mitzutheilen, und erst nachdem diese Uebersetzung, die sich nunmehr als eine Rückübersetzung herausstellt, bereits gedruckt und versendet war, erlangten sie Kunde von dem Namen des Autors [...].

Es folgte eine salbungsvolle Adresse an den deutschen Genius Eberty, die Costenoble mit dem Wunsche verband, dass wenigstens nun, nach dem höchst merkwürdigen Schicksal der Schrift, ihr geistreicher deutscher Verfasser in seinem Vaterlande eine gleiche Anerkennung wie im Ausland finden möge. ${ }^{51}$ - Das Resultat war jedenfalls, dass auch die Rückübersetzungen bald vergriffen waren.

51 Auf den 20. August 1859 datierte Vorbemerkung des Verlegers Costenoble, die offenbar nachträglich einem Teil der Auflage zusammen mit einem revidierten Titelblatt beigefügt wurde. Eberty, Felix: Die Sterne und die Erde. Gedanken über Raum, Zeit und Ewigkeit, Leipzig 1860. 
Eberty gelang es schließlich 1874, eine eigene überarbeitete Neuauflage herauszubringen; und er hatte im Vorwort weitere juristische Bizarrerien zu berichten:

Der ursprüngliche Verleger war nach Südamerika ausgewandert, das Verlagsrecht durch Verpfändung und Verkauf aus Einer Hand in die andere gegangen, so daß der Verfasser erst nach Jahrelangen Bemühungen durch die Gefälligkeit des Leipziger Buchhändlers Herrn Gebhardt jetzt in den Stand gesetzt wird, diese neue Ausgabe ins Leben treten zu lassen.

Die nun vollnamentlich autorisierte Neuausgabe erlebte drei Auflagen (mit den textidentischen Nachdrucken von 1923 und 1925 also fünf), aber für eine unverzügliche weltweite Anerkennung war es zu spät. Flammarions Bücher hatten längst die Erträge der Ebertyschen Ideen auf französische Konten umgeleitet, und als Richard Proctor, renommierter Astronom und Fachbuchautor, 1879 aufgefordert wurde, eine revised edition der englischen Version zu verfassen, blieb es bei der Anonymität, denn Proctor kam nicht auf den Gedanken, nach einer möglichen namensgezeichneten Ausgabe auf dem Kontinent zu fahnden, obwohl er voll des höchsten Lobes war: „It is one of the most strikingly suggestive books, and small though it is, one of the most remarkable of the present century. " 52

\section{Richard Proctor}

Ob der 1888, vier Jahre nach Eberty, verstorbene Proctor jemals die Identität des von ihm bewunderten Autors erfahren und vielleicht Aufzeichnungen darüber oder sogar Korrespondenzen hinterlassen hat, müssen weitere Nachforschungen klären. Jedenfalls hat das Verlagshaus Bailliere, das auch noch die Neuausgabe von 1880 besorgte, Proctor über die Umstände der englischen Erstpublikation wohlweislich im Unklaren gelassen. Proctor seinerseits hielt es für das Beste, so wenig Änderungen wie möglich zu machen und nur aktualisierende Fußnoten hinzuzufügen. Er hatte guten Grund für diesen respektvollen Umgang mit dem für ihn immer noch namenlosen Text:

I remember well the interest with wich I read it, when my esteemed friend the late Mr. William Longman called my attention to the

52 Im Vorwort zu The Stars and the Earth, or Thoughts upon Space, Time, and Eternity. Revised and enlarged, with Notes by Richard A. Proctor, London 1880. 
work as one I ought to study before I comleted my treatise, Other Worlds than Ours'. It was the study of this charming little book which induced me to add to that work of mine the closing chapter on the ,Control and Supervision" of worlds, wherein I used (with due acknoledgement) some of the thoughts suggested by the anonymous writer of ,The Stars and the Earth '.

Proctors erstmals 1870 erschienenes, dann vielfach wiederaufgelegtes Buch - es wurde noch bis in die 1930er Jahre in überarbeiteten Fassungen nachgedruckt - brachte im besagten Schlusskapitel, nach voraufgeschicktem Verweis auf The Stars and the Earth, unter anderem folgende „beautiful and striking conception“ des von ihm referierten anonymen Autors:

Suppose that a being armed with such powers of vision as we have imagined should watch from the neighbourhood of our earth the progress of some interesting event. If he then began to travel from the earth at a rate equal to that at which light travels, he would see one phase of the event continually present before him, because he would always be where the light-message recording that event was actually travelling. By passing somewhat less swiftly away, he would see the event taking place with singular slowness; while by passing away more swiftly he would see the event occurring in inverted order. Suppose, for example, he were watching the battle of Waterloo, he could gaze on the fine picture presented by the Imperial Guard as they advanced upon the English army, for hours, years, nay, for centuries or cycles; or he might watch the whole progress of the charge occurring so slowly that years might elapse between each step of the advancing column, and the bullets which mowed down their ranks might either seem unmoving, or else appear to wend their way with scarcely perceptible motion through the air; or, finally, he might so wing his flight through space that the Guard would seem to retreat, their dead men coming to life as the bullets passed from their wounds, until at length the Old Guard would be seen as it was when it began its advance, in the assured hope of deciding Waterloo, as it had decided so many hard-fought battles for its Imperial Chief. ${ }^{54}$

Der anfangs beschriebene Zeitlupeneffekt entsprach in der Tat, wie wir schon wissen, dem Ebertyschen Text. Aber die Passage von der schließlich umgekehrt ablaufenden Waterloo-Schlacht konnte Proctor nicht aus The Stars and the Earth entnommen haben; sie ist dort ebenso wenig wie im deutschen Original zu finden. Hat er sie also hinzugefügt, um dem

53 Proctor (wie Anm. 52).

54 Proctor, Richard A.: Other Worlds than Ours: The Plurality of Worlds studied under the Light of recent scientific Researches, London 1870, S. 309-310. 
plot seines Gewährsmannes noch mehr blutrünstige Lebhaftigkeit zu verleihen? - Die Angelegenheit ist kurios wie viele Details der schon skizzierten Publikationsgeschichte der Eberty-Schrift. Man stößt nämlich in Flammarions 1872 erstmals gesammelt publizierten Récits de l'infini auf das exakte Gegenstück, und zwar im zweiten, Refluum Temporis überschriebenen Récit, das laut Fußnote 1867 geschrieben wurde; hier das Text-Pendant im Auszug:

C'était bien Waterloo, mais un Waterloo d'outretombe, car les combattants étaient des ressuscités. De plus, singulier mirage, c'est à reculons qu'ils marchaient les uns contre les autres. Une telle bataille était d'un effet magique, qui m'impressionnait d'autant plus fortement, que je devinais voir l'événement lui-même et que cet événement était étrangement transformé en son image symétrique. Remarque non moins singulière: Plus on se battait, et plus le nombre de combattants augmentait; à chaque trouée què le canon faisait dans les rangs serrés, un groupe de morts ressuscitait immédiatement pour boucher ces trouées. ${ }^{55}$

Hat Proctor also bereits die Zeitschriftenversionen der Flammarionschen Récits gekannt, und die darin enthaltene Waterloo-Episode dann versehentlich seiner älteren Quelle zugeschlagen? Oder hat Flammarion das 1870 erschienene Buch Proctors gekannt, als er seine Récits für die Buch-Edition von 1872 überarbeitete? - Die Angelegenheit muss einstweilen in der Schwebe bleiben; nur eines ist sicher: Beide Autoren haben den imaginären Zeitraffungen und -Dehnungen Ebertys eine signifikante Wendung hinzugefügt: die Zeitumkehr, den ,Rückfluss der Zeit', der sich im Gedankenexperiment einstellen musste, wenn hypothetische Beobachter mit Lichtgeschwindigkeit bewegte Bilderstapel zu überholen hatten.

Was Proctor - wie Eberty - von vornherein als immaterielle Erscheinungsweise der Bilder charakterisierte, hat Flammarion in seiner Waterloo-Vision zunächst als Erzählung von einer real ,verkehrten Welt` vorgeführt, um dann erst nachträglich die Auflösung des Rätsels à-laEberty anzubieten. Flammarion ist also seinen mutmaßlichen Vorbildern - z.B. den höchst pointenreichen, verkehrten Welten' die Gustav Theodor Fechner unter dem Pseudonym Dr. Mises 1824 und 1846 inklusive Grabausstiegen beschrieben hatte $\mathrm{e}^{56}$ - in der Handfestigkeit der Fiktion näher geblieben. Proctor dagegen hat wie Eberty die Ungreifbarkeit der

55 Flammarion (wie Anm. 41), S. 103.

56 Dr. Mises [Gustav Theodor Fechner]: „Verkehrte Welt“ [1824] und „Der Raum hat vier Dimensionen“" [1846], in: Kleine Schriften, Leipzig 1875. 
Bilder betont und sie damit im Grunde genommen nur noch der Überwachung zugänglich gemacht, aber der Kontrolle entzogen. Insofern benannte der letzte Kapiteltitel seines Buchs - Supervision and Control ein fundamentales theologisches Problem, das schon in Ebertys Entwurf aufgetaucht war: Gott konnte alles sehen; aber wie er durch Einblicke in bloße Bilder der Weltgeschichte letztere auf der Stelle hätte ,kontrollieren', das heißt, eingreifen sollen, blieb nach menschlichem Ermessen unerklärlich - und ist für weniger zuversichtliche Folgegenerationen zur Quelle zutiefst verstörender Tragik geworden.

\section{Carl du Prel}

Während sich im anglofranzösischen Sprachbereich eine besser bekannte Springflut von Zweitverwertungen und Paraphrasen des Flammarionschen Lumen-Stoffes anbahnte - die erste englische Übersetzung erschien bereits 1873 -, hat sich in Deutschland eine weniger spektakuläre Propagationslinie der Eberty-Schrift entwickelt, die gleichwohl für die Berliner Szene nach der Jahrhundertwende, auf die wir zum Schluss wieder zurückkommen, nicht unwichtig gewesen sein dürfte.

In der zweiten, vermehrten Auflage seines vielgelesenen Buchs Der Kampf ums Dasein am Himmel. Versuch einer Philosophie der Astronomie hat Carl du Prel 1876 - noch vor seiner ,Konversion“ zum Spiritismus - sehr direkt auf die Neuauflage der Eberty-Schrift von 1874 reagiert. In einer sechsseitigen Anmerkung sind die wesentlichen Aspekte referiert und kritisch gewürdigt: Ein Wesen, das im Weltenraum allgegenwärtig wäre, müsste in Bezug auf vergangene Dinge allwissend sein. Aber wenn auf diese Weise die Allwissenheit des Schöpfers gleichsam ihre naturwissenschaftliche Begründung erhalten solle, so wäre doch zu fragen, was das letztlich nütze, so du Prel: weil nämlich dabei das Wunder der Allgegenwart vorausgesetzt werden müsse. Ohnehin bleibe eine andere vorausgesetzte Eigenschaft des Allmächtigen, seine Kenntnis der Zukunft, gänzlich unerklärt. - Trotz derart prinzipieller Einwände war du Prel von Ebertys lichtbildwissenschaftlichen Ausführungen offenbar tief beeindruckt. Vor allem das Mikroskop für die Zeit war ihm eine prägnante Charakterisierung wert: Gleichwie für ein Auge, das sich mit entsprechender Geschwindigkeit einem Sterne nähere, die Begebenheiten auf demselben extrem zusammengedrängt würden, so könnten auch umgekehrt durch schnelle Entfernung die Ereignisse unendlich ausgedehnt werden. Es würden sich in Naturvorgängen Zwischenstadien entdecken lassen, die normalerweise unsichtbar blieben, und das könne zu neuen 
Erkenntnissen führen. Eine angenommene Allgegenwart würde nun wohl die gleichen Dienste leisten; ,aber die Erkenntnis selbst aller Zwischenstadien der Veränderungen bliebe doch fruchtlos ohne das Verständnis der zu Grunde liegenden Kräfte. ${ }^{\text {557 }}$ - Das entsprach exakt der rationalistischen Argumentationsweise des aufkommenden Monismus, dem du Prel mit seinem ,himmlischen“ Darwinismus zu kosmischer Geltung verhelfen wollte.

In der dritten, neuerlich überarbeiteten Auflage des Buchs, das 1882 nunmehr unter dem Titel Entwicklungsgeschichte des Weltalls. Entwurf einer Philosophie der Astronomie erschien, hat du Prel dem Mikroskop für die Zeit zusätzliche beispielhafte Leistungsmerkmale attestiert, die wiederum das notorische Problem der Motivwanderungen aufwerfen:

Einer Lokomotive könnte ein Schneckengang erteilt werden; durch genaue Anpassung der Bewegung [des Beobachters] könnte ein durch unsere Atmosphäre stürzender Meteorit gleichsam zum Stehen, ja, er könnte sogar zu einer rückwärtigen Bewegung gebracht werden. Ein [über die Möglichkeiten der Zeitmikroskopie verfügendes, kosmisches] Wesen könnte also die Zeit gleichsam rückwärts fließen lassen. ${ }^{58}$

Zitiert wurde Flammarion hier nicht, dafür aber kurz danach Karl Ernst von Baer mit seinen schon besprochenen Ausführungen über Naturanschauung.

Auch im bereits zwei Jahre zuvor publizierten Ergänzungsband über Die Planetenbewohner und die Nebularhypothese. Neue Studien zur Entwicklungsgeschichte des Weltalls hatte du Prel die exobiologische Seite der Baerschen Mutmaßungen sozusagen in Ebertyscher Perspektive weiter verfolgt:

Wesen, welche die Fähigkeit hätten, innerhalb einer Sekunde alle objektiven Veränderungen als subjektive Bewußtseinszustände zu empfinden, sodaß sie z.B. jede einzelne der Ätherschwingungen wahrnähmen, deren wir viele Millionen innerhalb einer Sekunde bedürfen, um eine Licht- und Farbenempfindung zu erhalten, würden gleichsam im Besitze eines Mikroskopes für die Zeit sein. [...] Wir können aber die Möglichkeit solcher Arten von Intelligenz noch aus anderen Voraussetzungen ableiten, die noch ganz im Gebiete des physikalisch Denkbaren liegen. [...] Nehmen wir nun an,

57 du Prel, Carl: Der Kampf um's Dasein am Himmel. Versuch einer Philosophie der Astronomie, Berlin 1876, S. 355.

58 du Prel, Carl: Entwicklungsgeschichte des Weltalls. Entwurf einer Philosophie der Astronomie, Leipzig, 1882, S. 376. 
es gebe Wesen, deren Organisation den Thätigkeitsweisen des Äthers entspräche, die z. B. ein der Lichtgeschwindigkeit analoges Bewegungsvermögen hätten, so könnten dieselben die eben erwähnte mikroskopische Auseinanderzerrung oder teleskopische Verdichtung zeitlich aufeinanderfolgender Veränderungen auch vermöge ihrer Bewegungskraft erzeugen. ${ }^{59}$

Das aber führte im Grunde zum Prinzip des Doppler-Effekts, ohne dass er direkt beim Namen genannt wurde:

Da nämlich Veränderungen der Dinge auf Bewegungen des Äthers beruhen, und es lediglich von der Anzahl seiner Schwingungen abhängt, auf welchen unserer Sinne sie wirken, d.h. ob sie als Töne, Wärme oder Licht wahrgenommen werden, so könnte ein auf einen Gegenstand hin oder von ihm abgewendetes Bewegungsvermögen auch dazu benützt werden, nach einander die verschiedenen Sinne von einem und demselben äußeren Gegenstande affiziren zu lassen; denn eine entsprechende Bewegungsgeschwindigkeit würde die Anzahl von Ätherschwingungen innerhalb einer Sekunde in der Annäherung vermehren, in der Entfernung vermindern. Ein solches Wesen könnte, wenn sein Gehör auf einen äußeren Vorgang mit einem tiefen Tone reagiren würde, diesen durch seine Bewegung in immer höhere Töne verwandeln, dann eine immer mehr gesteigerte Wärme-Empfindung erfahren, endlich den ursprünglichen Ton in eine Farbenempfindung umsetzen und das ganze Farbenspektrum durchlaufen lassen; oder es könnte auch durch die umgekehrte Reihenfolge der Empfindungen und, falls es noch andere Sinne für die uns nicht wahrnehmbaren Ätherschwingungen hätte, durch noch weitere Empfindungsmodalitäten bei identischer äußerer Ursache geleitet werden. ${ }^{60}$

Dieser Durchlauf der Sinne an einem einzigen vibrierenden Gegenstand war einem bekannten Gedankenexperiment Wilhelm Wundts nachgebildet, der einen schwingenden Stab sämtliche Frequenzen vom tiefsten akustischen Vibrieren bis über das sichtbare Leuchten hinaus hatte passieren lassen, um die Begrenztheit der einzelnen menschlichen Sinnesfenster aufzuzeigen. ${ }^{61}$

Du Prels Eingehen auf die Eberty-Schrift ist nicht nur wegen der darin dokumentierten exobiologischen Physiologisierung bemerkenswert, sondern auch, weil seine Schriften sich seinerzeit großer Verbreitung erfreuten, die heute erst in Umrissen wieder ermessbar wird. Eine weit ver-

59 du Prel, Carl: Die Planetenbewohner und die Nebularhypothese. Neue Studien zur Entwicklungsgeschichte des Weltalls, Leipzig 1880, S. 169-170.

$60 \mathrm{du}$ Prel (wie Anm. 59), S 170.

61 Wundt, Wilhelm: Vorlesungen über die Menschen- und Thierseele, 1. Bd, Leipzig 1863, S. 179. 
zweigte Schicht von Gebildeten, Künstlern und Literaten waren um die vorletzte Jahrhundertwende vom Gedankengut des monisitischen Spiritismus etc. beeinflusst, dessen profiliertester Vordenker Carl du Prel nach 1880 geworden ist. - Auch die Kenntnis der kleinen Eberty-Schrift, die du Prel zu den wichtigsten seiner Zeit zählte, ist sicher durch seine Publikationen befördert worden.

\section{Joseph Pohle}

Direkte Belege für solche Huckepack-Proliferation findet man auch bei den ausgemachten du Prel-Gegnern in kirchlichen Kreisen. So hat etwa Joseph Pohle (1852-1922), Theologieprofessor am Priesterseminar in Leeds, später Astronomie-Professor in Breslau, 1884/85 entschieden gegen du Prels ,darwinistische“ Astronomie Stellung bezogen, gleichwohl aber die Ebertyschen Bildgebungen übernommen und sogar noch weiter ausgeschmückt:

Setzen wir nun einmal den in sich nicht unmöglichen Fall, der Schöpfer hätte ein Auge geschaffen von so ungewöhnlicher Schärfe, so enormer Sehweite und so wunderbarer Empfindlichkeit, daß sich keine wie immer riesige Entfernung denken ließe, welche die Gesichtswahrnehmung dieses Wunderauges zu vereiteln imstande wäre. Es könnte mithin auch die Gegenstände und Ereignisse auf unserem Erdball aus beliebigen Entfernungen mit gleicher Schärfe beobachten und verfolgen wie wir. [...] Versetzen wir das Wunderauge ein Mal auf den äußersten Planeten Neptun [...] und lassen wir es von dort aus ein beliebiges geschichtliches Factum, z.B. die Schlacht von Waterloo in ihrem wechselreichen Verlauf, genau verfolgen. ${ }^{62}$

Die angegebene Quelle war in diesem Fall deutlich erkennbar Proctor, allerdings mit einigen Modifikationen, besonders kenntlich auch an der Verselbständigung des von Gott geschaffenen Wunderauges. ,Doch noch wunderbarere Scenen, als die vorgeführten, lassen sich bei einigen Abänderungen unserer optischen Fiction erzielen“, so Pohle weiter.

62 Pohle, Joseph: „Die Sternwelten und ihre Bewohner. Eine wissenschaftliche Studie über die Bewohnbarkeit und die Belebtheit der Himmelskörper nach dem neuesten Standpunkte der Wissenschaften. Erster Theil“, in: Jahresbericht der Görres-Gesellschaft für das Jahr 1884, Köln 1884; Joseph Pohle: „Die Sternwelten und ihre Bewohner. Eine wissenschaftliche Studie über die Bewohnbarkeit und die Belebtheit der Himmelskörper nach dem neuesten Standpunkte der Wissenschaften. Zweiter Theil“, in: Jahresbericht der Görres-Gesellschaft für das Jahr 1884, Köln 1885, Zitat S. 16. 
Denken wir uns das Riesenauge, statt in betrachtender Ruhe, in Bewegung, und setzen wir zunächst den Fall, dasselbe komme aus der unermeßlichen Ferne von mehreren tausend Lichtjahren in gerader Richtung und mit rasender Geschwindigkeit auf unsere Erde zu: so ist klar, daß die erste Scene, welche sich ihm am Beginn seiner Reise darböte, ein uraltes Ereigniß der Erdgeschichte, z.B. den Anfangs- und Urzustand des ersten Menschen, und die letzte Scene den gegenwärtigen Entwickelungspunkt der Menschen- und Erdgeschichte darstellen würde. [...] Dem Wunderauge würde während seiner Riesenreise in Wahrheit die ganze Erdund Weltgeschichte von ihren ersten Anfängen bis herab zur Gegenwart in rasch wechselnden Tableaux vivants aufgerollt, und zwar schneller oder langsamer je nach der Geschwindigkeit, mit welcher es sich durch den Raum bewegte. Würde die Reise in einem Augenblick erfolgen, so rasch wie ein Gedanke, so würde dieser eine bedeutende Augenblick, wie in einem magischen Bild, die lange Kette der bedeutendsten wie geringfügigsten Ereignisse aller Jahrhunderte blitzschnell vor dem supponirten Auge vorüberziehen $[\ldots]{ }^{63}$

Proctor folgend unterschied Pohle drei Fälle bei sehr schneller Bewegung des Wunderauges: sie könne der des Lichtes gleich sein, oder größer, oder kleiner. Im ersten Fall würde das Bild, mit dem das Wunderauge seine Reise anträte, nicht mehr von der Netzhaut weichen. Danach war du Prel mit der Schneckengang-Lokomotive und dem rückwärts stürzenden Meteoriten zitiert. Und dann folgte noch ein weiterer namentlich gekennzeichneter Quellenbezug:

Geradezu parodox aber würden die Wahrnehmungs-Verhältnisse ausfallen, wenn sich das Auge in einem schnelleren Tempo bewegte als das Licht: dann müßte nämlich der imaginäre Fall eintreten, daß die Ereignisse sich umkehrten. Die Erd- und Menschengeschichte würde auf den Kopf gestellt. Die Menschen würden zuerst auf dem Todesbette, dann auf dem Krankenlager, sodann im kräftigsten Mannes- und Jünglingsalter, und zuletzt als Säuglinge in der Wiege gesehen werden. Die Vergangenheit würde sukzessive, aber in umgekehrter Reihenfolge wieder zur Gegenwart. Denn das Auge würde ja infolge seiner schnelleren Bewegung alle diejenigen Lichtstrahlen der Reihe nach einholen, welche mit den älteren Nachrichten noch unterwegs wären. Wir hätten eine Verzerrung der Ereignisse, eine scheinbare Umkehrung der Zeit vor uns, in Wahrheit eine ,verkehrte Welt‘. ${ }^{64}$

Für diese Textpassage hat Pohle ausdrücklich per Fußnote Ebertys Schrift von 1874 angegeben. Doch der referierte Inhalt belegt: sie ist

63 Pohle (wie Anm. 62), S. 15-16.

64 Pohle (wie Anm. 62), S. 17. 
wohl nur aus der Erinnerung zitiert und mit Einsprengseln aus anderen Quellen durchsetzt; offenbar auch aus Flammarions Récits, ohne dass diese jedoch genannt sind.

Pohles Darlegungen sind auch deswegen so bemerkenswert, weil seine bescheidene wissenschaftliche Studie über die Bewohnbarkeit der Himmelskörper, die ursprünglich 1884/85 in den Jahresberichten der Görres-Gesellschaft erschienen war, dann als laufend aktualisierte Einführung in die moderne Astronomie beachtliche Auflagenzahlen erreichte. ${ }^{65}$ Noch $1922 \mathrm{kam}$ der siebte, vielfach erweiterte Neudruck des offenbar zugkräftigen Titels heraus. Aber die hier im Auszug zitierten Passagen, die nach wie vor unter der Kapitelüberschrift Die Gestirne und die Weltgeschichte, also unter dem Titel der Eberty-Schrift standen, waren nahezu unverändert geblieben.

Ebenso bemerkenswert wie die dauerhafte Verbreitung des Buchs war eine kleine, aber signifikante Paraphrasierung der ,zitierten ' Quellen: Bei Pohle war nicht mehr von Gott selbst oder anderen kosmischen Beobachtern die Rede, sondern von einem separaten Wunderauge, vor dem die Geschichte in Tableaux vivants, in magischen Bildern abgerollt wird. ${ }^{66}$ Die psychische Augenzeugenschaft hatte sich zu einer fast schon apparativen verwandelt. Man brauchte nur noch anstelle von Wunderauge vom Auge der Kamera zu sprechen, um den Schwenk zur phantasmagorisch-medialen Bühnenfiktion ins Blickfeld zu bringen.

\section{Berliner Urania}

Wie die Real-Phantasmagorie einer gerafften Urgeschichte in den achtziger-neunziger Jahren ablief, kann man sich noch zum Beispiel anhand der Libretti der Berliner Urania vor dem geistigen Auge auszumalen versuchen. So findet sich etwa in den Partituren der Bildervorträge, die M. Wilhelm Meyer seit 1888 im wissenschaftlichen Theater der Gesellschaft Urania hielt, der folgende aufschlussreiche Prolog zu einer Wanderung durch vorsintflutliche Landschaften.

(Regieanweisung: bei geschlossenem Vorhange): „Nichts ruht in der Welt, alles bewegt sich, alles entwickelt sich, alles strebt höheren, schöneren Zielen entgegen. [...] Ein Geist, der Millionen Jahre überblicken könnte so schnell, wie an uns eine Minute vorübergeht,

65 Pohle (wie Anm. 62). Die 3. Auflage erschien 1902, die 6. Auflage 1910; von der 2. Auflage an unter dem Titel: Die Sternenwelten und ihre Bewohner. Eine Einführung in die moderne Astronomie.

66 Pohle (wie Anm. 62), S. 16. 
würde die Sterne, diese gewaltigen Sonnen, um welche sich wie nachtschwärmende Falter die Planeten kreisend scharen, durch einander schwirren sehen, wie die Leuchtkäfer in einer lauen Juninacht. [...] Und so soll uns der Künstler, dessen Hand vom Geiste forschender Wissenschaft geleitet worden ist, zurückversetzen in die längst verschollenen Zeiten. [...] Wir wollen die große Geschichte der Erde durchwandern, die Zeit von Millionen Jahren mit Siebenmeilenstiefeln überfliegen in einer kurzen Stunde, die Sturm- und Drangzeit der irdischen Natur miterleben." (Regieanweisung: Der Vorhang öffnet sich) Erste Scene. Das Chaos. (Regieanweisung: Die Scene ist zunächst fast ganz finster. Die Nebel erglühen mehr und mehr und treiben lebhaft durcheinander. Dann sieht man die glühend flüssige Oberfläche der Erde, welche mehr und mehr erkaltet. Wasserdampf entwickelt sich und endlich sieht man die Erde vom Meere ganz überfluthet, dessen Grenzlinie sich am Horizonte aus den Nebeln scheidet. $)^{67}$

Die letzte der zwölf Szenen zeigte, nach diversen vorzeitlichen Landschaften, in einem ,Mittelmeergestade wie bei Sorent' mit antiken Ruinen die Ankunft in der kulturgeschichtlichen Gegenwart. Text und Bildregie waren sorgfältig verzahnt: Die Worte des Vortragenden überbrückten die Prospektwechsel bei geschlossenen Vorhängen, und die Szenenbilder wurden vermutlich mit dioramatischen Beleuchtungseffekten und/oder als dissolving views (mit ,Nebelbild'-Projektoren) verlebendigt. - Das Alles für die Einstundenvision der Weltgeschichte, die sich eventuell auch im bildhaften Ansatz von den astronomischen Phantasien eines Berliner Juristen herleiten lässt?

\section{Zwischenbilanz}

Was kann man demnach als Zwischenergebnis der hier skizzierten Odyssee des kleinen Eberty-Textes bis zu den Aufführungen des Berliner Urania-Wissenschaftstheaters festhalten? Vor allem Eines: Die kosmischen Perspektiven des Berliner Astronomie-Liebhabers waren nicht das sekundäre ,Abfallprodukt' einer Jahrmarktstechnologie, die lediglich an vulgären Bewegungsillusionen interessiert gewesen war. Im Gegenteil: Erst im Zusammentreffen mit einer instrumententechnischen Revolution - den ersten präzisen Fixstern-Parallaxenmessungen - zündete der Ebertysche Geistesblitz.

67 Meyer, Wilhelm M.: Die Geschichte der Urwelt. Eine Wanderung durch vorsintflutliche Landschaften, vorgetragen im wissenschaftlichen Theater der Gesellschaft Urania, Berlin 1890, S. 3-4. 
Denken wir uns, ,hatte Alexander von Humboldt 1845 im ersten Bande des Kosmos geschrieben,' als ein Traumbild der Phantasie, die Schärfe unserer Sinne übernatürlich bis zur äußersten Grenze des telescopischen Sehens erhöht, und zusammengedrängt, was durch große Zeitabschnitte getrennt ist, so verschwindet urplötzlich alle Ruhe des räumlichen Seins. Wir finden die zahllosen Fixsterne sich wimmelnd nach allen Richtungen gruppenweise bewegen; Nebelflecke wie kosmische Gewölke umherziehen, sich verdichten und lösen, die Milchstraße an einzelnen Punkten aufbrechen und ihren Schleier zerreißen; Bewegung eben so in jedem Punkte des Himmelsgewölbes walten, wie auf der Oberfläche der Erde in den keimenden, blättertreibenden, Blüthen entfaltenden Organismen der Pflanzendecke. ${ }^{68}$

Doch das war nur ein Vorspiel zu Humboldts schon zitierten Einsichten in die himmlische Vergangenheit gewesen.

Mit den ersten verlässlichen Ausmessungen von Sternabständen hatte sich nicht nur die schon lange vermutete zeitliche Tiefe des Weltenraums erhärtet, sondern auch der Blick zum Himmel als ein zwangsläufig in die Vergangenheit gerichteter erwiesen. Und dies musste ebenso für Beobachter auf anderen Sternen gelten. - Eberty hat als erster das ebenso plausible wie ideengeschichtlich folgenreiche Gedankenexperiment in Worte gefasst, einen fiktiven Augenzeugen, der letztlich niemand anderes als Gott sein konnte, die Vergangenheit der Erde von unterschiedlich weit entfernten Fixsternen zu entsprechend verschiedenen Zeiten beobachten zu lassen. Mehr noch: er ließ diesen allmächtigen Augenzeugen nicht nur in Ruhe zuschauen, sondern dachte ihn sich mit oder gegen den Strom der Lichtbilder bewegt, sodass nun auch Zeitraffungs- und Zeitdehnungseffekte zum Zuge kommen konnten. Ein Mikroskop für die Zeit nannte er das Prinzip der lichtbilderbegleitenden Zeugenschaft im Zeitlupen- oder Zeitraffertempo, dem sich die in den Weltenraum übertragene Babbagesche Idee von einem unzerstörbaren Kriminal-Archiv der Schallwellen hinzugesellte. - War damit, ein halbes Jahrhundert vor der technischen Realisierung, weitab von den üblichen Kategorienlehren der rein sukzessiv-zeitlichen oder koexistierendräumlichen Künste (Lessings zählebiges Erbteil), auch eine Art Denkmodell des ,kosmischen Kinos' ins Auge gefasst?

68 von Humboldt (wie Anm. 17), S. 155. 


\section{Himmelskino}

1988 erschien in der DDR ein Sonderheft der Beiträge zur Film- und Fernsehwissenschaft mit dem Schwerpunktthema Berlin - zwanziger Jahre, Zentrum filmtheoretischen Denkens. Unter den Aufsätzen: Die Utopie vom kosmischen Schauspiel und der Kinematograph des russischen Medienwissenschaftlers Michail B. Jampolski. ${ }^{69}$ Der Autor betonte in seinem Beitrag, dass der Stand des Filmbewusstseins auch gerade durchs Studium utopischer Filmtheorien geklärt werden könne. Damit brachte er den kosmischen Film, das so genannte Himmelskino zur Sprache, das besonders in den zwanziger Jahren viel Aufmerksamkeit erregt habe. Französische Filmtheoretiker wie André Imbert und Albert Bonneau hätten wie die deutschen Carl Hauptmann und Rudolf Harms damals davon gesprochen, dass der Film auf einer Bühne abrolle, die das Universum ist. Das sei keine exaltierte Metapher gewesen, sondern dahinter habe eine bedeutsame Tradition, ein bestimmter Stil des Denkens, eine besondere Filmutopie gestanden, die sich wohl bis zur deutschen Romantik, bis zum Siderismus Franz Xaver Baaders und Johann Wilhelm Ritters und zur Hieroastronomie Jean Pauls zurückverfolgen ließen. Dieser Ideenkomplex habe in Deutschland die spiritistische Paraastronomie Carl du Prels vorbereitet und sei in Camille Flammarions Lumen zur grandiosen Vorstellung eines kosmischen Kinematographen gesteigert worden.

Schon sechs Jahre zuvor, 1982, hatte Max Milner in seinem Buch La fantasmagorie die Tradition der Himmelslichtbilder ins Visier genommen. ${ }^{70}$ Für ihn war - nach Tiphaine de la Roche, der bereits 1760 eine reichlich hypothetische Fixiermethode für Lichtbilder beschrieben hatte - Eugène Mouton (1823-1902) mit seiner ,Phantasie“ Historioscope (1883) der prominenteste Vordenker gewesen; Flammarion folgte bei ihm noch zeitgleich als Ideenempfänger auf Platz zwei. Mittlerweile hat sich die Wertschätzung mit der genaueren Würdigung seiner Werke ganz zugunsten Flammarions gewendet: „Perhaps the single most innovative work in the entire literature of the scientific imagination", heißt es in ein-

69 Jampolski, Michail B.: „Die Utopie vom kosmischen Schauspiel und der Kinematograph", in: Beiträge zur Film- und Fernsehwissenschaft. Schriftenreihe der Hochschule für Film und Fernsehen der DDR ,Konrad Wolf“", 29, 34 (1988), S. 177-191. Ein spezieller Dank geht an Joachim Paech, der mich auf Jampolskis Text aufmerksam machte.

70 Milner, Max: La fantasmagorie. Essay sur l'optique fantastique, Paris 1982, S. $168 \mathrm{ff}$. 
schlägigen Übersichten. ${ }^{71}$ Und Entsprechendes scheint für die Literaturund Filmtheorie zu gelten: So findet man etwa in der Darstellung von Danielle Chaperon: Flammarion. Entre astronomie et littérature, Paris 1998, wesentliche Schlüssel- und Reizworte um den neuen Säulenheiligen der science fiction und Mediengeschichte versammelt: Nicht allein Le cinématographie céleste firmiert als Kapiteltitel, sondern auch $L a$ mémoire cosmique sowie La tristesse des astronomes und La mélancholie scientifique. $^{72}$

Die Traurigkeit der Astronomen und die wissenschaftliche Melancholie, die Danielle Chaperon so speziell Flammarion und seiner Zeit unterstellt hat, erweisen sich als Pendants einer bilderbezogenen Sehund Sehnsucht, die von Roland Barthes noch einmal auf eine poetisch knappe Formel gebracht worden ist:

Von einem realen Objekt, das einmal da war, sind Strahlen ausgegangen, die mich erreichen, der ich hier bin; die Dauer der Übertragung zählt wenig; die Photographie des verschwundenen Wesens berührt mich wie das Licht eines Sterns. Eine Art Nabelschnur verbindet den Körper des photographierten Gegenstandes mit meinem Blick: [...].

Und wenig später:

Die Photographie ruft nicht die Vergangenheit ins Gedächtnis zurück (nichts Proustisches ist in einem Photo). Die Wirkung, die sie auf mich ausübt, besteht nicht in der Wiederherstellung des (durch Zeit, durch Entfernung) Aufgehobenen, sondern in der Beglaubigung, daß das, was ich sehe, tatsächlich dagewesen ist. ${ }^{73}$

Das unumgänglich schmerzliche Distanzbewahren gegenüber der Vergangenheit war nun allerdings nicht gerade Flammarions Hauptanliegen in seinen Récits de l'infini gewesen. Seine LUMEN-Erzählungen hatten den Charakter von zwar spirituellen, aber durchaus noch leiblich motivierten Zeitreisen, die sogar intime Begegnungen mit geliebten Personen einbezogen. Daneben war der apparative Aspekt der lichtbilderfixierenden Sternsäule eher marginal (eine halbe Seite unter zweihundertsechsunddreißig in der Ausgabe von 1873). - Gleichwohl hat Jampolski betont, dass Flammarion speziell mit dieser kosmischen Aufzeichnungsap-

71 Magill, Frank N. (Hrsg.): Survey of Science Fiction Literature, Bd. 3, Englewood Cliffs, New Jersey 1979, S. 1294-1298.

72 Chaperon, Danielle: Camille Flammarion. Entre astronomie et littérature, Paris 1998.

73 Barthes, Roland: Die helle Kammer. Bemerkungen zur Photographie, Frankfurt a.M. 1985, S. 91 u. S. 92. 
paratur ein materielles Modell der ,Dauer' entworfen und so wesentliche philosophische Ideen Henry Bersons, nämlich das Kinematographenmodell des Bewusstseins, vorweggenommen habe. ${ }^{74}$ Flammarions QuÆRENS/LUMEN-Dialoge hätten die umfassendste und ausführlichste, sozusagen urtypische Utopie des kosmischen Films hervorgebracht, so Jampolski, der dann seinen Beitrag mit einer beeindruckenden Fülle von Nachfolgebeispielen: Scheerbart, El Lissitzky, Blaise Cendrars, Waleri Brjussow und anderen mehr, abgerundet hat.

Waren also Flammarions langatmige Récits und Ebertys Kurzgefasste Gedankenblitze tatsächlich im Wesentlichen konzeptionelle Vorformen der Kinematographentechnologie und damit Kronzeugen einer sich formierenden Kinomentalität vor dem Kino? Kann man damit einer Linie folgen, die Jampolski mit dem Hinweis auf Bergson angedeutet hat? - Statt sofort aufs spätere Kinematographenmodell aus Evolution créatrice von $1907 \mathrm{zu}$ setzen, sollte man sich besser zunächst an Bergsons Erstlingswerk Matiére et mémoire von 1896 halten, um den astronomischen Hintergründen seiner Weltsicht nachzuspüren.

\section{Henry Bergson}

In Matiére et mémoire hat Bergson sich als ,Bild unter Bildern“ beschrieben. Die anderen Bilder sah er unter der Einwirkung eines besonderen Bildes, nämlich des eigenen Leibes. Das hatte eine spezielle sinnesphysiologische Tradition: Für das Bewusstsein sei auch der Leib ein Teil der vorgestellten räumlichen Außenwelt, hatte Ewald Hering 1862 in seinen bahnbrechenden, gleichwohl heute kaum noch bekannten Beiträgen zur Physiologie geschrieben. ${ }^{75}$ Der Leib sei also selbst ein Vorstellungsbild, das laufend aus dem Gedächtnis vervollständigt und in den Sehraum mit hineingedacht werde, soweit nicht einzelne Teile wie Hand oder Fuß tatsächlich sichtbar seien. Und weiter: Inwiefern das Bild einer Raumwelt, welches wir bei offenen Augen träumen, bestimmt werde durch das jeweilige Netzhautbild, sei von der Physiologie rein empirisch festzustellen, so Hering. - In diesem Sinne auch Bergson: Als Realist gehe man von einer Gesamtheit, von einem Universum der Bilder aus, das kein Zentrum habe, sondern in dem sich alle Bilder ins Unendliche aneinanderreihten. Wenn alle diese Bilder dennoch auf ein bevorzugtes Bild [das des eigenen Leibes] bezogen würden, müssten sie sich mit jeder Körper-

74 Jampolski (wie Anm. 69), S. 179.

75 Hering, Ewald: Beiträge zur Physiologie. Von den identischen Netzhautstellen, Leipzig 1862, S. 166. 
bewegung umgruppieren. Sobald man jedoch ganz allgemein die Gegenwart an die Vergangenheit knüpfen und die Zukunft voraussehen wolle, müsse die egozentrische Anordnung wieder aufgegeben werden. Nur so sei die Wissenschaft vom Universum möglich. ${ }^{76}$ - Wie aber konnten menschliche Wahrnehmung und Gedächtnis in einer solchen Welt der Bilder funktionieren?

Die ganze Schwierigkeit des Problems, so Bergson, rühre daher, dass man sich die Wahrnehmung als eine Art photographischer Ansicht der Dinge vorstelle, welche von einem bestimmten Punkte mit einem besonderen Apparat - unserem Wahrnehmungsorgan - aufgenommen werde, um alsdann in der Gehirnsubstanz entwickelt zu werden. Nun liege aber, wenn man einen beliebigen Punkt im Weltall betrachte, die Sache so, dass die Wirkung der gesamten Materie ohne Widerstand und ohne Verlust hindurchgehe. Die mögliche Photographie des Ganzen bleibe [fließendes] Licht, denn es fehle die Platte zum Auffangen. Das menschliche Hirn müsse also mit seinen ,Zonen der Indeterminiertheit irgendwie die Rolle der Photoplatte übernehmen und auf seine Weise verwandeln. ${ }^{77}$ - Hat Bergson also in seinen einleitenden Überlegungen im Anfangskapitel von Matiére et mémoire bewegte Bilder gegen fixierte Photographien ausgespielt und damit zunächst das gerade Gegenteil vom Kinematographenmodell des Bewusstseins ins Auge gefasst? Bezeichnend scheint in unserem Zusammenhang die Durchdringung von Heringscher Optik und Flammarion-Ebertyschen Weltraumprospekten - bei spürbarer Reserve gegenüber rein mechanisch-apparativen (photographischen) Abbildungsverfahren. Das aber war im Ansatz eine eher wissenschaftsskeptisch antirationalistische Auffassung, wie sie der umstrittene Ausdruckstheoretiker Ludwig Klages dann so vehement in seinen Schriften verfolgt hat.

\section{Ludwig Klages}

In die Ferne gerückt könnten Gegenstände wenigstens zu Anschauungsbildern werden, schrieb Klages 1922 im Kosmogonischen Eros; wie auch umgekehrt gelte, dass aus der Unendlichkeit des schrankenlosen Weltraums Fernbilder näher und immer näher zu rücken vermöchten. Merkmal zur Unterscheidung der Nahbilder von entfernteren Gegenständen sei die Unantastbarkeit. Wenn es demgemäß zur Beschaffenheit der irdi-

76 Bergson, Henry: Materie und Gedächtnis. Eine Abhandlung über die Beziehung zwischen Körper und Geist, Jena 1919, S. 10-11.

77 Bergson (wie Anm. 76), S. 23-24. 
schen Körper schlechthin gehöre, der entfernten so gut wie der nahen, tastbar zu sein, so komme das Wesensmerkmal der Fernheit nur den Bildern des Himmels und erst vollends jedem zeitlich Vergangenen zu. Zeitliche Ferne erscheine in räumlicher Ferne; oder: das in der Ferne des Raumes Erscheinende sei Ferne der Zeit. Hier dürfe man ausnahmsweise, so Klages, „die Naturwissenschaft zu Hilfe rufen; pflegt uns doch die Astronomie zu belehren, schon vom Sirius, dem nächsten Fixstern, brauche das Licht, um bis zu uns zu gelangen, mehrere Jahre; daher wir denn, ihn erblickend, im Wahrnehmungsakt gegenwärtig hätten etwas um ebenso lange Zeit tatsächlich bereits Verflossenes! Indessen, auch wenn wir nicht gewiß zu sein glaubten, dass schon die Wissenschaft von morgen diese heutzutage volkstümliche Meinung als irrig verwerfen wird, so wäre gleichwohl alles verwirrt, vertauschten wir die ausmeßbare Entfernung des Siriuskörpers vom Erdplaneten mit dem Ferncharakter des Siriusbildes! Nicht von der Meilenlänge oder auch Lichtjahrlänge körpertrennender Abstände reden wir, sondern von jener Fernheit (gewissermaßen der Ferne an sich), die in der Schauung des Bildes unmittelbar (und somit vorbegrifflich) miterschaut wird.“78

Alles räumlich Ferne, so Klages, „kann in die Nähe rücken, bedingungslos ausgenommen allein die Gestirne! Mag unser Auge mittelst geschliffener Gläser noch um Lichtjahrtausende tiefer in die Schlünde des Raumes dringen und in der Dunkelkammer von Sternmyriaden das Abbild fangen an Himmelsstellen, wo sich vordem nur lichtlose Leere spannte, mögen wir [...] chemisch die Stoffe zerlegen der Sonnen und brennenden Dünste anderer Welten: wir haben nach wie vor gegenwärtig immer nur die Erscheinung der Sterne, niemals ihre (bloß erschlossene) Körperlichkeit! Darum denn: die Sterne die begehrt man nicht, und ihre Gegenwart, wenn uns ein Scherz erlaubt ist, um das über alles Vermuten Paradoxe daran zu beleuchten, glänzt durch Abwesenheit! - Könnte aber demgemäß kein überzeugenderes Sinnbild des Ehemals ersonnen werden als die Sternenpracht des nächtlichen Firmamentes, so begreifen wir nicht nur den Erhabenheitsschauer, der beim Anblick des funkelnden Gewölbes jeden noch welterschlossenen Betrachter durchhaucht, sondern wir verstehen auch, warum dem ursprünglichen Sinn die Sterne bald Seelen der Gewesenen, bald deren Aufenthaltsstätte waren, immer aber auf Erden vergangene im Unvergänglichen leuchtende Chöre der Vorwelt, an die aller irdische Wandel gebunden blieb. “79

78 Klages, Ludwig: Vom Kosmogonischen Eros, München 1922, S. 102.

79 Klages (wie Anm. 78), S. 111-112. 
Gemessen an der Aufbruchsstimmung und buchstäblichen Zuversicht, die sich in den Texten des 19. Jahrhunderts zeigten, klingen Klages' Berufungen auf die Astronomie merkwürdig resigniert und exaltiert zugleich. Tatsächlich projizierte er einen modernen Kenntnisstand, das Wissen um die in Lichtjahren zu messenden Fixsternabstände, zurück auf die angeblichen Erhabenheitsschauer vorgeschichtlicher Kulturen. Hatten die nicht jederzeit mit der Gegenwart ihrer Götter und Ahnen gerechnet?

\section{Eros der Ferne}

Einen irrationalen Schluss- und Fluchtpunkt solcher Himmelsperspektiven hatte Klages schon 1921 in seiner Schrift Vom Wesen des Bewußtseins gesetzt: „Nicht Dinge, sondern Bilder sind beseelt: das ist der Schlüssel zur ganzen Lebenslehre.“ Diesen Schlüssel aber könnten Naturwissenschaften nicht besitzen, weil sie anstelle der ursprünglichen Wirklichkeit der Bilder, so Klages, die abgeleitete Wirklichkeit bloß unterstellter Dinge setzten. ${ }^{80}$ Was also hatten die Bilder zu bieten? - Ihre Ursprünglichkeit erschien in Klages' psychologisch-philosophischen Frühschriften noch zusätzlich aufgeladen mit Sprachfiguren mehrsinnigen Empfindens:

Eine Hingegebenheit, die jeden Widerstand der Selbstbehauptung schmilzt, Fernblau über allen Gegenständen und ihr Eingebettetsein in einen Strom des Vergehens, in den wir mitversinken: aus diesen drei Fäden ist der Schleier gewoben, durch den der tagwache Geist die Welt ,wie im Traum‘ erblickt. ${ }^{81}$

So Klages über das Traumbewusstsein. In einer anderen Formulierung war mit dem Einschmelzen in die Welt der Bilder auch das Fernweh des kosmogonischen Eros schon angekündigt:

Aus immernaher Körperlichkeit vor eine ziehende Ferne gerückt verlöre das Ich seinen Halt auch ohne die Selbsthingebung, die es von innen wirkend nun vollends unter die Bilder stellt, dermaßen, daß es sich selbst mit ihnen verwechselt. ${ }^{82}$

Direkt synästhetische Qualitäten waren angesprochen, wenn er etwa behauptete, ,dass aber die Wahrnehmung des räumlich Fernen eine eigen-

80 Klages, Ludwig: Vom Wesen des Bewußtseins, Leipzig 1921, S. 28.

81 Klages, Ludwig: „Vom Traumbewusstsein“ [1913/1919], in: Mensch und Erde. Gesammelte Abhandlungen, Stuttgart 1956, S. 147-195, spez. S. 159.

82 Klages (wie Anm. 81), S. 159. 
tümliche ,Klangfarbe“ habe.“ ${ }^{83}$ Das multimodale Erleben komprimierte Klages schließlich im Eros der Ferne zum Gesamtkomplex einer unstillbaren Sehnsucht gegenüber den aus der Vergangenheit herüberleuchtenden Bildern. - Isis-Schleier und Nimbus waren die beiden aus derartiger Melancholie geschöpften Bezeichnungen, die Benjamins Aura-Begriff am nächsten kamen.

Über das Verhältnis der so gegensätzlichen Charaktere Benjamins und Klages' gibt es wenige, aber dafür aufschlussreiche Untersuchungen, die hier nicht eigens referiert werden müssen. ${ }^{84}$ Klar ist jedenfalls, dass Benjamin zentrale Begrifflichkeiten seiner Kunstwerkbetrachtung von Klages übernommen hat. In unserem Zusammenhang fällt auf, dass Benjamins berühmte Definition der Aura als ,einmalige Erscheinung einer Ferne, so nahe sie sein mag “, keine direkten Spuren ihrer Herkunft aus astronomischen Kontexten mehr verrät; im Gegenteil. Die multimodal-synästhetische Qualität der Unantastbarkeit, die Klages noch so ausdrücklich mit den in Abwesenheit glänzenden Sternen hinterlegte, hat sich bei Benjamin gleichsam vom Firmament abgenabelt und an den Kultobjekten der Menschheitsgeschichte niedergeschlagen. Und auch die anderen Wahrnehmungsbesonderheiten, die sich ursprünglich als imaginative Auswüchse der Astronomie gebildet hatten, ließ Benjamin ganz als irdische Errungenschaften der neuen technischen Medien zu Buche schlagen.

Was Eberty sich als mögliche Betrachtung davoneilender Lichtbilder zwischen den Sternen vorstellte, hat Benjamin - laut Anmerkung Arnheims prosaischen Filmemacherrezepten folgend - als bodenständig erzeugte Apparate-Illusionen genommen und ihnen eine eigene innere Hintergründigkeit nachgesagt:

Unter der Großaufnahme dehnt sich der Raum, unter der Zeitlupe die Bewegung. Und so wenig es bei der Vergrößerung sich um eine bloße Verdeutlichung dessen handelt, was man »ohnehin« undeutlich sieht, sondern vielmehr völlig neue Strukturbildungen der Materie zum Vorschein kommen, so wenig bringt die Zeitlupe nur bekannte Bewegungsmotive zum Vorschein, sondern sie entdeckt in diesen bekannten ganz unbekannte, , die gar nicht als Verlangsamungen schneller Bewegungen sondern als eigentümlich gleitende, schwebende, überirdische wirken.' So wird handgreiflich, daß es eine andere Natur ist, die zu der Kamera als die zum Auge spricht. Anders vor allem dadurch, daß an die Stelle eines vom

83 Klages (wie Anm. 78), S. 96.

84 Pauen, Michael: „Eros der Ferne. Walter Benjamin und Ludwig Klages“, in: Global Benjamin. Internationaler Walter-Benjamin-Kongress 1992, Bd. 2, S. 693-716. 
Menschen mit Bewußtsein durchwirkten Raums ein unbewußt durchwirkter tritt. [...] Hier greift die Kamera mit ihren Hilfsmitteln, ihrem Stürzen und Steigen, ihrem Unterbrechen und Isolieren, ihrem Dehnen und Raffen des Ablaufs, ihrem Vergrößern und ihrem Verkleinern ein. Vom Optisch-Unbewußten erfahren wir erst durch sie, wie von dem Triebhaft-Unbewußten durch die Psychoanalyse. $^{85}$

Könnte man - mit den hier gesammelten Indizien im Sinn - nicht auf den Gedanken kommen, dass Benjamins Rede vom Optisch-Unbewußten auch auf einen ihm entfallenen größeren Zusammenhang der ideellen Kamera-Vorgeschichte verweist, in dem Astronomie und Blickverhalten noch direkt verbunden waren und der mit dem Mikroskop für die Zeit einen griffigen Namen erhalten hatte?

\section{Engel der Geschichte}

Es gibt ein Bild von Klee, das Angelus Novus heißt. Ein Engel ist darauf dargestellt, der aussieht, als wäre er im Begriff, sich von etwas zu entfernen, worauf er starrt. Seine Augen sind aufgerissen, sein Mund steht offen und seine Flügel sind ausgespannt. Der Engel der Geschichte muß so aussehen. Er hat das Antlitz der Vergangenheit zugewendet. Wo eine Kette von Begebenheiten vor uns erscheint, da sieht er eine einzige Katastrophe, die unablässig Trümmer auf Trümmer häuft und sie ihm vor die Füße schleudert. Er möchte wohl verweilen, die Toten wecken und das Zerschlagene zusammenfügen. Aber ein Sturm weht vom Paradiese her, der sich in seinen Flügeln verfangen hat und so stark ist, daß der Engel sie nicht mehr schließen kann. Dieser Sturm treibt ihn unaufhaltsam in die Zukunft, der er den Rücken kehrt, während der Trümmerhaufen vor ihm zum Himmel wächst. Das, was wir den Fortschritt nennen, ist dieser Sturm. ${ }^{86}$

Der Rücklauf der Geschichte, den Benjamins Engel vergeblich anstrebe, schrieb Otto Karl Werckmeister 1997 über diese Linke Ikone ${ }^{87}$, erinnere an einen Text von Blaise Cendrars aus dem Jahre 1919: Das Ende der Welt, gefilmt vom Engel Notre-Dame. Das fiktive Drehbuch erreicht sei-

85 Benjamin, Walter: „Das Kunstwerk im Zeitalter seiner technischen Reproduzierbarkeit" (Dritte Fassung), in: Gesammelte Schriften, hrsg. v. Rolf Tiedemann/Hermann Schweppenhäuser, Bd. I.2, Frankfurt a.M. 1991, S. 500.

86 Benjamin, Walter: „Über den Begriff der Geschichte“, in: Gesammelte Schriften, hrsg. v. Rolf Tiedemann/Hermann Schweppenhäuser, Frankfurt a.M. 1991, Band I.2, S. 697-698.

87 Werckmeister, Otto Karl: Linke Ikonen. Benjamin, Eisenstein, Picasso nach dem Fall des Kommunismus, München/Wien 1997, S. 45. 
nen Höhepunkt, als besagter Engel vom Turm der Pariser Kathedrale die Posaune zum Weltende bläst, sodass Paris und alle anderen Städte der Erde zusammenstürzen und die Menschen unter Trümmern begraben. Doch dann läuft der Film rückwärts, die Toten erstehen auf, die Städte errichten sich wieder. Im Zeitraffer geht es zurück bis zum Weltanfang. Im letzten Abschnitt des Drehbuchs, überschrieben ,Gegen den Strich“ ( $A$ rebours), wird der Film der Weltgeschichte im Zeitraffer wieder vorgespult; bis zu der Szene, mit der er begann: Gottvater sitzt als hektisch zigarrerauchender Großunternehmer wieder in seinem Büro. „ECT“ heißt es zum Schluss, und: „C'est la banqueroute.“

Wie Cendrars zwischen dem Engel mit der Kamera und dem Menschen am Projektor, zwischen Drehen und Sehen des Films einen Unterschied machte, so Werckmeister, so habe Benjamin in seiner These zwischen dem Engel der Geschichte und uns unterschieden. Doch Cendrars' spielerisch-anarchistisches Hin und Her zwischen den Weltkatastrophen vor oder nach der Gegenwart habe Benjamin nicht nachvollzogen. - Und auch die höchst auffällige Körperwendung gegen die Bewegungsrichtung, so können wir hinzufügen, hätte Benjamin nicht von Cendrars übernehmen können. Der hatte nur das mechanische Umschalten der Filmlaufrichtung in einer Zwischenszene aus dem ,Projektorraum' kurz beschrieben, aber nirgends von einer bestimmten Haltung des Engels von Notre-Dame gesprochen. - Was also hat Benjamin zu seiner eigentümlichen Ausdeutung des Kleeschen Bildes bewogen?

In Flammarions QuÆRENS/LUMEN-Dialogen war das Problem, welche Körperhaltung ein mit den Lichtbildern bewegter Weltraumbeobachter einzunehmen hätte, direkt angesprochen worden:

Pour remonter ainsi les événements en vous éloignant dans l'espace, est-ce que vous voliez en reculant, ou plutôt les esprits sont ils doués dé la faculté dé voir derrière eux?

Antwort:

Quelle question! Si j'entreprenais de vous exposer par quel sens intime les esprits voient, je vous plongerais dans la discussion $\mathrm{d}$ un problème insoluble pour vous. Pour votre satisfaction personnelle, pensez que je me retournais de temps en temps pour examiner la Terre; cette idée sera plus facile à accepter. ${ }^{88}$

Hat Benjamin also irgendwann Flammarions Récits gelesen und daraus nicht nur die szenenprägende Körperorientierung des Engels, sondern

88 Flammarion (wie Anm. 41), S. 110. 
womöglich sogar das ganze Konzept der Geschichtsbetrachtung entlehnt? - Er hätte eine entsprechende, aber klarer formulierte Antwort auch in einer der vielen Auflagen von Pohles Sternenwelten, und diesmal mit ausdrücklichem Fußnotenhinweis auf Eberty, wie wir gesehen haben, finden können:

Am merkwürdigsten würde sich aber das Panorama für das fingierte [Wunder-]Auge gestalten, wenn wir dasselbe sich mit unermesslicher Geschwindigkeit von unserer Erde weg, obwohl stets behufs Aufnahme der Lichteindrücke zu ihr hingekehrt, in den Himmelsraum hinaus bewegen lassen. ${ }^{89}$

\section{Textevidenz}

Beim schrittweisen Durchgehen der Benjaminschen Bildbeschreibung seines ,Engels der Geschichte“ muss es wie Schuppen von den Augen fallen, wie klar durch die zeitgeschichtlich bedingten Verfärbungen und Verzerrungen hindurch die Bezüge zu den Textvorlagen des 19. Jahrhunderts sich abzeichnen: Der Engel starrt auf Etwas, von dem er sich rückwärts entfernt; sein Antlitz ist der Vergangenheit zugewendet. Das ist die bewegliche Position des lichtbilderbegleitenden Weltraumzeugen, wie sie zuerst von Eberty beschrieben wurde. Der Bezug zum Eberty-Text wird noch eindeutiger, wenn man die eigens von ihm hervorgehobene Wahrnehmungsform des Nebeneinander noch einmal vergleicht:

Hier haben wir also die Ausdehnung der Zeit mit der des Raumes zusammenfallend, der sinnlichen Anschauung so nahe gebracht, daß Zeit und Raum als gar nicht von einander geschieden begriffen werden können. - Denn: das in der Zeit nacheinander Folgende liegt hier räumlich gleichzeitig neben einander.“90

Das Gemälde, von dem Eberty sprach, hat sich für Benjamins Engel in die Simultanansicht einer einzigen Katastrophe, zum Trümmerhaufen, der zum Himmel wächst, verwandelt. Die freiwillige Beobachterbewegung, die bei Eberty zur, Gemäldeauffassung' der Zeit führte, wurde bei Benjamin zum ,Sturmwind des Fortschritts', der sich verselbständigt hat und nun den machtlosen Beobachter mit sich reißt. Der vergebliche Wunsch des Engels, zu verweilen, ist nicht anderes als das ins Gegenteil gekehrte Vermögen des Ebertyschen Augenzeugen, ,auf seiner Bahn beliebig anzuhalten“, um Momente der Geschichte in Muße zu studieren. ${ }^{91}$

89 Pohle (wie Anm. 62), S. 16. m. H.

90 Eberty (wie Anm. 18), S. 15-16.

91 Eberty (wie Anm. 18), S. 24-25. 
Der Engel möchte wohl verweilen, die Toten wecken und das Zerschlagene zusammenfügen, heißt es in Benjamins Beschreibung. Hier könnte man in der Tat zunächst an Cendrars rückwärts laufenden Film oder an Flammarions Waterloo d'outretombe denken. Aber eine solche emotionalisierte Anleihe war meiner Ansicht nach weniger grundlegend als die formale Übernahme der Weltraumzeugenschaft, die aus der Kette von Ereignissen ein Schreckensgemälde machte. Mit der Ohnmacht des Engels, die Geschichte zum Stehen zu bringen und einzugreifen, ist ein weiterer Aspekt, der auch schon im Ebertyschen Beobachterstatus gegeben war, bis zur äußersten Konsequenz getrieben: Der imaginäre Augenzeuge - und sei es sogar der Allmächtige selbst - kann nur noch zuschauen, Bilder inspizieren, aber nicht mehr nachträglich verändern. Mit dieser restriktiven Einsicht sind wir sozusagen beim visuellen Grundton der Geschichtsthesen Benjamins, der die hier verfolgte Tiefenperspektive der Motivwanderungen weiter unterstreicht.

Nimmt man sich den Text der übrigen Geschichtsthesen Benjamins der Reihe nach vor, so lässt sich eine Epitomensammlung zum hier diskutierten Motivkomplex fast als Fließtext zusammenfügen: Nichts, was sich jemals ereignet habe, sei für die Geschichte verloren zu geben. Aber erst der erlösten Menschheit sei ihre Vergangenheit in jedem ihrer Momente zitierbar geworden. (III) - Im Klassenkampf entfachte Eigenschaften wie Mut, Humor, List, Unentwegtheit wirkten in die Ferne der Zeit zurück. Das Gewesene seinerseits wende sich kraft eines Heliotropismus geheimer Art der Sonne zu, die am Himmel der Geschichte aufgehe. (IV) - Das wahre Bild der Geschichte husche vorbei. Nur als Bild, das auf Nimmerwiedersehen im Augenblick seiner Erkennbarkeit aufblitze, sei die Vergangenheit festzuhalten. (V) - Vergangenes historisch artikulieren heiße, sich einer Erinnerung zu bemächtigen, wie sie im $\mathrm{Au}-$ genblick von Gefahr aufblitze. (VI) - Es gelte, sich des echten historischen Bildes zu bemächtigen, das flüchtig aufblitze. Geschichte sei Gegenstand von Konstruktionen, deren Ort nicht die homogene und leere Zeit sei, sondern die von Jetztzeit erfüllte. So sei für Robespierre das antike Rom eine mit Jetztzeit geladene Vergangenheit gewesen, die er aus dem Kontinuum der Geschichte heraussprengte. Das sei der Tigersprung ins Vergangene. Denselben Sprung unter dem freien Himmel der Geschichte habe Marx als den dialektischen der Revolution begriffen. (XIV) - Das Bewusstsein, das Kontinuum der Geschichte aufzusprengen, sei den revolutionären Klassen im Augenblick ihrer Aktion eigentümlich. Revolutionäre Kalenderumstellungen fungierten dabei als historische Zeitraffer. (XV) - Auf den Begriff einer Gegenwart, die nicht Übergang 
sei, sondern in der Zeit einstehe und zum Stillstand gekommen sei, könne der historische Materialist nicht verzichten. (XVI) - Zum Denken gehöre nicht nur die Bewegung der Gedanken, sondern ebenso ihre Stillstellung. Es gelte die Zeichen einer messianischen Stillstellung des Geschehens zu erkennen. (XVII) - Die Jetztzeit, die als Modell der messianischen in einer ungeheuren Abbreviatur die Geschichte der ganzen Menschheit zusammenfasse, falle haarscharf mit der Figur zusammen, die die Geschichte der Menschheit im Universum mache. (XVIII)

Vorbeihuschen und Aufblitzen einerseits, Stillstellen und Aufsprengen andererseits; - das sind wörtliche Gegensatzpaare, die Benjamin als menschliche Erfahrungs- und Behandlungsweisen von Geschichte bzw. Geschichtsbildern angeboten hat; sie suggerieren gleichermaßen eine besondere Positionierung des wahrnehmenden Zeitzeugen, und sie lassen unmittelbar an Ebertys und Flammarions ausgedehnte Blitz-Betrachtungen denken. Den Wortfiguren nach befindet sich der Zeuge neben oder gegenüber dem Bilderstrom der Geschichte, der blitzartig an ihm vorübergleitet und den er stillstellen und aufsprengen muss, um zu Einsichten zu gelangen. Was der Engel der Geschichte als Ganzes überblickt, aber nicht mehr ändern kann, lässt sich von Menschen in revolutionären Augenblicken der ,messianischen Stillstellung ' als Erkenntnis verbuchen. - Diese erhellende Einsichtsfähigkeit, in der Benjamin persönliche Tragik und Trost zugleich formulierte, führt uns abschließend noch einmal zu der Frage, wie sich das Verhältnis von Augenzeugen und Zeitstrom im Längsschnitt und in den Verzweigungen der imaginären Weltraumperspektiven entfaltet hat.

\section{Augenzeugen}

1949 hat Albert Einstein in seinen Autobiographischen Notizen von einem Paradoxon berichtet, auf das er schon mit sechzehn Jahren, also um 1894, gestoßen sei:

Wenn ich einem Lichtstrahl nacheile mit der Geschwindigkeit c (Lichtgeschwindigkeit im Vacuum), so sollte ich einen solchen Lichtstrahl als ruhendes, räumlich oszillatorisches elektromagnetisches Feld wahrnehmen. So etwas scheint es aber nicht zu geben, weder auf Grund der Erfahrung noch gemäß den Maxwell'schen Gleichungen. Intuitiv klar schien es mir von vornherein, daß von einem solchen Beobachter aus beurteilt alles sich nach denselben Gesetzen abspielen müsse wie für einen relativ zur Erde ruhenden Beobachter. Denn wie sollte der erste Beobachter wissen bzw. konstatieren können, daß er sich im Zustand rascher gleichförmiger 
Bewegung befindet? Man sieht, daß in diesem Paradoxon der Keim zur speziellen Relativitätstheorie schon enthalten ist. ${ }^{92}$

Einsteins Jugenderinnerung besticht nicht nur durch das lebhaft leibhaftige Wortbild, dass man persönlich einem Lichtstrahl nacheilt, um ihn ,in Ruhe' zu betrachten; dieser Gedankenausflug erinnert mit der fingierten Parallelbewegung des Augenzeugen zum Lichtstrom auch direkt an die hier durchgegangenen Vorläufertexte. Fazit: Einstein hat nicht nur nachweislich aus Bernsteins Volksbüchern die Ebertysche Idee einer lichtbilderbegleitenden Zeugenschaft sich aneignen können; er hat sie auch direkt als Denkfigur übernommen und zum Sprungbrett seiner Physik-revolutionierenden Überlegungen gemacht. ${ }^{93}$

An dieser Stelle mag ein weiteres Gedankenexperiment Einsteins die Ausgangslage seiner (speziellen) Relativitätstheorie von 1905 - und die anschauliche Nähe zu den früheren fiktiven Weltraumperspektiven ansatzweise verdeutlichen: Angenommen ein Zug führe mit konstanter Geschwindigkeit an einem neben den Schienen stehenden Beobachter vorbei. Ein zweiter Beobachter befinde sich genau in der Mitte des vorbeifahrenden Zuges. Gerade zu dem Zeitpunkt, zu dem der im Zug sitzende Beobachter an dem draußen stehenden vorbeifährt, beobachten beide das gleichzeitige Eintreffen zweier Lichtsignale, die vom Anfang und vom Ende des Zuges kommen. Welche Schlussfolgerungen ziehen nun beide Beobachter hinsichtlich der Zeitpunkte, zu denen die Lichtsignale am Anfang und Ende des Zuges ausgesandt worden sind? - Der fahrende Beobachter berücksichtigt, dass er in der Mitte des Zuges steht und dass die Lichtgeschwindigkeit unabhängig vom Bewegungszustand ist. Daraus schlussfolgert er, dass beide gleichzeitig bei ihm eintreffenden Lichtsignale auch gleichzeitig von beiden Endpunkten des Zuges ausgegangen sind. - Auch der neben den Geleisen stehende Beobachter weiß, dass die Lichtgeschwindigkeit konstant ist und dass ein Lichtsignal zum Zurücklegen eines endlichen Weges endliche Zeit benötigt. Aus der Gleichzeitigkeit des Eintreffens beider Lichtsignale schlussfolgert er jedoch, dass das Signal am Zugende eher gegeben wurde, da das Zugende zum Zeitpunkt des Aufleuchtens weiter von ihm entfernt gewesen sein muss als der Zuganfang. Der vom Zuganfang ausgehende und sich mit

92 Einstein (wie Anm. 6), S. 48-50.

93 Derzeit neuester Forschungsstand mit Schwergewicht auf kulturtechnischen Aspekten bei Galison, Peter: Einstein's Clocks, Poincaré's Maps. Empires of Time, NewYork 2003. Sehr informativ in der Problemtiefe: Pais, Abraham: „Subtle is the Lord...". The Science and the Life of Albert Einstein, Oxford 1982. 
konstanter Geschwindigkeit ausbreitende Lichtimpuls kann nur dann zugleich mit dem vom Zugende ausgehenden ankommen, wenn er später erzeugt worden ist. Der ruhende Beobachter folgert somit, dass beide Ereignisse zu unterschiedlichen Zeiten stattgefunden haben müssen. ${ }^{94}$ - So weit die Schilderung der Augenzeugenurteile, denen dann eine rigorosere mathematische Fassung und Fortsetzung folgte.

In seiner die Relativitätstheorie begründenden Abhandlung war zunächst ,nur' von bewegten Stäben, gleichwohl aber von Lichtlaufzeiten die Rede. ${ }^{95}$ Im Prinzip entsprachen Einsteins Minimalmodelle durchaus den großen interstellaren Imaginationsprospekten, wie sie von Eberty aus der Taufe gehoben und von Flammarion und Anderen ausgewalzt worden sind. Man hat also nur an die Stelle des Eisenbahnzuges die fiktiven Lichtbilderströme (sozusagen die erleuchteten Fenster der Waggons, die sich dann auf zwei Lichtsignale reduzierten) zu setzen, um ein anschauliches Leitbild der von Einstein entwickelten Theorie vor Augen zu haben.

Nicht nur relativistische Probleme wurden von der fingierten Beobachterpositionierung aus angegangen; es gab auch anthropologische: Ruhe und Bewegung, voraus- bzw. zurück- oder aber seitwärts gerichteter Blick; das sind offensichtlich die grundsätzlich möglichen Posen menschlicher Betrachterorientierung. Für die reine Imagination Ebertys und seiner Nachfolger im 19. Jahrhundert war das fiktive Einhalten eines bestimmten Sehfeldausschnitts bei Vor-, Rück- oder Seitwärtsbewegungen auch in kosmischen Dimensionen kein besonders brennendes Problem. Sinnesphysiologisch und kameratechnisch würde man jedoch mit gewaltigen zoom-Effekten ${ }^{96}$ konfrontiert: Bildwahrnehmung stürzt ins Detail oder verliert sich mit dem Quadrat der Entfernung in Unsichtbarkeit. Auch der seitwärts gerichtete Blick und die von ihm inspizierten hypothetischen Bilderstapel konnten eigentlich bei lichtschnellen interstellaren Beobachterbewegungen nicht unbehelligt bleiben. So oder so: Das von Benjamin beschworene Optisch-Unbewusste hielt mit den verschiedenen Blickattitüden auch markante emotionale Einstellungen bereit

94 Zitiert nach Simonyi, Károly: Kulturgeschichte der Physik, Frankfurt a.M. 1995, S. 410.

95 Einstein, Albert: „Zur Eigendynamik bewegter Körper“, in: Annalen der Physik, 17 (1905); reproduziert in: Lorentz, H. A./Einstein, A./Minkowski, H.: Das Relativitätsprinzip. Eine Sammlung von Abhandlungen [1923], Darmstadt 1974, S. 26.ff.

96 Sehr anschauliche Demonstration in: Morrison, Philip/Morrison, Phylis: Powers of Ten. A Book About the Relative Size of Things in the Universe and the Effect of Adding Another Zero, San Francisco 1982 und Power of Ten, Film von Charles und Ray Eames. 
Für sich genommen musste der seitwärts gerichtete Blick - ähnlich dem rückwärtigen - mit merkwürdiger Selbstbeschränkung bezüglich des Fahrziels behaftet sein. Der eigentliche Fluchtpunkt der Bewegung liegt, wenn man sich an die Stelle von Passagieren versetzt, die nur seitlichen Ausblick haben, am äußersten Rand oder ganz außerhalb des menschlichen Gesichtsfeldes. Der Blick zurück, wenn er alle anderen Blickwendungen ausschließt, war vollends mit resignativer Vertiefung ins Vergangene getränkt. Man braucht sich nur die Aussicht aus dem Endwaggon eines fahrenden Zuges zu Benjamins Zeiten vorzustellen, um dessen ,Engelsyndrom‘, die Reise rückwärts ins Ungewisse, mit großer Intensität nachzuempfinden. - Fazit: Die Weltraumphantasie einer lichtbilderbegleitenden Augenzeugenschaft war immer noch menschlichen Reiseerfahrungen in irdisch-bodennahen Verhältnissen nachgebildet. Nicht einmal die geradewegs vorwärtsschauende Inspektionsbewegung gab vor, in erster Linie auf vorausliegende Ziele (irgendwelche Sterne) fixiert zu sein. Sie sollte vielmehr vornehmlich auf die Archive der ausgestrahlten Erdbilder konzentriert bleiben. Der allumfassende, quasi gleichzeitige, zunächst Gott als jenseitiger Instanz vorbehaltene Überblick wartete nur darauf, von den neuen Demiurgen der technisch erzeugten Bilder, den Filmregisseuren, übernommen zu werden. Auch deswegen hat die von Felix Eberty als visuelles Gedankenexperiment in die Welt gesetzte Imagination so weitverzweigte Wirkungen auslösen können.

\section{Schlussbetrachtung}

Die hier zusammengestellten Proliferationsketten der Ebertyschen Schrift sind nur die Spitze eines Eisbergs, dessen Tiefgang erst noch vollends ausgelotet werden muss. Weitere, hier nicht erwähnte Quellen und Zitierungen deuten darauf hin, dass wesentliche Gehalte oder sogar ausführlichere Passagen in der Schweiz und Frankreich zirkulierten, bevor Flammarion sich den Ideengehalt zu eigen machte. Gegen die bislang vorherrschende Literaturmeinung ist zu betonen, dass Flammarion wohl nur ein intelligenter und phantasievoller, allerdings auch uneingestandener Vervielfältiger der Ebertyschen Weltraumperspektiven gewesen ist auf dem Wege jener lawinenartigen Verbreitung, die schließlich im Berlin der zwanziger Jahre, dem Zentrum filmtheoretischer Debatten, den von Einstein eingeleiteten Neudruck auch unter die Augen Walter Benjamins geführt haben könnte.

Vieles spricht dafür, dass Benjamin - aus welchen Quellen immer zumindest die kursierenden Kerngehalte der Ebertyschen Perspektiven 
übernommen hat: Nicht nur der Engel der Geschichte, der rückwärts in die Zukunft segelt, während ihm die (Bild)Trümmer der Geschichte vor die Füße geschleudert werden und sich zum Himmel türmen, hat das Prinzip der lichtbilderbegleitenden Zeugenschaft Ebertys aufgenommen und ins Tragische gewendet; die ganze Geschichtsauffassung Benjamins ist durchsetzt von bildhaften Aspekten, die sich aus Ebertys Fiktion herleiten lassen. Mit der auratischen Ferne lässt sich ein weiterer wesenhafter Aspekt der Moderne aus Ebertyschen Prospekten entwickeln: Jene Unberührbarkeit der Bilder, die Ludwig Klages im Kosmogonischen Eros sogar am astronomischen Beispiel der in Abwesenheit glänzenden Fixsterne demonstrierte und die Benjamin zur zerschlagungsgefährdeten Kultbildaura transformierte, verband die beiden so gegensätzlichen Persönlichkeiten vor dem Hintergrund einer von der modernen Astronomie ausgelösten, und von Eberty erstmals prinzipiell thematisierten raumzeitlichen Blickpunkteverschiebung in kosmischen Dimensionen; sie endete für Benjamin in der verzweifelten Zuversicht seiner Geschichtsthesen, für Klages war sie der Grundtenor seiner Auffassung vom Geist als Widersacher der Seele. Doch mit den tragisch/antirationalistischen Tonlagen und Tendenzen der Zwischenkriegszeit sind die Folgewirkungen der Ebertyschen Weltraumperspektiven noch keineswegs vollständig erfasst oder vollends abgeschritten. Man kann noch weiter gehen und etwa, wie schon zitiert, in Roland Barthes' Heller Kammer 1980 neuerliche Reflexe dieser revolutionären Einsicht aus der Mitte des 19. Jahrhunderts finden.

Die Gehalte und die erstaunliche Wirkungsgeschichte des EbertyTextes führen letztlich auch zu Problemen einer neuronalen Begründung/Herleitung kunsthistorisch-ästhetischer sowie wissenschaftlichtechnischer Bildgebungsgewohnheiten. Wenn man versucht, Kunst- und Wissenschaftsanschauungen als Hervorbringungen von Kulturprozessen zu betrachten, in denen neuronale und apparative Faktoren mehr und mehr ineinandergriffen, so liefern nicht nur Kunst- und Mediengeschichte, sondern auch vergessene Texte wie der Ebertys überzeugendes Beweismaterial, dass auch das bildliche Denken überformt wurde von neuronal bedingten, gleichwohl historisch gewachsenen, zunehmend instrumentell zugerichteten Visualisierungsprinzipien, die sich über jedes anthropologisch-evolutionsbiologisch zu erwartende Maß hinaus steigerten. Es kam zu regelrechten Erkenntnisumbrüchen. Das Fiktionale und das Imaginäre, ehedem exklusive Domänen von Poetik und reiner Vorstellungskunst, wurden zu Brutstätten einer neuartigen Gattung von wissenschaftlicher Phantasie, die im 20. Jahrhundert höchstes Ansehen 
erlangte: Gedankenexperimente. Deren Werdegang und Bildanteile sind exemplarisch in den hier präsentierten Materialien zu verfolgen.

Das Verhältnis von Technologie, Physiologie und Phantasie im 19. Jahrhundert führt zu einer Geschichte des Blicks als Bildwissenschaft, in der astronomische Fortschritte und Spekulationen eine grundlegende Rolle spielten. Sie lieferten den gemeinsamen Hintergrund für so verschiedenartige Errungenschaften wie Einsteins spezielle Relativitätstheorie, aber auch für die eigenartig melancholischen Rück- und Fernblicke Benjamins und Klages'. Sie halfen zudem, das Konzept eines unablässig laufenden ,kosmischen Kinos', eines von Bildarchiven gefüllten Weltenraums zwischen den Sternen auf den Weg zu bringen. In der phantastischen Verschränkung von technisch/wissenschaftlichem Fortschritt (erste exakte Fixsternentfernungsmessungen 1837-42) und spekulativer Freisetzung von Betrachtern im Weltenraum (zeitgleich mit Edgar Allan Poes Heureka-Schrift, die gegenwärtig unter Kosmologen Furore macht) entwickelte sich ein hochbedeutsamer rezenter Gegenpol zu den Jahrtausende überspannenden Astrologieforschungen, wie sie etwa im WarburgUmkreis betrieben worden sind.

Fazit: Für das Verständnis der Kultur- und Mediengeschichte ist Ebertys Schrift eine Quelle ersten Ranges, die bislang wohl wegen ihrer Anonymität und fächerunspezifischen Abseitigkeit völlig unbeachtet geblieben ist. Es ist ein ungewöhnlicher Glücksfall, dass man so verschiedenartige Ideengebäude wie die Relativitätstheorie, das Konzept eines ,kosmischen Kinos', welches in den zwanziger Jahren so vielfältige Spuren hinterließ, den kosmogonischen Irrationalismus à-la-Klages und Benjamins quereinsichtig aufgesprengte Geschichtsphilosophie derart auf eine einzelne anstoßgebende Schrift zurückführen kann. Wohlbemerkt, Eberty war sicher nicht der einzige, deutlich aus dem Disziplinenkanon ausbrechende Ideenlieferant für besser bekannte geistige Glanzleistungen des 20.Jahrhunderts; aber er hat 1846/47 mit juristisch geschultem Scharfblick aus den physikalisch-astronomischen Einsichten seiner Zeit die ethische Essenz einer wahrhaft universellen Reziprozität möglicher Gesichtspunkte herausdestilliert und so erstmals die Grundstrukturen lichtbildlich-filmischer Fiktionalität entworfen.

Unwiederbringlich Verlorenes betrauernd muss man auch festzuhalten versuchen: Felix Eberty war typischer Vertreter einer hellwachen jüdisch-deutschen Intelligenz, die im besseren Hardenbergschen Preußen ihren geistigen Nährboden hatte. Nicht zufällig hat der Jurist Eberty den Erlös seiner Schrift für die Nothleidenden im schlesischen Riesengebirge bestimmt und sich in einer weiteren anonymen Schrift unter anderem mit 
dem Staatsideal überkonfessioneller Souveränität beschäftigt. Das alles macht ihn nicht nur zu einer zeitgeschichtlich bemerkenswerten Figur, sondern verleiht ihm auch im Hinblick auf gegenwärtige MultikulturDebatten überraschende Aktualität.

Ich bin 2002 durch Zufall oder abseitige Neugier - im Rahmen einer gemeinsam mit Hermann Schweppenhäuser gehaltenen Vorlesungsreihe - auf den Astronomie-Liebhaber Eberty aufmerksam geworden; ich habe alle wesentlichen, zum Teil nur noch in Einzelstücken greifbaren Originalausgaben aufgefunden und zusammengetragen. Der hier nur auszugsweise präsentierte ,bildwissenschaftliche“ Glücksfund bietet viele Ansatzpunkte für eine die Wissenschaftskommunen übergreifende Kulturgeschichte: Von ihm aus können nicht nur die natur- und geisteswissenschaftlichen Zusammenhänge des kosmologischen Gefühlskomplexes der letzten anderthalb Jahrhunderte - astronomische ,Tiefe der Zeit', expandierendes Universum, Urknall etc. - historisch ausgelotet werden. Er bietet auch nachdenkliche Durchblicke auf das sich wandelnde Verhältnis unserer Sprache zu den nunmehr allgegenwärtigen medialen Lichtbildern. Nähe und Ferne sowie die ihnen entsprechenden Tempora: Vergangenheit, Gegenwart und Zukunft haben im ,Visualisierungsersatz' Sprache (immer noch am besten bei Karl Bühler nachzulesen) eine grundlegende Rolle gespielt. Sie haben die Entwicklung der Grammatiken wohl überhaupt erst ermöglicht. Wofür stehen also die Ebertyschen Imaginationen, und was bahnt sich nun an, da mehr und mehr technisch erzeugte Bilder an ihre Stelle treten? - Aber das ist ein weiteres Feld und ein anderes Thema. 


\section{K. LUDWIG PFEIFFER}

\section{ICH LESE, ALSO BIN ICH (NICHT)}

\section{Theoretische Umrisse}

Meine Titelwahl, ich gestehe es vorweg, ist nicht besonders intelligent. Schon die Schlussfolgerung „Ich denke, also bin ich“ zeichnet sich nicht durch die bezwingende Logik aus, die Descartes in ihr erblicken wollte. Valéry nahm daher an, Descartes habe aus seinen geometrischen Talenten die Könnensphantasmen, den Rausch philosophischer Überlegenheit geschöpft; der philosophische Fortschritt verdanke sich dem Vorantreiben von Denkweisen more geometrico in dafür ungeeignete Bereiche. ${ }^{1}$ Der Neurologe Antonio Damasio hält den cartesischen Satz ohnehin für falsch. ${ }^{2}$ Er schreibt dem Körper und den Gefühlen Priorität selbst bei der Entstehung des Bewusstseins zu. ${ }^{3}$ Lawrence Durrell hätte mich - und andere - warnen können. „No, there's nothing wrong with cogito or with sum; it's poor bloody ergo that's been such a curse. ${ }^{\text {"4 }}$ Wie immer: Die Form der Descartes'schen Behauptung hat man für alle möglichen, mehr oder weniger billigen analogen Behauptungen ausgebeutet. In die Serie solcher Ausschlachter und Recycler reihe ich mich nolens volens ein, vorderhand jedenfalls und ohne mich für oder gegen die Klammer zu entscheiden. Ermutigt hat mich immerhin Wolfgang Hildesheimer, der seinem Marbot ein „Pingo, ergo sum“ in den Mund legt und damit, was sachlich schon ernster sein könnte, auf mögliche Spannungen, konstruk-

1 Vgl. Valéry, Paul: „Seconde Vue de Descartes“, in: Euvres, hrsg. v. Jean Hytier, 2 Bde., Paris 1975, S. 843. Vgl. auch meinen Aufsatz „Paul Valéry - Die Vergeblichkeit der Theorie und das okkasionell-theoretisierende Spiel“, in: Pfeiffer, K. Ludwig/Kray, Ralph/Staedtke, Klaus (Hrsg.): Theorie als kulturelles Ereignis, Berlin/New York 2001, S. 286-305.

2 Damasio, Antonio: Descartes' Error. Emotion, Reason, and the Human Brain, New York 1995.

3 Damasio, Antonio: Ich fühle, also bin ich. Die Entschlüsselung des Bewußtseins, München 2000 (Titel der Originalausgabe: The Feeling of what Happens. Body and Emotion in the Making of Consciousness, New York 1999). Vgl. vor allem III. Teil, Kapitel 5.

4 Durrell, Lawrence: Tunc, London 1968, S. 13. 
tive wie problematische, zwischen körperbezogenen performativen und ,rein' mentalen Operationen aufmerksam macht.

Solche Spannungen brechen, wenn man Karl Clausberg folgt, in massiver Form schon dann auf, wenn man sich lediglich intermedialen Text-Bild-Beziehungen, ihrer Wahrnehmung und ihrer ,Lektüre' zuwendet. In der sowohl literaturwissenschaftlichen wie kunstgeschichtlichen Vernachlässigung solcher Beziehungen glaubt Clausberg eine „der seltsamsten systematischen Fehlleistungen der Kulturwissenschaften" namhaft gemacht zu haben: das fast vollständige Übersehen ,jener eigenartigen ausdrucksgeladenen Mischformen, die Texte und Bilder seit der Antike eingegangen sind." Es ist zum Beispiel bestimmt keine triviale Frage, in welchem Sinn und mit welchen Folgen man japanische manga liest oder sieht. ${ }^{5}$

Die Ergebnisse meines Ausschlachtens werden ihrerseits kaum jemanden sonderlich beeindrucken. Es geht, sehr schlicht (mit der Betonung auf dem „,sehr"), um einen Vergleich zwischen dem, was man grob als die Richtungssinne kulturgeschichtlicher und neurobiologischer Perspektiven zum Lesen bezeichnen könnte. Der Vergleich versucht, in zeitlicher Hinsicht, einige der „offenen Spielräume“ (Karl Clausberg, mündlich) auszuloten, die sich zwischen den als Prägungen o.ä. namhaft gemachten langfristigen Vorgaben der Evolutionsbiologie bzw. Hirnforschung und dem hektischen Tagesbetrieb der heutigen Medienszene eröffnen. Theoretische oder auch nur methodologische Ansprüche vermeide ich. Die Neurobiologie, so glaube ich, wird uns keine erkenntnistheoretischen oder ontologischen Umwälzungen bescheren. Sie löst die Probleme nicht, welche die Kultur- und Denkgeschichte über 2.000 Jahre aufgehäuft hat; allenfalls löst sie diese etwas vorschnell auf. Allerdings gestattet sie es, die Zuschreibungsprobleme zwischen kognitiven Opera-

5 Clausberg, Karl „Metamorphosen am laufenden Band. Ein kurzgefaßter Problemaufriß der Sprechblasenentwicklung“, in: Hein, Michael/Hüners, Michael/Michaelsen, Thorsten (Hrsg.): Ästhetik des Comic, Berlin 2002, S. 25. Vgl. die weiteren scharfen Bemerkungen S. 24, S. 26, S. 28, S. 31. Zur interkulturell-intermedialen Stützung solcher Thesen vgl. z.B. Székely, Katalin: Manga und Anime. Japanische Bilderzählungen und ihre Attraktivität für europäische Kulturen, Siegen 2004, S. 12-13 (Diplomarbeit). Zu „manga“ vgl. auch Maeda, Ryozo: „Rasender/Lesender Stillstand. Zur Wahrnehmung von Schrift- und Textbild im Manga", in: Bickenbach, Matthias/Fliethmann, Axel (Hrsg.): Korrespondenzen: Visuelle Kulturen zwischen früher Neuzeit und Gegenwart, Köln 2002. S. 89-107; Maeda, Ryozo: „Bilder lesen, Schriften sehen. Visuelle Kulturen in Europa und Japan“, in: Humboldt kosmos. Mitteilungen der Alexander von Humboldt-Stiftung, 83 (2004), S. 12-13. 
tion, performativen Akten und den möglichen Korrelaten „out there“ begrifflich und methodisch anders zu fassen. Dabei kann es zu Verschiebungen kommen, die mehr oder weniger drastische Revisionen unserer intradisziplinär jeweils gehegten Vorstellungen erzwingen. Selbst bei Exkursionen in die „Welt des Lesens“, 6 die sich weitgehend auf herkömmliche Texte zu beschränken scheinen und insofern den von Clausberg monierten disziplinären Defiziten nicht abhelfen, können sich dann Evidenzen abschatten, die das Nachdenken über zumindest latente (inter)mediale Umbruchssituationen nahelegen.

\section{Kulturgeschichtliche Befunde}

Die kulturgeschichtlichen Befunde, wer würde anderes erwarten, sind uneinheitlich bis widersprüchlich. In seiner unterhaltsamen $A$ History of Reading $^{7}$ zitiert Alberto Manguel seine Mutter, die der jungen Leseratte zuruft: „Go out and live“. Für sie hat das Lesen nichts mit dem zu tun, was sie für das Leben und seine Lebendigkeit hält. Umgekehrt Flaubert, der an Mlle de Chantepie 1857 schreibt: „Read in order to live“ (Motto zum ersten Teil „The Last Page“ in Manguels Buch). Manguel zitiert peronistische Demonstranten, die den Intellektuellen zurufen: „Shoes yes, books no.“ Deren Antwort: „Shoes yes, books yes“ überzeugt niemanden. ${ }^{8}$ Kafka seinerseits empfahl nur solche Bücher zu lesen, die uns beißen und stechen, uns aufwecken wie ein Schlag auf den Kopf. Glücklich wären wir leichter ohne sie. Aber als Axt, mit der wir das gefrorene Meer in uns aufhacken, können wir sie gut gebrauchen. ${ }^{9}$ Mit solcher Metaphorik bewegt sich Kafka auf Versuche zu, das idealiter Bannende, ja Verbindliche ästhetischer Erfahrung zu umschreiben, welches Arnold Gehlen - freilich eher im Blick auf Bilder - mit der Metapher des ,stählernen Nervs" als einer zugleich verengten, überbesetzten und distanzierten Eindringlichkeit zu treffen glaubte. ${ }^{10}$ Man kann auch an den sich auf eine Philosophie der Kunst zubewegenden Schelling denken, für den die Wahrheit aller Erkenntnisse „auf dem Gefühl des Zwangs“ beruht, das sie begleitet. Lässt sich das dem Lesen - irgendwann, irgendwie, gar

6 Vgl. Chartier, Roger/Cavallo, Guglielmo (Hrsg.): Die Welt des Lesens. Von der Schriftrolle zum Bildschirm, Frankfurt a.M./New York/Paris 1999.

7 Manguel, Alberto: A History of Reading, New York 1996, S. 21.

8 Manguel (wie Anm. 7).

9 Manguel (wie Anm. 7), S. 93.

10 Gehlen, Arnold: Zeit-Bilder. Zur Soziologie und Ästhetik der modernen Malerei, Frankfurt a.M./Bonn 1963, S. 11-12. 
systematisch - bescheinigen? Immerhin hat ja Iser seine Theorie ästhetischer Erfahrung im und als Akt des Lesens formuliert. ${ }^{11}$

Wie nähert man sich einem Aktivitätsareal, dessen Zentren zwischen Auge und Gehirn allenfalls durch schlüssig kaum interpretierbare Hirnstromableitungen zugänglich, dessen sozio- wie psychokulturelle Objektivierungsformen extrem uneinheitlich beschaffen sind? Einer Aktivität, die wir einerseits als sowohl elementare wie avancierte unabdingbare, zudem staatlich verordnete Kulturtechnik uns mehr oder weniger contra naturam aneignen müssen, die es uns andererseits zumindest gelegentlich erlaubt, in einen „entrückten, zu Träumereien geneigten und zu assoziativem Denken verführenden Zustand“, in einen „einsamen Rausch“" gleichsam „leidenschaftlich“ zu „versink[en]"? ${ }^{12}$

Selbst diesen Zustand gibt es kulturgeschichtlich offenbar in sehr verschiedenen Versionen. Maxim Gorki liest beharrlich, obwohl er 14 Stunden täglich in einer Bäckerei - einem der Orte, die er ironisch als „meine Universitäten“ bezeichnet - arbeiten muss. Der Schuster, Chartist und öffentliche Vortragsredner Thomas Cooper liest jeden Morgen von drei oder vier bis sieben Uhr, hat auch während der Mahlzeiten ein aufgeschlagenes Buch vor sich und deklamiert Texte bis zur Erschöpfung. Mit 21 Jahren bricht diese radikalisierte Version der "schönen Seele“ in Goethes Wilhelm Meister physisch zusammen und muss für mehrere Monate das Bett hüten. Das sind offenbar, nachdem das 18. schon Phasen der Lesewut kannte, die „neuen Leser im 19. Jahrhundert“. ${ }^{13}$ Eine Art Gegenrechnung macht Claude Lévi-Strauss auf; ihn stimmt der Zusammenhang zwischen Schreib- und Lesepflicht, Militärdienst, Proletarisierung im 19. Jahrhundert melancholisch: „Alle müssen lesen können, damit die Staatsgewalt sagen kann: Unkenntnis des Gesetzes schützt nicht vor Strafe.“14 Das Versinken ins distanzierte und leidenschaftliche, ins entrückte, tagträumende Lesen zum Zwecke „,intellektueller und ästhetischer Befriedigung" sinkt zum "sekundären Ergebnis“ ab, wenn nicht gar nur zum „Mittel“, um das andere, die die „Versklavung“ er-

11 Schelling, Friedrich Wilhelm Joseph: System des transzendentalen Idealismus, hrsg. v. Stefan Dietzsch, Leipzig 1980, S. 71; Iser, Wolfgang: Der Akt des Lesens. Theorie ästhetischer Wirkung, München 1984.

12 Schlaffer, Heinz: „Der Umgang mit Literatur. Diesseits und jenseits der Lektüre“, in: Poetica, 31 (1999), S. 1-25, hier S. 3 u. S. 23.

13 Lyons, Martyn: „Die neuen Leser im 19. Jahrhundert: Frauen, Kinder, Arbeiter", in: Cavallo, Guglielmo/Chartier, Roger (Hrsg.): Die Welt des Lesens: Von der Schriftrolle zum Bildschirm, Frankfurt/New York/Paris 1999, S. 455-497, hier S. 488 u. S. 494-495.

14 Lévi-Strauss, Claude: Traurige Tropen, Frankfurt a.M. 1988, S. 295. 
leichternde Funktion schriftlicher Kommunikation „zu verstärken, zu rechtfertigen oder zu verschleiern“. ${ }^{15}$

Wie verorten wir, zwischen Rausch und Disziplinierung, die vielfältigen Wandlungen, ja die „Leserevolutionen“, von welchen zu sprechen die kulturgeschichtliche Leseforschung sich ermächtigt glaubt? Die erste, so Cavallo und Chartier, findet nicht aufgrund der technischen Revolution des Buchdrucks statt (der über lange Zeit Seitenaufbau, Schriftbild und Aussehen der Handschrift übernimmt), sondern verläuft als zunächst in den Klöstern stattfindende Ablösung mündlicher Praktiken durch den Text als Werkzeug stiller, geistiger Arbeit. Eine zweite Revolution soll mit der Industrialisierung des Buchdrucks im 18. Jahrhundert stattgefunden haben. Man kann sie freilich ganz gegensätzlich als Wende sei es zum extensiven, raschen, sei es zum intensiven Lesen - beide wiederum mit einigen Varianten - beschreiben. Die dritte Revolution werde schließlich durch den Computer markiert, dessen Textverweisungspotenziale „ein ganz und gar originelles und neues Verhältnis zum Text hergestellt" hätten. ${ }^{16}$

Die Situation wird noch beträchtlich komplizierter, wenn man das Lesen genauer, als das die kulturgeschichtliche Leseforschung jedenfalls in ihren anthologieartigen Überblicken tut, mit den medial-materialen Dimensionen der Schreibmaterialien und mit den sehr verschiedenen Schrifttypen der Kulturen (z.B. das Lesen der chinesischen Schrift, nochmals komplexer im japanischen Kontext) in Beziehung setzt - oder bestimmte Einzelereignisse und ihre möglichen Folgen unter die Lupe nimmt Das eine hat vor langer Zeit der oft als Vorläufer von McLuhan gesehene Harold Innis unternommen; zum zweiten hat u.a. André LeroiGourhan vor längerer Zeit Einschlägiges gesagt. ${ }^{17}$ Das dritte, ein Ereignis von beachtlicher Reichweite gerade für das Lesen, speziell für die Entwicklung des wissenschaftlichen Lesens, ja ganzer wissenschaftlicher Disziplinen, könnte man beispielsweise in der Säkularisation von 1802/1803 bzw. in der Zeit von der Aufhebung des Jesuitenordens 1773

15 Lévi-Strauss (wie Anm. 14), S. 294.

16 Cavallo, Guglielmo/Chartier, Roger: „Einleitung“, in: Cavallo, Guglielmo/ Chartier, Roger (Hrsg.): Die Welt des Lesens, S. 45 u. S. 41-44; Wittmann, Reinhard: „Gibt es eine Leserevolution am Ende des 18. Jahrhunderts?“, in: Cavallo, Guglielmo/Chartier, Roger (Hrsg.): Die Welt des Lesens, S. 428439.

17 Innis, Harold A.: Kreuzwege der Kommunikation. Ausgewählte Texte, hrsg. v. Karlheinz Barck, Wien/New York 1997; Leroi-Gourhan, André: Hand und Wort. Die Evolution von Technik, Sprache und Kunst, Frankfurt a.M. 1980, z.B. S. 249-270. 
bis zum Übergang des Regensburger Dalbergstaats an Bayern 1810, vor allem in de damit einhergehenden Auflösung der Klosterbibliotheken erblicken. $^{18}$

\section{Ein längeres Zwischenspiel}

Unabhängig von diesen doch in sehr verschiedene Richtungen zerrenden kulturgeschichtlichen Befunden: Wie soll man einen Begriff vom Lesen bekommen, wenn doch schon sein Gegenstand veschwimmt? Besteht dieser in der Serie der uns geläufigen modern-monotonen Buch-Buchstaben? Oder müssen wir, und wenn ja wann, mit vielschichtigeren visuellen, materiellen, kultischen, und sei es nur noch halluzinierten Elementen, Rändern, Hintergründen, mit einer wie immer verkürzten „culture de l'image“ und „Paralektüren“19 anderstextueller und andersmedialer Art auch dann rechnen, wenn diese sich nicht offenkundig als Illustration, als auratisch-wertvolles Material oder als gebetsartige Nötigung zum „Kauen des Wortes“ präsentieren? ${ }^{20}$ Und wo dergleichen der Lektüre wirklich ausgetrieben worden sein sollte: Müssten wir dann nicht über die Kosten des Lesens und nicht so sehr über seinen Zauber oder Nutzen reden, von der widernatürlich gekrümmten Haltung bis hin zu dem von manchen pauschal konstatierten „Verlust der Sinnlichkeit“" ${ }^{21}$

Mit ziemlicher Sicherheit können wir davon ausgehen, dass der Gegenstand der Lektüre jedenfalls seinem Umfang nach nie hinreichend bestimmbar ist: Die Vorstellung, wohl eher die Illusion, es gehe darum, einen ganzen, ganzheitlichen, „integralen“ Text welchen Umfangs auch immer zu lesen, dürfte im 18. Jahrhundert entstanden und pädagogisch, sodann literaturwissenschaftlich, aber eben kontrafaktisch befestigt wor-

18 Vgl. Kudorfer, Dieter (Hrsg.): Lebendiges BücherErbe [sic]. Säkularisation, Mediatisierung und die Bayerische Staatsbibliothek, München o. J. [20032004], S. 20 (Geisteswissenschaften), S. 51 (Kunstgeschichte), S. 142-143 (Deutsche Philologie und Literaturgeschichte). Für wichtige Hinweise und Hilfestellungen bei der Bewältigung der dem Band zu Grunde liegenden Ausstellung danke ich Edeltraud und Karl Gerlinger, München.

19 Schlaffer (wie Anm. 12), S. 5 und S. 10 dort Fn. 21 in Anlehnung an Gérard Genette und Roger Chartier.

$20 \mathrm{Zu}$ letzterem vgl. etwa Jousse, Marcel: La manducation de la parole, Paris 1975, der zweite Band seiner Anthropologie du geste. Vgl. ferner Gumbrecht, Hans Ulrich/Pfeiffer, K. Ludwig (Hrsg.): Materialität der Kommununikation, Frankfurt a.M. 1995 (vor allem die Beiträge von Werner H. Kelber, Michel Zink und Paul Zumthor).

21 Auch dazu Schlaffer (wie Anm. 12), S. 4 und seine Gewährsleute. 
den sein. ${ }^{22}$ Die Iserschen, schon gegenüber Ingarden verstärkten „Leerstellen“ des Textes und die daraus folgende Potenzialität, der Wirkungsreichtum des Lesens verwandeln sich womöglich in die ,potentielle“, die versäumte, aufgeschobene, vermiedene oder vorgetäuschte, mit symbolischen Vorwegnahmen oder Ersatzhandlungen teilweise oder ganz ersetzte Lektüre. Schlaffer hat sich ironisch über die Techniken ausgelassen, die es angesichts der Unbestimmtheit lesenden Geschehens und seiner Ergebnisse dem Leser, vor allem dem Literaturwissenschaftler erlauben, seine Leseautorität, den Anspruch ganzheitlicher Lektüre auch mit einigermaßen vagen Auskünften über Leseeindrücke zu wahren, ja im Examen erfolgreich den Eindruck zu erwecken, er/sie habe die heterogene Masse der von den Prüflingen vorgeschlagenen und vermutlich gelesenen Texte auch selbst gelesen. ${ }^{23}$ Und weiter: So berechtigt für die Objektebene die Clausbergsche Forderung, man möge doch Text-BildVerhältnisse nicht vernachlässigen, sein dürfte, so wenig klärt sie uns darüber auf, wie solche Kombinationen vom Lesenden/Betrachtenden denn genau verarbeitet werden, wie sich die gedruckten Bild-Text-Welten zu jenen im Bewusstsein flottierenden verhalten.

Kann man gleichwohl das „Panorama des Lesens“ als eines „Neben-, In- und Durcheinander der verschiedenen Art von Lektüren und Nichtlektüren“ ${ }^{24}$ noch anders strukturieren?

Mit der ihm eigenen Treffsicherheit hat zunächst einmal Ralf Schnell dem gegenwärtigen Mediologen, der sich nicht mehr so sehr für die Lektüre als für Krieg und Medien, Militär und Technologie interessiert und sich auf deren Schnittstellen eingerichtet hat, einen Hieb versetzt: Im „Diesseits nackter Technik“ zieht er mit seinen Schülern in die „Zukunft, die seine Gegenwart ist. Und manchmal liest er heimlich ein Gedicht." 25

Ich halte das, vielleicht den Standards der empirischen Literaturwissenschaft nicht ganz genügend, für einen empirischen Befund. Für

22 Schlaffer (wie Anm. 12), S. 1, S. 3, S. 14, S. 17f. u. S. 24 mit entsprechenden Nachweisen. Nur ein weiteres Beispiel: Für das „steinbruchartige“ Leseverfahren selbst eines großen Denkers des Mittelalters wie Wilhelm von Ockham, für die Kenntnisse von „Texten“ aus zweiter und dritter Hand und der vielleicht zentral vom Tageslicht abhängenden Zeit, die man für einen bestimmten Text aufwendet, vgl. Leppin, Volker: Wilhelm von Ockham. Gelehrter, Streiter, Bettelmönch, Darmstadt 2003, S. 36-39.

23 Vgl. Schlaffer (wie Anm. 12), S. 4-7.

24 Schlaffer (wie Anm. 12), S. 23. Schlaffer zählt deren 17. Ich spare mir die ohnehin schon angedeutete Aufzählung.

25 Schnell, Ralf: Orientierung Germanistik. Was sie kann, was sie will, Reinbek bei Hamburg 2000, S. 218. 
empirisch plausibel und relevant halte ich auch die in der freilich paradoxerweise zu stark literarisch ausgerichteten Mediologie des 18. Jahrhunderts von Albrecht Koschorke gezogene weiterreichende These, vornehmlich die Schrift- und Lesekultur, zu Zeiten die entsprechenden Manien des 18. Jahrhunderts hätten jene Affektintensitäten in Form von Intimsemantiken hervorgebracht, die selbst noch in der Emphase der heutigen Militärmediologen und heimlichen Gedichtleser nachhallt. Das stille, einsame Lesen, in welchem die Rhetorik nur einen melancholischen Sonderfall gesehen hatte, mag, in einer Mixtur Foucaultscher und Lévi-Strauss'scher Perspektiven, der Kontrolle und Verwaltung der Phantasie dienen. Aber es beschert der affektiven Intensität, jedenfalls gelegentlich (eben beim heimlichen Lesen von Gedichten), eine attraktive, wenn auch keine allein oder auch nur vornehmlich seligmachende Form. ${ }^{26}$ Dass Koschorke viele, vielleicht wichtigere formgebende Instanzen wie die Oper und damit den in vielerlei Hinsicht, später noch zu besprechenden spezifisch prekären Charakter des Lesens vergisst, ist sein Problem. Wenn man über Leseintensitäten des 18. Jahrhunderts spricht oder schreibt, darf man, wie schon angedeutet, den häufigen nervlichphysischen Kollaps der Lesewütigen nicht vergessen.

Goethe, auf dessen nahezu kollabierende „schöne Seele“ im Wilhelm Meister ich angespielt habe, hegt wie Fichte und viele danach den Verdacht, das Lesevergnügen beruhe auf den relativ simplen Mechanismen der von Geschichten oder sonstigen Texten hervorgerufenen Vorstellungsbilder, deren Klischeehaftigkeit der Leser und vor allem die Leserin nicht durchschaue. Die Klischeehaftigkeit eignet sich zum Beispiel, wie Diderot bei seiner Frau feststellen zu können glaubte, vor allem bei lautem Vorlesen vorzüglich zur Austreibung ehefraulicher Melancholie. Freilich hatten schon die Kirchenväter auch den anderen Fall, das Umschlagen von tagträumerischer Entrücktheit in eben diese Melancholie beklagt, in die Todsünde des Nicht-wirklich-etwas-tun, welches Körper und Seele zerrüttet, in den Zustand einer Verzweiflung ähnlichen acedia versetzt. ${ }^{27}$ Das Lesen, sagt uns die leserorientierte Ästhetik, und die Hirnforschung bestätigt es auf ihre Weise, ist eine Aktivität. Doch hält sich, bis hin zu Heinz Schlaffer, die Überzeugung, es sei doch eine vergleichsweise allzu passive, in keinem relevanten Sinn wirklich performative. Im motorischen Stillstand der Lektüre aber lauert die der Vergeblichkeit dieses Tuns entspringende Unruhe.

26 Koschorke, Albrecht: Körperströme und Schriftverkehr. Mediologie des 18. Jahrhunderts, München 1999, S. 293-298, S. 424f.

27 Manguel (wie Anm. 7), S. 51 u. S. 121. 
Hat sich dieser Verdacht einmal festgesetzt, so verwandelt sich das vermeintlich erregende Buch schnell in langweilig-erdrückende Makulatur, wie bei Lichtenberg, in die „Nullitäten“ der „Bücherfabrikanten“ wie bei Fichte. Kleist berichtet in diesem Sinne über eine Lesebibliothek in Würzburg, wo er keinen Wieland, Goethe und Schiller, dafür aber massenweise Rittergeschichten antrifft; A. W. Schlegel notiert, dem Romaneschreiben vor allem wohne ein trivialisierender Wiederholungszwang inne: „Wie verschieden von der Sprödigkeit des zurückhaltenden Genius, der wie die Löwin nur eins gebiert, aber einen Löwen!“ Ich wüsste eine Reihe von Romanschriftstellern des 20./21. Jahrhunderts, sehr bekannten, zu nennen, welche die Schlegelsche Metapher hätten beherzigen und ihr Glück lieber in einem normalen bürgerlichen Beruf suchen sollen. Kaum einer besitzt die Aufrichtigkeit eines Kingsley Amis, der bereitwillig zugab, kontinuierliches Romaneschreiben sei potboiling, Fließbandproduktion, ganz so wie das Fichte bereits beurteilte und wie es Anthony Trollope im englischen 19. Jahrhundert ganz offen betrieb. ${ }^{28}$ Worin immer das Lesen solcher Texte bestehen mag: ein Kompliment an die Intelligenz der Leser ist es wohl nicht. Trollope druckt einen an ihn gerichteten Brief Thackerays ab, in welchem dieser ihn zur Mitarbeit an einem Magazin auffordert, um aus dem Spinnen von Romanen herausund in die Welt wieder hineinzukommen. Dorothea Veit rät daher Schleiermacher, statt „dreißig Bücher“ von Hardenberg, also immerhin doch Novalis, zu lesen, lieber einmal Tee mit ihm zu trinken, er verstünde ihn dann besser. ${ }^{29}$

Man muss sich daher nicht wundern, dass das entrückte, sich völlig mit erlebten Textdimensionen identifizierende Lesen auch im 19. Jahrhundert und später vorkommt, dass aber seit dem 19. Jahrhundert die andersmediale Redramatisierung des Gedruckten gewaltig ansteigt. Von Dickens ist bekannt, dass und wie er schauspielerartig die Effekte seiner Vortrags- und Romanrezitations-Tourneen vorbereitete. Im Internet (u.a. www.lyrikline.de) kann man heute Lyrikern beim Rezitieren ihrer eige-

28 Vgl. die für die Psychologie des Schreibens und von daher auch für jene des Lesens enorm aufschlußreiche, aus dem Jahr 1883, also einer Hoch- wie Niedergangszeit des Lesens stammende Autobiographie Trollopes von Sadleir, Michael/Page, Frederick (Hrsg.): Anthony Trollope. An Autobiography, Oxford 1980, vor allem S. 52-53, S. 119-121, S. 270-272 u. S. 346-347.

29 Diese und die erwähnten (Lichtenberg, Kleist, Schlegel, Veit) Materialien zur „Lesekultur“ des späten 18. und beginnenden 19. Jahrhunderts in: Richter, Helmut (Hrsg.): Literarische Kultur und gesellschaftliches Leben in Deutschland. Texte und Bilder, Berlin 1988, S. 284-307. Vgl. Trollope (wie Anm. 28), S. 137. 
nen Gedichte zuhören. Neben einem früheren Siegener Studenten, meiner ehemaligen Hilfskraft Marcel Beyer, erlebt man da auch Paul Celans Vortrag seiner „Todesfuge“. Das Ergebnis dürfte oft ein Erlebnis kognitiver Dissonanz sein, wie es bei der bloßen Lektüre in dieser Weise wohl nicht so leicht vorkommt. Und das ist ja schon etwas. Warnt doch auch Gottfried Benn in einem an Hamlets Antwort auf die Frage, was er denn lese: „Words, words, words“ gemahnenden Originalton, „du“ mögest bei den „Worten“ nicht nach der Seele ihres Vor- und Urgesichts fahnden: „Jahre um Jahre - quäle/dich ab, du findest nicht“ („Worte“). Für den intensiv wie extensiv lesenden Literaturwissenschaftler, der mit den Worten so oft „allein“ („Allein: du mit den Worten“, 1. Zeile) ist, klingt das nicht sehr ermutigend.

\section{Aufmerksamkeitspsychologie}

Das schwarz auf weiß Gedruckte kann man also nicht getrost nach Hause tragen, weil es einem auf dem Nachhauseweg zumeist abhanden kommt. Im Bewusstsein verschwimmen die Texte, ,as if its letters were written on water" ${ }^{30}$ Andererseits, so der Neurolinguist André Roch Lecours vom Côte-des-Neiges-Krankenhaus in Montreal, entwickeln sich unsere Gehirnhälften und ihre Sprachpotenziale nicht genügend, wenn wir uns auf den mündlichen Umgang mit der Sprache beschränken. ${ }^{31}$

Wir wissen nicht, wie wir den Begriff des Lesens im Blick auf seine polaren und übergänglichen psychischen Befindlichkeiten und Korrelate, auf Art und Umfang der Texte, ja der Textualität (oder Visualität usw.) selbst, auf das Ausmaß fragmentarischer oder möglichst vollständiger Lektüre hinreichend präzisieren können. Aber wir geraten diesseits der eben einigermaßen launig geschilderten uneinheitlichen kulturgeschichtlichen Evidenzen, angesichts der involvierten Dimensionen der Wahrnehmung, Erinnerung, Wiedererkennung, Erfahrung, Wissen, Übung usw. unweigerlich in die Domänen der Aufmerksamkeitsforschung, Neurolinguistik, der Neurowissenschaften, der Hirnforschung und des jüngst angesagten biokulturellen Ko-Konstruktivismus. ${ }^{32}$ Julian Jaynes hatte in seinem kontroversen Buch The Origin of Consciousness

30 Manguel (wie Anm. 7), S. 65.

31 Manguel (wie Anm. 7), S. 35f.

32 Das zeichnet sich auch bei Manguel (wie Anm. 7), S. 34-47 ab, ohne dass er diese Perspektiven freilich weiterverfolgen würde. 
in the Breakdown of the Bicameral Mind ${ }^{33}$ die These vorgetragen, die ersten Leseerfahrungen, etwa im Medium der Keilschrift, seien noch halluzinierte - Hörerfahrungen gewesen. Nimmt man an, dass solche Neigungen, vielleicht sogar Prägungen nicht gänzlich verschwinden, so könnte dies immerhin das Halluzinatorisch-Lebhafte mancher Leseerfahrungen auch heute erklären helfen.

Gleichwohl klaffen enorme Lücken zwischen den kulturgeschichtlichen Suggestionen und den derzeit führenden (Neuro-)Wissenschaften. Dass diese Lücken nicht zu schließen sind, könnte schon daran liegen, dass zumeist, auch wenn man grundsätzliche Verständnisschwierigkeiten ohnehin in Rechnung stellt, zu schlecht gelesen wird. So schreibt Christian Geyer das Buch Aus Sicht des Gehirns in seiner Rezension konsequent einem mir nicht bekannten Jürgen Roth zu. ${ }^{34}$

Noch besteht freilich Hoffnung, dass sich ein zumindest kontrollierender Rahmen für die weiteren Erörterungen zimmern lässt, wenn man sich an den - mir auch persönlich bekannten - Gerhard Roth und dessen älteres, einiges an Leseanstrengungen erforderndes Buch Das Gehirn und seine Wirklichkeit hält. ${ }^{35}$ Demnach dürfte klar sein, dass Neurobiologie und Hirnforschung einiges auch zum Lesen zu sagen haben, was die gewöhnlich damit befassten geisteswissenschaftlichen Disziplinen als Eingriff, womöglich einschneidenden, in das Repertoire ihrer eigenen Annahmen empfinden müssen. Andererseits können die Neurowissenschaften nichts Endgültiges vermelden, weil nach ihrer eigenen Auskunft die Verdrahtungs- und Funktionsmechanismen des Gehirns zu einem Teil, einem nicht genau bestimmbaren und weitgehend auch vorläufigen Ausmaß, Produkte kultureller und biographischer Dynamiken sind. Ich habe den Eindruck, dass diese Struktur - unvermeidliche, aber immer nur vorläufige, den anderen freilich meist schon zu weit gehende Eingriffe der Neurowissenschaften in die Bestände der Philosophie wie der geisteswissenschaftlichen Einzelwissenschaften - zu einem großen Teil für die relativ fruchtlose Schärfe der Diskussionen zwischen den entsprechenden Repräsentanten verantwortlich ist. Das mag selbst für die kaum

33 Jaynes, Julian: The Origin of Consciousness in the Breakdown of the Bicameral Mind, Princeton 1976.

34 FAZ, 15.9.03. Dieser angebliche Jürgen Roth schreibt nach Auskunft seines Rezensenten im Vorwort die prinzipiell wie im Blick auf das Lesen nicht eben ermutigenden Worte, der Verlag habe ihn gebeten, Aspekte der Hirnforschung und ihrer Bedeutung für ein Menschenbild in einer Weise darzustellen, die ,keine allzu große Geduld und Anstrengung erfordert“.

35 Roth, Gerhard: Das Gehirn und seine Wirklichkeit. Kognitive Neurobiologie und ihre philosophischen Konsequenzen, Frankfurt a.M. 1997. 
abzuweisende These Roths gelten, es gebe nicht den Geist, sondern eine „Vielzahl höchst unterschiedlicher mentaler und psychischer Zustände“ (Erleben von Wahrnehmungsinhalten, Denken, Vorstellen, Aufmerksamkeit, Erinnern, Wollen, Gefühle, der Körperidentität, Selbsterleben, ${ }^{36}$ eine kapitale These, auf die ich zurückkomme.

Wahrscheinlich jedenfalls spalten sich die einzelnen Zustände selbst wieder in eine Vielzahl von Unterzuständen auf. Nehmen wir etwa die für das Lesen zentrale Aufmerksamkeit. Auch wenn man die neuronalen Ursachen für Aufmerksamkeitszuwendung kennt, kann man noch lange nicht die genauen Folgen selbst der elementaren Sakkaden beschreiben, jener drei bis vier mal in der Sekunde erfolgenden Sprünge, mit denen das Auge über eine Seite huscht. Das „Gefühl, als gleite unser Blick gleichförmig über das Geschriebene, ist eine Illusion. “37 Die Psychologie bewilligt uns Aufmerksamkeit zwar als eine „generalisierte zentrale Kapazität“, eine „Art generalisierter mentaler Energie“. Doch vergönnt sie uns diese lediglich als einen nur kurzfristig aufrecht zu erhaltenden „Ausnahmezustand des Organismus“. In ihrem Rahmen muss es einem Leser schwerfallen, seine Aufmerksamkeit durch aufmerksamkeitsfördernde, aber auf oft hunderten von gleichartigen Seiten nur schwer feststellbare Regelbrüche, Pegelsprünge, „pop-out“-Effekte, durch Kriterien der Neuheit, Seltenheit, Unwahrscheinlichkeit, Bedeutsamkeit zu mobilisieren. Dies fällt noch schwerer, wenn man, wie auch die Psychologie gelegentlich, sich der Hirnforschung bedient und die Aktivierung der Aufnahmebereitschaft (,arousal“) eher der rechten Gehirnhälfte zuschreibt. Diese aber ist gerade nicht, oder nicht vornehmlich, für verbal-analytische Operationen zuständig. Der Ausnahmezustand "Aufmerksamkeit“ mag überdies mit einem ,gesteigerten physiologischen Erregungsniveau“" einhergehen, das bei längerem Lesen nur bei entsprechender Spannung gehalten werden kann. Das mag ja immerhin die Popularität von Kriminalromanen und kriminalistischen Strukturen selbst in so genannten normalen, auch postmodernen Romanen erklären helfen. Ansonsten aber legt die Psychologie eine Rangfolge von auditiver

36 Roth (wie Anm. 35), S. 273.

37 Pöppel, Ernst: Grenzen des Bewußtseins. Wie kommen wir zur Zeit, und wie entsteht Wirklichkeit?, Frankfurt a.M./Leipzig 2000, S. 11; vgl. Manguel (wie Anm. 7), S. 37. Ernst Pöppels Sohn David Poeppel treibt derweil Forschungen zum Problem des Hörverstehens weiter: Wie kann man kontinuierliche Inputsignale - die vibrierenden Geräusche im Ohr - verstehen, wenn deren mentale Repräsentation in diskreten Einheiten - Worten vonstatten geht? Vgl. das Programm des Wissenschaftskollegs Berlin vom 21.10.2003, für dessen Überlassung ich Heike Paul danke. 
über visuelle bis hin zu semantisch-verbaler-schriftlicher Aufmerksamkeit nahe. $^{38}$

\section{Die neurowissenschaftliche Rahmung lesender Aufmerksamkeit}

Pöppel hat nun über die in Zehntelsekunden zu messenden Sakkaden und ihr Zerhacken des Lesevorgangs, über seine weiteren allgemeinen Thesen zur Naturwidrigkeit des durch Erziehung und Rechtschreibvorschriften kujonierten Lesens hinaus eine möglicherweise bedeutsame Drei-Sekunden-Front aufgemacht. Er glaubt, dass ein Bewusstseinsinhalt „nur eine Überlebenschance von drei Sekunden hat" und dass es innerhalb der drei Sekunden immer nur einen Bewusstseinsinhalt gibt; ferner, dass diesen Drei-Sekunden-Paketen vor allem in lyrischen Versen und in der Musik - und dies in verschiedenen Sprachen und Kulturen, also auch etwa in der Rezitation und musikalischen Untermalung des japanischen Nô - Gerechtigkeit widerfährt. Längere Verse wie Hexameter, Pentameter und Alexandriner ließen sich in die entsprechenden kleineren Einheiten aufteilen. ${ }^{39}$ Meine E-Mail-Anfrage, wie es unter diesen Voraussetzungen mit der Lektüre längerer Texte bestellt sei, bezeichnete Pöppel als „recht komplex“. Das Drei-Sekunden-Fenster sei eine notwendige, aber keine hinreichende Bedingung ,für die Gestaltung längerfristiger Spannungsbögen" (9.10.2003). Ich selbst vermute, dass hier kulturelles Training und psychisch wirksame, imaginär besetzbare und stark verankerte, unter Umständen sogar (vgl. auch das Beispiel Kriminalroman) mit Tatsächlichkeits- oder Körpervorstellungen attachierte Textbausteine ins Spiel kommen.

Wie immer: Bei aller unaufhebbaren Heterogenität kulturell-medialer Konfigurationen und hirnphysiologischer Befunde - dass Aufmerksamkeit sowohl medial wie auch im Blick auf die Stärke intensiver, an packende Tatsächlichkeits- oder Körperlichkeitsbezüge gemahnende Vorstellungen differenziert werden muss, das legen zunächst einmal auf ihre jeweiligen Arten sowohl die Mediengeschichte wie auch die Hirnforschung nahe. Pöppel verweist auf die Produktivität des Denkens bei moderater körperlicher Aktivität und dessen leichtes Eintrocknen bei

38 Vgl. dazu die viefältigen Beiträge in Neumann, Odmar (Hrsg.): Aufmerksamkeit, Göttingen 1996, vor allem S. 183f., S. 222f., S. 245, S. 336, S. 344 , S. $425 \mathrm{f}$., S. $605 \mathrm{f}$.

39 Pöppel (wie Anm. 37), S. 72 u. S. 85-91. 
(sitzender) Trägheit. ${ }^{40}$ Zwar kann man, so warnt mich Wolf Singer in einer E-Mail, ${ }^{41}$ längeres intensives Lesen nicht als sensorische Deprivation, nicht einmal als deren Vorform ansehen. Ich hatte gehofft bzw. befürchtet, man könne dies, um auf diese Weise die intensiv-halluzinatorische Qualität mancher, auch langtextbezogener Leseerlebnisse zu erklären. Denn, darauf beharrt vor allem Lurija, bei sensorischer Deprivation treten leicht Halluzinationen auf. ${ }^{42}$ Singer legt mir nahe, Lurijas Betonung der visuellen Basis auch von Gegenstandsbezeichnung, Begriffsbildungs- und per implicationem von Leseprozessen, das Betreiben von Reflexion auch und vielleicht vor allem als Abrufen visueller Gedächtnisspuren nicht $\mathrm{zu}$ verallgemeinern. ${ }^{43}$ Visualisierungsfähigkeiten (und -nötigungen) seien bei verschiedenen Menschen sehr unterschiedlich ausgeprägt.

Aber die meisten Hirnforscher bringen gleichwohl starke Evidenzen dafür bei, dass entscheidende Bewusstseins-, vielleicht auch, in Pöppelscher Terminologie: Außer-, Neben-, Unterbewusstseinsprozesse der Sprache und dem Lesen vorausliegen. Singer selbst hält nichtverbale, „nichtrationale“ Kommunikationsformen - er nennt meditative Verfahren, bildende Kunst, Musik, Tanz, Medien, in denen sich nichtverbale Inhalte speichern und multiplizieren lassen und kann sich deren Praxis sogar auf Konferenzen vorstellen - für „,natürlicher“ als verbal-rationale. ${ }^{44}$

Auseinander halten muss man wohl vor allem sprachliches und musikalisches Hören und, a fortiori, musikalisches Hören und sprachliches Lesen. Auf „peripherer Ebene“ werden sie gemeinsam verarbeitet, „,dann trennen sich aber die Wege". 45

Natürlich heißt dies nicht, dass sich die - vor allem jungen - Leute noch mehr vom Sound irgendeiner Rockband behämmern lassen, noch mehr die überbordenden, vielbeklagten Bilderfluten reinziehen sollen. Karl Clausberg hat zu Recht notiert: „Die eigentlichen Orte unserer Bil-

40 Pöppel (wie Anm. 37), S. 184.

41 E-Mail von Wolf Singer (24.9.2003).

$42 \mathrm{Vgl}$. Lurija, Alexander R.: Das Gehirn in Aktion. Einführung in die Neuropsychologie, Reinbek bei Hamburg 2001, S. 51.

43 Lurija (wie Anm. 42), S. 28, S. 160, S. 217, S. 239 u. S. 320. Vgl. auch ders.: Romantische Wissenschaft. Forschungen im Grenzbezirk von Seele und Gehirn, Reinbek bei Hamburg 1993, S. 82; S. 84-87 zur Forschung über Analphabeten.

44 Singer, Wolf: Ein neues Menschenbild. Gespräche über Hirnforschung, Frankfurt a.M. 2003, S. 79, S. 92f. u. S. 103 und ders.: Der Beobachter im Gehirn. Essays zur Hirnforschung, Frankfurt a.M. 2002, S. 222 u. S. 233f. sowie die genannte E-Mail (wie Anm. 41).

45 Singer (wie Anm. 41); vgl. Lurija (wie Anm. 42), S. 37. 
der liegen in uns selbst; aber die Macht der medialen Verführungen und Verrücktheiten lässt sie immer weiter zurücktreten.“ ${ }^{46}$

Man kann also zwar mit Olaf Breidbach einen Text als „Gefüge von Zeichen" bezeichnen, bei dessen Digitalisierung es keine Rolle spielt, ob es sich um „Pixel eines Bildes, digitalisierte Teilbereiche einer Messkurve oder numerisch kodierte Buchstaben eines Textes" handelt. Die Maschine kennt keine Buchstaben, keine Bilder. Aber das interpretierende Bewusstsein unterscheidet da sehr wohl. Daher erscheint es mir fraglich, ob man den Begriff des Bildes zur „Aus-Bildung des in den Sensoren Erfahrenen" verallgemeinern kann. Wenn bei dieser Aus-Bildung dem visuellen Bild gleichwohl ,eine besondere Bedeutung zukommt", dann möchte man gerne wissen, worin sie besteht und wie die Aus-Bildung als Justierung von Bildwahrnehmung und Textlektüre aussehen könnte. Ich weiß nach der Lektüre von Breidbach jedenfalls nicht, wie ich mir die revitalisierte Schellingsche ,intellektuelle Anschauung“ als eine „Zuordnung von Denken und Anschauen“, eine enge Verzahnung von „Bild-Wahrnehmungen und Sprache“ vorstellen soll. ${ }^{47}$

\section{Neurowissenschaftliche Konturen polymodalisierten Lesens}

In einem letzten Abschnitt will ich das Thema Bewusstsein und mediale Differenzierung nochmals variieren. Manchmal, so will es nicht nur bei Breidbach scheinen, erschrickt die Hirnforschung davor, dass ihre eigenen Ergebnisse sie zu weit in die Arme von Irrationalismen und Reflexionsarmut treiben. Sprach- und reflexionsbewusste Philosophen wie Habermas halten ihr das immer wieder mal vor. Vielleicht versuchen das philosophiebewusste Hirnforscher auch dadurch abzuwehren, dass sie,

46 Clausberg, Karl: „Video, ergo sum? Licht und Sicht in Descartes' Selbstverständnis sowie Fludds Erinnerungsscheinwerfer. - Ein Ausblick auf die Kunstgeschichte der virtuellen Bilder zwischen Mnemonik und Projektionstechnik“, in: Breidbach, Olaf/Clausberg, Karl (Hrsg.): Video Ergo Sum. Repräsentation nach innen und außen zwischen Kunst- und Neurowissenschaften, Hamburg 1999, S. 8-33 u. S. 32.

47 Breidbach, Olaf: Das Anschauliche oder über die Anschaunng von Welt. Ein Beitrag zur Neuronalen Ästhetik, Wien/New York 2000, S. 2-3, S. 16, S. 95 u. S. 105-106. Die historischen Evidenzen früherer Hirnforschung sehen daher doch oft etwas anders aus. Vgl. auch ders: Die Materialisierung des Ichs. Zur Geschichte der Hirnforschung im 19. und 20. Jahrhundert, Frankfurt a.M. 1997, S. 214-215 auch zu Leonardo; S. 352 zu Lashleys Ich-Begriff als "stereotype of memories centering about the body image“, S. 372-373. 
wie Gerhard Roth, Kognitive Neurobiologie als jenen Teil der Neurobiologie definieren, der sich mit den „neurobiologischen Grundlagen kognitiver, bedeutungshafter Leistungen “48 beschäftigt. In diesem Fall aber müsste eine Binnendifferenzierung des „Bedeutungshaften“ zumindest dann erfolgen, wenn dazu etwa „multisensorische“ Erkennungsprozesse zählen sollen. Natürlich ist alles irgendwie bedeutungshaft. Roth hält sich aber sehr, für mein Gefühl zu sehr bei der Frage zurück, ob nun „Geist und Bewusstsein“" an die Existenz von Sprache gebunden sind, ${ }^{49}$ was wie von Bewusstsein begleitet ist. ${ }^{50}$

Man kann kulturgeschichtliche und diskurstheoretische Gründe dafür angeben, dass im Laufe der Zeit die bedeutungsverstehende Lektüre von verschiedenen Textsorten durch Auslegungsvorschriften (,Hermeneutiken') mehr oder weniger (eher weniger) erfolgreich geregelt und u.a. dadurch die Trennung von Bildwahrnehmung und Textlektüre besiegelt wurde. Die Bedeutungs- und Auslegungskontrolle begann mit zunehmend komplexen, bildlich nicht mehr illustrierbaren theologischen Texten. Sie setzte sich über juristische und historische Textsorten fort und endete im 19. Jahrhundert (vorläufig) mit der Leseregulierung auch der literarischen. Dass diesen Anstrengungen kein allzu großer Erfolg beschieden war, liegt nicht nur an der Produktivität dekontextualisierter Schriftlichkeit: Jeder Satz ruft andere, von ihm zunächst abgeblendeten Formulierungs- und Verstehensmöglichkeiten auf. Es liegt auch daran, dass selbst das Erkennen nach Lurija immer ",polymodal“ verfährt, ${ }^{51}$ dass - darin sind sich die Hirnforscher wohl einig - „Geist“ aus einer Vielzahl psychischer und mentaler Zustände besteht.

Dann aber wird man sich, bei allen Risiken, an die Differenzierung der Polymodalität machen müssen. Dann geht es zumindest nicht mehr an, wie Gilles Fauconnier und Mark Turner einen einheitlichen, auf „conceptual blending" beruhenden Denkbegriff, ein zwar nicht in seinen bedeutungshaften Inhalten, aber doch seinen literarisch-sprachlichen Verfahrensmodaliltäten angeblich homogenisiertes Bewusstsein zu behaupten. Fauconnier, immerhin Chairman des Cognitive Science Department at the University of California, San Diego, und Turner, der vom Professor of English zum Associate Director of the Center for the Advanced Study in the Behavioral Sciences at Stanford University mutierte,

48 Pöppel (wie Anm. 37), S. 31, m. H.

49 Pöppel (wie Anm. 37), S. 218.

50 Pöppel (wie Anm. 37), S. 219f., vgl. auch S. 307 zu den in Anführungszeichen gesetzten „Tiefen“ unserer „unbewußten“ Gedächtnisinhalte, in denen die ,eigentlichen Antriebe unseres Verhaltens" liegen.

51 Pöppel (wie Anm. 37), S. 70. 
behaupten allen Ernstes, es liege ein originärer Denkprozess, also ein Fall von „conceptual blending“, wie die Zauberformel lautet, im Falle des Skilehrers vor, der seinen Zöglingen eine Skibewegung mit der Instruktion beizubringen versucht, sie sollten sich einen ein Tablett mit einem Champagnerglass und Croissants mit aller Vorsicht tragenden Kellner in einem Pariser Café vorstellen. Natürlich sind hier Elemente von „conceptual blending“ im Spiel. Aber das Entscheidende dürfte doch, worauf etwa Moshé Feldenkrais immer wieder hingewiesen hat, wohl die Bewusstheit eines bildlich vorgestellten Bewegungsablaufs und des Bewegungsgefühls dabei sein. Fauconnier und Turner räumen das en passant auch ein: „What counts are direction of gaze, positioning of the body, and overall motion. ${ }^{\text {“52 }}$

In seinem vorhergehenden Buch The Literary Mind. The Origins of Thought and Language ${ }^{53}$ hatte Turner gar „,conceptual blending“, Alltagsdenken und literarische Schemata zur großen Einheitssauce verrührt. ${ }^{54}$ In der Tat: Wer wollte bestreiten, dass wir andauernd mit Geschichten, Projektionen, Parabeln (wobei „parable“ „the projection of story“ is ${ }^{55}$ ) - kurzum: ,narrative imagining“ als „fundamental instrument of thought ${ }^{\star 56}$ - operieren? Vorübergehend schieben sich die Themen ,image schemas“, „complex image schemas“, „kinesthetic“ oder „auditory“ oder „tactile image schemas“ in den Vordergrund. Deren Integration habe Antonio Damasio mit dem Begriff der „convergence zones" versucht. Damasio bemühe sich, Ordnung in die Vielfalt - und die vielfältige Hirnlokalisierung - des Gedächtnisses, der motorischen und der bedeutungshaften Prozesse zu bringen.$^{57}$ Die Theorie Edelmans über neuronale Gruppenselektion wird hinzuzitiert. Doch dann macht Turner fröhlich mit heruntertransformierten Metaphorologien weiter. Lakoffs und Johnsons Metaphors We Live By wird ins Feld geführt, ${ }^{58}$ zumeist abgesunkene, zu Geschichtchen und Geschichten montierte Elementarmetaphern (,Die Kommission gab schließlich nach und brach zusam-

52 Fauconnier, Gilles/Turner, Mark: The Way We think. Conceptual Blending and the Mind's Hidden Complexities, New York 2002, S. 21. Typisch die Totalansprüche, etwa „Part Two: How Conceptual Blending Makes Human Beings What They Are, For Better and for Worse" mit Unterkapiteln von "The Origin of Language" bis hin zu „The Way We Live“.

53 Turner, Mark: The Literary Mind. The Origins of Thought and Language, New York/Oxford 1996.

54 Turner (wie Anm. 52), S. 115.

55 Turner (wie Anm. 52), S. 7.

56 Turner (wie Anm. 52), S. 4.

57 Turner (wie Anm. 52), S. 22f. mit Hinweis auf einen Aufsatz Damasios.

58 Turner (wie Anm. 52), S. 39. 
men") werden angeführt, die wir und die uns so manche Literatur im Sinne W. Schapps für unsere Verstrickungen des Lebens tagtäglich zusammenbrauen. ${ }^{59}$

Mit Turners „literary mind“ kommen wir wohl nicht weiter. Vermutlich aber mit den von ihm und Fauconnier so schlampig zitierten und halbherzig verwendeten Hirnforschern Edelman und Damasio. Edelman und Tononi haben in einem neueren Werk $A$ Universe of Consciousness. How Matter Becomes Imagination ${ }^{60}$ ähnlich wie auch Roth vor allem die nach Inhalten, Intensitätsgraden und Bewusstseins-/Bewusstheitsabstufungen, nach Schattierungen, Tönungen und Aufmerksamkeitsgraden variierende ,incomparable richness of being “61 hervorgehoben: „variability“, „the variety of discriminable situations “62 darf als Schlüsselwort gelten. Die Integration dieser nach Milliarden zählenden möglichen Bewusstseinssituationen ist weitaus schwieriger als sich das Turner vorstellt. Es besteht Einigkeit bei den ,seriösen“ Hirnforschern darüber, dass weder Bewusstsein überhaupt noch auch etwa Gedächtnisleistungen wie ein Computer organisiert sind. Beide lassen sich nicht in Repräsentationsbegriffen beschreiben, wie wir sie bei den ,symbolischen' Aktivitäten unserer syntaktischen und semantischen Fertigkeiten verwenden. Denn die Bewusstseinssignale enthalten keine präcodierten Botschaften; wir kennen die Bewertungscodes des Gehirns, vor allem des limbischen Systems nicht, die für die Qualia (Damasio), das "selective matching“ verantwortlich sind. ${ }^{63}$ Einheit und Kohärenz solcher Mannigfaltigkeit sind durch enge Kapazitätsgrenzen beschränkt - ,we cannot keep in mind more than a few things“", es gibt nur wenige ,independent entities“, die wir in einem bewussten Zustand unterscheiden können, ohne dass „the integration and coherence of that state" gefährdet würde. ${ }^{64}$

Im Blick auf die geforderten Integrationsleistungen des Lesens vor allem bei längeren Texten stimmt das nicht gerade hoffnungsfroh. Generell kann das durchs Gehirn vermittelte „phenomenal experience“65 sprachlich schlecht wiedergegeben werden. Edelman und Tononi wollen deshalb nicht zu den Mystikern überlaufen. ${ }^{66}$ Sie machen Hoffnung, dass

59 Vgl. z.B. Turner (wie Anm. 52), S. 29 u. 38-56.

60 Edelman, Gerald M./Tononi, Giulio: A Universe of Consciousness. How Matter Becomes Imagination New York 2000.

61 Edelman/Tononi (wie Anm. 59), S. 29, vgl. auch S. 20-22.

62 Edelman/Tononi (wie Anm. 59), S. 31, S. 32, S. 85.

63 Edelman/Tononi (wie Anm. 59), S. 94f.

64 Edelman/Tononi (wie Anm. 59), S. 26.

65 Edelman/Tononi (wie Anm. 59), S. 220.

66 Edelman/Tononi (wie Anm. 59), S. 220 u. S. 222. 
es sowohl wissenschaftlich wie auch medial erheblich $\mathrm{zu}$ differenzierende Umwege der Annäherung gibt. Aber welche?

Der medialen Differenzierung hat sich am entschiedensten wohl Damasio angenommen. Für ihn ist das menschliche „Kernbewusstsein“ nichtsprachlich; der repräsentationale, der Bezeichnungscharakter der Sprache spricht gerade dagegen, dass sie für das Bewusstsein viel bedeutet, denn offenbar geht den Bezeichnungen ja etwas voraus, was durch die Sprache dargestellt, vor allem aber auch fiktionalisiert wird. ${ }^{67}$ Die Erzeugung von Bewusstseinskarten kann man sich besser als Film oder auch als Pantomime vorstellen - so wie etwa Jean-Louis Barrault in Kinder des Olymp einen Uhrendiebstahl mimisch darstellt. ${ }^{68}$ „Wortloses Geschichtenerzählen ist natürlich“. ${ }^{9}$ Es geht der Sprache, deren Vorbedingung es ist, voraus - und fällt nicht wie bei Turner damit zusammen. ${ }^{70}$ Gerade Hamlet zeige mit seinen elementaren Fragen (,Wer da?" usw.), so Damasio in einer für Shakespeare-Experten in der Tat interessanten These, wie schwierig es sein kann, die Ursprünge und die Koordinaten der eigenen Situation zu begreifen. ${ }^{71}$ Selbst das erweiterte Bewusstsein, welches so etwas wie autobiographische Strukturierungsmomente (elementare Erfahrungsgeschichten wie einen zeitlich gegliederten Vorstellungsraum von Schmerz und Freude) enthält, kommt ohne Sprache aus, nicht aber ohne beträchtliche Gedächtnis- und Denkleistungen. ${ }^{72}$ Es wird durch Sprache bereichert, aber eben auch fiktionalisiert. ${ }^{73}$

Wer liest, begibt sich daher nicht nur in der Literatur zumindest tendenziell in ein Feld von Fiktionen. Diese neigen dazu, polymodale Intensitäten des Bewusstseins zu zersetzen. Daher bilden fast alle Textsorten Strategien aus, welche dieser Zersetzung gegensteuern. Solche Strategien können bei vielen nichtliterarischen Textsorten im gezielten Einsatz von plausibilisierbaren Normen und Interessen bestehen, die sich mit Beispielen erläutern, ,illustrieren“ lassen (müssen). Im Blick auf literarische Texte freilich könnte ein unfreiwillig ironischer Kurzschluss in der Feier von Fiktionalität stecken, welche die Literaturwissenschaft oft der Literatur hat zuteil werden lassen. Erfahrene, teilweise auch andersmedial tätige Autoren wie Samuel Johnson, Oscar Wilde, Wolfgang Hildesheimer u.a. haben bemerkt, dass diese Art der Fiktion weder eine

67 Damasio (wie Anm. 3), S. $225 \mathrm{f}$.

68 Damasio (wie Anm. 3), S. 224 u. S. 375f.

69 Damasio (wie Anm. 3), S. 228.

70 Damasio (wie Anm. 3), S. 229.

71 Damasio (wie Anm. 3), S. 229.

72 Damasio (wie Anm. 3), S. 237.

73 Damasio (wie Anm. 3), S. 29. 
Art Anbiedern der Literatur bei den gesellschaftlich herrschenden und deshalb für Realität gehaltenen Fiktionen verhindert (Oscar Wilde nannte das ,the decay of lying“) noch vor einem quälenden Irrelevanzverdacht schützt. Samuel Johnson wunderte sich daher gelegentlich über Leute, die literarische Bücher zu Ende lasen. Spätestens seit Oscar Wilde steht die Frage, aus welchen Texten eine Literaturgeschichte bestehen könnte, einigermaßen offen im theoretischen Raum. Man kann die Frage heute umformulieren: Wie gelingt es den - metaphorischen usw., textuell ohnehin etwas blassen, in der Moderne nochmals vielfältig gebrochenen Konkretisierungs- und Verlebendigungsstrategien der Literatur, in das polymodale Spiel der derzeit neurowissenschaftlich erhärteten Tatsächlichkeiten des Bewusstseins und des Imaginären einzusteigen?

Sicher lassen sich, wenn man nicht wieder einmal irreführende Banalitäten wie die vom Tod der Literatur oder auch ihre hochgemute Apologie verkünden will, gute Antworten auf solche Fragen finden. Sicher dürfte aber auch sein, dass für die Zwecke des polymodalen Spiels neben der etwa vom Film verkörperten intermedialen Typik die emotional-motorische Kopplungsfähigkeit der Musik und ihr Assoziationspotenzial eine zentrale Rolle spielen (vgl. auch den Beitrag Altenmüller in diesem Band). Auch hier aber müssten die Kulturwissenschaften und die in deren Rahmen, nicht in ihrer heute meist üblichen pseudoverselbständigten Form agierenden Medienwissenschaften die Codierungsweisen solcher Kopplungen (beispielsweise nach Instrumentalmusik und Musik, in denen auch die menschliche Stimme eine Rolle spielt) präzisieren. Dieser - auch anthropologisch zu nennenden Aufgabe - hat sich die Musikwissenschaft nicht zureichend angenommen.

Das heißt nun alles nicht, dass man nicht die Steigerung, sondern die weitere Absenkung sprachlicher Leistungen, das noch reflexionslosere Eintauchen in die Ströme der allerorten zum Billigtarif angebotenen Bilder oder Töne fordern könnte.

Was aber folgt dann aus den vorstehenden Ausführungen, wenn überhaupt etwas daraus folgt? Ich riskiere einige institutions- bzw. disziplinbezogene Andeutungen. Da man die Lesekompetenz nicht reduzieren darf, sondern eher noch steigern sollte, müssten die Selektionen im Blick auf Leseinhalte sowohl härter wie auch variationsreicher werden. Die Lektüre eines Kanons, einer vermeintlichen kohärenten geschichtlich ausdifferenzierten Serie von Texten (,Literaturgeschichte') kann nicht eingeklagt werden. Stattdessen müsste die Lektüre bestimmter Texte sowohl nach Umfang wie Intensität und Interesse von Fall zu Fall begründet werden. Dabei kann man sich Ablehnungen einhandeln. Jedenfalls 
drängt sich mir der Eindruck auf, dass die Einrichtung der Literaturwissenschaften als unabhängiger Fächer (mit, selbstverständlich, ewigen Außenbegründungsproblemen sowohl kultureller wie theoretischer Art) insoweit ein akademischer Irrläufer war, als diese sich über lange Zeit als flächendeckende Textinterpretationsanstalten verstanden. Dilthey hatte sicher ursprünglich etwas anderes, kulturwissenschaftlich Weiteres im Blick, auch wenn sich dieser Blick zeitweilig auf das „Erlebnis und die Dichtung“ verengt haben mag. Selbst die verdienstvollen und anstrengenden Bemühungen, kritisch-historische Texte und Gesamtausgaben von Autoren zu erstellen, verstehen sich beileibe nicht von selbst. Man muss nicht das Beispiel des Herder-Herausgebers Suphan bemühen (der sich nach getaner Arbeit auf einige Bände seiner Edition stellte und erhängte). ${ }^{74}$ Man muss auch nicht Oskar Becker folgen, der da in einem Aufsatz mit dem ,schönen“ Titel „Von der Hinfälligkeit des Schönen und der Abenteuerlichkeit des Künstlers. Eine ontologische Untersuchung im ästhetischen Phänomenbereich“ meinte, an den gesammelten Werken rätselten die Epigonen - und auch der Künstler selber - herum, die gesammelten Werke lägen außerhalb der genuin ästhetischen Sphäre. ${ }^{75}$ Es genügt, an Shakespeare zu denken. Es könnte sein, dass ein ,authentischer Shakespeare" gerade nicht in der Fixierbarkeit des Textes, sondern in der ständigen performanztauglichen Neu- und Umschreibung vorläufiger Textbausteine besteht. ${ }^{76}$

Die Neurobiologie, speziell die Hirnforschung, dürfte den Kulturund Medienwissenschaften daher am ehesten die Erforschung polymodaler Verhältnisse nahelegen. Kultur- und medienwissenschaftlich schla-

74 Heinz Schlaffer bringt das Beispiel in seinem Buch Poesie und Wissen. Die Entstehung des ästhetischen Bewußtseins und der philologischen Erkenntnis, Frankfurt a.M. 1990, S. 224.

75 Becker, Oskar: „Von der Hinfälligkeit des Schönen und der Abenteuerlichkeit des Künstlers. Eine ontologische Untersuchung im ästhetischen Phänomenbereich“, in: Festschrift E. Husserl zum 70. Geburtstag gewidmet. Ergänzungsband zum Jahrbuch für Philosophie und phänomenologische Forschung, Tübingen 1974, S. 27-52. Becker operiert übrigens schon mit dem heute neurowissenschaftlich durchaus verwendbaren englischen Begriff des ,thrill“, S. 34f.

76 Dazu Orgel, Stephen: The Authentic Shakespeare and Other Problems of the Early Modern Stage, New York 2002. Vgl. vor allem das Kapitel „What is a Text?“. Vielleicht sollte ich nichtanglistische Leser darauf hinweisen, dass Orgel einer der angesehensten historisch-gattungstheoretisch arbeitenden Forscher im Blick auf die englische Renaissance, speziell die Maskenspiele ist. $\mathrm{Zu}$ diskutieren wären hier auch eine Reihe von Büchern von Robert Weimann, der die Macht und Integrität auch des Shakespeareschen Textes höher als Orgel veranschlagt. 
gen sich diese als Medienkonfigurationen nieder. Die Geschichtlichkeit der Wahrnehmung könnten wir neurobiologisch als Umbesetzungen in den Figuren der Polymodalität, diese medienwissenschaftlich ab und an als Medienumbrüche zu erfassen versuchen.

Wären wir ein Genie wie Shakespeare, so Damasio, ${ }^{77}$ könnten wir die Kämpfe unseres Selbst dazu benutzen, das ganze Personal des abendländischen Theaters oder was auch immer zu erschaffen. Wären wir Fernando Pessoa, könnten wir die Komplexität vier verschiedener Dichter mit einer Feder bzw. einem Computer entwerfen. Aber am Ende ist es, nach kaum 20 Jahren Theaterlaufbahn, derselbe Shakespeare, der sich nach Stratford zurückzieht und seiner Frau sein zweitbestes Bett vermacht, derselbe Pessoa, der die leseerzeugte Komplexität in einem Lissaboner Krankenhaus im Alkohol zu vergessen sucht. Wir könnten auch an Rimbaud denken, der als Teenager ,große', aber eigentlich nicht mehr zu interpretierende Gedichte schreibt und Erhebliches an Drogen konsumiert, danach sich zum erfolgreichen Geschäftsmann zwischen Afrika und Europa mausert und nach einer Knieoperation mit 36 Jahren stirbt. An die „tragische Literaturgeschichte“ Walter Muschgs wäre zu erinnern. $^{78}$

Der viel schreibende und sehr schwer zu lesende A. N. Whitehead - der gelegentlich Briefe Bertrand Russells nicht beantwortete, weil sie ihn von mathematischer Denkarbeit abhielten, die europäische philosophische Tradition für eine Folge von Fußnoten zu Plato hielt, der sich für die Rolle der Eingeweide und körperlicher Gefühle ebenso wie für die Möglichkeit von ,presentational immediacy“ interessierte - dieser Whitehead sah die Grenzen der lesbaren Welt eng gezogen. „The philosophy of organism abolishes the detached mind“" ${ }^{79}$ - ein distanziertes Bewusstsein, welches die Formen eines interpretierend geregelten, aber auch noch jene des wirkungsästhetisch in die Freiheit entlassenen Lesens eigentlich voraussetzen. Die Selbstbestimmung seiner Philosophie als realistische Version des absoluten Idealismus F. H. Bradleys, die ebenso umfassende wie differenzierte Theorie der Gefühle als positiver Erfassungsakte im Rahmen einer Zell-Theorie des Wirklichen (actuality), - all das ließe sich womöglich heute annäherungsweise neurowissenschaftlich reformulieren und kunst- bzw. medienwissenschaftlich differenzieren. Vielleicht könnten sich diese Disziplinen in entsprechend stilistisch-be-

77 Vgl. Damasio (wie Anm. 3), S. 272.

78 Muschg, Walter: Tragische Literaturgeschichte, Bern/München 1969.

79 Vgl. Whitehead, A. N.: Process and Reality. An Essay in Cosmology, New York/London 1978, S. 56. 
grifflicher Ernüchterung dann an Whiteheads Bestimmung der Philosophie zumindest anhängen:

Philosophy is the welding of imagination and common sense into a restraint upon specialists, and also an enlargement of their imaginations. By providing the generic notions philosophy should make it easier to conceive the infinite variety of specific instances which rest unrealized in the womb of nature. ${ }^{80}$

Mit dieser ,Definition“ der Philosophie liefert Whitehead natürlich keine Bestimmung des Lesens. Wollte man sie gleichwohl als eine Art Inspiration für eine solche Bestimmung benutzen, müßte man sich Analogien und Differenzen ausdenken. Ein Set solcher Analogien und Differenzen bestünde sicherlich im instrumentellen Charakter (,By providing the generic notions philosophy should make it easier...") der jeweiligen Tätigkeit. Das polymodale, die Übergänge in Anderes vorbereitende und organisierende Lesen wäre dann ein notwendiger (Um)Weg zu seiner eigenen, immer wieder aufgeschobenen Abschaffung.

80 Vgl. Whitehead (wie Anm. 79), S. 17 u. S. xiii (mit einer Liste der von Whitehead verschmähten Denkgewohnheiten), S. 11-13 (Sprachkritik, vgl. S. 48-51), S. 17 u. S. 39 (Fußnoten zu Plato), S. 122 (Eingeweide, ,presentational immediacy“ gerade bei „delusions“), S. 162-163 u. S. 219-280 (Theorie der Gefühle). 

PETER M. HeJL

\title{
MEDIENWISSENSCHAFTEN UND WAHRNEHMUNGSBIOLOGIE.
}

\author{
ZUM PROBLEM EINER NICHT-BEZIEHUNG ${ }^{1}$
}

\section{Einleitung}

Sicher nimmt jedermann in einem gewissen Maße an zu wissen, wie Wahrnehmen und Handeln funktionieren. In diesem Sinne gab und gibt es natürlich seit jeher auch Beziehungen zwischen „den Medienwissenschaften" und „der Wahrnehmungsbiologie“. Wir orientieren uns alle in vielerlei Zusammenhängen mit Hilfe von Alltagsvorstellungen, die auch Annahmen darüber enthalten, wie wir wahrnehmen. Solche Annahmen und Überzeugungen sind teilweise sogar zu Netzwerken verbunden, so dass man von „Alltagstheorien“ oder von implizitem Wissen ${ }^{2}$ spricht.

In den letzten Jahrzehnten sind überdies eine Reihe von Kenntnissen und Fragestellungen aus verschiedenen Disziplinen, die an Wahrnehmungsproblemen arbeiten, in andere Wissenschaftsbereiche übernommen worden. Freilich scheint es angemessen zu sein, diese Übernahmen mit der Metaphorik der „Diffusion“ oder des „Sickerns“ zu charakterisieren. Sicher war dies keine „Rezeption“, wenn man „Rezeption“ als einen kontrollierten Prozess versteht. Genau hier liegt das Problem der Beziehung zwischen Medienwissenschaften und den biologischen Arbeitsgebieten, die sich mit Wahrnehmungsfragen beschäftigen. Diese Tagung zeigt, dass das Problem gesehen wird und dass es Forscherinnen und Forscher gibt, die es angehen wollen.

1 Der Text beruht auf dem gleichnamigen Beitrag zur Tagung des SFB/FK 615 und knüpft an die argumentative Form des Vortrags an.

2 Vgl. dazu Polanyi, Michael: Implizites Wissen, Frankfurt a.M. 1985 (zuerst veröffentlicht 1966 als The Tacit Dimension); Rammert, Werner: „Die Form der Technik und die Differenz der Medien. Auf dem Weg zu einer pragmatistischen Techniktheorie“, in: ders. (Hrsg.): Technik und Sozialtheorie, Frankfurt a.M./New York 1998, S. 293-326. 
Meine These lautet: Medienwissenschaftliche Einsichten könnten durch eine Zusammenarbeit mit Wahrnehmungsbiologen theoretisch und empirisch besser fundiert werden. Dies würde für Teilbereiche eine kumulative Wissenszunahme ermöglichen und die Anwendbarkeit (kommunikations- und) medienwissenschaftlicher Forschung verbessern. Der zweite Teil der These lautet: Die Probleme, für die die Medienwissenschaften Lösungen und Erklärungen suchen, sind so zentral für das Verständnis menschlichen und sozialen Verhaltens, dass sie auch die Wahrnehmungsbiologie herausfordern.

\section{Die betroffenen Disziplinen und wissenschaftlichen Arbeitsgebiete}

Um welche Arbeitsgebiete oder Disziplinen geht es, wenn hier von „Medienwissenschaften“ bzw. „Wahrnehmungsbiologie“ die Rede ist? Zwei Aufzählungen mögen zunächst genügen, für die ich natürlich keine Vollständigkeit beanspruche.

Im Bereich der Medien- und Kommunikationswissenschaften lassen sich vor allem drei Bereiche unterscheiden. Erstens gibt es die Publizistik(wissenschaft) oder auch „Kommunikations- und Medienwissenschaft" als Nachfolgerin der Zeitungswissenschaft. ${ }^{3}$ Sie deckt zusätzlich einen Teil der wissenschaftlichen Beobachtung und Analyse von Radio, Fernsehen und digitalen Medien. Stärker geisteswissenschaftlich orientiert ist dagegen die Filmwissenschaft, wobei es vielfältige Überlappungen mit der Publizistik, aber auch der sich explizit als Medienwissenschaft auffassenden Disziplin gibt, die durch die Verallgemeinerung besonders des Mediencharakters von Schrift aus den Literaturwissenschaften entstanden ist. Die Siegener Medienwissenschaft ist ein Beispiel dafür, auch wenn sie seit vielen Jahren insbesondere Fernsehen und Film einbezieht. Die dritte Gruppe wird durch die betriebswirtschaftliche, soziologische und politikwissenschaftliche Kommunikations- und Organisationsforschung gebildet. ${ }^{4}$ Auch wenn hier die Medien weniger im Vordergrund stehen, so ist doch die Bedeutung der Kommunikation und

3 Zur Vielfalt der Bezeichnungen siehe http:/www.dgpuk.de/allgemein/ selbstverstaendnis.htm\#Gegenstand.

4 Vgl. als Überblick: „Konzeptionen der Medienwissenschaften I. Kulturwissenschaft, Film- und Fernsehwissenschaft", in: Zeitschrift für Literaturwissenschaft und Linguistik (LiLi), 33, 132 (2003); „Konzeptionen der Medienwissenschaften II. Sozialwissenschaften und Informatik/Design“, in: Zeitschrift für Literaturwissenschaft und Linguistik (LiLi). 34, 133 (2004). 
ihrer Randbedingungen unbestritten, was natürlich nicht ohne Medien möglich ist.

Bei der Wahrnehmungsbiologie sind vor allem die Hirnforschung zu nennen, soweit sie sich mit Fragen der Wahrnehmung, ihrer Verarbeitung und Umsetzung in Handeln (einschließlich kommunikativem Handeln) beschäftigt. ${ }^{5}$ Hierher gehört aber auch die an die populationsgenetische Evolutionstheorie anknüpfende Soziobiologie ${ }^{6}$, die Verhaltensgenetik sowie die Evolutionäre Psychologie ${ }^{7}$, die sich seit eineinhalb Jahrzehnten in der Auseinandersetzung mit behavioristisch-kulturistischen Lerntheorien und einer ganzheitlich motivierten Ablehnung lokalisationstheoretischer Überlegungen gebildet hat. Schließlich ist in diesem Zusammenhang auch die Evolutionäre Kulturanthropologie zu nennen. Sie hält an der Eigenständigkeit der kulturellen Ebene fest, relativiert sie aber im Rahmen dualer Vererbungsmechanismen (vereinfachend formuliert: kulturelle Vererbung durch Lernen und biologische Vererbung durch Reproduktion), die ihrerseits zumindest teilweise als evolviert und einander beeinflussend verstanden werden. ${ }^{8}$

5 Vgl. dazu die Beiträge von Roth, Gerhard: Fühlen, Denken, Handeln. Wie das Gehirn unser Verhalten steuert, Frankfurt a.M. 2001; Fahle, Manfred in diesem Band, S. 61-109; Altenmüller, Eckart in diesem Band, S. 139-155; Pawelzik, Klaus: „Ist die Wahrnehmung von Schönheit biologisch erklärbar?“ (Vortrag Jahrestagung „Wahrnehmung - Kognition - Ästhetik“, SFB/FK 615, Siegen 2003).

6 Vgl. dazu die Standardeinführung von Voland, Eckart: Grundriß der Soziobiologie, Stuttgart/Jena 1993 und sein Beitrag „Das ,Handicap-Prinzip“ und die biologische Evolution der ästhetischen Urteilskraft“, in diesem Band, S. 35-60.

7 Umfassend dargestellt und diskutiert wird die Position in: Barkow, Jerome H./Cosmides, Leda/Tooby, John (Hrsg.): The Adapted Mind. Evolutionary Psychology and the Generation of Culture, New York/Oxford 1992; Buss, David M.: Evolutionary psychology. The new science of the mind, Boston 1999; Barrett, Louise/Dunbar, Robin/Lycett, John: Human Evolutionary Psychology, London 2001; Miller, Geoffrey F.: Die sexuelle Evolution. Partnerwahl und die Entstehung des Geistes, Heidelberg/Berlin 2001; Kenrick, Douglas T./Luce, Carol L.: The Functional Mind. Readings in Evolutionary Psychology, Boston 2003; Gaulin, Steven J. C./McBurney, Donald H.: Evolutionary Psychology, Upper Saddle River, New York 2004.

8 Wichtige Vertreter sind R. Boyd und P. J. Richerson (Boyd, Robert/Richerson, Peter J.: Culture and the Evolutionary Process, Chicago/London 1985) und W. H. Durham (Durham, William H.: Coevolution. Genes, Culture, and Human Diversity, Stanford 1991); vgl. zur Diskussion der Beziehung Kultur-Biologie allgemein die Beiträge in Weingart, Peter/Mitchell, Sandra D./ Richerson, Peter J./Maasen, Sabine (Hrsg.): Human by Nature. Between Biology and the Social Sciences, Mahwah/London 1997. 
Wie es einer Debatte mit vielen Teilnehmern entspricht, die durch den Kontext institutionalisierter Wissenschaft zumindest in einem gewissen Maße zu innovativen Beiträgen verpflichtet sind, differenziert sich die Diskussion in vielerlei nur schwer überschaubare Stränge. Als Ergebnis finden sich unterschiedliche Begriffe für gleiche und gleiche Begriffe für unterschiedliche Inhalte - und natürlich auch Auseinandersetzungen um diese Bezeichnungen. Damit werden nicht zuletzt auch Zuständigkeiten reklamiert und - auch das sollte man durchaus erwähnen - Ressourcen beansprucht. Für Soziologen, die an der Dynamik des Wissenschaftssystems interessiert sind, wäre es angesichts dieser Situation übrigens durchaus reizvoll, die Entwicklung der Medien thematisierenden Disziplinen aus wissenschaftssoziologischer Sicht zu analysieren, zumal sich dabei Parallelen zur Etablierung der Soziologie als akademische Disziplin mit deutlicher Distanz zur Biologie ergeben dürften. ${ }^{9}$ Hier soll jedoch ein eher immanent systematischer Weg gegangen werden. Schauen wir deshalb zunächst auf die Kommunikations- und Medienwissenschaften - wobei ich den Kommunikationsaspekt einbeziehe, auch wenn ich ihn nicht jedes Mal ausdrücklich erwähne. Anschließend wende ich mich den Arbeitsbereichen zu, die ich unter dem Überbegriff ,Wahrnehmungsbiologie“" aufgezählt habe.

\subsection{Medienwissenschaften}

Was bildet den Gegenstand der Medienwissenschaften? Stark vereinfachend geht es erstens um Inhalte, also um die Themen, Ereignisse, Geschichten usw., die man in Medienprodukten findet. Beispiele sind Liebe, Auseinandersetzungen um Ressourcen, Machtkämpfe usw. Inhalte kann man weiterhin danach unterscheiden, dies ist der zweite Aspekt, welche Formen oder Gestaltungsmerkmale für ihre Darstellung gewählt wurden. Hier geht es um Fragen wie: Welche Medien werden verwendet? Werden

9 Vgl. dazu Hejl, Peter M.: „The Importance of the Concepts of ,Organism“ and ,Evolution' in E. Durkheim's ,Division of Social Labor' and the Influence of H. Spencer", in: Maasen, Sabine/Mendelsohn, E./Weingart, P. (Hrsg.): Biologiy as Society, Society as Metaphors, Boston/London 1995, S. 155-191; Hejl, Peter M.: „Biologische Methaphern in der deutschsprachigen Soziologie der zweiten Hälfte des 19. Jahrhunderts", in: Barsch, A./Hejl, P. M. (Hrsg.): Menschenbilder. Zur Pluralisierung der Vorstellung von menschlicher Natur (1850-1914), Frankfurt a.M. 2000, S. 167-214; sowie zur Rezeption der Soziobiologie durch die „Scientific Community“ und die dabei wirksam gewordenen wissenschaftlichen und außerwissenschaftlichen Überzeugungen Segerstråle, Ullica: Defenders of the Truth. The Sociobiology Debate, New York/Oxford 2000. 
Inhalte ein- oder vielfarbig dargeboten? Welche Mittel sprachlicher, bildlicher, plastischer oder musikalischer Gestaltung werden gewählt? Drittens geht es um Produzenten medialer Angebote sowie um die Nutzer oder Rezipienten. Damit berücksichtigen die Medienwissenschaften, dass Medienprodukte von Menschen in historisch und kulturell je spezifischen Gesellschaften produziert und rezipiert werden, was angesichts der Vervielfältigung medialer Angebote und voranschreitender Globalisierung zunehmend wichtig wird.

Für jeden dieser Themenschwerpunkte kann man schließlich in deskriptiver Absicht fragen: Wie variabel ist er? Wie verändern sich Inhalte oder Gestaltungsmerkmale? Bestimmen die Produzenten ihre Rolle neu und unter welchen Einflüssen geschieht dies? Schließlich: Ändert sich die Nachfrage der Rezipienten?

Natürlich ist es trivial festzustellen, dass sich Inhalte und Darstellungsformen stets geändert haben, nicht zuletzt aufgrund medialen und gesellschaftlichen Wandels. Diese Beobachtung wird aber weitaus weniger trivial, wenn man sie mit der komplementären Beobachtung verbindet, dass durch viele Veränderungen hindurch einige häufig auftretende Inhalte und Gestaltungsaspekte durchgehalten zu werden scheinen. Es gibt also eine Dynamik der Veränderungen, die nicht stochastisch entsteht.

Das führt zur zweiten großen Fragegruppe. Ihr übergreifendes Problem lautet: Wie kann man die beobachtete Dynamik erklären? Wenn man nicht unterstellt, Medieninhalte oder Gestaltungsmerkmale folgten einer Eigendynamik, was einer Art Animismus gleichkäme, so fragt man damit nach der Ebene der Akteure: Was charakterisiert sie allgemein, d.h. als Exemplare der Art homo sapiens sapiens? Ergänzend dazu ist zu fragen: Was charakterisiert die Akteure soziokulturell, d.h. als Mitglieder einer bestimmten Gesellschaft? Schließlich ist es wichtig zu wissen, was sie individuell charakterisiert, also als im Wortsinne einzigartige Individuen? Gerade vor diesem Hintergrund wird „Wahrnehmungsbiologie“ interessant. Welche Arbeitsgebiete werden damit bezeichnet?

\subsection{Wahrnehmungsbiologie(n)}

„Ästhetik“ bezeichnete ursprünglich „die Wissenschaft vom sinnlich Wahrnehmbaren bzw. von der Sinneswahrnehmung“. In dieser Bedeutung, die vor die Reduzierung des Begriffs auf die Bezeichnung für eine „Lehre vom Schönen“ zurückgeht, verstehe ich „Wahrnehmungsbiologie“. Der Begriff bezeichnet also eine nicht zuletzt auch empirische Äs- 
thetik. Ich knüpfe damit an einen Forschungsbericht von Humberto R. Maturana aus dem Jahr 1970 an. Er trug den Titel „Biology of Cognition" und war der Vorläufer der Theorie, die später als Theorie der Autopoiese bekannt wurde. ${ }^{10}$ Wolfram K. Köck, Gerhard Roth und ich haben diesen Bericht damals übersetzt und über viele Wochen diskutiert. Wir waren von dem Text fasziniert. Auch wenn die Einsicht in die Tragweite der Überlegungen Maturanas reifen musste, erkannten wir doch unmittelbar eine im damaligen Kontext revolutionäre Sicht der Erkenntnisproblematik. Maturana argumentierte nämlich, die Wahrnehmung lebender Systeme sei weniger durch die Ereignisse in ihrer Außenwelt bestimmt als durch das Funktionieren des jeweiligen wahrnehmenden Systems selber. Dahinter stand die Einsicht, dass lebende Systeme „die Wirklichkeit" gar nicht so erkennen müssen, wie die am Paradigma der objektiven Abbildung orientierte Wissenschaft dachte. Organismen, dies war letztlich die neue Einsicht, haben vielmehr ein anderes und wichtigeres Problem zu lösen. Nicht „die“ Wirklichkeit in sich zu verdoppeln, ist ihr Problem, sondern Relevantes für ihr Überleben und ihre Reproduktion wahrzunehmen und in erfolgreiches Verhalten umzusetzen. Erst in diesem Zusammenhang erhält die Abhängigkeit jeder Wahrnehmung vom wahrnehmenden System ihre funktionale Bedeutung, ein Zusammenhang, der von jenen Konstruktivisten nicht verstanden wurde und wird, die, zunächst durchaus im Überschwang des Paradigmenwechsels, die Konstruktivität von Wahrnehmung als Beliebigkeit missverstanden und daran bis heute festhalten. ${ }^{11}$

Alle biologischen Arbeitsgebiete, die zum Verständnis dieser Prozesse etwas beitragen, gehören in meinen Augen zur „Wahrnehmungsbiologie“, auch wenn der Terminus eher selten verwendet wird.

10 Vgl. dazu Maturana, Humberto R. (Hrsg.): Erkennen: Die Organisation und Verkörperung von Wirklichkeit. Ausgewählte Arbeiten zur biologischen Epistemologie, Brauschweig/Wiesbaden 1982.

11 Vgl. dazu Hejl, Peter M.: „Konstruktivismus und Universalien - eine Verbindung contre nature?", in: ders. (Hrsg.): Universalien und Konstruktivismus, Frankfurt a.M. 2001, S. 7-67; vgl. dort auch ausführlicher zur oben angesprochenen Problematisierung mancher Auffassungen der Konstruktivität von Wahrnehmung. Hier ist nicht der Ort diesen Auffassungen für die Medienwissenschaften nachzugehen. Vielmehr mag der Hinweis darauf genügen, dass in den Medienwissenschaften die Referenz auf „Konstruktion“ weit verbreitet ist, ohne Erklärungen für die vielfältigen Übereinstimmungen der Konstruktionen auch von Konstrukteuren aus unterschiedlichsten Kulturen und Zeiten anzubieten, die nach dem letztlich behavioristischen Lernmodell der Durkheimschen Soziologie und Kulturanthropologie nicht auftreten sollten. 
Damit bestimme ich als „Wahrnehmungsbiologie“ alle im weiteren Sinne biologischen Arbeitsgebiete, in denen man Wahrnehmung und ihre Verarbeitung im Kontext der Verhaltenserzeugung erforscht. Die betreffenden Forschungsfelder besitzen einige gemeinsame Aspekte, auch wenn sie unterschiedlich betont werden: (1) Wahrnehmung wird als Leistung komplexer Systeme gesehen, (2) - mit Blick auf Menschen - geht man von Organismen aus, die in einer Umwelt evolvierten, und (3) sieht man diese Umwelt als wesentlich durch die Entstehung und Evolution der Populationen gebildet, die unsere Vorfahren konstituierten.

Vor diesem Hintergrund seien einige wahrnehmungsbiologische Arbeitsgebiete genannt und durch ihre zentrale Frage und die Bezeichnung des jeweiligen Fokus gekennzeichnet. Beginnt man mit der biologischen, neuropsychologischen oder neuroinformatischen Hirnforschung, so lautet ihre Kernfrage: Wie funktioniert das zentrale Organ für Wahrnehmung und Verhaltenserzeugung? Der Fokus ist hier das Gehirn als Organ der Wahrnehmung von internen und externen Ereignissen und ihre Umsetzung in Aktivitäten. Davon deutlich unterschieden ist die Verhaltensgenetik. Sie fragt danach, welche Verhaltensweisen eine genetische Grundlage haben und wie sie Verhalten fördert, aber auch restringiert. Der Fokus liegt auf der biologischen Vererbung von Verhalten, weshalb Verhalten hier im Zusammenhang mit der Lösung universeller (=stetig wiederkehrender) Probleme steht. Ebenfalls auf Verhaltenserklärung orientiert, wenn auch mit anderer Stoßrichtung, ist die Soziobiologie: Welche Verhaltensweisen sollte man als Ergebnis menschlicher Evolution in der evolutionär relevanten sozialen Umwelt erwarten? Der Fokus liegt hier primär auf der Evolution von Sozialität mit teilweise deutlicher Tendenz, die natürlich immer attraktive Frage von Partnerbeziehungen in den Vordergrund zu stellen. Dass der Aspekt der Evolution von Sozialität jedoch erheblich weiter führt, zeigen etwa die zahlreichen Querbeziehungen zur Spieltheorie. ${ }^{12}$ Im Unterschied zur Soziobiologie ist die Frage-

12 Gerade von diesen zunehmend empirischer werdenden Arbeitsbereichen hat in den letzten Jahren die immer wieder kritisierte starke Orientierung der Soziobiologie am Modell des ,economic man“ eine wichtige Relativierung erfahren, vgl. etwa Henrich, Joseph: „In Search of Homo Economicus: Behavioral Experiments in 15 Small-Scale Societies", in: American Economic Review, 91, 2 (2001), S. 73-78; Fehr, E./Gächter, S.: „Altruistic punishment in humans“, in: Human Nature, 415 (2003), S. 137-140; Fehr, Ernst/ Henrich, Joseph: „Is Strong Reciprocity a Maladaptation? On the Evolutionary Foundations of Human Altruism“, in: Hammerstein, P. (Hrsg.): Genetic and Cultural Evolution of Cooperation, Cambridge, Mass. 2003.

(http://www.iew.unizh.ch/home/fehr/papers/IsStrongReciprocityaMaladapta tion.pdf). 
stellung der Evolutionären Psychologie breiter angelegt. Sie fragt nach evolvierten kognitiven und emotionalen Mechanismen und danach, wie sie menschliches Verhalten beeinflussen. Nicht zuletzt durch die Traditionen der Psychologie bedingt, steht im Fokus der Evolutionären Psychologie vor allem das Individuum. Schaut man auf die Evolutionäre Kulturanthropologie so wird angesichts der gerade genannten Schwerpunkte ihre auch für die Medienforschung interessante Orientierung erkennbar. Evolutionäre Kulturanthropologen untersuchen, wie die primär analytisch unterschiedenen Ebenen der biologischen, kognitiven und sozialen Evolution zusammenwirken. Damit wird auch deutlich, dass es hier im Kern um Fragen der „dualen Vererbung“ geht, also des Zusammenwirkens von biologischer und kultureller Vererbung. Hinzu kommt zunehmend eine Anknüpfung an neuere Überlegungen zur Gruppenselektion, nachdem die Defizite strikt individuenzentrierter evolutionstheoretischer Ansätze sowohl bei der Erklärung sozialer Phänomene aus der weiteren Biologie als auch menschlichen Verhaltens unter den Bedingungen evolutionär neuer Großgesellschaften (Schlagwort „Ultrasozialität“) thematisiert wurden. ${ }^{13}$

\section{Gemeinsamkeiten}

Von einem Problem der Nicht-Beziehung zwischen Medienwissenschaften und Wahrnehmungsbiologie zu sprechen, wie es im Titel dieses Beitrages geschieht, ist nur sinnvoll, wenn man zeigen kann, dass eine Beziehung - wäre sie denn vorhanden - Vorteile brächte. Das setzt mindestens einige Gemeinsamkeiten voraus.

Tatsächlich gibt es solche Gemeinsamkeiten. Beide Wissenschaftsbereiche teilen mehr oder weniger ausgeprägt (1) ein Interesse an Menschen und menschlicher Sozialität in verschiedenen Formen. Was das jedoch genau heißt, wird erst deutlich, wenn man sich auf eine abstraktere Ebene begibt, auf der ein ganzer Bereich gemeinsamer Interessen lokalisiert ist. Medienwissenschaften und Wahrnehmungsbiologie haben

13 Vgl. zur Ultrasozialität z.B. Richerson, Peter J./Boyd, Robert: „The Evolution of Human Ultrasociality", in: Eibl-Eibesfeldt, I./Salter. F. K. (Hrsg.): Indoctrinability, Ideology, and Warfare. Evolutionary Perspectives, New York/Oxford 1998, S. 71-95 und zur Gruppenselektion Wilson, David S.: Darwin's Cathedral. Evolution, Religion, and the Nature of Society, Chicago/London 2003 sowie zum damit zusammengehenden Problem der Kooperation bzw. des Altruismus Sober, Elliot/Wilson, David S.: Unto Others. The Evolution and Psychology of Unselfish Behavior, Cambridge, Mass./ London 2003. 
nämlich (2) ein gemeinsames Interesse an Unterscheidungen, Differenzierungen und Selektionen mit Blick auf individuelle und kommunikative Konstruktionen von Wirklichkeitsvorstellungen. Dazu gehört, dass sie schließlich auch das Interesse an der Handlungsrelevanz dieser Wahrnehmungen teilen.

\subsection{Zum transdisziplinären Interesse an Menschen und menschlicher Sozialität}

Das Interesse an Menschen und menschlicher Sozialität teilen die Kommunikations- und Medienwissenschaften mit den verschiedenen wahrnehmungsbiologischen Arbeitsgebieten. Freilich darf dies nicht als inhaltliche Übereinstimmung missverstanden werden. Die Menschenbilder unterscheiden sich zum Teil deutlich, auch wenn man unterstellt, es habe insbesondere in den letzten Jahren eine Annäherung gegeben.

Trotzdem sollte man sich dieses gemeinsamen Interesses über unterschiedliche Menschenbilder hinweg bewusst sein. Das Interesse am Menschen und an menschlicher Sozialität begründet weitergehende Gemeinsamkeiten zwischen den Medienwissenschaften und den Wahrnehmungsbiologie(n), als sie mit einigen Bereichen der Physik bestehen. Denkt man z.B. an den immer wieder angesprochenen „2. Hauptsatz der Thermodynamik“ oder die berühmten „dissipativen Strukturen“, so ist keineswegs klar, worin der Gewinn solcher „Importe“ in die Sozialwissenschaften besteht, wenn man davon absieht, dass der Verweis auf sie evtl. als Beleg für Inter- oder Transdisziplinarität dienen soll.

Angesichts der verbreiteten Praxis einer zu oft vor allem terminologischen oder metaphorischen Überwindung der Differenz zwischen unterschiedlichen Disziplinen ist es notwendig, die unterschiedlichen Orientierungen von Natur- und Geisteswissenschaften anzusprechen, auch wenn die Medienwissenschaften aufgrund der Heterogenität der sie bildenden Disziplinen hier ein Bild des Pluralismus bieten. Als Soziologe habe ich nicht nur die geisteswissenschaftlich orientierte Medienwissenschaft, sondern auch die Sozialwissenschaften im Blick, die in vieler Hinsicht - und sicher in einigen ihrer Hauptströmungen - Teil der Geisteswissenschaften sind. Hier ist nicht der Ort, die Auseinandersetzungen um die unterschiedlichen Wissenschaftsverständnisse auch nur zu skizzieren. Für mein Thema genügt der Hinweis, dass wir es bei den Geisteswissenschaften in erheblichem Maße mit einer Tradition zu tun haben, die, vernachlässigt man die Entstehungsbedingungen, die der Nationalismus des ausgehenden 18. und vor allem des 19. Jahrhunderts bot, 
ideengeschichtlich dem Idealismus entstammt. Das Ergebnis ist ein Wissenschaftsdualismus, bei dem die Sozialwissenschaften teilweise recht orientierungslos zwischen den Fronten stehen. Die Situation lässt sich dadurch kennzeichnen, dass die Geisteswissenschaften trotz vieler und auch zukünftig wichtiger Leistungen für die Kulturen ihrer Länder - und darüber hinaus - sich auf einem ständigen, wenn auch diskontinuierlichen Rückzug aus einer Öffentlichkeit befinden, in der geisteswissenschaftliche Terrains, soweit es um Sinngebung geht, scheinbar zunehmend von religiöser Seite eingeschränkt werden, ${ }^{14}$ während ihre Rationalisierungsdiskurse von naturwissenschaftlicher Seite auf- und übernommen wurden. Ausdruck für diesen Schrumpfungsprozess ist die permanente Diskussion um das Selbstverständnis und die Funktion der Geisteswissenschaften in der heutigen Zeit als eine Selbstvergewisserung innerhalb einiger ${ }^{15}$ geisteswissenschaftlicher Disziplinen. Natürlich wird diese Diskussion auch um das Selbstverständnis und die Rolle der Naturwissenschaften geführt, charakteristischerweise aber als eine Diskussion, die - Beispiel Ethikkommissionen in den medizinischen bzw. biologischen Fakultäten oder die „Enquete-Kommission „Ethik und Recht der modernen Medizin" - als politisch, ethisch und wirtschaftlich folgenreich angesehen wird und die deshalb auch außerhalb der Naturwissenschaften im engeren Sinne Interesse und Engagement auslöst. ${ }^{16}$ Man geht in der Breite der Gesellschaft davon aus, dass diese Disziplinen Ergebnisse erzielt haben, und weiter produzieren werden, die ob ihrer Folgen nicht nur wissenschaftsintern sondern auch öffentlich diskutiert werden müssen. Angesichts dieser Differenz in der öffentlichen Aufmerksamkeit und Bewertung können die Geisteswissenschaften sich auf Lehrerausbildung und Beiträge zur Binnendiskussion zurückziehen oder, wie u.a. mit der

14 Eine Reihe gesellschaftlicher Entwicklungen zu Beginn dieses Jahrtausends lassen die Frage entstehen, ob nicht in einigen Jahren der Blick auf die Jahre der „Postmoderne“ und der damit einhergehenden Beliebigkeit diese als den Übergang zu sich ausbreitender Religiosität und zu einem Sicherheiten versprechenden Konservatismus gesehen werden. Darauf, dass die oft angesprochene Säkularisierung der Moderne auf einer Verwechslung der abnehmenden Bedeutung religiöser Institutionen mit einem Rückgang von religiösen Interessen beruht, hat bereits 1993 R. St. Warner (Warner, R. Stephen: „Work in Progress toward a New Paradigm for the Sociological Study of Religion in the United States", in: American Journal of Sociology, 98 (1993), S. 1044-1093) verwiesen.

15 So scheinen Geschichtswissenschaft oder Linguistik weniger betroffen zu sein.

16 Zur Einschätzung der Relevanz vgl. den Einsetzungsbeschluss des Deutschen Bundestages vom 18.2.2003, Drucksache 15/464. 
Medienwissenschaft begonnen, sich anderen Themen öffnen und damit auch die mit ihnen verbundene Relevanz beanspruchen. Damit stellt sich jedoch unausweichlich das Problem der unterschiedlichen Wissenschaftsauffassungen, also das Problem des Wissenschaftsdualismus.

Ohne eine Lösung des Dualismusproblems wird es, dies ist meine feste Überzeugung, kaum eine produktive Annäherung zwischen Medienwissenschaften und Wahrnehmungsbiologie geben. Sieht man von individuellen Präferenzen ab, etwa im Zusammenhang mit empirischen Verfahren, die zu reproduzierbaren Ergebnissen führen, so lassen sich für die Überwindung des Dualismus Argumente anführen, für die man zumindest beanspruchen kann, dass sie, obwohl seit einigen Jahren vorliegend, noch kein Allgemeingut sind. Dies möchte ich an zwei zentralen Positionen deutlich machen: dem heute immer stärker in den Vordergrund tretenden Menschenbild und der damit zusammenhängenden Auffassung von Kultur.

Die evolutionstheoretisch informierte anthropologische und teilweise auch primatologische Diskussion der letzten Jahrzehnte hat zu konzeptionell bedeutsamen Veränderungen älterer Auffassungen geführt. Lange nahm man unter dem Einfluss des Marxismus an, die für die menschliche Evolution relevante Umwelt sei die physische Umwelt. Der Mensch wird in diesem Zusammenhang als ein Wesen gedacht, das seiner physischen Umwelt die zum Überleben benötigten Ressourcen abringt. Die Auseinandersetzung mit ihr und die Anpassung an sie werden dabei als zentrale Faktoren der menschlichen Evolution gesehen. Wichtig ist in diesem Zusammenhang, sich bewusst zu machen, dass diese Orientierung auf die Beziehung Mensch/natürliche Umwelt nicht länger die vorherrschende Meinung ist. Im Zusammenhang mit der sogenannten ,social intelligence hypothesis ${ }^{\text {“17 }}$ geht man heute davon aus, die wichtigste Umwelt der menschlichen Evolution sei nicht physischer, sondern sozialer Natur gewesen. Aus dieser Sicht sind wir biologisch und damit auch kognitiv und emotional das Resultat der Evolution in den sozialen Gruppen, die unsere Vorfahren gebildet haben: Menschliche

17 Der Klassiker dazu ist Byrne, Richard W./Whiten, Andrew (Hrsg.): Machiavellian Intelligence. Social Expertise and the Evolution of Intellect in Monkeys, Apes, and Humans, Oxford 1988. Der Band vereinigt zahlreiche Arbeiten zum Thema. Vgl. auch Byrne, Richard W.: The thinking ape. Evolutionary Origins of Intelligence, Oxford 1995; Dunbar, Robin I. M.: Klatsch und Tratsch. Wie der Mensch zur Sprache fand, München 1998. Vgl. kritisch dazu Kummer, Hans/Dasser, Verena/Hoyningen-Huene, Paul: „Exploring Primate Social Cognition: Some Critical Remarks“, in: Behavior, 112 (1990), S. 1-2. 
Biologie und menschlicher „Geist“ haben einen koevolutiven Ursprung. Dies ist letztlich gemeint, wenn gesagt wird, die tradierten Dualismen seien obsolet geworden.

Setzt man diese Überlegungen fort, so lässt sich Kultur mit der evolutionären Kulturanthropologie bzw. mit der Evolutionären Psychologie als eine evolutionäre Errungenschaft verstehen. Durch sie wurde es möglich, folgt man z.B. Robin I. M. Dunbar ${ }^{18}$, die geringen Gruppengrößen zu überschreiten, in denen der größte Teil menschlicher Evolution stattfand. ${ }^{19}$ Mit Kultur entstand ein zweites Vererbungssystem - wenn man Kultur inhaltlich als die Menge der Prozesse versteht, durch die menschliches Wissen kommuniziert und damit weitergegeben wird. Diese kulturelle Wissensweitergabe operiert erheblich schneller als der biologische Transfer von Eigenschaften. Gleichzeitig beruht kulturelle Vererbung aber auf kognitiven Mechanismen, für die natürlich eine evolvierte biologische Grundlage anzunehmen ist, auch wenn die Wahrnehmungsbiologie die Komplexität der hier relevanten Verarbeitungen allenfalls teilweise entschlüsselt hat.

Damit komme ich zur zweiten Ebene der Gemeinsamkeiten zwischen Medienwissenschaften und Wahrnehmungsbiologie, dem Interesse an erfolgreichen Unterscheidungen und Selektionen. Die Gemeinsamkeiten, um die es hier geht, bauen auf den Veränderungen der Dualismen auf, die oben skizziert wurden.

\subsection{Interesse an erfolgreichen Unterscheidungen und Selektionen}

Im Anschluss an das, was ich schon zum Gegenstandsbereich der Medienwissenschaften gesagt habe, werde ich zunächst auf die unterschiedlichen Selektionsprobleme eingehen. Parallel dazu werde ich eine Reihe von Fragen formulieren, die an die Wahrnehmungsbiologie zu stellen sind, wobei es sich natürlich um eine „offene Liste“ handelt. Ich hoffe, damit inhaltlich zur Überwindung der Nicht-Beziehung anzuregen.

Die erste Frage, die gerade mit Blick etwa auf das Handeln von Journalisten oder Medienunternehmen immer wieder gestellt wird, lautet natürlich: Wer selektiert? Die normale Antwort besteht meist im mehr oder weniger differenzierten Verweis einerseits auf „Produzenten“ und andererseits auf „Rezipienten bzw. Nutzer“. Natürlich spielen hier auch institutionelle, ökonomische und deontologische Fragen eine erhebliche

18 Dunbar (wie Anm. 17).

19 Vgl. dazu Anm. 12. 
Rolle. Darauf kann hier jedoch nicht eingegangen werden. Stattdessen sei gefragt: Was wird selektiert? Hier kann man, je nach Fragestellung, unterschiedlich klassifizieren. Verbreitet ist die Unterteilung in „Information“ und „Unterhaltung“. Tatsächlich ist diese Unterscheidung jedoch eher problematisch. Was den einen unterhält, kann den anderen informieren und umgekehrt. Tatsächlich spricht einiges dafür, dass die Unterscheidung einerseits einem kulturellen Kontext entstammt, in dem sowohl christlich-fundamentalistische als auch marxistische Traditionen zusammenwirken, nach denen „Unterhaltung“ als „weltlich“, „eskapistisch“ oder auf „Verführung“ durch eine „Kulturindustrie“ verstanden wird. Mit Blick auf Deutschland ist überdies zu berücksichtigen, dass die Dominanz der Unterscheidung „Information“ - „Unterhaltung“ auch mit Legitimationsnotwendigkeiten einhergeht, die sich aus den Bestimmungen des Rundfunkstaatsvertrags (2002) ergeben und die sich z.B. in einem unterschiedlichen Informationsverständnis von öffentlich-rechtlichen und privaten Fernsehanbietern niederschlagen (U. M. Krüger 2000).

Der Unterscheidung Information/Unterhaltung ist aber zunächst eine andere vorgeschaltet: die zwischen ,interessant“/,nicht interessant“ (Hej1 2003). Nur was interessant ist, wird anschließend als „Information“ bzw. „Unterhaltung“ verarbeitet. Wie lassen sich aber Information bzw. Unterhaltung bestimmen? Geht man von einer konstruktivistischen Position aus ${ }^{20}{ }^{20}$ so lässt sich die Unterscheidung anhand der folgenden Bestimmung treffen:

Wenn der Unterschied, den ein Medienangebot für einen Nutzer ausmacht, in einer Veränderung seines eigenen Zustandes besteht, ohne daß er einen weiteren Handlungsbedarf sieht, so handelt es sich um Unterhaltung. Unterhaltung ist von der Verwendung des kommunikativen Angebotes her also Selbstzweck im Sinne konsumatorischen Handelns. In jedem anderen Fall geht es um Informa-

20 Die Literatur zum Konstruktivismus wird hier als bekannt vorausgesetzt. Interessant ist in dem Zusammenhang aber, dass es kaum empirische Arbeiten zum hier angesprochenen Thema gibt. Eine nicht aus dem Kernbereich konstruktivistischer Medienforschung stammende Untersuchung (Kepplinger, Hans M./Tullius, Christiane: ,Fernsehunterhaltung als Brücke zur Realität. Wie die Zuschauer mit der Lindenstraße und dem Alten umgehen“, in: Rundfunk und Fernsehen, 43, 2 (1995), S. 139-157) enthält jedoch Material, das die oben präsentierte These stützt und bezüglich der Befunde zur Geschlechtsspezifik von Konstruktionsprozessen darüber hinaus starke anthropologische Implikationen hat, die die Autoren allerdings vermeiden anzusprechen. 
tion. Sie wird vom Nutzer immer als Verweis auf anderes gesehen, oft als (tatsächlich oder potentiell) handlungsrelevant. ${ }^{21}$

Wenn man nun bei „konsumatorischem Handeln“ an Prozesse denkt, bei denen Neurotransmitter mit unterschiedlichen Wirkungen produziert werden, so sollte es Möglichkeiten geben, auf biologischer Ebene verschiedene Arten medialer Unterhaltung zu präzisieren. Darauf zielen die Fragen:

- Wie funktionieren „Interesse“, „Erwartung“, „Bedeutung“, „Relevanz“, „Emotion“?

- Was ist „Unterhaltung“ aus biologischer Sicht?

- Wie lassen sich unterschiedliche Arten von „Unterhaltung“ bestimmen?

Schauen wir uns jetzt genauer an, was selektiert wird, wobei man zwischen Inhalten und/oder Gestaltungsalternativen unterscheiden kann, sowie, besonders bei Film und Fernsehen, den Ausführenden (auf die ich hier nicht eingehe). Zunächst, welche Inhalte werden selektiert?

Prinzipiell gibt es zwei Selektionsmöglichkeiten. Man kann zwischen vorhandenen Möglichkeiten auswählen oder sich für Innovation entscheiden. In der Regel dürfte es sich um eine Kombination aus der Variation von Bekanntem und kleineren Neuerungen handeln, wobei die bekannten Themen deutlich dominieren. Schaut man auf sie, so finden sich im Bereich der Unterhaltung so zeitlose Themen wie „Mann trifft Frau“, „Kampf um Gerechtigkeit“, „Lob der Freundschaft“ etc. Der Informationsbereich wird dagegen von Fragen beherrscht wie: „Wer gegen wen?“ „Welche Allianzen?“, „Wer ist erfolgreich?“, sowie allgemein: „Was kommt auf mich zu?“ Bei den Innovationen oder Erfindungen geht es primär um Unterhaltung - auch wenn ,erfundene Informationen“ immer wieder vorkommen. Überdies können Erfindungen zum Zweck der Unterhaltung Anlass für Aktivitäten geben, die dann zu Informationen werden.

Angesichts dieser Inhalte ergibt sich eine sicher ergänzbare Reihe von Fragen an die Wahrnehmungsbiologie im hier verwendeten weiteren Sinne:

- Für welche Inhalte kann man mit Interessen rechnen?

- Wie kann man Arten von Interessen unterscheiden?

21 Vgl. dazu ausführlicher Hejl, Peter M.: „Unterhaltung als Information, Information als Unterhaltung“, in: Hofmann, W. (Hrsg.): Die Sichtbarkeit der Macht. Theoretische und empirische Untersuchungen zur visuellen Politik, Baden-Baden 1999, S. 108-123. 
- Welche Interessen treten bei welchen Inhalten auf?

- Wie ändern sich Interessenstrukturen im Lebenslauf?

- Welche Zusammenhänge zwischen dem Interesse an Inhalten und dem Geschlecht der Interessenten gibt es?

- Welche wahrnehmbaren Merkmale wirken als Auslöser, so dass Rezipienten auf spezifische Inhalte schließen?

- Welche Inhalte werden als zusammengehörig wahrgenommen?

- Welche Inhalte erzeugen die Wahrnehmung von Wohlbefinden, welche die von Unwohlsein?

Schaut man nun auf die Selektion von Gestaltungsmöglichkeiten, so ergeben sich wieder verschiedene Entscheidungsoptionen. Zunächst gibt es natürlich die Auswahl zwischen verschiedenen Medien und Medienkombinationen. Im Bereich der nonverbalen Kommunikation haben wir so z.B. Mimik und Gestik, wie man sie kombiniert und intensiviert in der Pantomime findet. Wir haben die auditiven Medien Musik und im Bereich der verbalen Kommunikation natürlich die Sprache mit Schrift bzw. dem Druck als einer visuellen Repräsentation des akustischen Mediums Sprache. In den visuellen Bereich gehören aber natürlich auch die Bilder, die schließlich ,laufen lernten“ und in der Kombination von auditiven und visuellen Darstellungen mit Film und Fernsehen eine dominante Position erlangten. Zur Gestaltung gehören aber auch die Möglichkeiten, Themen oder Inhalte allgemeinen oder gar universellen Interesses durch ganz unterschiedliche Inhalte zu konkretisieren. Man schaue nur auf das Thema Partnerwahl, sicher eines der Themen, das kaum je auf Desinteresse stößt, auch wenn jeder weiß, worum es geht. Bekannte Beispiele für die Variationsmöglichkeiten sind etwa Aschenputtel, Romeo und Julia, Froschkönig oder die vielen Varianten von „la belle et la bête“. Schließlich kann auf Sprache oder Musik bzw. Gesang und damit mögliche wechselseitige Kontextuierungen zurückgegriffen werden. ${ }^{22}$ Schließlich werden nicht zuletzt im Zusammenhang mit technischen Innovationen Gestaltungsmöglichkeiten erfunden oder verändert. Beispiele sind Computeranimationen oder neue Formen etwa in der Malerei, wobei häufig traditionelle Motive mit neuer Pinselführung dargestellt werden, Abstraktionen auftauchen, etwa geometrische Formen wie im Kubismus, oder bestimmt Formen oder Strukturen in den Vordergrund rücken. Schließlich findet man die Kreation neuer sprachlicher, bildlicher oder musikalischer Ausdrucksweisen. Auch hier stellt sich wieder eine Reihe

22 Als poetisch-melancholisches Beispiel kann man hier Alain Resnais' Film ON CONNAIT LA CHANSON (1997) nennen. 
von Fragen, wobei ich besonders auf Emotionen und Verständlichkeit eingehe:

- Wie beeinflusst hohe Emotionalität oder Stress die Wahrnehmung von Inhalten?

- Wie funktioniert der „Transfer von Emotionen“, d.h. welche Merkmale von Medienangeboten lösen wie Emotionen bei den Rezipienten aus?

- Lassen sich Emotionen nach ihrer Relevanz (wofür?) klassifizieren?

- Insbesondere: Wie ist das Phänomen „Angstlust“ zu erklären?

- Wie beeinflussen sich verschiedene Wahrnehmungsmodalitäten (Bild/Sprache/Musik)?

- Wie wirken unterschiedliche Medien auf kognitive bzw. emotionale Prozesse?

Betrachten wir nun Selektionen, deren Gründe primär nichts mit Inhalten oder Gestaltungsmöglichkeiten zu tun haben, deren Gratifikationen also anderen Bereichen entstammen. Hier lassen sich vor allem zwei Klassen unterscheiden, auch wenn es im Einzelfall schwierig sein dürfte, genaue Abgrenzungen zu finden. Selektionsgründe können überwiegend „in“ den Akteuren liegen, also „persönlicher Natur“ sein, oder überwiegend in ihrer sozialen Umwelt zu suchen sein.

Bei den Selektionsgründen eher persönlicher Natur lässt sich zwischen der Auswahl aus einem Bestand und Innovationen differenzieren: Angesichts der bereits angesprochenen vielfältigen Einflüsse, die auf die Steuerung ,individuellen“ oder „persönlichen“233 Handelns einwirken, wird deutlich, wie schwierig jede Bestimmung von „Person“ als Zurechnungseinheit ist. Sicher sind wir alle „Individuen“ im Sinne einer nicht hintergehbaren Einzigartigkeit. Wieweit diese jedoch für ,mehr“ als die Modulation unseres Handelns anzusetzen ist, ist unklar (s.u.). Geht man also von einem „Bestand“ an Wahlmöglichkeiten auf der Akteursebene aus, so kann man als Motive für unterschiedliche Selektionen ein eigenes Interesse an einem Thema ansetzen, aber auch Routine und den damit verbundenen Gewinn an Zeit und Ressourcen bei gleichzeitiger Stressreduzierung. Schließlich sollten auch opportunistische Gründe als normal

23 Zur Diskussion um die Differenz von „Individuum“ und „Person“ vgl. die Beiträge in Carrithers, Michael/Collins, Steven/Lukes, Steven: The Category of the Person. Anthropology, Philosophy, History, Cambridge 1985; Luhmann, Niklas: „Die Form ,Person““, in: Soziale Welt, 42, 2 (1991), S. 166-175. 
und wichtig angesehen werden, etwa solche, die mit der Sicherung des Lebensunterhalts einhergehen.

Auf dieser Ebene findet sich aber auch der Wunsch nach Neuem, etwa aus Langeweile durch Wiederholung. Selbst wenn dieser Fall sehr viel seltener sein dürfte, als oft angenommen wird - meist handelt es sich bei auch als ,innovativ“ charakterisierten thematischen Innovationen um Variationen aus dem Fundus der fundamental gleichen Themen -, kann und soll er zumindest als Wunsch nicht ausgeschlossen werden. Senkt man dagegen den Anspruch an die Radikalität von „Neuem“, so öffnet sich ein weites Feld von Innovationen. Man findet hier z.B. die Faszination durch Ideen oder Erfahrungen, die aus einem anderen Bereich in den transferiert werden, in dem man sie als innovativ einstuft. Beispiele sind die Entstehung der Radioastronomie aus der Verbindung der Radartechnologie mit Interessen von Astronomen nach dem Ende des Zweiten Weltkriegs ${ }^{24}$ oder der Import von Theorien oder Methoden aus den Sozialwissenschaften und der Psychologie in die Medienwissenschaften, ein Transfer von „härteren“ in „,weichere“ Disziplinen, für den es einige Beispiele gibt.

Neben diesen eher dem persönlichen Handeln zuzuordnenden Selektionen finden sich aber auch solche, die sehr viel deutlicher einen sozialen Ursprung haben bzw. damit zusammenhängen, dass die Regelmäßigkeiten der sozialen Umwelt individuelle Abweichungen profitabel machen. ${ }^{25}$ So sind viele Wahlen, die als individuelle Entscheidungen

24 Vgl. dazu die klassische Untersuchung von M. J. Mulkay und D. O. Edge (Mulkay, Michael J./Edge, David O.: „Cognitive, Technical and Social Factors in the Growth of Radio Astronomy“, in: Social Science Information, 12, 6 (1973), S. 25-61.

25 Vgl. zum Problem der Nicht-Kooperation aus spieltheoretischer Sicht neben den Angaben in den Fußnoten 11f. den Klassiker Axelrod, Robert: Die Evolution der Kooperation, München 1988 sowie Fehr, Ernst/Gächter, Simon: „Altruistic punishment in humans“, in: Human Nature, 415 (2003), S. 137140. Aus evolutionspsychologischer Sicht vgl. Cosmides, Leda/Tooby, John: „Cognitive Adaptations for Social Exchange“, in: Barkow, J. H./Cosmides, L./Tooby, J. (Hrsg.): The Adapted Mind: Evolutionary Psychology and the Generation of Culture, New York 1992, S. 163-228; Cosmides, Leda/Tooby, John: „From Evolution to Adaptations to Behavior: Toward an Integrated Evolutionary Psychology“, in: Report der „, Research Group on Biological Foundations of Human Culture “ Bielefeld, 4 (1992); Gigerenzer, Gerd/Hug, Klaus: „Domain-Specific Reasoning: Social Contracts, Cheating, and Perpective Change“, in: Report der ,, Research Group on Biological Foundations of Human Culture at the Center for Interdisciplinary Research" Bielefeld, 5 (1992); Price, Michael E./Cosmides, Leda/Tooby, 
präsentiert werden, natürlich auch der Anpassung an eine soziale Umwelt geschuldet, was - nicht zuletzt im Medienbereich - auch mit der Zugehörigkeit zu sozialen Netzwerken einhergeht. Dementsprechend findet man einerseits die Anpassung an Teile der sozialen Umwelt (Konformität mit der dominierenden Kultur bzw. mit einer Sub- oder Teilkultur) und andererseits Unterscheidung oder Absetzung von Teilen der sozialen Umwelt. Wie spätestens seit den kultursoziologischen Arbeiten Bourdieus zur „Distinction“ auch über die Fachgrenzen bekannt, signalisieren solche Abweichungen von den Erwartungen der Mehrheit die Zugehörigkeit der Abweichenden zu einer Elite, ${ }^{26}$ ein Mechanismus, der auf dem Spannungsverhältnis von Anpassung und Individualismus beruht, wie ihn bereits G. Simmel in seiner Theorie der Mode (1919) anspricht.

\section{Der aktive Mediennutzer und das Problem der Willensfreiheit}

Die Fragestellungen der an den Massenmedien orientierten Wirkungsforschung haben sich in den letzten Jahrzehnten deutlich verändert. Lange Zeit ging man von der Annahme eines passiven Rezipienten aus. Man sah ihn als den Medien ausgeliefert und, dies die ideologiekritische Implikation, durch die Medien manipuliert. Dieses Bild wurde in den letzten Jahren in breiten Teilen der Forschung durch die Annahme eines Akteurs ersetzt, der aktiv auswählt und vor dem Hintergrund seiner Interessen Angebote bewertet. Aus dem passiven Rezipienten wurde damit ein aktiver Mediennutzer. Diesem aktiven Nutzer wurde mehr oder weniger ausdrücklich unterstellt, „frei“ zu entscheiden - und sei es durch den Verzicht, aus dem Medienangebot auszuwählen: der Druck auf den AusKnopf!

Das Menschenbild des „aktiven (=freien) Mediennutzers“ passt zweifellos besser zu unserem Selbstverständnis als „freie Bürger“ in einer "freien Gesellschaft“ als frühere Positionen, die stärker davon ausgingen, dass die Mediennutzer durch die Medien nachhaltig beeinflusst würden. Konfrontiert man die Grundannahmen der Vorstellung vom aktiven Nutzer jedoch mit Arbeiten und Überlegungen zur Willensfreiheit,

John: „Punitive sentiment as an anti-free rider psychological device“, in: Evolution and Human Behavior, 23, 3 (2002), S. 203-231.

26 Für dieses Verhalten wurde mit der „Theorie der teuren Signale“ bzw. des „Handicap-Prinzips“ von A. Zahavi auch eine inzwischen vielfach bestätigte evolutionsbiologische Theorie vorgelegt, vgl. dazu Uhl, Matthias/Voland, Eckart: Angeber haben mehr vom Leben, Heidelberg/Berlin 2002. 
wie sie in den letzten Jahren aus der Hirnforschung kommen, so erscheint das Bild der freien Entscheider erneut und grundsätzlich revisionsbedürftig.

Wie G. Roth ausführt ${ }^{27}$, liegen gut bestätigte empirische Befunde vor, die als Widerlegung des traditionellen Konzepts der Willensfreiheit gelten müssen. Logisch betrachtet, impliziert Willensfreiheit, dass der „freie Willensakt“ der Beginn der damit beschlossenen Handlung sein muss. Im Gegensatz dazu zeigen von Benjamin Libet und Mitarbeitern 1983 vorgelegte und seitdem mehrfach wiederholte und verbesserte Untersuchungen, dass dies nicht der Fall ist. Der Beschluss, etwas zu tun, geht der Einleitung der betreffenden Tätigkeit nicht voraus. Vielmehr folgt er ihr um einen Sekundenbruchteil. Das bedeutet: Als Systeme handeln wir bereits, bevor in dem Teilsystem, das für die Bewusstmachung von Denkprozessen zuständig ist, die Wahrnehmung entsteht: "Ich habe entschieden“. Obwohl kontraintuitiv, ist dieser Befund systematisch überzeugend. Anlagen und Erfahrungen können nur deshalb ihre überwiegend positiven Wirkungen entfalten, weil es keine Willensfreiheit im Sinne freier Setzungen gibt. Sie würden nämlich Erfahrung und Lerneffekte außer Kraft setzen und damit eine wichtige Steuerungsmöglichkeit autonomisierter Systeme behindern. Die skizzierten Befunde zur Willensfreiheit und Autonomie haben natürlich eine Fülle von Implikationen. Ich möchte abschließend nur auf zwei Folgen eingehen, die für unser Thema besonders wichtig sind.

Sprache dient sicherlich der Rechtfertigung „in uns“ getroffener Entscheidung in sozialen Kontexten. Aus sozialwissenschaftlicher aber auch aus anthropologischer Sicht sind Medien Mittel der Kommunikation. Dies bedeutet, so die sicher dominierende Vorstellung, dass ihre Funktion primär sozialer Natur ist. Als Träger kommunikativer Angebote dienen sie dazu, Verhalten zu koordinieren und eigene Zustände, Wünsche, Absichten und Beurteilungen der sozialen Umwelt zu signalisieren. Wenn unsere Selbstwahrnehmung als „freie“ Entscheider an die Wahrnehmung gebunden ist, dass wir entschieden haben - was auf der Ebene bewusster Feststellungen und des bewussten Abwägens von pro und contra kaum ohne Sprache geschieht -, so wird eine andere Funktion vor allem von Sprache deutlich, auf die auch G. Roth verweist. Sie dient

27 Roth, Gerhard: Fühlen, Denken, Handeln. Wie das Gehirn unser Verhalten steuert, Frankfurt a.M. 2001, S. 435ff.; Singer, Wolf: „Über Bewußtsein und unsere Grenzen. Ein neurobiologischer Erklärungsversuch“, in: Becker, A. (Hrsg.): Gene, Meme und Gehirne. Geist und Gesellschaft als Natur, Frankfurt a.M. 2003, S. 279-305. 
dann in erheblichem Maße der Rechtfertigung von Alternativen, die auf unbewussten (angeborenen und gelernten) Präferenzen beruhen und über die auch an anderer Stelle entschieden wird, vermutlich im limbischen System. Diese Rechtfertigungsfunktion hat Sprache als wichtigstes Medium sicherlich in den vielen sozialen Kontexten, in denen wir unser Verhalten durch Sprache koordinieren, also Alternativen identifizieren und wieder reduzieren. So, wie das Bild des aktiven Mediennutzers zu unserem kulturell bedingten Selbstverständnis passt, so finden wir es plausibel und auch wünschbar, dass unsere sozialen Aushandlungen „vernünftig“ verlaufen, also sachliche Argumente und Gegenargumente heranziehen, sie abwägen und dann rational nachvollziehbar zu Übereinstimmungen gelangen. Natürlich wissen und beklagen wir oft genug, dass viele Aushandlungsprozesse ,irrational“, „emotional“ und an persönlichen Interessen orientiert verlaufen. Wenn Sprache auch der sozialen Rechtfertigung der Folgen nicht bewusst wirkender Präferenzen dient, dann ist eben dieser so oft unbefriedigende Verlauf zu erwarten. ${ }^{28}$

Es gilt jedoch noch eine weitere Folge zu bedenken. Die Rechtfertigungsfunktion von Sprache dürfte entgegen dem besonders sozialwissenschaftlichen Kommunikationsverständnis keineswegs auf soziale Beziehungen beschränkt sein. Im Gegenteil, man wird komplementär als zusätzliche Funktion die Selbstverständigung über externe und interne Sachverhalte annehmen müssen. Ein vielleicht paradigmatischer Fall sind die sogenannten ,Aha'-Erlebnisse. Man beschäftigt sich mehr oder weniger bewusst mit einem Problem. Plötzlich „hat man die Lösung“. Will man jedoch wissen, warum man die Lösung „hat“, braucht man Sprache als Mittel der Selbstverständigung.

Bleibt man nun beim Mittelcharakter von Medien und berücksichtigt, dass unterschiedliche Medien unterschiedliche Bereiche unseres Wahrnehmungssystems aktivieren, so muss man einen engen Zusammenhang erwarten zwischen Wirklichkeitskonstruktion, Handlungsvorbereitung bzw. -rechtfertigung und der Medienwahl, auch auf der Ebene der Kommunikation mit uns selbst bzw. der „Kommunikation in uns“. Die Folgen dieser Überlegung können hier nicht ausgeführt werden. Es muss genügen, darauf zu verweisen, dass aus Sicht der Medienwissenschaften damit der Zusammenhang zwischen unterschiedlichen Sinnesmodalitäten, Medien und Medienkombinationen sowie Wahrnehmungs-

28 Vor dem Hintergrund dieser Überlegungen zu Sprache als Medium sozialer Rechtfertigung wäre auch eine Neulektüre von L. Boltanski und L. Thevenot (vgl. Boltanski, Luc/Thevenot, Laurent: De la Justification. Les économies de la grandeur, Paris 1991) angezeigt. 
und Denkprozessen angesprochen ist. Damit ist aber auch die Wahrnehmungsbiologie involviert, und zwar auf der Ebene der Evolution, aber auch der Lokalisation unterschiedlicher Wahrnehmungssysteme und ihrer Interaktionen miteinander sowie ihres funktionalen Beitrags zur Generierung von Verhalten und Handeln.

Kehren wir abschließend zum Aspekt der Selektivität zurück. Die Arbeiten und Überlegungen zur Willensfreiheit stellen nicht in Frage, dass Medienproduktion und -nutzung vielfach konditionierte Selektionen einschließen. Problematisch wird jedoch die Frage „Wer selektiert?“ Wenn der traditionellerweise unterstellte „freie Akteur“ oder „,freie Entscheider“ in unserer Wahrnehmung als „Ich“ oder als „meine freie Entscheidung" dominiert, ihm faktisch aber nur eine zwar wichtige aber nicht entscheidende Rolle bei der Handlungserzeugung zukommt, dann muss auch die „Freiheit“ des Mediennutzers diskutiert und wahrscheinlich neu bestimmt werden. Was vor diesem Hintergrund für die Mediennutzer gilt, gilt eo ipso auch für die Medienmacher. Damit ergeben sich mindestens drei Fragen, die ich statt einer Zusammenfassung an Medienwissenschaften und Wahrnehmungsbiologie richten möchte:

- Wer oder was wählt Inhalte oder Gestaltungen aus?

- Wer oder was ist kreativ?

- Worin besteht die zurechenbare Leistung „Kreativität“? 



\section{A UTORENVERZEICHNIS}

\section{Prof. Dr. Eckhart Altenmüller}

Direktor des Instituts für Musikphysiologie und Musiker-Medizin der Hochschule für Musik und Theater Hannover. Nach dem Medizinstudium in Tübingen, Paris und Freiburg/Brsg. und dem zeitgleichen Musikstudium an der Musikhochschule Freiburg (Hauptfach Querflöte) promovierte er 1983 über die Gangentwicklung bei Kleinkindern. Während der Assistenzzeit in der Abteilung für klinische Neurophysiologie in Freiburg entstanden die ersten Arbeiten zur Hirnaktivierung beim Musikhören. Von 1985 bis 1994 absolvierte Prof. Altenmüller an der Universität Tübingen die Facharztzeit für Neurologie und habilitierte sich 1992 im Fach Neurologie. Seit der Berufung nach Hannover 1994 sind zahlreiche Arbeiten zum auditiven und sensomotorischen Lernen, zur Störung der Musikverarbeitung nach Schlaganfällen und zur emotionalen Verarbeitung von Musik entstanden.

\section{Prof. Dr. Karl Clausberg}

Studierte Ingenieurwissenschaften in Berlin und Hannover, dann Kunstgeschichte in Hamburg, London und Wien. War Aby-WarburgStipendiat in London, promovierte 1974 zum Dr. phil. der Universität Wien. Ab 1977 Lehrtätigkeit an den Universitäten Hamburg, Kassel, Osnabrück, Regensburg, Trier, Tübingen und an der Northwestern University Evanston/USA. Professor für Kunst- und Bildwissenschaften an der Universität Lüneburg.

\section{Prof. Dr. Manfred Fahle}

Studierte Biologie und Medizin in Göttingen, Gießen, Mainz und Tübingen. Verbrachte vier Jahre am Max-Planck-Institut für Biologische Kybernetik. Ab 1981 Leiter des Elektrophysiologischen Labors an der Universitäts-Augenklinik in Tübingen. Von 1989 bis 1990 am „Center for Biological Information Processing“ am Massachusetts Institute of Technology (MIT). Seither Grundlagenforschung zur Funktion des menschlichen Sehsystems. Dabei Arbeit mit psychophysiologischen Wahrnehmungsexperimenten, funktioneller Bildgebung und Summenpotenzial-Elektrophysiologie. Seit 1999 Leiter des Instituts für Hirnforschung IV und der Einheit für Human-Neurobiologie an der Universität Bremen. Hauptuntersuchungsgegenstand ist die Arbeitsweise des menschlichen Gehirns: wie es komplexe visuelle Muster analysieren kann bzw. welche Teile des Gehirns für welche Aspekte zuständig sind. 
Prof. Dr. Siegfried Frey

Professor für Kommunikations- und Medienpsychologie und Leiter des Laboratoriums für Interaktionsforschung am Institut für Kognition und Kommunikation der Universität Duisburg-Essen. Zahlreiche Veröffentlichungen zu Theorie und Methodik der humanwissenschaftlichen Kommunikationsforschung. Ausgezeichnet mit dem „Forschungspreis Technische Kommunikation" der Alcatel SEL-Stiftung, Stuttgart. Lehr- und Forschungstätigkeit am Max-Planck-Institut für Psychiatrie, München, an der University of California, San Francisco, an der Universität Bern und an der Ecole des Hautes Etudes en Sciences Sociales, Paris. Seit 1999 Vorsitzender des Vorstands der Europäischen Akademie für multimediales Lernen in der Weiterbildung, Leipzig.

PD Dr. Peter M Hejl

Privatdozent am Institut für Medienforschung der Universität Siegen, lehrt Soziologie mit Schwerpunkt Kommunikation und Medien. Projektleiter des Teilprojekts „Anthropologische und soziale Faktoren der Mediennutzung" am Forschungskolleg Medienumbrüche an der Universität Siegen. Forschungsschwerpunkte: systematische und anthropologische Grundlagen soziologischer Theoriebildung, insbesondere Wahrnehmungs- und Kommunikationstheorie aus konstruktivistischer Perspektive und ihre Zusammenführung zu einer Theorie sozialer Systeme. Zahlreiche Veröffentlichungen zur Kommunikations- und Medientheorie, zur soziologischen Systemtheorie, zur Geschichte der Soziologie und zur Beziehung Biologie/Soziologie.

\section{Prof. Dr. K. Ludwig Pfeiffer}

Professor für Anglistik und Allgemeine Literaturwissenschaft an der Universität Siegen. Zahlreiche Gastprofessuren und fellowships in den USA, Japan und Brasilien. Projektleiter (gem. mit Prof. Dr. Ralf Schnell) des Teilprojekts „Medienanthropologie und Medienavantgarde“ am Forschungskolleg Medienumbrüche an der Universität Siegen. Projektleiter des VW-Projekts „Mystik und Moderne“. Neuere größere Arbeiten: Das Mediale und das Imaginäre (1999), The Protoliterary (2002), Theorie als kulturelles Ereignis (Mithrsg., 2001). 
Prof. Dr. Dr. Gerhard Roth

Studium in Philosophie, Germanistik, Musikwissenschaften und Biologie. Promotion in Philosophie und in Zoologie. Professor für Verhaltensphysiologie und Entwicklungsneurobiologie an der Universität Bremen und Direktor am Institut für Hirnforschung. Rektor des Hanse-Wissenschaftskollegs in Delmenhorst. Forschungsgebiete: Kognitive und emotionale Neurobiologie sowie Neurophilosophie.

\section{Prof. Dr. Ralf Schnell}

Professor für Neuere deutsche Literaturwissenschaft und Medienwissenschaft an der Universität Siegen. Geschäftsführender Direktor des Instituts für Medienforschung der Universität Siegen und Sprecher des Kulturwissenschaftlichen Forschungskollegs Medienumbrüche sowie Mitherausgeber der Zeitschrift für Literaturwissenschaft und Linguistik und der Kölner Ausgabe der Werke Heinrich Bölls (27 Bde.). Schwerpunkte in Lehre und Forschung: Literarische Ironie; Heinrich Heine; Deutschsprachige Literatur seit 1945; Kunst, Literatur und Film im Dritten Reich; Medienästhetik; Filmtheorie. Ausgewählte neuere Buchpublikationen: Dichtung in finsteren Zeiten. Deutsche Literatur und Faschismus (1998); Medienästhetik. Zu Geschichte und Theorie audiovisueller Wahrnehmungsformen (2000); Orientierung Germanistik. Was sie kann, was sie will (2000); Lexikon Kultur der Gegenwart (Hrsg., 2000); Geschichte der deutschsprachigen Literatur seit 1945 (2003).

\section{Prof. Dr. Eckart Voland}

Studium der Biologie und Sozialwissenschaften an der Universität Göttingen. Promotion zum Dr. rer. nat. mit einer Arbeit zum Sozialverhalten von Primaten. DFG-Stipendiat. 1992 Habilitation (Uni Göttingen) für Anthropologie mit dem Thema „Historische Demographie und Soziobiologie“. Seit 1995 Professor für Philosophie der Biowissenschaften an der Universität Gießen. Forschungsarbeiten vorrangig auf den Gebieten der Evolutionären Anthropologie (Soziobiologie, Verhaltensökologie, Evolutionäre Ethik, Evolutionäre Ästhetik) und historische Demografie. 


\section{Die Titel dieser Reihe:}

Isabel Maurer Queipo,

Nanette Rißler-Pipka,

Volker Roloff (Hg.)

Die grausamen Spiele des "Minotaure"

Intermediale Analyse einer surrealistischen Zeitschrift

Mai 2005, ca. 250 Seiten,

kart., ca. $25,80 €$

ISBN: 3-89942-345-3

Walburga Hülk,

Gregor Schuhen,

Tanja Schwan (Hg.)

(Post-)Gender

Choreographien / Schnitte

April 2005, ca. 300 Seiten,

kart., ca. $27,00 €$,

ISBN: 3-89942-277-5

Ralf Schnell (Hg.)

Wahrnehmung - Kognition -

Ästhetik

Neurobiologie und

Medienwissenschaften

März 2005, 264 Seiten,

kart., $24,80 €$,

ISBN: 3-89942-347-X

Nicola Glaubitz,

Andreas Käuser,

Hyunseon Lee (Hg.)

Akira Kurosawa und

seine Zeit

März 2005, 314 Seiten,

kart., $27,80 €$,

ISBN: 3-89942-341-o
Isabel Maurer Queipo,

Nanette Rißler-Pipka (Hg.)

Spannungswechsel

Mediale Zäsuren zwischen den

Medienumbrüchen 1900/2000

März 2005, 220 Seiten,

kart., $23,80 €$,

ISBN: $3-89942-278-3$

Ralf Schnell,

Georg Stanitzek (Hg.)

Ephemeres

Mediale Innovationen

1900/2000

März 2005, 242 Seiten,

kart., $24,80 €$,

ISBN: 3-89942-346-1

Rainer Geißler,

Horst Pöttker (Hg.)

Massenmedien und die

Integration ethnischer

Minderheiten in Deutschland

Problemaufriss -

Forschungsstand -

Bibliographie

März 2005, 546 Seiten,

kart., 32,80 €,

ISBN: 3-89942-280-5

Leseproben und weitere Informationen finden Sie unter: www.transcript-verlag.de 


\section{Die Titel dieser Reihe:}

Marijana Erstic,

Gregor Schuhen,

Tanja Schwan (Hg.)

Avantgarde - Medien -

Performativität

Inszenierungs- und

Wahrnehmungsmuster $\mathrm{zu}$

Beginn des 20. Jahrhunderts

2004, 370 Seiten,

kart., $29,80 €$,

ISBN: 3-89942-182-5

Peter Gendolla,

Jörgen Schäfer (Hg.)

Wissensprozesse in der

Netzwerkgesellschaft

2004, 286 Seiten,

kart., $26,80 €$

ISBN: 3-89942-276-7

Michael Lommel,

Isabel Maurer Queipo,

Nanette Rißler-Pipka,

Volker Roloff (Hg.)

Französische Theaterfilme zwischen Surrealismus und Existentialismus

2004, 334 Seiten,

kart., $28,80 €$,

ISBN: 3-89942-279-1

Uta Felten, Volker Roloff (Hg.)

Spielformen der Inter-

medialität im spanischen

und lateinamerikanischen

\section{Surrealismus}

2004, 364 Seiten,

kart., $27,80 €$,

ISBN: 3-89942-184-1
Jens Schröter,

Alexander Böhnke (Hg.)

Analog/Digital - Opposition oder Kontinuum?

Zur Theorie und Geschichte einer Unterscheidung

2004, 438 Seiten,

kart., $32,80 €$,

ISBN: 3-89942-254-6

Matthias Uhl, Keval J. Kumar Indischer Film

Eine Einführung

2004, 174 Seiten,

kart., $18,80 €$,

ISBN: $3-89942-183-3$

Michael Lommel,

Isabel Maurer Queipo,

Nanette Rißler-Pipka (Hg.)

Theater und Schaulust im aktuellen Film

2004, 172 Seiten,

kart., 19,80 €,

ISBN: 3-89942-181-7

Leseproben und weitere Informationen finden Sie unter: www.transcript-verlag.de 